\title{
Power system damping controllers design using a backstepping control technique
}

\author{
Ali Karimi \\ West Virginia University
}

Follow this and additional works at: https://researchrepository.wvu.edu/etd

\section{Recommended Citation}

Karimi, Ali, "Power system damping controllers design using a backstepping control technique" (2008). Graduate Theses, Dissertations, and Problem Reports. 4388.

https://researchrepository.wvu.edu/etd/4388

This Dissertation is protected by copyright and/or related rights. It has been brought to you by the The Research Repository @ WVU with permission from the rights-holder(s). You are free to use this Dissertation in any way that is permitted by the copyright and related rights legislation that applies to your use. For other uses you must obtain permission from the rights-holder(s) directly, unless additional rights are indicated by a Creative Commons license in the record and/ or on the work itself. This Dissertation has been accepted for inclusion in WVU Graduate Theses, Dissertations, and Problem Reports collection by an authorized administrator of The Research Repository @ WVU.

For more information, please contact researchrepository@mail.wvu.edu. 


\title{
Power System Damping Controllers Design using a Backstepping Control Technique
}

\author{
by \\ Ali Karimi \\ Dissertation submitted to the \\ College of Engineering and Mineral Resources \\ at West Virginia University \\ in partial fulfillment of the requirements \\ for the degree of \\ Doctor of Philosophy \\ in \\ Electrical Engineering \\ Professor Muhammad A. Choudhry, Ph.D. \\ Professor Asadollah Davari, Ph.D. \\ Professor Sherman Riemenschneider, Ph.D. \\ Professor Matthew C. Valenti, Ph.D. \\ Professor Ali Feliachi, Ph.D., Chair \\ Lane Department of Computer Science and Electrical Engineering \\ Morgantown, West Virginia \\ 2008
}

Keywords: electric power systems, transient stability, backstepping control, particle swarm optimization, reinforcement learning

Copyright 2008 Ali Karimi 


\begin{abstract}
Power System Damping Controllers Design using a Backstepping Control Technique

by

Ali Karimi

Doctor of Philosophy in Electrical Engineering

West Virginia University

Professor Ali Feliachi, Ph.D., Chair
\end{abstract}

The objective of this dissertation is to design and coordinate controllers that will enhance transient stability of power systems subject to large disturbances. Two specific classes of controllers have been investigated, the first one is a type of supplementary signals added to the excitation systems of the generating units, and the second is a type of damping signal added to a device called a Static Var Compensator that can be placed at any node in the system. To address a wide range of operating conditions, a nonlinear control design technique, called backstepping control, is used. While these two types of controllers improve the dynamic performance significantly, a coordination of these controllers is even more promising. Control coordination is presented in two parts. First part concerns simultaneous optimization of selected control gains of exciter and SVC in coping with the complex nature of power systems. Second part proposes a combination of reinforcement learning and a backstepping control technique for excitation control system. The reinforcement learning progressively learns and adapts the backstepping control gains to handle a wide range of operating conditions. Results show that the proposed control technique provides better damping than conventional power system stabilizers and backstepping fixed gain controllers. 


\section{Acknowledgements}

I am deeply indebted to my supervisor and mentor Dr. Ali Feliachi for his advice, help

and support over the years. He has challenged, guided and urged me on with wisdom and patience. Without him, this project would not have materialized.

It is with such pleasure that I thank all the faculty members who have encouraged and supported me in unique ways. Special thanks go to Professor Muhammad Choudhry (WVU Department of Computer Science and Electrical Engineering), Professor Asadollah Davari (WVU Tech. Department of Electrical \& Computer Engineering), Professor Sherman Riemenschneider (WVU Department of Mathematics) and Professor Matthew C. Valenti (WVU Department of Computer Science and Electrical Engineering).

To my close friends and colleagues, Sara Eftekharnejad, Koroush Sedghisigarchi, and Karl Schoder whose faith in me remained boundless, my heartfelt thanks for every gift of personal kindness and scholarly encouragement.

And last, but not least, I dedicate this work with love and gratitude to my parents Kamran and Afarin Karimi, my amazing aunt, Noushin Nemazee, my uncle Reza Nemazee, and my cousins Shahin, Ali, Aidin, Carol, Alix and Asa. There are no words to express the depth of gratitude that underlies their love and support. They have all borne the burden of this work from beginning to end, and it is the quality and quantity of their love that has made this work possible.

This work was supported in part by grants from the US DEPSCoR/ONR grant No. N00014-03-1-0660, the US DoE grant No. DE-FC26-06NT42793, National Science Foundation grant No. ECS-9870041 and in part by US DoE EPSCoR WV State Implementation Award. 


\section{Contents}

$\begin{array}{ll}\text { Abstract } & \text { ii }\end{array}$

List of Figures $\quad$ vii

List of Tables $\quad$ xi

Notation and Acronyms xii

1 Introduction $\quad 1$

1.1 Problem Statement . . . . . . . . . . . . . . . . . . . . 1

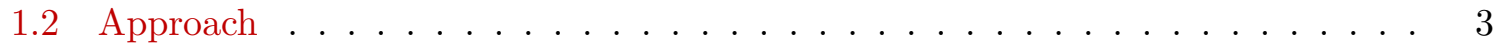

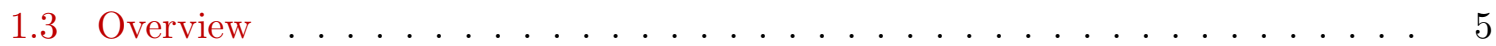

2 Literature Survey $\quad 7$

2.1 Introduction . . . . . . . . . . . . . . . . . . 7

2.2 Lyapunov Stability . . . . . . . . . . . . . . . . . . . . 7

2.3 Transient Stability . . . . . . . . . . . . . . . . . . . 11

2.4 Control Design Tools . . . . . . . . . . . . . . . . . . . 15

3 Background $\quad \mathbf{2 0}$

3.1 Backstepping Control Design for Simple Power System . . . . . . . . . . . . . 20

3.1 .1 Introduction . . . . . . . . . . . . . . . . 20

3.1.2 Backstepping Control Design . . . . . . . . . . . . . . . . 21

3.1.3 Power System Problem Formulation . . . . . . . . . . . . . . . 25

3.1 .4 Case Studies . . . . . . . . . . . . . . . . . . . 30

3.2 Function Optimization . . . . . . . . . . . . . . . . 34

3.2 .1 Introduction . . . . . . . . . . . . . . . . 34

3.2 .2 Optimization Problem . . . . . . . . . . . . . . 34

3.2.3 Particle Swarm Optimization Algorithm . . . . . . . . . . . . . 35

4 Transient Stability Enhancement Using Excitation Control 41

4.1 Power System Stability Enhancement using Backstepping Controller Tuned by

Particle Swarm Optimization Technique . . . . . . . . . . . . . . . 41

4.1 .1 Introduction . . . . . . . . . . . . . . . . 41

4.1 .2 Optimal Settings for Controller Gains . . . . . . . . . . . . . . 41

4.1 .3 Case Study . . . . . . . . . . . . . . . . . . . . . 43 
4.2 Extended-Backstepping Control of Power Systems . . . . . . . . . . . . . 48

4.2 .1 Introduction . . . . . . . . . . . . . . . . . 48

4.2.2 Backstepping Control Design in Presence of Disturbance . . . . . . . . . 48

4.2 .3 Disturbance Modeling . . . . . . . . . . . . . . . . . . . . . 52

4.2 .4 Overall System View . . . . . . . . . . . . . . . . . 53

4.2.5 Optimal Settings for Controller Gains . . . . . . . . . . . . . . 54

4.2 .6 Case Study . . . . . . . . . . . . . . . . . . . . . . 54

4.3 PSO-tuned Adaptive Backstepping Control . . . . . . . . . . . . . . . 63

4.3 .1 Introduction . . . . . . . . . . . . . . . . 63

4.3 .2 Problem Formulation . . . . . . . . . . . . . . . 63

4.3.3 Adaptive Backstepping Control Design . . . . . . . . . . . . . . . . 64

4.3.4 Optimal Settings for Controller Gains . . . . . . . . . . . . . . 68

4.3 .5 Case Study . . . . . . . . . . . . . . . . . . . 69

4.3 .6 Modeling Error . . . . . . . . . . . . . . . . . . . . . . 81

5 Transient Stability Enhancement Using SVC $\quad \mathbf{8 7}$

5.1 Static Var Compensator . . . . . . . . . . . . . . . . . . . 87

5.2 SVC Backstepping Damping Nonlinear Controller Design for Power Systems . . 89

5.2 .1 Introduction . . . . . . . . . . . . . . . . . . 89

5.2 .2 SVC Control Design . . . . . . . . . . . . . . . . . 89

5.2 .3 Optimal Settings of SVC Control Gains . . . . . . . . . . . . . . . . . 91

5.2 .4 Single Machine Infinite Bus . . . . . . . . . . . . . . . . . . 91

5.2 .5 Two Area System . . . . . . . . . . . . . . . . . . . 97

6 Control Coordination $\quad 106$

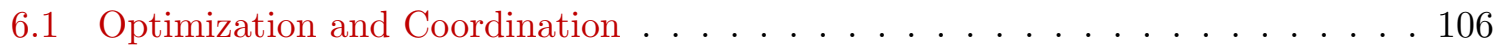

6.2 Problem Formulation . . . . . . . . . . . . . . . . . . 107

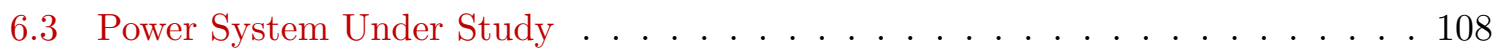

6.3.1 Excitation Control Design . . . . . . . . . . . . . . . 109

6.3 .2 SVC Control Design . . . . . . . . . . . . . . . . . 111

6.4 Optimum Settings for Controllers . . . . . . . . . . . . . . . . 112

6.5 Results and Discussion . . . . . . . . . . . . . . . . . . 113

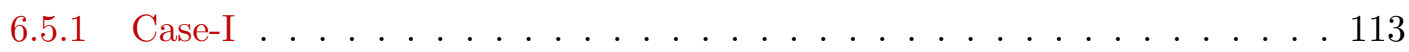

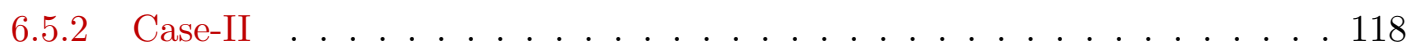

6.6 Advanced Control Technique . . . . . . . . . . . . . . . . 123

6.6.1 Reinforcement Learning . . . . . . . . . . . . . . . . 123

6.7 Proposed Control Design . . . . . . . . . . . . . . . . 126

6.8 Case Study . . . . . . . . . . . . . . . . . . . . . . . . . 129

7 Summary and Conclusions $\quad 137$

7.1 Completed Work . . . . . . . . . . . . . . . . . . 137

7.2 Suggestion for Future Work . . . . . . . . . . . . . . . . . 138

7.3 Accomplishments and list of publications . . . . . . . . . . 139

$\begin{array}{ll}\text { APPENDICES } & 142\end{array}$ 
A Single machine Infinite bus system data 143

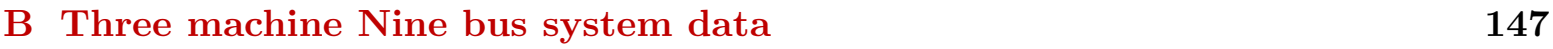

C $\quad$ Two Area-Four Machine system data 152

$\begin{array}{lr}\text { D Fifty Machine system data } & 157\end{array}$

E Matlab Code $\quad \mathbf{1 6 2}$

E.1 Particle Swarm Optimiztion . . . . . . . . . . . . . . . . . 162

E.2 Reinforcement Learning . . . . . . . . . . . . . . . . . 166

$\begin{array}{llr}\text { F Appendix-I } & 174\end{array}$

$\begin{array}{ll}\text { G Appendix-II } & 178\end{array}$ 


\section{List of Figures}

3.1 Backstepping Design Procedure . . . . . . . . . . . . . . . . . . 23

3.2 Single Machine Infinite Bus System . . . . . . . . . . . . . . . . . . 31

3.3 Relative rotor angle (deg), Generator's shaft speed (p.u.). Backstepping (solid line -), PSS (dash-dot line -.) and uncontrolled system (dotted line :) . . . . . 32

3.4 Terminal voltage at bus 3 (p.u.), and Control signal (p.u.). Backstepping (solid line -), PSS (dash-dot line -.) and uncontrolled system (dotted line :) . . . . . 32

3.5 Relative rotor angle (deg) and Generator's shaft speed (p.u.) . . . . . . . . . . 33

3.6 Terminal voltage at bus 3 (p.u.) and electric power deviation of generator (p.u.) 33

3.7 Particle movement toward new position . . . . . . . . . . . . . . 39

4.1 Three Machine Nine Bus Power System . . . . . . . . . . . . . . . . . . . 44

4.2 Excitation system control . . . . . . . . . . . . . . . . . 44

4.3 Relative rotor angles $2-1$ and $3-1$ (rad), exciter with backstepping damping controller with objective function $J(I)$ (solid line -), Objective function $J(I I)$ (dash line --), Objective function $J(I I I)$ (dash-dot line -.), PSS (dot line :)

4.4 Terminal voltages for generators 2 and 3, exciter with backstepping damping controller with objective function $J(I)$ (solid line -), Objective function $J(I I)$ (dash line --), Objective function $J(I I I)$ (dash-dot line -.), PSS (dot line :)

4.5 Control signals for generators 2 and 3, exciter with backstepping damping controller with objective function $J(I)$ (solid line -), Objective function $J(I I)$ (dash line --), Objective function $J(I I I)$ (dash-dot line -.), PSS (dot line :) 47

4.6 Backstepping Control and Nonlinear Damping . . . . . . . . . . . . . . . . 51

4.7 Fifty Machine System Single Line Diagram _ . . . . . . . . . . . . . . . . . 55

4.8 Supplementary Damping Controllers with Exciter . . . . . . . . . . . . . . 56

4.9 Relative rotor angles (rad) $6-1,6-3$. Exciter with PSO-tuned extendedbackstepping (solid line -), simple exciter with PSO-tuned PSS (dash-dot line -. ), simple exciter with GA-tuned PSS (dash line -- ), simple exciter without damping control (dot line .) . . . . . . . . . . . . . . . . .

4.10 Excitation Control signals for Generators 2 and 6. Exciter with PSO-tuned extended-backstepping (solid line -), simple exciter with PSO-tuned PSS (dashdot line -. ), simple exciter with GA-tuned PSS (dash line -- ), simple exciter without damping control (dot line .) . . . . . . . . . . . . . . . 58

4.11 Effect of nonliear damping speed deviation and control signal for generator 1, $\gamma=1500$ (solid line - ), $\gamma=100$ (dash-dot line .), $\gamma=15$ (dot line .) . . . . . 59 
4.12 Effect of nonlinear damping speed deviation and control signal for generator 5, $\gamma=1500$ (solid line -), $\gamma=100$ (dash-dot line .), $\gamma=15$ (dot line .) . . . . . 60

4.13 Relative rotor angles (rad) $6-1,2-6$. Simple exciter with extended backstepping control with $\gamma=1000$ (solid line - ), $\gamma=400$ (dash line -- ), $\gamma=90$

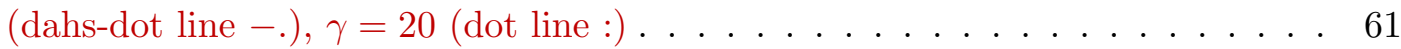

4.14 Adaptive backstepping Controller with Exciter . . . . . . . . . . . . . . 70

4.15 Relative rotor angle $\delta_{21}$ and control signal Generator 2. Adaptive Backstepping (solid line -), and Power System Stabilizer (dash-dot line -.), Exciter without supplementary signal (dot line . . . . . . . . . . . . . . . . .

4.16 Interface modeling for generators 2 and 6. Exact term $d^{\prime}$ (solid line -), esti-

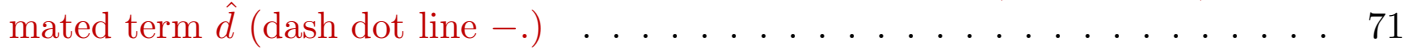

4.17 Relative rotor angle $\delta_{21}(\mathrm{deg})$ and Control signal generator 2. Adaptive Backstepping (solid line -), PSS (dot line :) . . . . . . . . . . . . 72

4.18 Interface modeling for generators 2 and 6. Exact term $d^{\prime}$ (solid line -), estimated term $\hat{d}$ (dash dot line.-$) \ldots \ldots \ldots$. . . . . . . . . . 73

4.19 Two area system . . . . . . . . . . . . . . . . . . . . 74

4.20 Adaptive backstepping Controller with static exciter . . . . . . . . . . . 75

4.21 Relative rotor angles $\delta_{31}, \delta_{41}$. Adaptive backstepping (solid line), PSS (dashdotted line) and uncontrolled system (dotted line) for Scenario-I . . . . . . . . 77

4.22 Relative rotor angles $\delta_{31}, \delta_{41}$. Adaptive backstepping (solid line), PSS (dashdotted line) and uncontrolled system (dotted line) for Scenario-II . . . . . . . . 77

4.23 Relative rotor angle $\delta_{31}$, Speed deviations $\Delta \omega_{3}$ for Generator 3. Adaptive Backstepping (solid line -), and PSS (dash-dot line -.) in Scenario-III . . . . . . 78

4.24 Terminal voltage at buses 11 and excitation field voltage $E_{f l d_{3}}$ for Generator 3. Adaptive Backstepping (solid line -), and PSS (dash-dot line -.) in Scenario-III 79

4.25 Interface modeling for generator 2 and 3. Exact term (solid line -) and estimated term (dash-dot line -.) in Scenario-III . . . . . . . . . . . . . . . 80

4.26 Speed deviation $\Delta \omega_{3}$ for several simulations, minimum (dash-dotted line), mean (solid line), and maximum (dotted line) values for $\Delta \omega_{3}$, Scenario-II . . . . . . . 82

4.27 Relative rotor angle $\Delta \delta_{31}$ for several simulations, minimum (dash-dotted line), mean (solid line), and maximum (dotted line) values for $\Delta \delta_{31}$, Scenario-II . . . 83

4.28 Relative angles $\Delta \delta_{21}, \Delta \delta_{31}$, and $\Delta \delta_{41}$. Electric power deviation output of Subtransient generator model (dotted line) and transient model (solid line) . . . .

4.29 Electric power deviations $\Delta P_{e 1}, \Delta P_{e 2}$, and $\Delta P_{e 3}$, and $\Delta P_{e 4}$. Relative angles of Subtransient generator model (dotted line) and transient model (solid line) . . 86

5.1 SVC Equivalent Model . . . . . . . . . . . . . . . . . . . . . . 88

5.2 SVC damping controllers with nonlinear control structure (backstepping) and Lead-Lags . . . . . . . . . . . . . . . . . . . . . . . . 990

5.3 SVC with lead-lag damping controller (dotted line :),SVC with backstepping damping controller (solid line -). Fault duration $40 \mathrm{msec}$. . . . . . . . . . . 94

5.4 SVC with lead-lag damping controller (dotted line :),SVC with backstepping damping controller (solid line -). Fault duration 66 msec . . . . . . . . . . . 95 
5.5 Terminal voltage under a large induction load and generator relative rotor angle $(\operatorname{deg}) \ldots \ldots \ldots \ldots \ldots \ldots \ldots \ldots$

5.6 Effect of low to high generation on electric power of generator and speed deviation of generator . . . . . . . . . . . . . . . . 96

5.7 Effect of low to high generation power on terminal voltage of generator . . . . . 97

5.8 Two Area system . . . . . . . . . . . . . . . . . . . . . . 97

5.9 Lead-Lag damping signal with electric power and speed inputs . . . . . . . . . 99

5.10 Relative angle $2-1$ and $4-1$ (deg) . . . . . . . . . . . . 100

5.11 Speed and terminal voltage for Generator $2 \ldots \ldots$. . . . . . . . . 102

5.12 Effect of time delay in relative angle $\delta_{21}$. Solid line (no delay), dash-dotted line

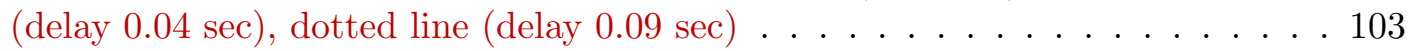

5.13 Effect of time delay in relative angles $\delta_{31}, \delta_{41}$. Solid line (no delay), dash-dotted

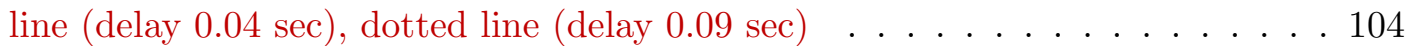

5.14 Relative rotor angles $\delta_{31}$ and $\delta_{41}$. Solid line (Excitation control with backstepping damping controller), dash-dotted line (SVC with backstepping damping controller $\ldots \ldots \ldots$. . . . . . . . . . . . . . . . . . 105

6.1 Four Machine System with Damping Controllers . . . . . . . . . . . . . . . . 109

6.2 Damping Controllers for Exciter and SVC . . . . . . . . . . . . . . . . 112

6.3 Relative rotor angles $\delta_{21}, \delta_{41}$ (deg). Optimized controller gains with $J_{1}$ (dash line), $J_{2}$ (dash-dot line), and $J_{3}$ (dotted line), $J_{4}$ (solid line) $\ldots \ldots \ldots$

6.4 Tie-lines power deviations between $3-101$ and $101-13$. Optimized controller gains with $J_{1}$ (dash line), $J_{2}$ (dash-dot line), $J_{3}$ (dotted line), $J_{4}$ (solid line) $\ldots 117$

6.5 Speed deviations Generators 1,4. Performance of controller with nominal gains (dotted line), effect of re-tuned control gains at $35 \%$ change of power generation $P_{\text {gen }}$ (solid line) . . . . . . . . . . . . . . . . . . . 119

6.6 Relative angle of generators $\delta_{21}, \delta_{41}$. Performance of controller with nominal gains (dotted line), effect of re-tuned control gains at 35\% change of power generation $P_{\text {gen }}$ (solid line) . . . . . . . . . . . . . . . . 119

6.7 Tie line 3-101 power deviations. Performance of controller with nominal gains (dotted line), effect of re-tuned control gains at $35 \%$ change of power generation $P_{\text {gen }}$ (solid line . . . . . . . . . . . . . . . . . 120

6.8 Speed deviations Generators 1, 4. Performance of controller with nominal gains (dotted line), effect of re-tuned control gains at $25 \%$ change of power generation $P_{\text {gen }}$ (solid line) . . . . . . . . . . . . . . . . . . 121

6.9 Relative angle of generators $\delta_{21}, \delta_{41}$. Performance of controller with nominal gains (dotted line), effect of re-tuned control gains at $25 \%$ change of power generation $P_{\text {gen }}$ (solid line) . . . . . . . . . . . . . . . 121

6.10 Schematic Design of Controlled System _ . . . . . . . . . . . . . . . . . 124

6.11 Block diagram representation of reinforcement learning . . . . . . . . . . . . . . 124

6.12 Schematic view of backstepping control architecture with reinforcement learner for two area power system . . . . . . . . . . . . . . . . . . 127

6.13 Scenario-1 Relative rotor angles $\delta_{31}, \delta_{41}$. Proposed control (solid line), Backstepping with fixed gains (dash-dotted line), PSS (dotted line) . . . . . . . 130 
6.14 Scenario-1 Control gains for generator $2,3 \ldots \ldots \ldots \ldots$. . . . . . . . 131

6.15 Scenario-1 Terminal voltages for Generators 1, $4 \ldots \ldots$. . . . . . . . . 132

6.16 Scenario-2 Relative rotor angles $\delta_{31}, \delta_{41}$. Proposed control (solid line), Backstepping with fixed gains (dash-dotted line), PSS (dotted line) . . . . . . . . 132

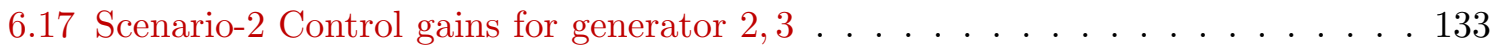

6.18 Scenario-2 Terminal voltage generator $2,4 \ldots \ldots \ldots$. . . . . . . . 134

6.19 Scenario-1 Supplementary control signals for generators $2,3 \ldots \ldots$

G.1 Schematic view of two generators with single transmission line in between . . . 178 


\section{List of Tables}

3.1 Synchronous generator and transmission line variables . . . . . . . . . . . 27

3.2 Backstepping Control Gains $k_{1}, k_{2} \ldots \ldots \ldots \ldots$

4.1 Parameters for Lead-Lag Stage $\left(T_{c}, T_{B}\right) \ldots \ldots \ldots \ldots$. . . . . . . . 44

4.2 Parameters for Backstepping Controller with Different Objective Functions . . 45

4.3 Parameters for PSS . . . . . . . . . . . . . . . . . . 45

4.4 Performance Index Parameters for Controller . . . . . . . . . . . . . . . 54

4.5 Parameters for Backstepping control . . . . . . . . . . . . . 57

4.6 Parameters for PSS tuned by PSO . . . . . . . . . . . . . . 57

4.7 Parameters for Backstepping control . . . . . . . . . . . . . . . . . 60

4.8 Adaptive Backstepping Controller Gains . . . . . . . . . . . . . . . . . . 70

4.9 PSS Gains . . . . . . . . . . . . . . . . . . . . . . . . . . . . . . . . . . . . . . .

4.10 Parameters for Static Excitation . . . . . . . . . . . . . . . . 74

4.11 PSS Gains . . . . . . . . . . . . . . . . . . . . 75

4.12 Adaptive Backstepping Controller Gains . . . . . . . . . . . . . . 76

4.13 Analyzed Scenarios . . . . . . . . . . . . . . . . 76

5.1 Analyzed Scenarios . . . . . . . . . . . . . . . . . . . . 92

5.2 Damping Controller Gains . . . . . . . . . . . . . . . . . . . . . . 94

5.3 Backstepping Controller Gains . . . . . . . . . . . . . . . 101

6.1 SVC and EXC Controller Gains . . . . . . . . . . . . . . . . . . . . . . 114

6.2 SVC and EXC Controller Gains . . . . . . . . . . . . . . . . . . . . . . . . . . . . . . . . . . . . . . .

6.3 Weighting factors for $J_{1}, J_{2}, J_{3}, J_{4} \ldots \ldots \ldots \ldots \ldots \ldots \ldots$

6.4 Exciter and SVC Controller Gains-25\% and $35 \%$ of active power generation and

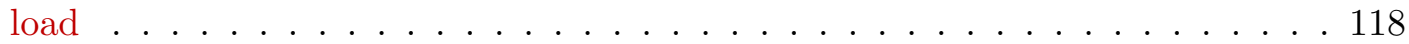

6.5 Parameters for Static Excitation . . . . . . . . . . . . . 129 


\section{Notation and Acronyms}

\section{Notation}

\section{Acronyms}

$\begin{aligned} \text { CIGRE } & \text { Conseil International des Grands Réseaux Électriques } \\ \text { CLF } & \text { Control Lyapunov Function } \\ \text { DFL } & \text { Direct Feedback Linearization } \\ \text { GA } & \text { Genetic Algorithm } \\ \text { IEEE } & \text { Institute of Electrical and Electronics Engineers } \\ \text { PSO } & \text { Particle Swarm Optimization } \\ \text { PSS } & \text { Power System Stabilizer } \\ \text { SMIB } & \text { Single Machine Infinite Bus } \\ \text { SVC } & \text { Static Var Compensator } \\ \text { EXC } & \text { Exciter } \\ \text { RL } & \text { Reinforcement Learning }\end{aligned}$




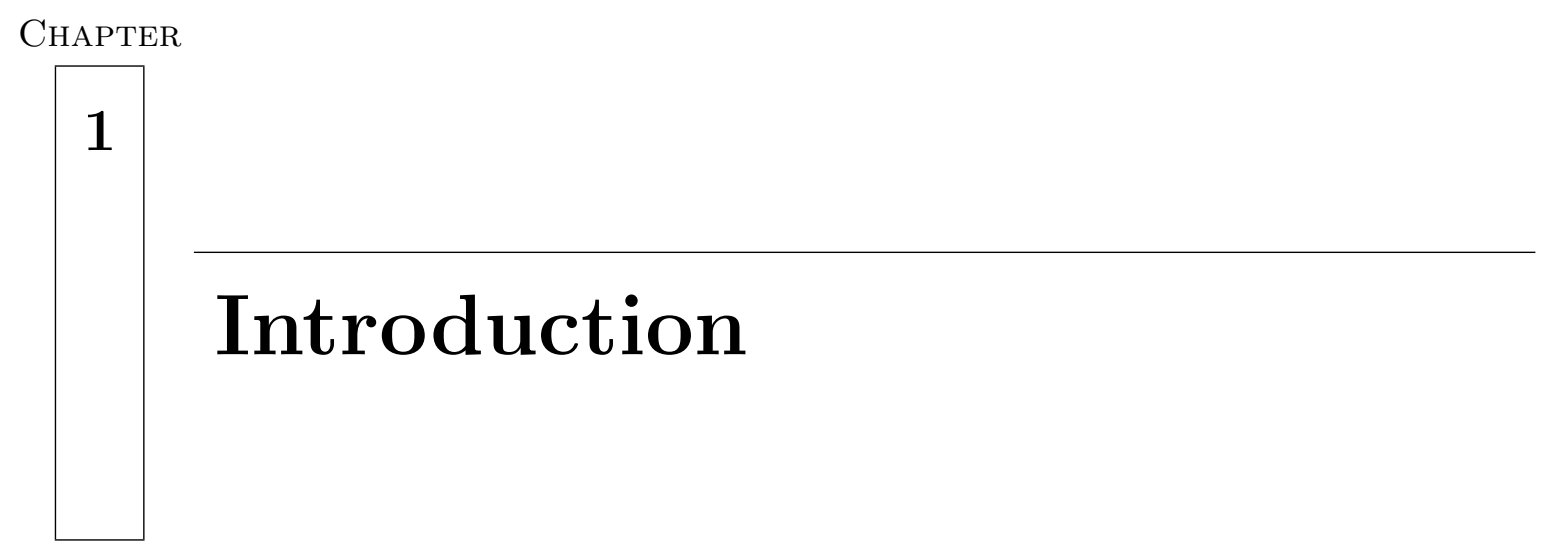

\section{$1.1 \quad$ Problem Statement}

The electric power system is a large complex interconnected system that constantly changes in structure due to load changes, disturbances, faults, installation of new devices and apparatus. Transients in the power system are analyzed using many levels of modeling details. Mathematical formulation is governed by differential algebraic equations that describe the dynamics of the generators in connection with the grid. These generators are nonlinear electromechanical systems that run synchronously. Following large system disturbances, some synchronous generators may swing enough to lose synchronism with the system or become transiently unstable.

Control is vital element to maintain the stability of the interconnected power system. Because of this need, control structures are becoming more pervasive and numerous, guaranteeing the stability of system over the wide range of operating conditions. They can be installed on generators, transmission lines, and distribution side. In this study, controllers are installed on generators and transmissions that can affect the stability of the overall grid.

With rapid growth of electric power system, the dynamic performance of controls, stability 
of systems, and methods of studying have changed correspondingly. Design and implementation of control techniques to enhance the stability of large scale power systems is a more challenging problem than ever before. The system itself is highly nonlinear, time varying, and geographically dispersed. With regard to above descriptions, the following questions are raised

- What is a feasible control structure to improve the stability of system?

- Is there a systematic way to obtain this control structure?

One of the most practical and economical ways of enhancing system stability is through excitation systems [66]. The excitation system can provides oscillation damping to power systems when augmented with supplementary controls. It can regulate the terminal voltage of generator when it is used with automatic voltage regulator. Other devices which can also play an increasing role in the operation and control of today's power systems are Flexible Alternating Current Transmission System or FACTS devices that certainly has influence on stability of power system [39]. FACTS devices are based on high-voltage and high-speed power electronics devices. There are capable of increasing the controllability of power flows and voltages enhancing the utilization and stability of existing systems.

Most available control techniques are based on linear power system models that are valid around the operating points. These include several designs for linearized models using a specific operating condition, making them prone to system changes. Power system by its nature is nonlinear, and it motivates to design a nonlinear control rather than linear one to avoid the need for linearization of power system equations. To enhance the safe operation of power system, the proposed controller has to perform satisfactorily over different scenarios that the system is likely to experience and dampen system oscillations without adversely affecting other aspects of system performance.

As for the system, electric power grid has been selected by the engineering academy as the first choice for the twentieth century engineering innovation that is most beneficial to civilization $[30,8]$. Therefore, a system with this level of attention and importance needs to be properly controlled as to guarantee stability following contingencies and disturbances. For such a complex electric power system, outage of single transmission line, generation unit or equipment failure might gradually lead into several more outages and eventually collapse of the entire system if it is not properly controlled. 
The dynamics of power system following considerable structural changes and loading conditions need to be controlled in order to ensure the system's integrity. These events can create dynamic stability problems in a fraction of a second [30]. The excitation control and FACTS devices are nowadays becoming a mean of equal importance in fast acting device to improve the stability of system. The progress made and aforementioned ideas motivated this dissertation with following approach.

\subsection{Approach}

Design of controller by implementing nonlinear control techniques has gained significant influence due to its inherent ability to improve control performance beyond what can be achieved by linear controls. In fact, linear analysis of complex nonlinear power system may fail in capturing some dynamic behaviors of the system especially in the events of critical faults or major disturbances. Today, nonlinear control theory forms a modern discipline that provides the tools necessary to improve dynamic performance so as to provide better quality and more secure power supply. Advances in computer systems and signal processing allow the practical implementation of these nonlinear controls.

The approach in this dissertation is as follows. On excitation control, first each generator is considered as a subsystem within the overall electric power system. Mathematical description of each subsystem is achieved by a set of algebraic differential equations. The equations include the interface among subsystems as well. Each subsystem, generator model, is transformed into strict feedback form [64]. This formation is suitable for implementing proposed control technique known as backstepping. Backstepping control is applied to each subsystem. The controller has gains that require proper tuning. Tuning of control gains should be coordinated with others and need to be done simultaneously so as to avoid adverse interactions. Hence, the control methodology is applied within the framework of a multi objective optimization problem to obtain optimum parameters for backstepping controls. The challenge lies in incorporating evolutionary computation with PSO to improve the performance of the proposed controller. By doing so, a tuned decentralized control scheme of system is achieved. Meaning that all the information for control at each subsystem are obtained and processed locally. Decentralized control has been a major research issue for many years by several researchers [91].

FACTS becomes a means equal importance to enhance stability of power system. SVCs 
are placed in the grid with the main purpose of the voltage support. These devices have several advantage including reduction of operation and transmission investment cost, increasing system security and reliability, increase power transfer capabilities, and overall enhancing a better quality of power and voltage. SVCs are shunt FACTS devices that can provide continuous and rapid control of reactive power and voltage, enhancing several aspects of transmission system performance, such as prevention of voltage collapse, transient stability enhancement during system oscillations. Generally, voltage regulation is the primary mode of operation, which improves voltage stability. However, the contribution of SVC to the damping of the system oscillations resulting due to the voltage regulation alone is not enough. Hence a damping controller is presented based on backstepping approach to enhance the damping of the system. The proposed controller is capable to provide additional damping during system oscillations for SVC. Centralized type of controller is designed with backstepping technique which requires remote information from each area. Conventional technique for designing these damping controllers are based on linear analysis based on one operating point which is not valid for wider range of operations. High degree of nonlinearity in power system and changes of operating conditions make the situation more challenging. Hence, nonlinear controller is designed based on backstepping control technique to achieve a good performance and enhance the stability under a wide range of operating conditions.

Damping of power system swings has important roles such as increasing the transmission capability, stabilizing the power system especially after large disturbance in the system. In power system several control devices are active simultaneously, hence it is important to ensure the stability of system in global and optimal manner. Simultaneous optimization of preselected parameters of exciter and SVC control parameters is performed based on particle swarm optimization (PSO) algorithm under transient conditions so that the stability of overall system can be improved over a wide range of operating conditions.

More advanced control technique is presented as an approach toward modern electric system. These new techniques lead to better, and in some cases guaranteed dynamic performance than conventional fixed parameter controllers. These control schemes have been based on both local measurements as well as measurements at different locations in the system resulting in decentralized and centralized approaches. In more specific, reinforcement learning algorithm [85] is applied to the backstepping controller on excitation system. These controllers are de- 
signed to withstand a set of credible contingencies in the system which satisfies their objective functions. Instead of having a pre-selected constant gains for controllers, the gains are optimized at first, then re-adjusted through reinforcement learning algorithm due to small and large disturbances which happens in the electric power grid.

\subsection{Overview}

The organization of this dissertation is as follows:

- Chapter 1: Introduction

The introduction consists of problem statement and approach. It continues with an overview and outline for this dissertation.

- Chapter 2: Literature survey

This chapter presents a survey concerning transient stability (section 2.3) and control design tools (section 6.19).

- Chapter 3: Background

This chapter includes basic background related to backstepping control design for simple power system. Also the function optimization with particle swarm is explained.

- Backstepping control design and implementation on simple power system (section 3.1)

- Function Optimization (section 3.2)

- Chapter 4: Transient Stability Enhancement Using Excitation Control

This chapter includes proposed controller schemes for electric power systems:

- Backstepping control tuned by particle swarm optimization technique (section 4.1)

- Extended-backstepping control in presence of disturbance (section 4.2)

- Adaptive backstepping control in presence of uncertainties (section 4.3)

- Chapter 5: Transient Stability Enhancement using SVC

- Static Var Compensators (section 5.1) 
- Application of backstepping control in Static Var Compensator (section 5.2)

\section{- Chapter 6: Control Coordination}

This chapter includes two parts.

- On first part, a simultaneous tuning procedure for nonlinear damping controllers for SVC and generator exciter is presented, using particle swarm optimization (6.1).

- On second part, advanced control technique is presented. Reinforcement learning scheme is presented and improves the performance of the controller for generator exciter in a multi machine power system (6.6).

- Chapter 7: Summary and conclusions

- Completed Work (section 7.1)

- Suggestion for Future Work (section 7.2)

- Accomplishments and list of publications (section 7.3)

This chapter summarizes the benefits of the developed nonlinear control and the enhancements achieved by designing nonlinear damping controls using the proposed technique, gives suggestions for future work, and lists research work already done or in progress that is based on parts of this dissertation.

- Appendices A-D include system benchmark data and Appendix E include MAT$\mathrm{LAB}^{1}$ computer codes. Finally, a lemma and mathematical simplification related to interconnection term are covered in Appendices F and G respectively.

All Case studies presented, are performed with the help of the Power Analysis Toolbox (PAT), a simulation package developed by Advanced Power and Electricity Research Center (APERC) at West Virginia University [82]. PAT includes advanced vectorized computations as well as a block-oriented simulation in MATLAB environment. The conditions for performing steady-state analysis, including load flow calculations and voltage-stability analysis are included. In addition, it has the capability of transient stability.

\footnotetext{
${ }^{1}$ MATLAB and Simulink are products of The Mathworks, Inc., http://www.mathworks.com/
} 


\section{Chapter}

2

\section{Literature Survey}

\section{$2.1 \quad$ Introduction}

In this chapter a literature survey related to the problem investigated in this dissertation is presented. The survey is organized as follow. In section 2.2 work related to Lyapunov stability is presented. Section 2.3 presents transient stability and related works. In Section 6.19 a survey on control design tools is given.

\subsection{Lyapunov Stability}

In this section the tools of Lyapunov stability theory is reviewed. These tools will be used in the following chapters to analyze the stability properties of proposed nonlinear controller. Consider the dynamic system:

$$
\dot{x}_{i}=f(x, t), \quad x\left(t_{0}\right)=x_{0} \quad x \in R^{n}
$$

It is assume that $f(x, t)$ satisfies the conditions for the existence and uniqueness of solution, $x$ and $t$ denotes states and time respectively. A point $x^{*} \in R^{n}$ is an equilibrium point of (2.1) if $f\left(x^{*}, t\right) \equiv 0$. Equilibrium point is locally stable if all solutions which start near $x^{*}$ remain near $x^{*}$ for all time. The equilibrium point $x^{*}$ is said to be locally asymptotically stable if $x^{*}$ 
is locally stable and all solutions starting near $x^{*}$ tend toward $x^{*}$ as $t \rightarrow \infty$. By shifting the origin of the system, it is assumed that the equilibrium point of interest occurs at $x^{*}$. In case of multiple equilibrium point, each need to to be studied by shifting the origin appropriately. Several definitions are described in following:

- Stability in the sense of Lyapunov: The equilibrium point $x^{*}=0$ of (2.1) is stable in sense of Lyapunov at $t=t_{0}$ if for any $\epsilon>0$ there exists a $\delta\left(t_{0}, \epsilon\right)>0$ such

$$
\left\|x\left(t_{0}\right)\right\|<\delta \Rightarrow\|x(t)\|<\epsilon, \quad \forall t \geq t_{0}
$$

Lyapunov stability does not require that trajectories starting close to the origin tend to the origin asymptotically. Also stability is defined at $t=t_{0}$. Uniform stability is a concept which guarantees that the equilibrium point is not losing stability. For the uniformly stable equilibrium point $x^{*}$, the upper bound $\delta$ is not a function of $t_{0}$, so the equation (2.2) may hold for all $t_{0}$.

- Asymptotic Stability: An equilibrium point of 2.1 is asymptotically stable at $t=t_{0}$ if $x^{*}=0$ is stable, and locally attractive. Meaning that

$$
\left\|x\left(t_{0}\right)\right\|<\delta \Rightarrow \lim _{t \rightarrow \infty} x(t)=0
$$

As in the previous definition, asymptotic stability is defined at $t_{0}$. Uniform asymptotic stability requires that $x^{*}=0$ is uniformly stable, and $x^{*}=0$ is uniformly locally attractive.

An equilibrium point is called unstable if it is not stable. Note that the aforementioned definitions are local definitions which describe the behavior of a system near equilibrium point. By definition equilibrium point $x^{*}$ is globally stable for all initial conditions $x_{0} \in R^{n}$. Global stability is an ideal and desirable, but in application of large scale nonlinear systems such as electric power system can be difficult to achieve.

Lyapunov's first method or indirect method of Lyapunov provides a linearization of system to determine the local stability of the original system. Given the system

$$
\dot{x}=f(x, t)
$$

with $f(0, t)=0$ for all $t \geq 0$. Define $J(t)$

$$
J(t)=\left.\frac{\partial f(x, t)}{\partial x}\right|_{x=0}
$$


to be the Jacobian matrix of $f(x, t)$ with respect to $x$, and evaluated at the origin. The system

$$
\dot{x}=J(t) x
$$

is defined as the linearization of equation (2.1). When the linearization exists, its stability determines the local stability of the original nonlinear system. From the linearized model, when Jacobian $J$ has all eigenvalues with negative real parts, the linearized equation (2.6) is called asymptotically stable. For this case initial conditions of states $x$ lying in a finite region around the origin, where the initial displacement of the state $x$ from the origin within this origin will decay to zero as time goes to infinity (asymptotically stable). This type of analysis is called "stability in the small" [65]. On the other hand "stability in the large" may be studied by explicit solution of the nonlinear differential equations.

Second or direct method of Lyapunov has proved subsequently to be more important than the first method since it directly related to nonlinear system and allows determining the stability of a system without explicitly integrating the differential equations. The approach based on the direct method would consist of characterizing the dynamic behavior of the system using suitable Lyapunov function. The idea is that if there is some measure of energy in the system, then the rate of change of that energy to ascertain stability can be studied. For better clarification of this concept, let $B_{\epsilon}$ be a ball of size $\epsilon$ around the origin where

$$
B_{\epsilon}=\left\{x \in R^{n}:\|x\|<\epsilon\right\}
$$

- Locally Positive Definite Function: A continuous function $V: R^{n} \times R_{+} \rightarrow R$ is a locally positive definite function if for some $\epsilon>0$ and some continuous, and strictly increasing function $\alpha: R_{+} \rightarrow R$.

$$
V(0, t)=0, \text { and } V(x, t) \geq \alpha(\|x\|) \quad \forall x \in B_{\epsilon}, \forall t \geq 0
$$

- Positive definite functions: A continuous function $V: R^{n} \times R_{+} \rightarrow R$ is a positive definite function if it satisfies the conditions of locally positive definite function, additionally $\alpha(p) \rightarrow \infty$ as $(p) \rightarrow \infty$.

- Decrescent functions: A continuous function $V: R^{n} \times R_{+} \rightarrow R$ is a decrescent function, if for some $\epsilon>0$ and some continuous strictly increasing function $\beta: R_{+} \rightarrow R$

$$
V(0, t)=0, \text { and } V(x, t) \leq \beta(\|x\|) \quad \forall x \in B_{\epsilon}, \forall t \geq 0
$$


In brief the direct method states that depends on $V(x, t)$ and $\dot{V}(x, t) \leq 0$ then it is possible to specify the stability condition of system. The time derivative of $V$ is taken along the trajectories of the system:

$$
\left.\dot{V}\right|_{\dot{x}=f(x, t)}=\frac{\partial V}{\partial t}+\frac{\partial V}{\partial x} f
$$

Let $V(x, t)$ be a non-negative function with derivative $\dot{V}$ along the trajectories of the system. Stability conditions are summarized a follow:

- If $V(x, t)$ is locally positive definite and $\dot{V}(x, t) \leq 0$ locally in $x$ and for all $t$, then the origin of the system is locally stable in sense of Lyapunov.

- If $V(x, t)$ is locally positive definite and decrescent, and $\dot{V}(x, t) \leq 0$ locally in $x$ and for all $t$, then the origin of the system is uniformly locally stable in sense of Lyapunov.

- If $V(x, t)$ is locally positive definite and decrescent, and $-\dot{V}(x, t)$ is locally positive definite, then the origin is uniformly locally asymptotically stable.

- If $V(x, t)$ is positive definite and decrescent, and $-\dot{V}(x, t)$ is positive definite, then the origin of the system is globally uniformly asymptotically stable.

In general, the second method of Lyapunov gives sufficient conditions for the stability of a origin of the system [75]. It does not provide information on how to provide the Lyapunov function $V(x, t)$, hence the search for a Lyapunov function establishing stability of an equilibrium point could be subtle. For the electric power grid it is usually hard to find the Lyapunov function which is also not a unique function. Furthermore, while the condition of Lyapunov stability theorem is sufficient, this does not specify on how to obtain the Lyapunov functions. A natural choice for the Lyapunov function is the system energy. A detailed analysis of the kinetic and potential energy behavior along time domain trajectory was conducted by several researchers and is addressed in literature [81], [31], [70]. In their analysis the Lyapunov function is composed from the kinetic energy associated with gross motion of angular speed of generators plus the potential energy which relates to inter-machine motion between the generators. 


\subsection{Transient Stability}

The electric power systems have grown in size and complexity with large number of interconnections. The design of such a system and its secure operation is still a challenging operation. To maintain a reliable service, a bulk electric grid must remain intact and be capable of withstanding a variety of small and large disturbances. Stability has been a major concern in electric power system for several years. In evaluation of stability the concern is the behavior of the power system when subjected to a transient disturbance, which may be small or large. The system is stable if it is able to operate satisfactorily under these conditions and effectively supply the maximum demand. Having provided the definitions of stability, the formation of stability procedure is presented in following. The system is initially at pre-disturbance steady state conditions presented by equation

$$
\dot{x}(t)=f^{p}(x(t)) \quad-\infty \leq t<0
$$

Superscript indicates the pre-disturbance situation. The system is at equilibrium, and the initial conditions are obtained by solving the power flow. Meaning that for a given power grid, with known complex power loads and generations with their constraints, solve for any unknown bus voltages and unspecified power generations and finally for the complex power flow in the grid components. Now consider at time $t=0$ the fault is initiated in system. As a result of fault occurrence, the dynamic of the system changes as:

$$
\dot{x}(t)=f^{f}(x(t)) \quad 0<t \leq t_{c l}
$$

where the superscript $f$ indicates the fault conditions. The fault is cleared by implementing protective devices at time $t_{c l}$. As a result the dynamic of the system is changed to postdisturbance which is formulated as

$$
\dot{x}(t)=f(x(t)) \quad t_{c l}<t<\infty
$$

the stability analysis is performed with objective to achieve the asymptotic stability of equilibrium point of system.

In reality the power system is a large complex system which is interconnected. As the complexity of system grows, the challenge to its secure operation grows as well [29]. From stability point of view, designing controllers for large interconnected system to ensure stable operation 
is a challenging problem. Power system is highly related to synchronous generators for generation of electric power. The necessary condition for transmission and exchange of power is that all generators rotates at synchronism.

In evaluation of stability, the major concern is the ability of electric power system to remain inact and capable of withstanding a variety of disturbances. Based on IEEE/CIGR Joint Task Force on Stability Terms and Definitions [41], Power System Stability is defined as follow:

"The ability of an electric power system, for a given operating condition, to regain a state of operating equilibrium after being subjected to a physical disturbance, with most system variables bounded so that particularly the entire system remains inact"

Disturbances might be small or large. For instance continuous load changes are considered as small disturbances but loss of long transmission line or trip of large generator from the grid are considered as large disturbances. In this study mostly the later type of disturbance is in concern. Hence, controllers are designed in a way to withstand large disturbances in the system. Based on [41], classical term concerning stability related to these type of disturbances is called transient stability and is defined as

"Ability of power system to maintain synchronism when subjected to severe disturbances"

The instability of power system can take different forms and can be affected by wide range of factors. Hence, it is important to identify the factors that contribute to unstability, and to form the method of improving stable operations. The classification of stability will greatly simplify this purpose. The main concern in this study is focused on transient stability. The time frame concern in transient stability studies, is almost $3-5$ seconds following a large disturbance. Also for very large systems the time is extended to 10-20 seconds [41].

When disturbance happens in the system, it upsets the balance between prime mover mechanical power and generator electrical power that some generators, possibly close to disturbance, attain deviation from synchronous speed. And when one generator rotates faster than the other, angular position of its rotor relative to the slower one will increase as well. 
This separation of angular position of rotor angles might increase further if the system can not absorb enough kinetic energy corresponding to this deviation of speed. As a result it leads to loss of synchronism and unstability. The concept is judged by variation of rotor angles of generators with respect to time. If a rotor angle of a generator or group of generators continue to increase with respect to the rest of the generators, the system is considered as unstable. It is common to measure rotor angle of generator with respect to a fixed reference frame known as synchronous rotating reference frame. Hence the synchronism is lost once rotor angle of generator or group of generators are separated away from the rest of generators.

The prevention of electric power system losing synchronism after a large disturbance is of great importance. Stability study of power system oscillations shows that power systems contain many modes of oscillations due to interactions between different components. Many of the oscillations are due to synchronous generator relative rotor swings. These oscillations often grows in magnitude over the span of seconds. In some cases they may cause larger generator groups to lose synchronism where part or overall of electric system is lost. Sustained oscillations may disrupt the power system even if they do not cause electric grid separation and loss of generations. For instance power swings may have associated voltage and frequency deviations that are unacceptable. One practical way to enhance the stability of synchronous generator is through excitation system equipped with supplementary control [65]. Excitation system contributes to effective control of voltage and ensure stability for large disturbance. Basically it provides DC voltage to field windings of generator and modulates this voltage for control objectives. Several configuration of excitation systems are included in IEEE standard actuators [42].

Supplementary control is frequently included in error voltage junction to actuators. It provides additional input signal to actuator (either exciter or FACTS devices) to damp power system oscillations. The early concepts of supplementary control were established in [23]. A common supplementary control is known as Power System Stabilizer (PSS). Carefully tuned PSS on the major generator units can damp the power oscillations. PSS consists of wash-out stage and lead-lag stage. Wash-out stage has stabilizer gain and wash-out time constant. Wash-out stage is a high pass filter to eliminate the input's steady state. Time constant is usually within the range of $1-20 \mathrm{sec}$, and let the stabilizing signal pass within the frequency of interest. Two first order phase compensation are usually used to produce component of electric torque in 
phase with speed deviation. Shaft speed, integral of electric power, and terminal frequency are the most common input to this control [65]. These inputs are measurable at each generator so controller can utilize local information and operate without the need for remote signals from other generators. Two inputs PSS has also been implemented for small and large signal stability. Analysis was performed on several test systems and on an actual Hydro-Quebéc system [48].

PSS has fixed parameters, lead-lag and wash-out stages, to be tuned. Tuning is off-line which is based on finding set of parameters in which PSS can damp the oscillations. Tuning procedure for PSS gained a lot of attention among researchers and large body of early works related to this matter [96]. But most of these techniques are based on eigen analysis where first state space models are obtained and then computation is performed based on these linearized models [12].

The controller design for linear system is achieved to give specific performance at one operating point which could be chosen as a worse case. There are sometimes intuitive ways to overcome these limitations to some extent. For instance in damping oscillations with PSS a range of oscillation frequencies can be dealt with by improved bandwidth design. Chow et. al [20] have used frequency domain robust control techniques to design new PSS structures. Their approach is based on unstructured uncertainty model which uses optimization over a class of stabilizing controllers to force a closed loop transfer function to be as close as possible to the desired one. Despite promising responses, robust control makes no restriction on how the parameters vary in the specified ranges. Since, in principle they could be jumping randomly and the controllers tend to be a high gain, and high bandwidth type which results in high cost of control. Besides robust control, linear adaptive controllers have also been presented [21]. For slow or infrequently changes of parameters, adaptive linear control can be implemented to automatically retune the controller. Severe problem with linear adaptive control is transient performance. Without good initial estimate of the parameters, the transient may be unacceptable in practice.

In recent years, more sophisticated tuning techniques based on evolutionary algorithms are developed for tuning control parameters. These techniques have the advantage of simultaneous tuning of control parameters through the frame work of multi objective optimization problem. Some innovative works related to PSS tuned by means of genetic algorithms has been done 
by do Bomfim, Taranto and Falcão [25]. Abido and Abdel-Magid presented tabu search (TS) optimization technique for optimal gains of conventional PSS [5]. Also Abido investigated the application of particle swarm optimization (PSO) for optimal settings of PSS gains through eigenvalue based objective function and the nonlinear simulation of multi machine power system $[3]$.

So far the control schemes that have been discussed only deal with small disturbances about an operating point. For large disturbance, there are pre-fault, fault-on, and post-fault operating points which might be very different. Despite promising results obtained through the course of their research, PSS still may not work properly during unpredictable changes. Beside sophisticated tuning technique, the control structure itself is of great importance. Hence, there is growing justification for nonlinear control structure which is capable to work effectively under different circumstances. Unlike PSS, the structure of this nonlinear control neither includes a phase compensation nor washout stage.

Through the course of this research, main concerns are

- Construct nonlinear control as supplementary control

- Obtain procedure for tuning the control parameters in optimal way

- Implement proposed controller on multi machine power systems

- Enhances the stability of system under different operating conditions

- Compare the proposed control performance with existing supplementary controller (PSS)

\subsection{Control Design Tools}

This section relates to survey on nonlinear control design and its application in power system. The innovative and early works on nonlinear control for power system are reviewed as follow.

Starting with the control tools implemented on simple single-machine-infinite-bus (SMIB) systems, Bazanella, Kokotovic̀ and e Silva [11] designed state feedback control based on a Lyapounov approach known as $L_{g} V$, where Lyapunov derivative depends on a control and can be made negative by feedback to improve dynamic performance of the system. This control methodology originated by Sontag [84]. Controller was implemented on simplified single-axis generator model. States of the generator are considered as rotor angle, shaft speed deviation, and internal voltage of generator. The controller, based on $L_{g} V$ requires the internal voltage 
of generator, which is not measurable. In their study, it is assumed that this internal variable is available.

An optimal excitation control strategy using Lyapunov direct method is proposed by Machowski, Bialek, Robak, and Bumby [70] and implemented on simple SMIB. Traditional Lyapunov direct method has been used in power system to evaluate the stability margin [81]. But here the Lyapunov direct method is used to obtain control strategy. Lyapunov function is constructed from kinetic and potential energy of system plus two additional terms to account for flux decrement effects on generator axis. The control structure has linear relationship between field voltage and internal voltage of generator is considered. Assumptions such as availability of generator internal voltage, availability of initial values of quadrature and direct voltage are considered during this study.

A so called Direct feedback linearization, based on the differential geometry approach [43], has been investigated by several researchers [89], [44], [45]. They applied DFL controller method to generator dynamics. The technique is capable of transforming system into linearized model by implementing compensation laws to cancel nonlinearities of system. This cancellation requires some unmeasurable variables. Also it requires large control effort to cancel complete nonlinearities of system. Although it is not always helpful to cancel all the nonlinearities in the system. The obtained linearized model is not an accurate model in comparison to the exact one. In their design, the main concerns are to prevent an electric power system from losing synchronism after a large sudden fault on system and achieving the good post fault regulation of generator terminal voltage.

Application of former control technique is extended to multi machine power system [35], [44]. In order to achieve decentralization of control, interconnection bounds between generator and the rest of the system is considered as a polynomial function of absolute rotor angle and speed deviation. Through obtaining mathematical bounds for interconnection terms, several assumptions are considered. For instance simple linear relation between excitation field voltage and internal voltage of generators. The proposed technique is tested on two machine infinite bus with objective to maintain stability of the system when large fault occurs.

In multi machine power systems, the same technique has been combined with observation decoupled state space (ODSS), originally introduced in [92], to achieve decentralization of control. Feedback linearization is used to handle nonlinearities and ODSS technique is used 
to achieve decoupling of each generator [28].

Different adaptive version of feedback linearization control were presented by Jain et al. [46]. In their analysis, equivalent reactances of the transmission lines were considered as unknown or varying parameters, then adaptation is used to estimate them and achieve an exact cancellation of terms by feedback linearization. The technique was implemented on a two generator infinite bus system. Their approach has been extended to a class of nonlinear systems with decentralized output feedback control, where the interconnection terms were expressed by polynomials [47].

Edwards and Spurgeon proposed a methodology based on a sliding mode control strategy to stabilize multimachine power system by using static output feedback [90]. Interconnection bounds are modeled similar to the approach as mentioned in [35] and the control is implemented on two machine infinite bus. The main idea of sliding mode control was established by Utkin [86]. Despite good robust characteristic, this control usually has steady state chattering effect which is not ideal for implementation on actual hardware in excitation control of generator.

Okou, Akhrif, and Dessaint presented a hierarchical control structure based on wide area signals using input-output linearization and parameters adaptation. However, both local and remote signals are assumed to be available. Local controllers dampen local rotor shaft oscillations and a centralized controller decouples subsystems interactions. Final control is obtained with a two-level objective of voltage and rotor speed regulator. Wide area control laws are derived from a reformulation of the multi-machine model. Terminal voltages of generators are considered as state variables instead of internal field voltages. As a result, the new model is obtained through complex mathematical transformations. The hierarchical controller is implemented on this model $[72,73]$.

More sophisticated and yet realistic control design technique, known as backstepping, has been developed by Krstić, Kanellakopoulos, and Kokotović [32, 49, 50, 64]. A complete review on the potential of this control including robust backstepping, adaptive backstepping, and observer-based backstepping is presented in 1991 Bode Lecture by Kokotović [62].

The first application of backstepping control in power system is given in [79] for SMIB system with the goal of improving both transient stability and voltage regulation. One-axis 
generator is considered for their study. Rotor angle, speed deviation and internal voltage of generator are considered as system states. Although the control gains are obtained through trial and error (without any optimization procedure), the results demonstrated the effectiveness of this novel control technique.

Discrete adaptive backstepping has also been implemented on single machine infinite bus [93]. Reactances and transient time constants of generators are considered as uncertainties. Backstepping excitation control is used to enhance transient stability of system with assumption of full availability of generator states. Meanwhile, adaptation laws are used to estimate the generator parameters.

With advances in power electronic devices, efficient damping can be achieved through the use of network devices such as static var compensators. The main purpose of SVC is the dynamic support of voltage. Once installed in a system, SVCs can also provide damping to provide damping to enhance transient stability using an auxiliary signal over SVCs voltage control loops. Several damping controllers have been designed and implemented for SVC. The majorities of which are composed of lead-lag phase compensation and filter blocks with adjustable parameters [65]. These designs are based on linearized model that is valid over a limited range of conditions. However, linear methods may not properly capture the dynamics of nonlinear power systems. Results in [18] indicate that a lead-lag damping controller designed may lead to system instability by changing the nature of the load. In terms of control input several lead-lag damping controllers have been designed using local measurements [94],[76]. Remote signals, such as generator speed or speed difference between two area have also been used as inputs to lead-lag structures [37],[22]. Nonlinear control techniques have also been implemented. A bang-bang controller combined with a linear one has been presented in [69]. This controller uses a phase angle signal that is obtained from the bus voltage and active power at the location of the SVC. Optimization of this control is mathematically complex and the nonlinearity of signal may introduce harmonics in voltages and currents that affect the control signal. Direct Feedback Linearization is also used as nonlinear damping controller for SVC which is mainly used for voltage stability problem [87].

The Reinforcement Learning (RL) is emerging as an important alternative to complex control problems. Sutton and Barto have presented the convergence of the algorithm and their applications in nonlinear system [85]. In domain of power system with application for 
damping oscillations, algorithm is proposed by Glavić et. al [33], [34]. Their idea is to combine Control Lyapunov Function (CLF) with RL in attempt to optimize a mix of system stability and performance. The capability of the proposed control is demonstrated on a control problem involving Thyristor Controlled Series Capacitor (TCSC) and dynamic brake controller for damping oscillations in power system [26]. In principle, based on their approach any control with stability guarantees can be combined with RL mode and any heuristic search technique can be used with Lyapunov based controller.

Coordination between controllers plays an important role once the controllers are installed and active at the same time. Following a disturbance in a system, due to uncoordinated control strategy used in many power systems, destabilizing interactions among SVC and exciter controllers is possible. The interaction of rotor dynamics under weakly interconnected system condition can cause dynamic stability and limit the operation range for power generators. Some approaches based on linearization of system are addressed for coordination among SVC and exciter controllers [77], [80]. However linear method can not properly capture complex dynamics of the system, especially during large disturbance. More advanced optimization and coordination techniques between FACTS devices and PSSs based on quasi-Newton algorithm have been covered in [68], [14].

The trends of power system and the modern control techniques are reviewed in this section. The survey looked at some modern control tools that have been offered in power system context for the past several years. In particular nonlinear control and their applicability to various classes of power system control problem is considered. 


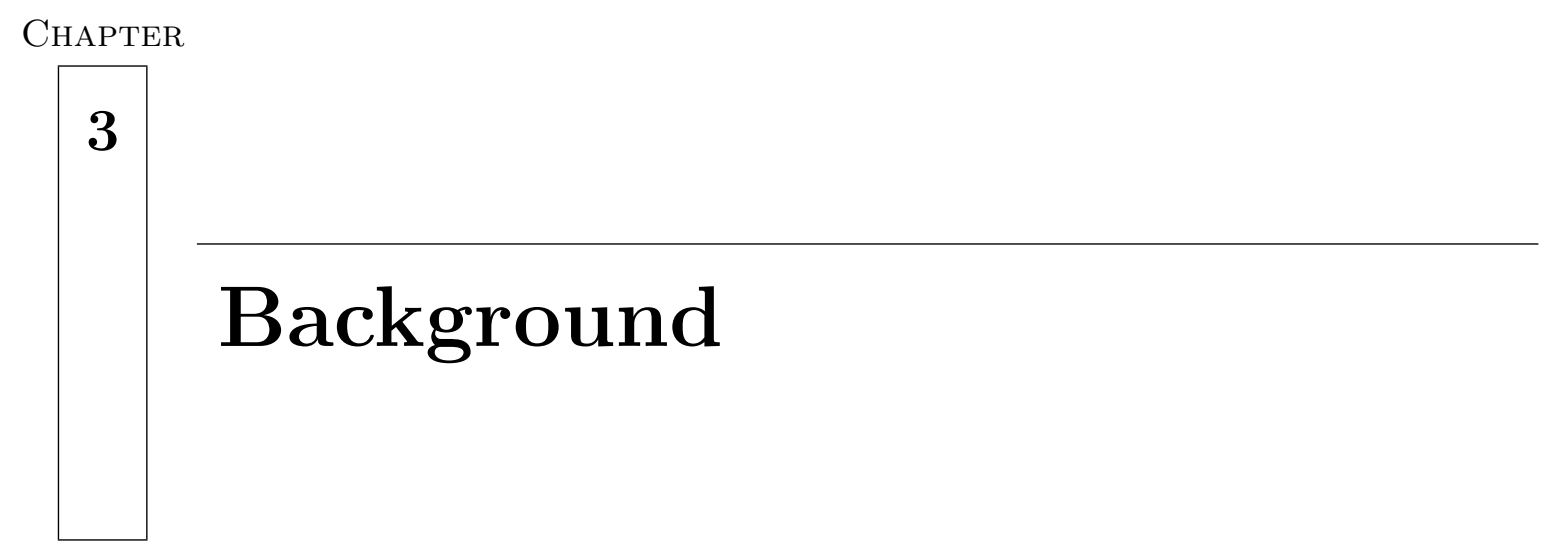

\subsection{Backstepping Control Design for Simple Power System}

\subsubsection{Introduction}

Organization of this section is as follows. Subsection 3.1.2 explains backstepping control technique applied to strict feedback system. In Subsection 3.1.3 power system model is brought into strict feedback form and backstepping control is applied. Case study with single machine infinite bus is given in Subsections 3.1.4. 


\subsubsection{Backstepping Control Design}

Backstepping procedure is described in this section for a specific form of system model known as strict feedback form [64]:

$$
\begin{aligned}
\dot{x} & =f_{0}+g_{0} z_{1} \\
\dot{z}_{1} & =f_{1}+g_{1} z_{2} \\
\dot{z_{2}} & =f_{2}+g_{2} z_{3} \\
\vdots & \\
\dot{z}_{k-1} & =f_{k-1}+g_{k-1} z_{k} \\
\dot{z}_{k} & =f_{k}+g_{k} u
\end{aligned}
$$

where $x \in \mathbb{R}^{n}$, and $z_{1}, z_{2}, \cdots, z_{k}$ are scalars. Control signal is presented by $u$, and $f_{k}$ and $g_{k}$ are functions $(k=1,2, \cdots, n)$. In each equation state variable $z_{k}$ is fed back as an input to $z_{k-1}$. The objective is to stabilize the system (3.1-3.5) using backstepping control to steer $x$ to its desired value, there $z_{1}$ stabilizes (3.1), $z_{2}$ stabilizes (3.2), $z_{3}$ stabilizes (3.3). $u$ finally stabilizes (3.5) and hence the overall system. For simplicity of explanation and notation, consider a system with $n=2$ :

$$
\begin{aligned}
\dot{x} & =f_{0}+g_{0} z_{1} \\
\dot{z_{1}} & =f_{1}+g_{1} z_{2}
\end{aligned}
$$

The objective is to stabilize $(3.6-3.7)$ with $u$ through backstepping procedure. Start with scalar subsystem (3.6). This equation is stabilized through input $z_{1}$. Stability of this scalar equation is guaranteed with a suitable candidate Lyapunov function. Stabilizing state feedback controller is $z_{1}=\phi_{0}(x)$, which is zero at the origin and the Lyapunov function $V_{0}=\frac{1}{2} x^{2}$ that satisfies stability criteria [60]

$$
\frac{\partial V_{0}}{\partial x}\left[f_{0}(x)+g_{0}(x) \phi_{0}(x)\right] \leq-W(x)
$$

where $W(x)$ is a positive definite function. Applying a transformation on (3.7), using virtual control $u_{1}$ yields

$$
z_{2}=\frac{1}{g_{1}}\left[u_{1}-f_{1}\right]
$$


It is assumed that $g_{1} \neq 0$ over the domain of interest. Transformation (3.9) reduces (3.6-3.7) into (3.6) plus a pure integrator

$$
\begin{aligned}
\dot{x} & =f_{0}+g_{0} z_{1} \\
\dot{z}_{1} & =u_{1}
\end{aligned}
$$

where $u_{1}$ has to stabilize (3.10-3.11). Adding and subtracting $g \phi_{0}(x)$ on the right hand side of (3.10) yields

$$
\dot{x}=\left[f_{0}+g_{0} \phi_{0}(x)\right]+g_{0}\left[z_{1}-\phi_{0}(x)\right]
$$

substituting the new variable

$$
v=z_{1}-\phi_{0}(x)
$$

in (3.12) yields

$$
\dot{x}=\left[f_{0}+g_{0} \phi_{0}(x)\right]+g_{0} v
$$

taking the derivative of (3.13) yields

$$
\dot{v}=u_{1}-\dot{\phi}_{0}(x)
$$

and this can be seen as backstepping $-\phi_{0}(x)$ through the integrator. Defining a new variable $w=\dot{v}$, the system becomes

$$
\begin{aligned}
\dot{x} & =\left[f_{0}+g_{0} \phi_{0}(x)\right]+g_{0} v \\
\dot{v} & =w
\end{aligned}
$$

Equations (3.16-3.17) are similar to the system of equations (3.10-3.11). Schematic block diagram Figure 3.1 shows the backstepping control design. The Figures (3.1) (a) and (d) have similar structures. Except that in (d) the first component has asymptotically stable origin. 
(a)

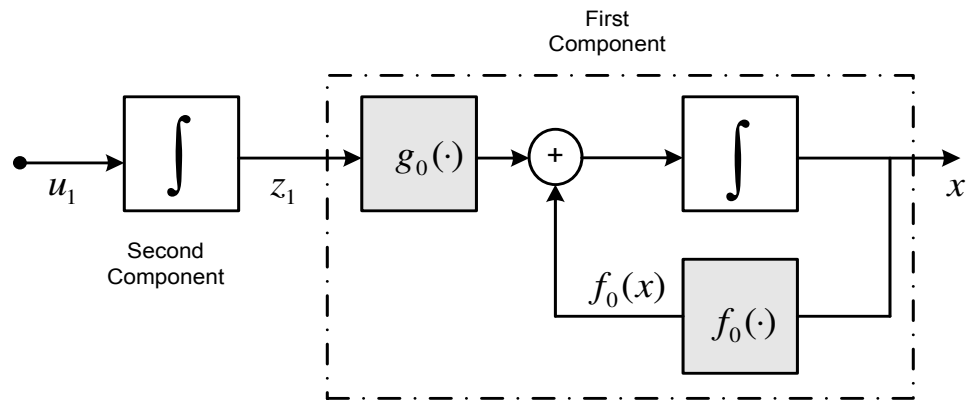

(b)

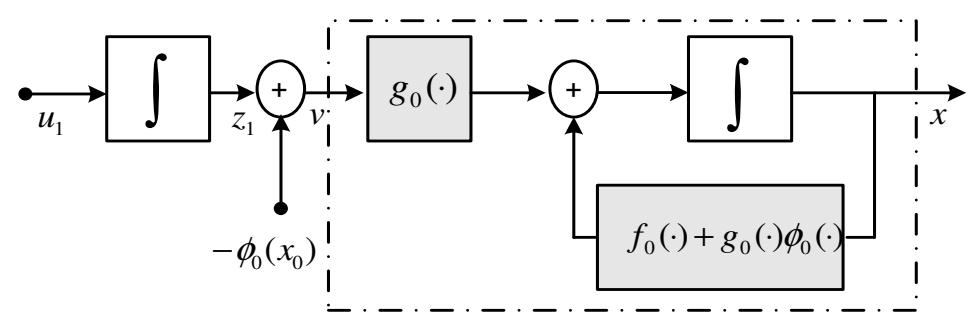

(c)

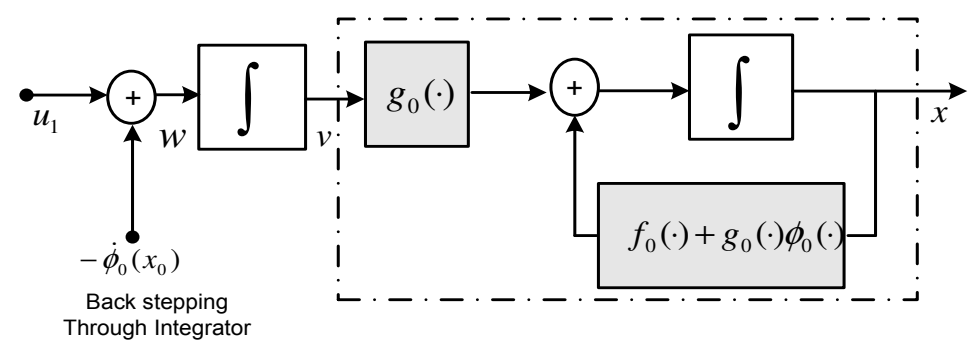

(d)

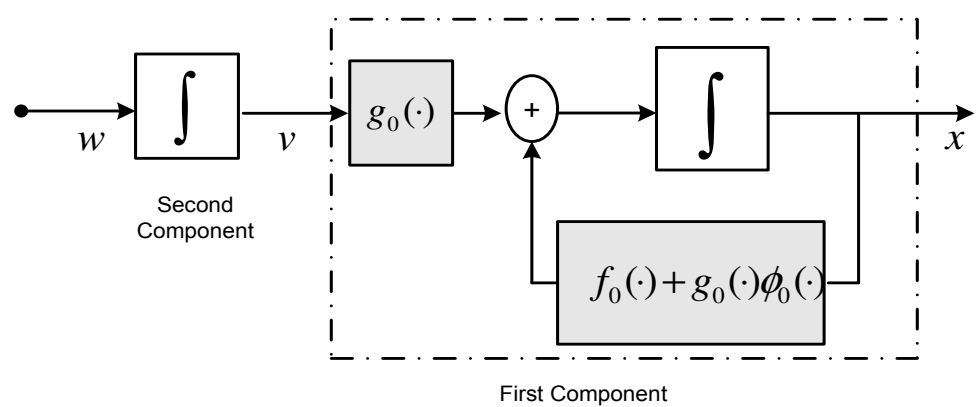

Figure 3.1: Backstepping Design Procedure 
A Lyapunov function candidate for (3.16-3.17) is

$$
V_{1}\left(x, z_{1}\right)=V_{0}(x)+\frac{1}{2} v^{2}
$$

and its derivative (using (3.8))

$$
\begin{array}{r}
\dot{V}_{1}\left(x, z_{1}\right)=\frac{\partial V_{0}}{\partial x}\left[f_{0}+g_{0} \phi_{0}(x)\right]+\frac{\partial V_{0}}{\partial x} g_{0} v+v w \\
\dot{V}_{1}\left(x, z_{1}\right) \leq-W(x)+\frac{\partial V_{0}}{\partial x} g_{0} v+v w
\end{array}
$$

Choosing a stabilizing control law $w$ in a way to achieve negative definiteness of $\dot{V}_{1}\left(x, z_{1}\right)$

$$
w=-\frac{\partial V_{0}}{\partial x} g_{0}-k_{1} v, \quad k_{1}>0
$$

(3.20) becomes

$$
\dot{V}_{1}\left(x, z_{1}\right) \leq-W(x)-k_{1} v^{2}
$$

This choice stabilizes the origin $(x=0, v=0)$ globally and asymptotically. Explicit equation for virtual control is obtained by substituting $w$ in (3.11) and (3.15).

$$
u_{1}=\frac{\partial \phi_{0}}{\partial x}\left[f_{0}+g_{0} z_{1}\right]-\frac{\partial V_{0}}{\partial x} g_{0}-k_{1}\left[z_{1}-\phi_{0}(x)\right]
$$

From (3.9), final control is obtained

$$
z_{2}=\phi_{1}\left(x, z_{1}\right)=\frac{1}{g_{1}}\left[\frac{\partial \phi_{0}}{\partial x}\left[f_{0}+g_{0} z_{1}\right]-\frac{\partial V_{0}}{\partial x} g_{0}-k_{1}\left[z_{1}-\phi_{0}(x)\right]-f_{1}\right], k_{1}>0
$$

Lyapunov function for (3.6)-(3.7)

$$
V_{1}\left(x, z_{1}\right)=V_{0}(x)+\frac{1}{2}\left[z_{1}-\phi_{0}(x)\right]^{2}
$$

With $z_{2}=\phi_{1}\left(x, z_{1}\right)$ determined, it is easy to extend this technique to a larger number of equations (3.1-3.3).

For larger system include (3.3) into (3.1)-(3.2). In compact notation

$$
\begin{aligned}
& \dot{Z}_{1}=F_{1}+G_{1} z_{2} \\
& \dot{z}_{2}=f_{2}+g_{2} z_{3}
\end{aligned}
$$

where $Z_{1}, F_{1}, G_{1}$ are vectors:

$$
Z_{1}=\left[\begin{array}{c}
x \\
z_{1}
\end{array}\right] \quad F_{1}=\left[\begin{array}{c}
f_{0}+g_{0} z_{1} \\
f_{1}
\end{array}\right] \quad G_{1}=\left[\begin{array}{c}
0 \\
g_{1}
\end{array}\right]
$$


The structure of (3.26)-(3.27) is identical to that of (3.6)-(3.7). Therefore, the same steps can be repeated by introducing Lyapunov function

$$
V_{2}\left(x, z_{1}, z_{2}\right)=V_{1}\left(x, z_{1}\right)+\frac{1}{2}\left[z_{2}-\phi_{1}\left(x, z_{1}\right)\right]^{2}
$$

The final control law for the system (3.26-3.27) will become

$$
\begin{gathered}
z_{3}=\phi_{2}\left(x, z_{1}, z_{2}\right)=\frac{1}{g_{2}}\left[\frac{\partial \phi_{1}}{\partial Z_{1}}\left(F_{1}+G_{1} z_{2}\right)-\frac{\partial V_{1}}{\partial Z_{1}} G_{1}-k_{2}\left(z_{2}-\phi_{1}\right)-f_{2}\right] \\
=\frac{1}{g_{2}}\left\{\left[\begin{array}{ll}
\frac{\partial \phi_{1}}{\partial x} & \frac{\partial \phi_{1}}{\partial z_{1}}
\end{array}\right]\left(\left[\begin{array}{c}
f_{0}+g_{0} z_{1} \\
f_{1}
\end{array}\right]+\left[\begin{array}{c}
0 \\
g_{1}
\end{array}\right] z_{2}\right)-\left[\begin{array}{cc}
\frac{\partial V_{1}}{\partial x} & \frac{\partial V_{1}}{\partial z_{1}}
\end{array}\right]\left[\begin{array}{c}
0 \\
g_{1}
\end{array}\right]-k_{2}\left(z_{2}-\phi_{1}\right)-f_{2}\right\}
\end{gathered}
$$

where $k_{2}>0$ and simplified as

$$
\begin{aligned}
z_{3} & =\frac{1}{g_{2}}\left[\frac{\partial \phi_{1}}{\partial x}\left(f_{0}+g_{0} z_{1}\right)+\frac{\partial \phi_{1}}{\partial z_{1}}\left(f_{1}+g_{1} z_{2}\right)-\frac{\partial V_{1}}{\partial z_{1}} g_{1}-k_{2}\left(z_{2}-\phi_{1}\right)-f_{2}\right], k_{2}>0 \\
z_{3} & =\phi_{2}\left(x, z_{1}, z_{2}\right)
\end{aligned}
$$

Clearly the backstepping control technique can be applied to system to an $n^{\text {th }}$ order system, providing the system is in the correct form of strict feedback.

\subsubsection{Power System Problem Formulation}

In general the complete differential-algebraic model of any power system has the following form:

$$
\begin{aligned}
\dot{x} & =f(x, V) \\
Y V & =I(x, V)
\end{aligned}
$$

where the parameters are denoted as:

- $x$ - state vector of power system model

- $V$ - bus voltage vector of the system

- $I$ - current injection vector into the system

- $Y$ - Admittance matrix, including constant impedance loads and the modifications due to the faults 
Initially $\dot{x}=0$ and $Y V_{0}=I\left(x_{0}, V_{0}\right)$. Both functions $f(x, V)$ and $I(x, V)$ are nonlinear functions and can be computed if the operating condition $(x, V)$ is given. $I(x, V)$ is a current injection vector, which includes currents from all dynamic and nonlinear static devices, and $Y$ is the admittance matrix, which includes constant impedance loads. In particular dynamic of synchronous generator is explained in following.

The dynamics of a real electric power system is complex due to large system components, their variety and their interconnection. Mathematical formulation is governed by differential algebraic equations that describes the dynamics of the generators in connection with the grid. Here the main concern is transient stability and fast acting control design. The $i^{\text {th }}$ generator in an n-machine system is modeled by the following set of differential and algebraic equations [81]. Throughout this study, the following two-axis transient synchronous generator model is considered:

a) Differential Equations

$$
\begin{aligned}
\dot{\delta}_{i} & =\omega_{i}-\omega_{0} \\
\dot{\omega}_{i} & =-\frac{D_{i}}{2 H_{i}}\left(\omega_{i}-\omega_{0}\right)+\frac{\omega_{0}}{2 H_{i}}\left(P_{m i}-P_{e i}\right) \\
\dot{E}_{d i}^{\prime} & =\frac{1}{T_{q o i}^{\prime}}\left[-I_{q i}\left(X_{q i}-X_{q i}^{\prime}\right)-E_{d i}^{\prime}\right] \\
\dot{E}_{q i}^{\prime} & =\frac{1}{T_{d o i}^{\prime}}\left[-E_{q i}^{\prime}+I_{d i}\left(X_{d i}-X_{d i}^{\prime}\right)+E_{f l d i}\right]
\end{aligned}
$$

b) Algebraic Equations

$$
\begin{gathered}
P_{e i}=E_{d i}^{\prime} I_{d i}+E_{q i}^{\prime} I_{q i} \\
E_{d i}=E_{d i}^{\prime}+I_{q i} X_{d i}^{\prime} \\
E_{q i}=E_{q i}^{\prime}-I_{d i} X_{d i}^{\prime} \\
I_{q i}=\sum_{j=1}^{n} E_{q j}^{\prime}\left(B_{i j} \sin \left(\delta_{i}-\delta_{j}\right)+G_{i j} \cos \left(\delta_{i}-\delta_{j}\right)\right) \\
I_{d i}=\sum_{j=1}^{n} E_{q j}^{\prime}\left(G_{i j} \sin \left(\delta_{i}-\delta_{j}\right)-B_{i j} \cos \left(\delta_{i}-\delta_{j}\right)\right)
\end{gathered}
$$

The subsystem dynamics for each generator are nonlinear and coupled through nonlinear coupling currents $I_{d i}$ and $I_{q i} . \quad B_{i j}$ and $G_{i j}$ are elements of susceptance and conductance 
Table 3.1: Synchronous generator and transmission line variables

\begin{tabular}{rll}
\hline$\delta_{i}$ & $\ldots$ & Rotor angle in radians \\
$\omega_{i}$ & $\ldots$ & Speed in radians per second \\
$\omega_{0}$ & $\ldots$ & Rated speed in radians per second \\
$E_{f l d}$ & $\ldots$ & Excitation field voltage \\
$P_{e i}$ & $\ldots$ & Active power in per unit \\
$Q_{e i}$ & $\ldots$ & Reactive power in per unit \\
$H_{i}$ & $\ldots$ & Inertia constant in seconds \\
$D_{i}$ & $\ldots$ & Damping constant in per unit \\
$P_{m i}$ & $\ldots$ & Mechanical power \\
$I_{d i}$ & $\ldots$ & Direct axis current \\
$I_{q i}$ & $\ldots$ & Quadrature axis current \\
$E_{q i}^{\prime}$ & $\ldots$ & Transient EMF in quadrature axis \\
$E_{d i}^{\prime}$ & $\ldots$ & Transient EMF in direct axis \\
$E_{q i}$ & $\ldots$ & EMF in quadrature axis \\
$E_{d i}$ & $\ldots$ & EMF in direct axis \\
$T_{d o}^{\prime}$ & $\ldots$ & Direct open circuit time constant \\
$T_{q o}^{\prime}$ & $\ldots$ & Quadrature open circuit time constant \\
$X_{q}$ & $\ldots$ & Quadrature axis reactance \\
$X_{d}$ & $\ldots$ & Direct axis reactance \\
$X_{d}^{\prime}$ & $\ldots$ & Direct axis transient reactance \\
$X_{q}^{\prime}$ & $\ldots$ & Quadrature axis transient reactance \\
$B_{i j}$ & $\ldots$ & Elements of susceptance matrix \\
$G_{i j}$ & $\ldots$ & Elements of conductance matrix \\
\hline & &
\end{tabular}

matrix respectively. Synchronous generator and transmission line variable are given in Table 3.1. Since the dynamic of generator is not in strict feedback form, the alternative state as active power is considered. In following the simplification of generator model into strict feedback form is presented. An alternative state, active power, is used instead of $E_{d i}^{\prime}$ and $E_{q i}^{\prime}$ which are internal generator voltages from their relation in algebraic form. Dynamic equation for active 
power is obtained by taking derivative of (3.35) and using (3.33) and (3.34).

$$
\begin{aligned}
& \dot{P}_{e i}=\frac{1}{T_{q o i}^{\prime}}\left[-I_{q i}\left(X_{q i}-X_{q i}^{\prime}\right)-E_{d i}^{\prime}\right] I_{d i}+E_{d i}^{\prime} \dot{I}_{d i}+ \\
& +\frac{1}{T_{d o i}^{\prime}}\left[-E_{q i}^{\prime}+I_{d i}\left(X_{d i}-X_{d i}^{\prime}\right)+E_{f l d i}\right] I_{q i}+E_{q i}^{\prime} \dot{I}_{q i}
\end{aligned}
$$

Equation (3.31), (3.32) and (3.44) are dynamic equations for generator. The following state variables are introduced by shifting the equilibrium point to the origin,

$$
\begin{aligned}
\Delta \delta_{i} & =\delta_{i}-\delta_{i 0} \\
\Delta \omega_{i} & =\omega_{i}-\omega_{0} \\
\Delta P_{e i} & =P_{e i}-P_{m i}^{\circ}
\end{aligned}
$$

since there is no turbine-governor dynamics then $P_{m i}^{\circ}=P_{e i}^{\circ}$ and $\Delta \dot{P}_{e i}=\dot{P}_{e i}$. Equation (3.44) is written as

$$
\begin{gathered}
\Delta \dot{P}_{e i}=\frac{1}{T_{q o i}^{\prime}}\left[-I_{q i}\left(X_{q i}-X_{q i}^{\prime}\right)-E_{d i}^{\prime}\right] I_{d i}+E_{d i}^{\prime} \dot{I}_{d i}+ \\
+\frac{1}{T_{d o i}^{\prime}}\left[-E_{q i}^{\prime}+I_{d i}\left(X_{d i}-X_{d i}^{\prime}\right)+E_{f l d i}\right] I_{q i}+E_{q i}^{\prime} \dot{I}_{q i}
\end{gathered}
$$

substitute $-E_{d i}^{\prime} I_{d i}=E_{q i}^{\prime} I_{q i}-P_{e i}$

$$
\begin{array}{r}
\Delta \dot{P}_{e i}=-\frac{P_{e i}}{T_{q o i}^{\prime}}-\frac{I_{q i} I_{d i}\left(X_{q i}-X_{q i}^{\prime}\right)}{T_{q o i}^{\prime}}+E_{q i}^{\prime} I_{q i}\left(\frac{1}{T_{q o i}^{\prime}}-\frac{1}{T_{d o i}^{\prime}}\right)+ \\
+E_{d i}^{\prime} \dot{I}_{d i}+E_{q i}^{\prime} \dot{I}_{q i}+\frac{E_{f l d_{i}} I_{q i}}{T_{d o i}^{\prime}}+\frac{I_{q i} I_{d i}\left(X_{d i}-X_{d i}^{\prime}\right)}{T_{d o i}^{\prime}}
\end{array}
$$

replace $P_{e i}=\Delta P_{e i}+P_{e i}^{\circ}$

$$
\begin{array}{r}
\Delta \dot{P_{e i}}=-\frac{\Delta P_{e i}}{T_{q o i}^{\prime}}-\frac{I_{q i} I_{d i}\left(X_{q i}-X_{q i}^{\prime}\right)}{T_{q o i}^{\prime}}+E_{q i}^{\prime} I_{q i}\left(\frac{1}{T_{q o i}^{\prime}}-\frac{1}{T_{d o i}^{\prime}}\right)-\frac{P_{e i}^{\circ}}{T_{q o i}^{\prime}}+ \\
+E_{d i}^{\prime} \dot{I}_{d i}+E_{q i}^{\prime} \dot{I}_{q i}+\frac{E_{f l d_{i}} I_{q i}}{T_{d o i}^{\prime}}+\frac{I_{q i} I_{d i}\left(X_{d i}-X_{d i}^{\prime}\right)}{T_{d o i}^{\prime}}
\end{array}
$$

adding and subtracting a term $\frac{E_{f l d_{i}}^{\circ} I_{q i}}{T_{d o i}^{\prime}}$

$$
\begin{aligned}
\Delta \dot{P}_{e i}= & -\frac{\Delta P_{e i}}{T_{q o i}^{\prime}}-\frac{I_{q i} I_{d i}\left(X_{q i}-X_{q i}^{\prime}\right)}{T_{q o i}^{\prime}}+E_{q i}^{\prime} I_{q i}\left(\frac{1}{T_{q o i}^{\prime}}-\frac{1}{T_{d o i}^{\prime}}\right)-\frac{P_{e i}^{\circ}}{T_{q o i}^{\prime}}+ \\
& +E_{d i}^{\prime} \dot{I}_{d i}+E_{q i}^{\prime} \dot{I}_{q i}+\frac{\Delta E_{f l d_{i}} I_{q i}}{T_{d o i}^{\prime}}+\frac{I_{q i} I_{d i}\left(X_{d i}-X_{d i}^{\prime}\right)}{T_{d o i}^{\prime}}+\frac{E_{f l d_{i}}^{\circ} I_{q i}}{T_{d o i}^{\prime}}
\end{aligned}
$$


Dynamic equations will become

$$
\begin{aligned}
& \Delta \dot{\delta}_{i}=\Delta \omega_{i} \\
& \Delta \dot{\omega}_{i}=-\frac{D_{i}}{2 H_{i}} \Delta \omega_{i}-\frac{\omega_{o}}{2 H_{i}} \Delta P_{e i} \\
& \Delta \dot{P}_{e i}=-\frac{\Delta P_{e i}}{T_{q o i}^{\prime}}-\frac{I_{q i} I_{d i}\left(X_{q i}-X_{q i}^{\prime}\right)}{T_{q o i}^{\prime}}+E_{q i}^{\prime} I_{q i}\left(\frac{1}{T_{q o i}^{\prime}}-\frac{1}{T_{d o i}^{\prime}}\right)-\frac{P_{e i}^{\circ}}{T_{q o i}^{\prime}}+ \\
& +E_{d i}^{\prime} \dot{I}_{d i}+E_{q i}^{\prime} \dot{I}_{q i}+\frac{I_{q i} I_{d i}\left(X_{d i}-X_{d i}^{\prime}\right)}{T_{d o i}^{\prime}}+\frac{E_{f l d_{i}}^{\circ} I_{q i}}{T_{d o i}^{\prime}}+\frac{\Delta E_{f l d_{i}} I_{q i}}{T_{d o i}^{\prime}}
\end{aligned}
$$

The controller will be designed based on (3.46), (3.47), and (3.48). Electromagnetic equation (3.48) is coupled with mechanical equations (3.46), (3.47). In contrast to the mechanical equations, there are different ways to model electromagnetic models, depending primarily upon the time scale at which the phenomenon of interest occurs. Former formulation provides good agreement for the behavior of the controller for synchronous generator over a time scale of transient stability. Providing states and control input:

$$
x=\Delta \delta \quad z_{1}=\Delta \omega \quad z_{2}=\Delta P_{e} \quad u=\Delta E_{f l d}
$$

and functions correspond to

$$
\begin{array}{r}
g_{0}=1 \quad g_{1}=-\frac{\omega_{0}}{2 H} \quad g_{2}=\frac{I_{q}}{T_{d o}^{\prime}} \quad f_{0}=0 \quad f_{1}=-\frac{D}{2 H} \Delta \omega \\
f_{2}=-\frac{\Delta P_{e}}{T_{q o}^{\prime}}-\frac{I_{q} I_{d}\left(X_{q}-X_{q}^{\prime}\right)}{T_{q o}^{\prime}}+E_{q}^{\prime} I_{q}\left(\frac{1}{T_{q o}^{\prime}}-\frac{1}{T_{d o}^{\prime}}\right)-\frac{P_{e i}^{\circ}}{T_{q o}^{\prime}}+ \\
+E_{d}^{\prime} \dot{I}_{d}+E_{q}^{\prime} \dot{I}_{q}+\frac{I_{q} I_{d}\left(X_{d}-X_{d}^{\prime}\right)}{T_{d o}^{\prime}}+\frac{E_{f l d_{i}}^{\circ} I_{q i}}{T_{d o i}^{\prime}}
\end{array}
$$

Equations are in strict feedback form and suitable for backstepping controller design (3.30). The final control signal is obtained as

$$
\begin{aligned}
u=\frac{T_{d o}^{\prime}}{I_{q}} & {\left[\left(1+k_{1}\right) \frac{2 H}{\omega_{0}}\left(f_{0}+g_{0} \Delta \omega\right)+\left(1+k_{1}-\frac{D}{2 H}\right) \frac{2 H}{\omega_{0}}\left(f_{1}+g_{1} \Delta P_{e}\right)-(\Delta \delta+\Delta \omega)-\right.} \\
& \left.-k_{2} \Delta P_{e}-k_{2} \frac{2 H}{\omega_{0}}\left[\Delta \omega\left(-1-k_{1}+\frac{D}{2 H}\right)-\Delta \delta\left(-1-k_{1}\right)\right]-f_{2}\right] \quad k_{1}>0, k_{2}>0
\end{aligned}
$$

Controller gains are $k_{1}$ and $k_{2}$. Note that: 
- The nonlinear control law (3.51) is not feasible if $I_{q}=0$. This condition occurs when rotor angle is $\delta=n \times 180^{\circ}$ with $n$ presents an integer number. Normally, when rotor angle reaches $180^{\circ}$, it is almost impossible to maintain stability by using incorporating excitation control [9]. A reasonable system working region for rotor angle is $0^{\circ}<\delta<$ $180^{\circ}$.

- Equations in strict feedback form has an advantage of formulation where interconnection term appears only on last equation (3.48). Note that interface term is

$$
\begin{aligned}
d_{i}=- & \frac{I_{q} I_{d}\left(X_{q}-X_{q}^{\prime}\right)}{T_{q o}^{\prime}}+E_{q}^{\prime} I_{q}\left(\frac{1}{T_{q o}^{\prime}}-\frac{1}{T_{d o}^{\prime}}\right)-\frac{P_{e i}^{\circ}}{T_{q o}^{\prime}}+ \\
& +E_{d}^{\prime} \dot{I}_{d}+E_{q}^{\prime} \dot{I}_{q}+\frac{I_{q} I_{d}\left(X_{d}-X_{d}^{\prime}\right)}{T_{d o}^{\prime}}+\frac{E_{f l d_{i}}^{\circ} I_{q i}}{T_{d o i}^{\prime}}
\end{aligned}
$$

- Various factors affect the transient stability of a system, such as strength of transmission system and connection with the grid, characteristics of generators and their controllers. Another important factor is the fault duration and the time span in which the faulted line and equipment can be disconnected and restored back.

\subsubsection{Case Studies}

\section{Single Machine Infinite Bus}

A certain portion of the system including generator, actuators, and transmission lines is the focus of the case study. The remainder of the system is represented by a simplified model called dynamic equivalent. It is assumed that the remainder of the system is very large in comparison with the concerned generator and adequate to represented by infinite bus. The model consists of single generator connected through two parallel transmission lines to infinite bus [65]. Single line diagram is shown in Figure 3.2.

A case study is presented to illustrate the effectiveness of the proposed controller during and after contingency in the system. The results are compared with conventional Power System Stabilizer. Figure 3.2 shows schematic diagram of single machine infinite bus. Complete data related to this system is given in Appendix A. The objective is to improve transient stability by implementing excitation controller. 


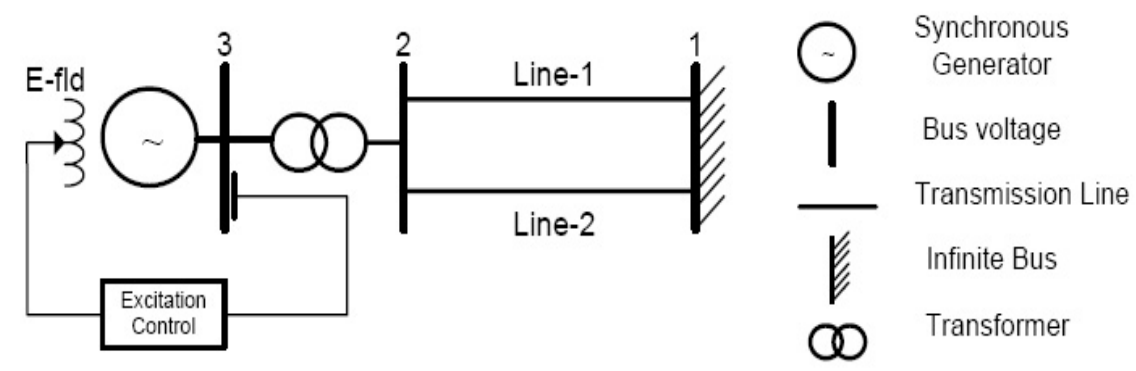

Figure 3.2: Single Machine Infinite Bus System

\section{Excitation System Control Design}

Supplementary control signal is used to add damping to system oscillations. This signal is applied through excitation type- $A C 4 A[42]$. Following control strategies are analyzed:

- Backstepping controller is implemented on generator. Control gains are obtained by trial and error $k_{1}=1$ and $k_{2}=4.6$.

- PSS with excitation type- $A C 4 A$, whose transfer functions are given below, is implemented on generator. PSS has two stage lead-lag with a wash-out stage [9].

$$
K_{w} \frac{s T_{w}}{1+s T_{w}} \frac{\left(1+s T_{1}\right)^{2}}{\left(1+s T_{2}\right)^{2}}=13.7750 \frac{s 1.410}{1+s 1.410} \frac{(1+s 0.154)^{2}}{(1+s 0.033)^{2}}
$$

- Excitation type- $A C 4 A$ with no supplementary signal

\section{Scenario}

Three phase fault happens on first transmission line at 0.5 second, the fault is removed at $0.57 \mathrm{sec}$. The fault is cleared and transmission line is reconnected at 0.65 seconds. Results are shown in Figures 3.3 and 3.4. Proposed controller effectively damps the oscillation and modulates the voltage. Better performance is achieved in comparison with PSS. For such small system the trial and error parameter search may be possible but for large system with more control gains, trial and error will not be practical. Therefore, a systematic way should presented to obtain control gains and satisfy objective function of the system.

In the following, the effect of $k_{1}$ and $k_{2}$ on suppressing oscillation are investigated. Even for this small system, the effect of control gains on damping oscillation is significant. Figure 3.5 shows the relative rotor angle and speed deviation of generator for several $k_{1}, k_{2}$ pairs (Table 3.2). Unstable scenario (dot-line) happens when backstepping parameters become negative 

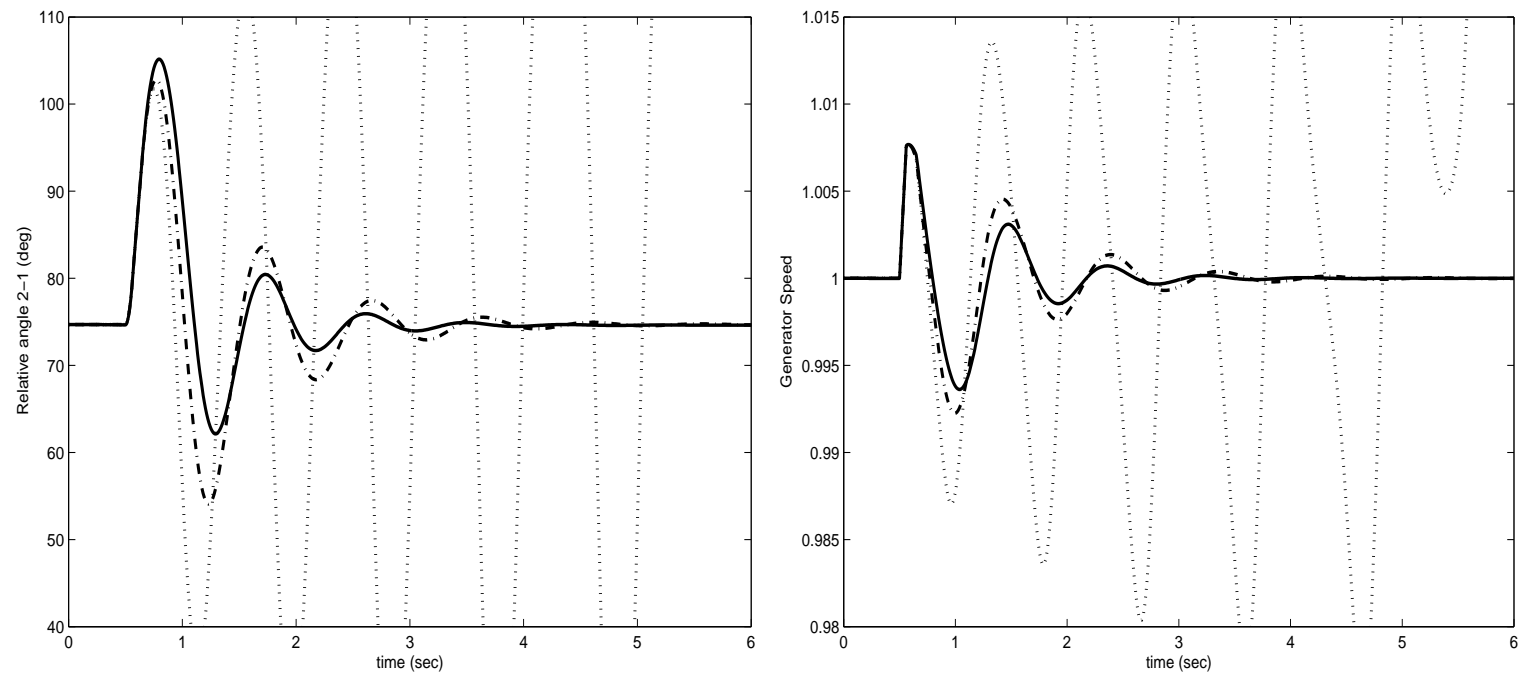

Figure 3.3: Relative rotor angle (deg), Generator's shaft speed (p.u.). Backstepping (solid line -), PSS (dash-dot line -.) and uncontrolled system (dotted line :)
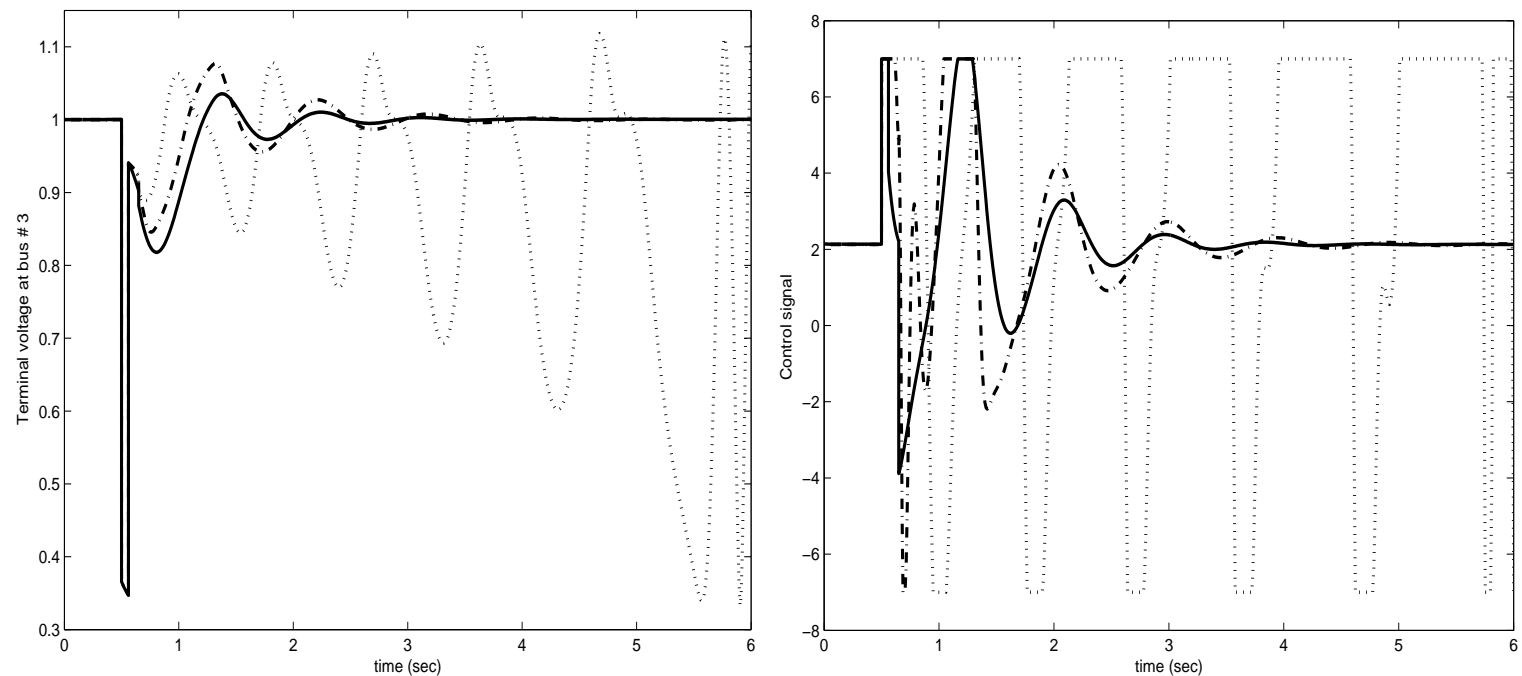

Figure 3.4: Terminal voltage at bus 3 (p.u.), and Control signal (p.u.). Backstepping (solid line -), PSS (dash-dot line -.) and uncontrolled system (dotted line :)

and Lyapunov stability criteria are not satisfied. From the Figures 3.5 and 3.6, unstability (dotted line) is in from of aperiodic drift due to non-tuned controller gains which results in insufficient synchronizing torque. As a result a large excursion of rotor angle beyond the first swing occurs and generator becomes unstable. 

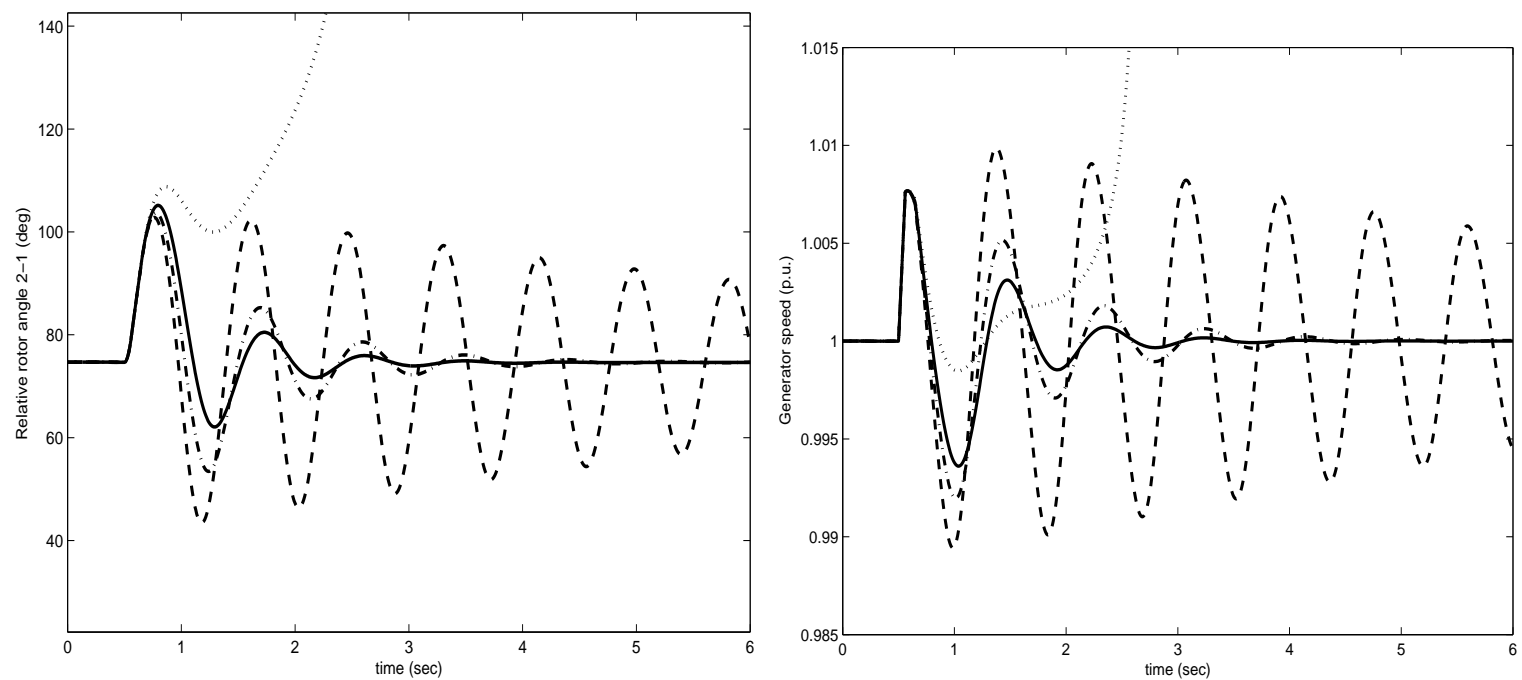

Figure 3.5: Relative rotor angle (deg) and Generator's shaft speed (p.u.)
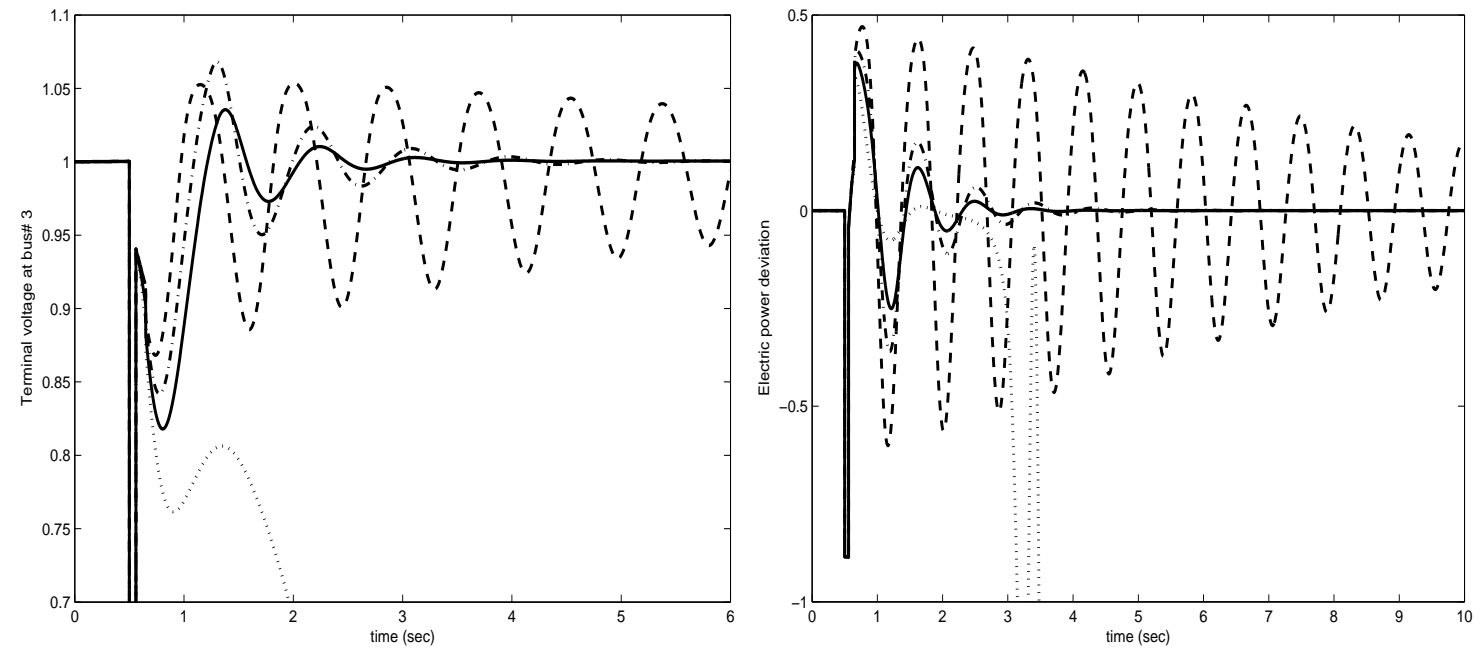

Figure 3.6: Terminal voltage at bus 3 (p.u.) and electric power deviation of generator (p.u.)

Table 3.2: Backstepping Control Gains $k_{1}, k_{2}$

\begin{tabular}{c|c|c|c|c}
\hline Control Parameters & solid line - & dotted line . & dash dot line.- & dash line -- \\
\hline$k_{1}$ & 1 & -2 & 3 & 6 \\
$k_{2}$ & 4.6 & 5 & 5.7 & 3 \\
\hline
\end{tabular}




\subsection{Function Optimization}

\subsubsection{Introduction}

Organization of this section is as follows: Subsection 3.2.2 gives an overview of function optimization problem in general. An evolutionary search technique known as Particle Swarm Optimization (PSO), is described in Subsection 6.4.

\subsubsection{Optimization Problem}

Optimization is defined as finding the best possible solution to a problem given a set of constraints. Problem with two or more objective functions is called multi objective optimization problem. Most practical problems require simultaneous optimization of multiple objectives. Usually, these objectives have adverse effect on each other that can not be optimized one at a time. Simultaneous function optimization with evolutionary search algorithms can find solutions to all non-continuous and non-linear problems. This approach has become common in solving these types of problems. Numerous applications of evolutionary algorithms can be found especially in electric power systems [7]. The flexibility of evolutionary algorithms to address optimization problem using any reasonable representation and objective functions gives these techniques an advantage over classical optimization procedures. Given a control structure with a number of adjustable gains, mathematical model of the system, and objective functions, while the aim is to obtain the best values of controllers' gains that optimize objective function subject to system constraints.

Optimization problem is formulated as follow:

$$
\min _{\text {s.t. } g_{i}(\kappa) \leq 0} f(\kappa)
$$

where $\kappa \in R^{m}$ is the optimization parameter, $f(\kappa) \in R^{n}$ is objective function vector, $f_{i}(\kappa)$ for $i=1,2, \cdots, n$ are the objective functions, and $g_{i}(\kappa) \leq 0$ the system constraints. Computational intelligence-based can provide techniques that are feasible and almost independent of the size of problem.

Evolutionary algorithm can be applied to any problem that can be formulated as function optimization (3.54). It requires data structure to represents solutions, performance index to evaluate the solutions, and variations operator to provide new solutions from the old ones. 
Advantage of the evolutionary algorithm comes from the ability in automating and problem solving routines. Genetic algorithm (GA) and PSO are both considered as evolutionary algorithm. Genetic algorithm is a search technique to find approximate solution to optimization technique [59]. The algorithm uses techniques inspired by evolutionary biology such as mutation, cross over, natural selection. Despite obtaining good solutions in hard search spaces, still have some disadvantages such as tendency to converge toward local optima rather than global optimum of the problem, and hard to implement on dynamic data sets. PSO is another evolutionary technique that is not largely affected by nonlinearity and the size of the problem. The technique can easily converge to optimal solution that can be executed in search in solution space for solving multi-objective optimization problems as formulated in (3.54).

Large number of evolutionary algorithms applications, especially for parameter estimation and tuning of control gains can be found in electric power system literature [1], [2]. Among all these techniques PSO has gained increased attention. Some advantages of PSO over other optimization techniques are [19]:

- It has the ability to escape local minima

- It has less parameters to adjust, unlike many others

- It is easy for computer implementation and coding

- It is easy to implement and program with mathematical and logic operations

- It does not require a good initial solution to start the iteration

- It can be used with almost any realistic objective functions i.e. continuous or noncontinuous, convex or non-convex

- It has more effective memory capability (local and neighboring best)

A detailed survey on PSO applications to large scale power systems is covered by Alrashidi and El-Hawary [6]. Recently a comprehensive overview of PSO techniques and different applications in electric power systems are covered by del Valle et. al [24].

\subsubsection{Particle Swarm Optimization Algorithm}

Particle Swarm Optimization is an evolutionary algorithm developed by James Kennedy and Russell Eberhart [59]. The original objective of their research was to mathematically simulate behavior of bird flocks. The search algorithm is based on cooperation and competition among the population members. The objective is to find optimal regions of a complex search space 
through interaction of individuals in a population of particles. Each individual of the population has an adaptable velocity (position change), according to which it moves in the search space. Moreover, each individual has a memory remembering the best position of the search space it has ever visited. Its movement is an aggregated acceleration toward its best previously visited position. Another best value that is tracked by PSO is the best value obtained so far by any particle in the neighborhood of the particle. The main idea is to change the position and velocity of each particle toward global best location at each time step. As a result, after number of iterations the particles among populations are found to have accumulated around one or more of the optima and tends to find the global optima among all.

Given the size of the problem and the system complexities, the solution is assumed to lie in the range of an $\mathrm{N}$-dimensional space, where each potential solution is called a particle. Particle has a position and a velocity and moves in the search space toward an optimal solution. Through the course of this study, PSO is applied to electric power system for tuning nonlinear backstepping controller gains. The particles represent the controllers with this respective gains that are sought [3], [4].

Some definitions are given below:

Particle $K_{i}(t)$ : A candidate solution (controller gains) for $i^{\text {th }}$ controller at iteration $t$.

Population: A set of $n$ particles $\left\{K_{1}(t), K_{2}(t) \cdots K_{n}(t)\right\}$, where $n$ is the number of candidate solutions.

Swarm: Disorganized population of moving particles that tend to gather (with each other), while each particle seems to be moving in a random direction.

Individual best $K_{i}^{*}(t)$ : This is the best value of the objective function $J$ that this particle has ever achieved up to $t^{\text {th }}$ iteration.

$$
\begin{aligned}
K_{i}^{*}(t) & =\left\{K_{i}(t): J_{i}\left(K_{i}^{*}(t)\right) \leq J_{i}\left(K_{i}(\tau)\right), \quad \tau \leq t\right\} \\
J_{i}^{*}(t) & =J_{i}\left(K_{i}^{*}(t)\right)
\end{aligned}
$$

Global best $K^{* *}(t)$ : Among all individual best positions achieved so far, the best position for all particles is called global best.

$$
K_{i}^{* *}(t)=\left\{K_{i}^{*}(t): J\left(K^{* *}(t)\right) \leq J_{i}\left(K_{i}^{*}(t)\right), i=1, \cdots n\right\}
$$

The steps of the PSO algorithm are:

\section{- Step 1: Initialization}


(a) Given a number of controllers $n$, population size $(m)$, maximum number of iterations $t_{\max }$, maximum number of performance evaluation counter $c_{\max }$, admissible range for gains $k_{i, j}^{\min }, k_{i, j}^{\max }$

$$
k_{i, j}^{\min } \leq k_{i, j} \leq k_{i, j}^{\max } i=1,2, \cdots, n, j=1,2, \cdots, m
$$

and number of intervals in $j^{\text {th }}$ dimension $(\mu)$. Set speed range

$$
-\nu_{i, j}^{\max } \leq \nu_{i, j} \leq \nu_{i, j}^{\max }
$$

where

$$
\nu_{i, j}^{\max }=\frac{k_{i, j}^{\max }-k_{i, j}^{\min }}{\mu}
$$

(b) Initialize position and speed using uniformly distributed random numbers, and evaluate the objective function $J_{i}(t)$ of each particle.

(c) Let $J_{i}^{*}(t)=J_{i}(t), K_{i}^{*}(t)=K_{i}(t)$ and

$J^{* *}(t, c)=\min \left[J_{1}^{*}(t) \cdots J_{n}^{*}(t)\right]$

(d) Set $t=t+1, c=c+1$

\section{- Step 2: Velocity Update with constriction factor approach}

Velocity is updated by the following equation $[59]^{2}$ :

$$
\begin{gathered}
\nu_{i, j}(t)=\Psi\left(\nu_{i, j}(t-1)+c_{1} r_{1}\left(k_{i, j}^{*}(t-1)-k_{i, j}(t-1)\right)+c_{2} r_{2}\left(k_{i, j}^{* *}(t-1)-k_{i, j}(t-1)\right)\right) \\
\Psi=\frac{2}{\left|2-\varphi-\sqrt{\varphi^{2}-4 \varphi}\right|} \text { where } \varphi=c_{1}+c_{2}, \varphi>4
\end{gathered}
$$

where positive constants $c_{1}, c_{2}$ are weighting factors. As $\phi>4$ increases above 4.0 , the $\Psi$ gets smaller and the damping effect is more pronounced. Hence the amplitude of the individual

$$
\begin{gathered}
{ }^{2} \underbrace{\text { Particle }}_{\text {new velocity }}=\Psi[\underbrace{\text { Particle }}_{\text {velocity }}+\underbrace{c_{1}}_{\text {weights }} \text { rand } \\
\underbrace{\text { Particle }}_{\text {new position }}=\underbrace{\text { pbest }}_{\text {current position }}-\underbrace{\text { Particle velocity }}_{\text {local best }}+\underbrace{\text { Particle }}_{\text {position }})+\underbrace{c_{2}}_{\text {weights }} \text { Rand }(\underbrace{\text { gbest }}_{\text {global best }}-\underbrace{\text { Particle }}_{\text {position }})]
\end{gathered}
$$


particle's oscillations decrease as it focuses on a previous best point. This formulation for velocity results in particle convergence over time, meaning that the amplitude of individual's particles oscillations are decreased as it focuses over the previous point. $r_{1}, r_{2}$ are uniformly distributed random number between 0 and 1 . In case the velocity violates its range, it will be set to its limit (3.58).

\section{- Step 3: Position Update}

For each particle, update each gain using the velocity equation (3.61)

$$
k_{i, j}(t)=\nu_{i, j}(t)+k_{i, j}(t-1)
$$

Update the position $K_{i}(t)=\left[k_{i 1}, \cdots, k_{i m}\right]$ for $i=1, \cdots n$

\section{- Step 4: Performance Evaluation}

Using the updated position, evaluate the objective function $\left[J_{1}(t) \cdots J_{n}(t)\right]=\left[J_{1}\left(K_{1}(t)\right) \cdots J_{n}\left(K_{n}(t)\right)\right]$.

\section{- Step 5: Individual Best Update}

Find individual best using (3.55), i.e. ,find $J_{i}^{*}(t)$ and the associated $K_{i}^{*}(t)$ for each $i$.

\section{- Step 6: Global Best Update}

Find global best using (3.56), i.e. find $J^{* *}(t, c)=J\left(K^{* *}(t, c)\right)$ and $K^{* *}(t, c)$. If

$$
J^{* *}(t, c) \leq J^{* *}(t-1, c-1)
$$

then the objective function has improved, and the gains are updated, set $c=c+1$ and go to next step. Otherwise, update $J^{* *}(t, c), K^{* *}(t)$ and set $c=0$. Then go to next step.

\section{- Step 7: Stopping criteria}

(a) If the best global solution $J^{* *}(t, c)$ can no longer be improved and the counter has reached its maximum number, $c=c_{\max }$, the optimal solution is then the current $K^{* *}(t)$. Exit. 


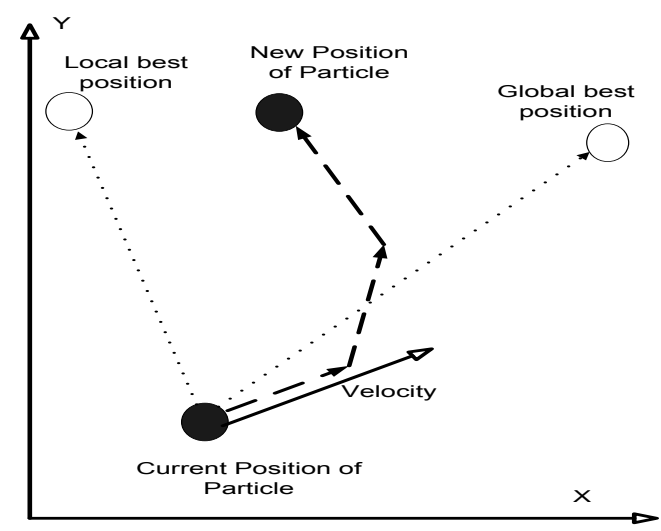

Figure 3.7: Particle movement toward new position

(b) If $t$ reaches $\left(t_{\max }\right)$, the maximum number of allowed iterations has been reached and no feasible solution was found, stop. Otherwise: $t=t+1$ and go to Step 2 .

Schematic view of particle movement is shown in Figure 3.7. Matlab code for PSO algorithm is given in appendix E. Steps for PSO algorithm is given in following:

1. Initialize each particle with random solution in problem domain (initialization)

2. For each particle evaluate the objective function

3. For each particle, calculate the objective function and compare it with its pbest. If the current value of the objective function is better than the pbest then set the value as the pbest and the current position of the particle.

4. Among all pbest, identify the particle that has the best objective function value. The value of the objective function is assigned as gbest with its new position.

5. For each particle update the velocity vector and then the position vector.

6. Repeat steps $2-5$ until stopping criteria are met. These criteria are maximum iteration and minimum error criteria

Parameter Selections: One important issue by implementing the algorithm is how to initialize the population. The positions and velocities of the particles are usually initialized 
randomly [59]. For instance positions are distributed over the dynamic range of each dimension and initial velocities are distributed randomly over the range of minimum and maximum. Where most of the time the maximum value for velocity is specified according to the characteristics of the problem. Note that the velocity of the particle is a stochastic variable and may subject to create an uncontrolled trajectory for the particle. Upper and lower limits are defined for the velocity, in order to damp these oscillations.

$$
\begin{aligned}
\text { if } \nu_{i, j}>\nu_{i, j}^{\max } \quad \text { then } & \nu_{i, j} & =\nu_{i, j}^{\max } \\
\text { if } \quad \nu_{i, j}<-\nu_{i, j}^{\max } & \text { then } & \nu_{i, j}=-\nu_{i, j}^{\max }
\end{aligned}
$$

Based on the characteristic of the problem the value for maximum velocity can be chosen. One common technique to obtain the maximum velocity is formulated in (3.59).

Weighting factors $c_{1}, c_{2}$ control the movement of each particle toward its individual and global best position. Small weights will limit the movement of particle and large weights may cause the particles to diverge. The proper inequality condition is given in (3.62). Note that by considering uneven values for these weights, an uneven cycling for the trajectory of the particle is obtained in searching around an optimum value.

Constriction factor as formulated in (3.62) improves the convergence of the particle over time by damping the oscillations once the particle is focused on the best point in optimal region. And finally a number of particles that is usually around $10-50$. 
Chapter

4

Transient Stability Enhancement Using Excitation Control

\subsection{Power System Stability Enhancement using Backstepping Controller Tuned by Particle Swarm Optimization Tech- nique}

\subsubsection{Introduction}

This section describes an algorithm for function optimization in order to obtain optimum set of gains for backstepping controller. Problem formulation for generator is given in subsection 3.1.3. Controller design, obtained in 3.1.2, is used to stabilize multi-machine power system. The organization of this section is as follows. Subsection 4.1.2 presents the procedure to obtain optimal settings for backstepping controller gains. Three machine nine bus test system is presented in Subsection 4.1.3 for implementing the proposed technique.

\subsubsection{Optimal Settings for Controller Gains}

This subsection describes the procedure for controller tuning. An important aspect of any optimization process is the criterion used to define the goal in system performance, which is 
known as optimization criterion. It is through the selection of this optimization criterion that one can specify the manner in which the system to perform. In following a quadratic performance measure seems appropriate for stability analysis due to contingencies in the system. Optimization problem for selecting the gains for backstepping controller to enhance optimal performance is solving via a Particle Swarm Optimization (PSO). The algorithm has been described in Subsection 6.4.

\section{Objective Function}

Here the goal of PSO procedure is to explore the search space and obtain optimum gains that minimize the defined objective function. Once the optimum controller gains are obtained, controller signal is implemented. In this setting, each backstepping controller has two gains. In multi-machine system gains for the controller are presented as $k_{i, j}$ is used, where $i$ stands for number of controllers and $j$ refers to the sequence number of gains for each controller. The problem is formulated in form of constrained optimization by minimizing objective functions (4.1-4.3). Three different functions are considered for optimizations which are listed in the following. $\Delta \omega_{i}$ is speed deviation, $\Delta \delta_{i j}$ relative rotor angle deviation, and $\Delta V_{\text {gen }_{i}}$ generator terminal voltage deviation. $\alpha, \beta, \gamma$ are weighting factors in optimization problem.

- Objective function I

$$
\min _{k_{i, j}} \sum J_{i}=\sum \int_{0}^{t} \alpha\left(\Delta \omega_{i}\right)^{2} d t
$$

- Objective function II

$$
\min _{k_{i, j}} \sum J_{i}=\sum \int_{0}^{t}\left[\alpha\left(\Delta \omega_{i}\right)^{2}+\gamma\left(\Delta V_{\text {gen }_{i}}\right)^{2}\right] d t
$$

- Objective function III

$$
\min _{k_{i, j}} \sum J_{i}=\sum \int_{0}^{t}\left[\alpha\left(\Delta \omega_{i}\right)^{2}+\beta\left(\sum \Delta \delta_{i j}\right)^{2}+\gamma\left(\Delta V_{g_{e n}}\right)^{2}\right] d t
$$

with same constraints as subject to

$$
\begin{array}{r}
k_{i, j_{\min }}<k_{i, j}<k_{i, j_{\max }} \\
\Delta \omega_{i_{\min }}<\Delta \omega_{i}<\Delta \omega_{i_{\max }} \\
\Delta \delta_{i j_{\min }}<\Delta \delta_{i j}<\Delta \delta_{i j_{\max }}
\end{array}
$$




$$
\Delta V_{\text {gen }_{i_{\text {min }}}}<\Delta V_{\text {gen }_{i}}<\Delta V_{\text {gen }_{i_{\max }}}
$$

Equations (4.1-4.3) present a PSO objective functions subject to set of constraints to obtain optimum gains for each generator as controlled by (3.51). More weight $(\alpha)$ is assigned to $\Delta \omega$ in comparison with $\beta, \gamma, \kappa$ which has ratio of $\frac{\alpha}{\beta}=\frac{\alpha}{\gamma}=100$.

\section{Assumptions}

Objective function (4.3) requires remote signals $\left(\sum \Delta \delta_{i j}\right)$ and can not be computed locally unlike (4.1-4.2). Performance of proposed controller with different objective functions is compared. Design of objective function is not unique and depends on designer choice. Three different objective functions are considered in Sections 4.2 and 4.3. Here, in selecting the controller gains, the main objective is to minimize speed deviation, angle deviation, and terminal voltage deviations from their nominal.

\subsubsection{Case Study}

\section{Three Machine Nine Bus Test System}

Three machine nine bus test system is considered in this case study. Detailed of the data are provided in [9] and also presented in Appendix B. Single line of the system is shown in Figure 4.1.

\section{Excitation System Control Design}

Generators' excitation systems, type- $A C 4 A$, based on fast acting power electronic exciters are considered [42] (Figure 4.2). A voltage regulator with gain $K_{R}=1$ and time constant $T_{R}=0.01$ is added. A lead-lag stage is used to model equivalent time constant for voltage regulator and parameters. These parameters are obtain through PSO search technique and are given in Table 4.1. Amplifier stage has first order delay element with gains and time constants of $K_{A}=200$ and $T_{A}=0.015$, respectively. Ideal actuator is considered for this case study with gain $K_{E}=1$ and time constant $T_{E}=0$. Backstepping control inputs are speed deviation $\Delta \omega$ and electric power deviation $\Delta P_{e}$. To implement control law (3.51), $\Delta \delta$ is also required. Integration of shaft speed $\Delta \omega$ is used to obtain $\Delta \delta$ with zero initial condition. 


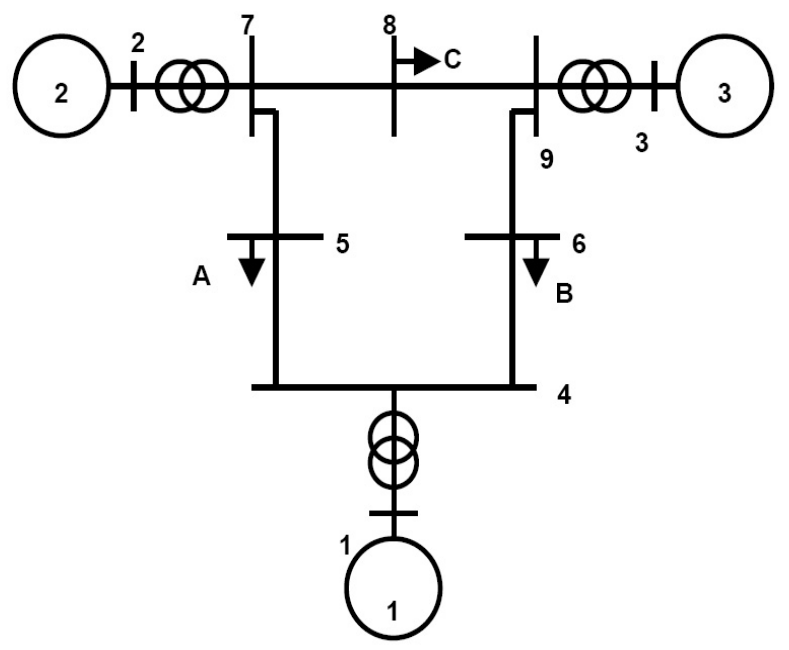

Figure 4.1: Three Machine Nine Bus Power System

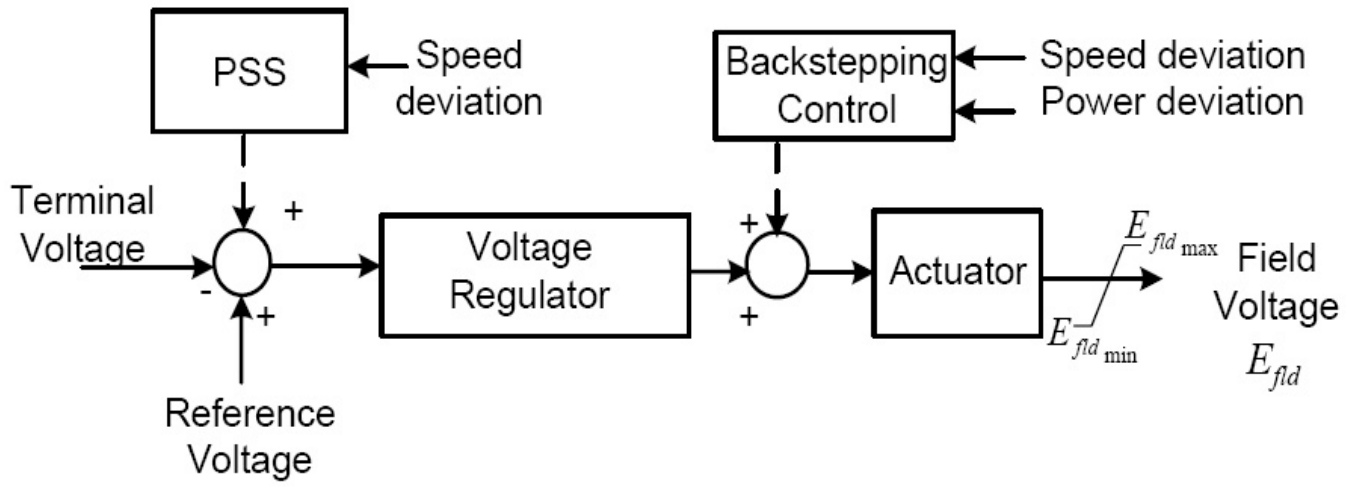

Figure 4.2: Excitation system control

Table 4.1: Parameters for Lead-Lag Stage $\left(T_{c}, T_{B}\right)$

\begin{tabular}{c|c|c}
\hline Generator & $T_{C}$ & $T_{B}$ \\
\hline 1 & 0.0675 & 0.3401 \\
\hline 2 & 0.2125 & 0.2687 \\
\hline 3 & 0.1269 & 0.1199 \\
\hline
\end{tabular}




\section{Scenario}

The proposed controller, based on (3.51), is implemented on generators 1,2 and 3. A three phase fault is applied on transmission line $6-9$ at $100 \mathrm{msec}$. The line is removed and fault is cleared at $180 \mathrm{msec}$. Following control strategies are analyzed:

- Backstepping controller with actuator (Figure 4.2), is implemented on each generator. Controller gains are tuned by PSO technique by optimizing the objective functions(4.1-4.3). Following gains $k_{1}, k_{2}$ are obtained through PSO search algorithm (Table 4.2). Parameters for wash-out stage and lead-lags for PSS are given in [9]

Table 4.2: Parameters for Backstepping Controller with Different Objective Functions

\begin{tabular}{c|c|c|c|c|c}
\hline$(I)$ & $k_{1}$ & $k_{2}$ & $(I I I)$ & $k_{1}$ & $k_{2}$ \\
\hline Gen\#1 & 2.2635 & 2.9467 & Gen\#1 & 1.753 & 2.9983 \\
\hline Gen\#2 & 2.5462 & 2.2950 & Gen\#2 & 2.3071 & 1.4592 \\
\hline Gen\#3 & 1.4030 & 1.8439 & Gen\#3 & 1.2654 & 1.006 \\
\hline$(I I)$ & $k_{1}$ & $k_{2}$ & $(I V)$ & $k_{1}$ & $k_{2}$ \\
\hline Gen\#1 & 2.3032 & 2.5140 & Gen\#1 & 1.5136 & 1.7463 \\
\hline Gen\#2 & 1.9657 & 2.990 & Gen\#2 & 2.4022 & 2.5833 \\
\hline Gen\#3 & 2.0906 & 2.70978 & Gen\#3 & 2.1806 & 2.7408 \\
\hline
\end{tabular}

Table 4.3: Parameters for PSS

\begin{tabular}{c|c|c|c|c|c|c}
\hline Generators & $K_{w}$ & $T_{w}$ & $T_{1}$ & $T_{2}$ & $T_{3}$ & $T_{4}$ \\
\hline Gen\#1-3 & 13.7750 & 1.410 & 0.154 & 0.033 & 0.154 & 0.033 \\
\hline
\end{tabular}

Figure 4.3 shows comparison between backstepping controllers tuned by objective functions (4.1-4.3). Relative rotor angles $\delta_{21}$ and $\delta_{31}$ indicates that the system finds new operating point that is different from pre-fault operating point. This happens because transmission line $6-9$ does not reclose afterward, and system configuration has changed. Also proposed controller can effectively damp generator shaft oscillations. Terminal voltages of generators 2,3 and control signals are shown in Figures 4.4 and 4.5 respectively. 

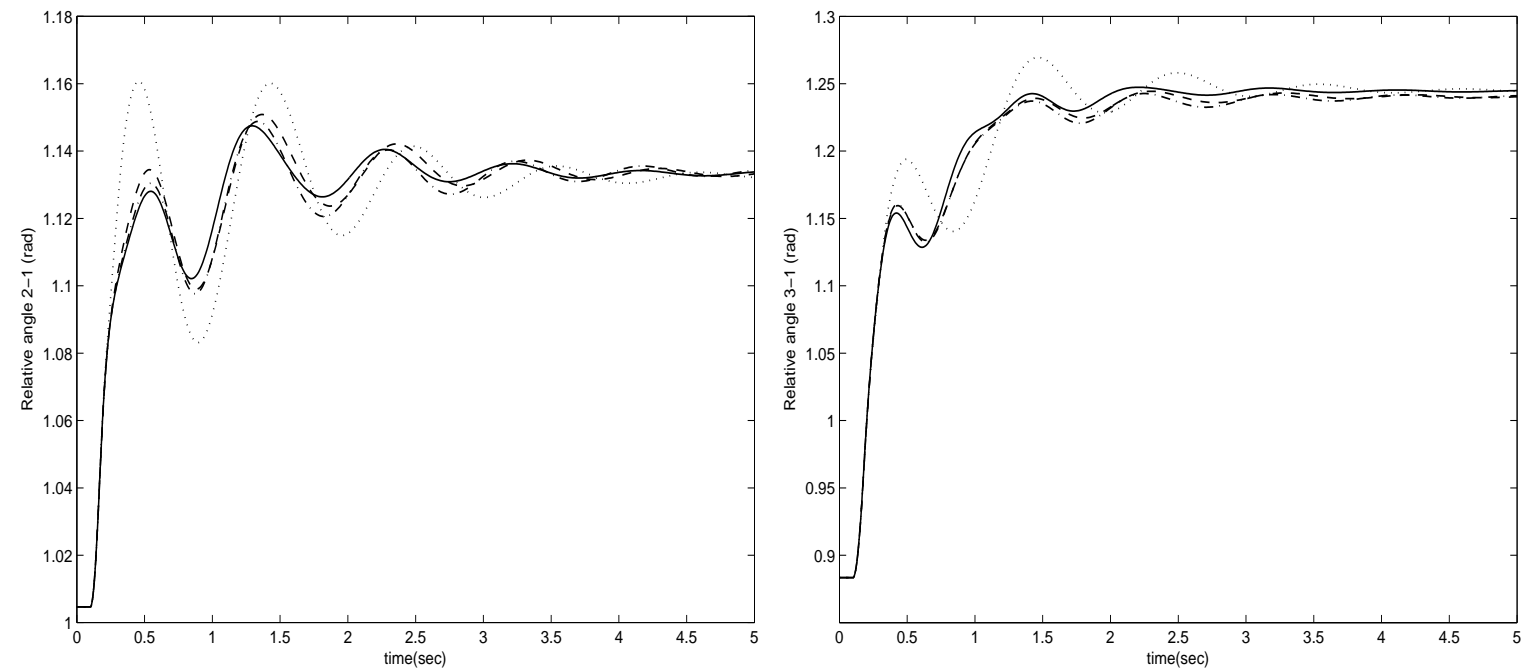

Figure 4.3: Relative rotor angles $2-1$ and $3-1$ (rad), exciter with backstepping damping controller with objective function $J(I)$ (solid line-), Objective function $J(I I)$ (dash line--), Objective function $J(I I I)$ (dash-dot line-.), PSS (dot line :)
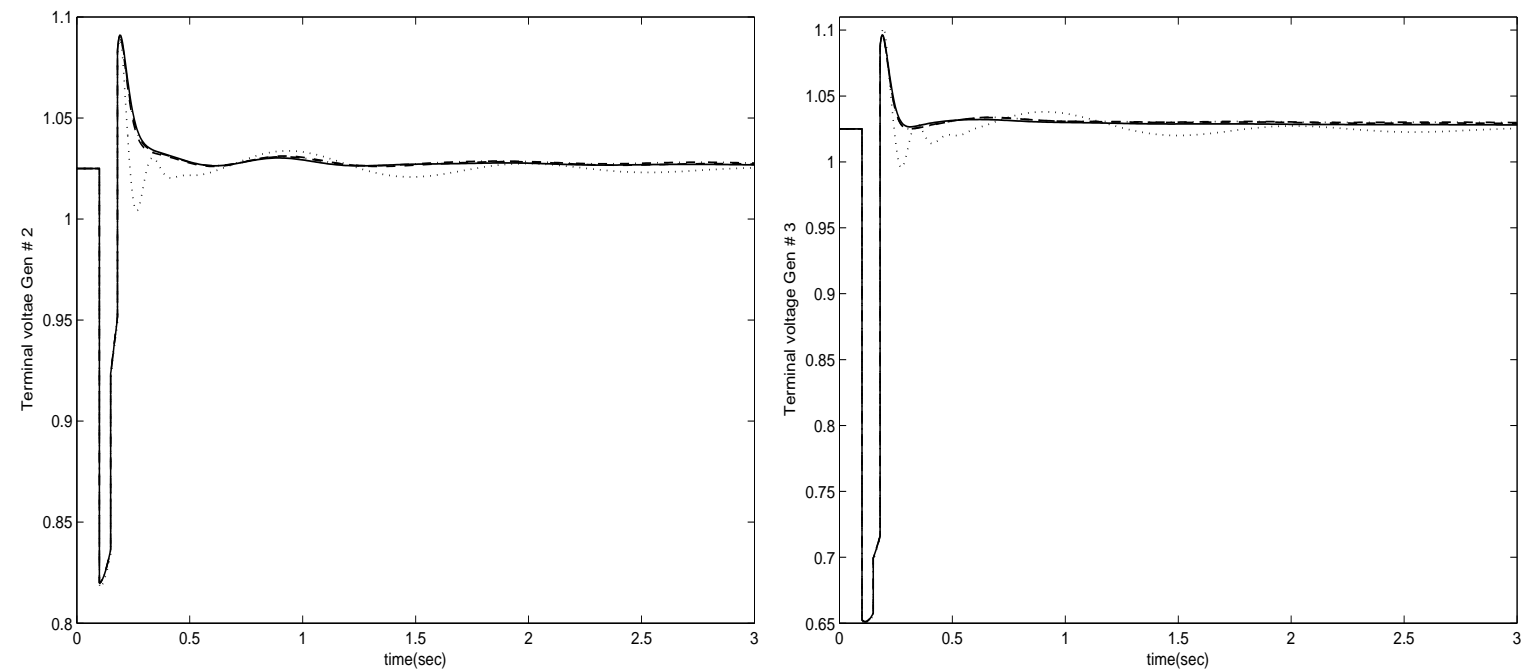

Figure 4.4: Terminal voltages for generators 2 and 3, exciter with backstepping damping controller with objective function $J(I)$ (solid line -), Objective function $J(I I)$ (dash line--), Objective function $J$ (III) (dash-dot line-.), PSS (dot line :)

An approach to design controls of generator excitation based on backstepping were presented in this subsection. The control parameters are tuned by a particle swarm optimization technique. The objective is to enhance stability of the power system and damp the oscillations during and after the contingencies. Based on numerical simulations, in this subsection, it is concluded that proposed controllers improves transient stability in 3 machine 9 bus test 
CHAPTER 4. TRANSIENT STABILITY ENHANCEMENT USING EXCITATION CONTROL47
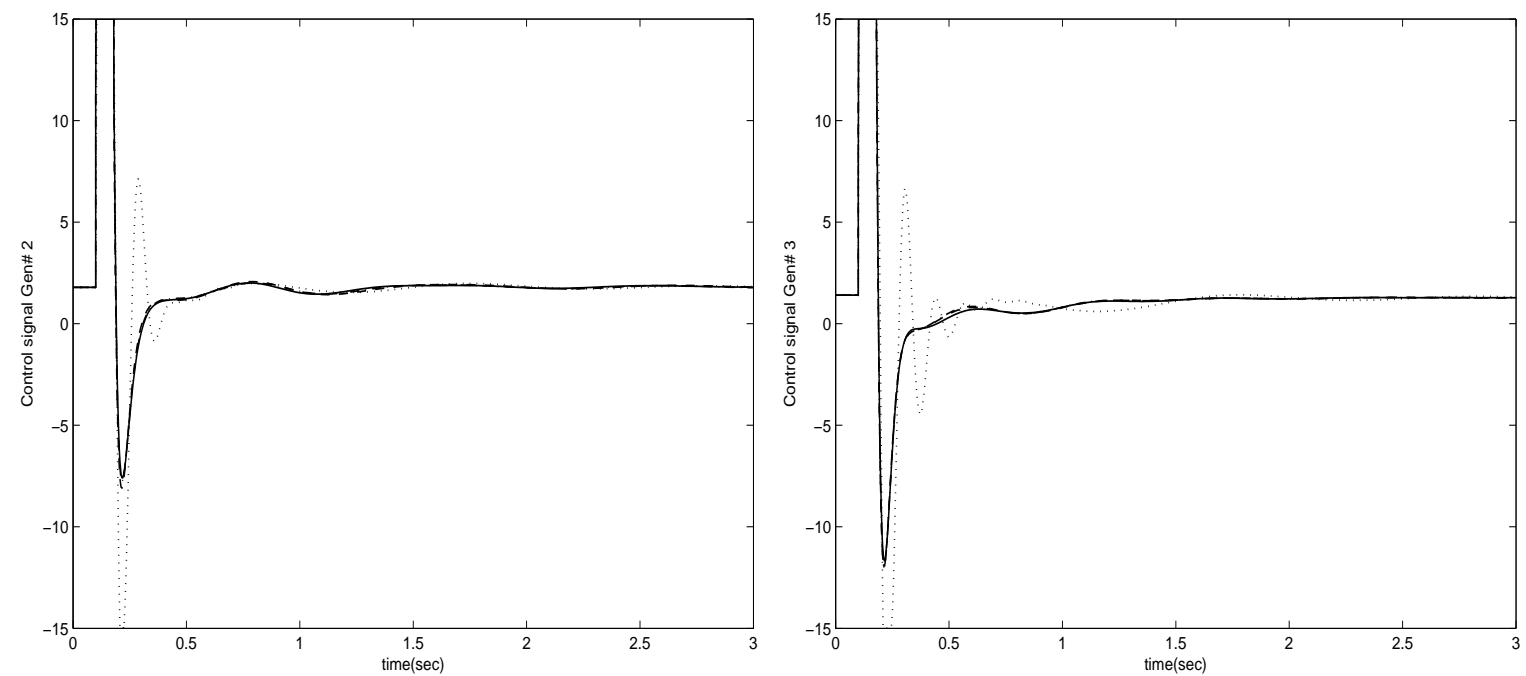

Figure 4.5: Control signals for generators 2 and 3, exciter with backstepping damping controller with objective function $J(I)$ (solid line -), Objective function $J(I I)$ (dash line --), Objective function $J(I I I)$ (dash-dot line -.), PSS (dot line :)

system.

It is assumed that interconnection terms with $I_{d i}$ and $I_{q i}$ and remote information is available measurements for each generator, in order to implement damping controller through excitation control of generator. To remove this assumption, two approaches will be considered in section 4.2 and 4.3 respectively. 


\subsection{Extended-Backstepping Control of Power Systems}

\subsubsection{Introduction}

In this section backstepping controller with additive nonlinear damping is applied for stability enhancement of multi-machine power systems. The decentralized control scheme is practical in systems especially when remote information are estimated locally. Remote information from one generator to other through transmission lines are modeled and considered as external disturbance through each plant. Controller has additive nonlinear damping to compensate the effect of this disturbance.

The organization of this section is as follows: In subsection 4.2.2 controller, extended-backstepping, is designed based on a recursive technique to obtain stabilizing control via Lyapunov function. Additive nonlinear damping (extended term) is added to backstepping controller and is used to counteract the effect of disturbance. Power system differential and algebraic equations are given in subsection 4.2.3. Subsection 4.2.4 explains interface modeling as external disturbance. Subsection 4.2.5 presents the optimal settings for extended-backstepping controller gains. Gain setting for the controller is obtained with Particle Swarm Optimization. Benchmark system with 50 machines 145 buses system presented in subsection 4.24 for implementing the proposed technique.

\subsubsection{Backstepping Control Design in Presence of Disturbance}

In this subsection a brief overview of backstepping control design for a nonlinear system with a disturbance is presented. To apply this method, the system is assumed to have a parametric strict-feedback form (3.6), which for a system with two states $\left(x, z_{1}\right)$ :

$$
\begin{aligned}
\dot{x} & =f_{0}(x)+g_{0}(x) z_{1} \\
\dot{z_{1}} & =f_{1}\left(x, z_{1}\right)+g_{1}\left(x, z_{1}\right)(u+d)
\end{aligned}
$$

The designed controller $u$ has two components. First component, $u_{B}$, is obtained using backstepping techniques while ignoring the disturbance $d$, and a second component, $u_{D}$, is an additional damping term added to counteract the effect of the disturbance. Hence, the final control has two parts $u=u_{B}+u_{D}$. 


\section{Backstepping Control}

The first component $u_{B}$ (backstepping controller) is designed as follows. The disturbance $d$ is neglected here.

Define virtual control $u_{B 1}$ :

$$
u_{B}=\frac{1}{g_{1}\left(x, z_{1}\right)}\left[u_{B 1}-f_{1}\left(x, z_{1}\right)\right]
$$

Hence, equation (4.6) can be written as:

$$
\dot{z}_{1}=u_{B 1}
$$

$z_{1}$ drives subsystem $x$ as seen from (4.5). It is composed of two components, one of which, $\phi_{0}(x)$, is to stabilize this subsystem, and a second part is to guarantee the stability of the overall system (see steps 1 and 2 below). Hence:

$$
z_{1}=\phi_{0}(x)+v
$$

The above equation is used to eliminate $z_{1}$. Taking the derivative with respect to time of (4.9) yields:

$$
\dot{z}_{1}=\dot{v}+\dot{\phi}_{0}(x)=u_{B 1}
$$

Equation (4.10) is re-arranged as:

$$
\dot{v}=\dot{z}_{1}-\dot{\phi}_{0}(x)=u_{B 1}-\dot{\phi}_{0}(x)
$$

Finally, the original system without disturbance is written as:

$$
\begin{aligned}
\dot{x} & =\left[f_{0}(x)+g_{0}(x) \phi_{0}(x)\right]+g_{0}(x) v \\
\dot{v} & =w
\end{aligned}
$$

where

$$
w=u_{B 1}-\dot{\phi}_{0}(x)
$$

Step 1: Let $W(x)>0$ be positive definite function. Find a Lyapunov function $V_{0}(x)$ (simple quadratic function) for the unforced system (4.11) and $\phi_{0}(x)$ such that

$$
\begin{aligned}
& \dot{V}_{0}(x)=\frac{\partial V_{0}}{\partial x}\left(f_{0}(x)+g_{0}(x) \phi_{0}(x)\right) \leq-W(x) \\
& \dot{V}_{0}(x)=x\left(f_{0}(x)+g_{0}(x) \phi_{0}(x)\right) \leq-W(x)
\end{aligned}
$$


In this case the unforced system is asymptotically stable.

Step 2: Find $w$ to stabilize (4.11), (4.12)

Let $V_{1}\left(x, z_{1}\right)=V_{0}(x)+\frac{1}{2} v^{2}$ be a Lyapunov function for (4.11) and (4.12). Its derivative along the state trajectory is given by:

$$
\begin{aligned}
\dot{V}_{1}\left(x, z_{1}\right) & =\dot{V}_{0}(x) \dot{x}+v \dot{v} \\
& =\frac{\partial V_{0}}{\partial x}\left(f_{0}(x)+g_{0} z_{1}\right)+v w \\
& =x\left(f_{0}(x)+g_{0}\left[\phi_{0}(x)+v\right]\right)+v w \\
& =x\left(f_{0}(x)+g_{0} \phi_{0}(x)\right)+x g_{0}(x) v+v w
\end{aligned}
$$

Substitution of (4.14) in (4.15) yields:

$$
\dot{V}_{1}\left(x, z_{1}\right) \leq-W(x)+x g_{0}(x) v+v w
$$

$w$ is chosen in a way to cancel the indefinite term and provide more negative definite to (4.16).

$$
w=-x g_{0}(x)-k_{1} v
$$

then

$$
\begin{array}{r}
\dot{V}_{1}\left(x, z_{1}\right) \leq-W(x)-k_{1} v^{2} \\
\dot{V}_{1}\left(x, z_{1}\right) \leq-W(x)-k_{1}\left(z_{1}-\phi_{0}(x)\right)^{2}
\end{array}
$$

Therefore, (4.18) results in negative definite if the control gain $k_{1}$ is positive.

$u_{B 1}$ is obtained from (4.13) (4.9) and (4.17). Finally, the backstepping controller $u_{B}$ is obtained by substituting $u_{B 1}$ in (4.7) as follow:

$$
\begin{array}{r}
u_{B 1}=w+\dot{\phi}_{0}(x)=-x g_{0}(x)-k_{1} v+\dot{\phi}_{0}(x) \\
u_{B}=\frac{1}{g_{1}}\left[-x g_{0}(x)-k_{1}\left[z_{1}-\phi_{0}(x)\right]+\dot{\phi}_{0}(x)-f_{1}\right]
\end{array}
$$

\section{Additive Nonlinear Damping}

Now, including disturbance $d$ to the local system, nonlinear damping term $u_{D}$, is added using the results provided in the following lemma [64],[49]

Lemma: Consider the system $(4.5,4.6)$, The following control

$$
u=u_{B}-\gamma \frac{\partial V_{1}\left(x, z_{1}\right)}{\partial z_{1}} g_{1}\left(x, z_{1}\right), \gamma>0
$$


where $u_{B}$ is given in (4.19), guarantees global uniform boundedness of $X=\left(x, z_{1}\right)$ and convergence to the residual set

$$
\Re=\left\{X:|X| \leq \varsigma_{1}^{-1} \circ \varsigma_{2} \circ \varsigma_{3}^{-1}\left(\frac{\|d\|_{\infty}^{2}}{4 \gamma}\right)\right\}
$$

where $\varsigma_{1}, \varsigma_{2}$, and $\varsigma_{3}$ are called $\kappa_{\infty}$ function such that

$$
\begin{aligned}
\varsigma_{1}(|X|) \leq V(X) & \leq \varsigma_{2}(|X|) \\
\varsigma_{3}(|X|) & \leq W(X)
\end{aligned}
$$

Hence:

$$
u_{D}=-\gamma \frac{\partial V_{1}\left(x, z_{1}\right)}{\partial z_{1}} g_{1}\left(x, z_{1}\right)
$$

Proof of lemma is given in appendix F. A continuous function $\varsigma_{1}$ is said to belong to $\kappa_{\infty}$ if

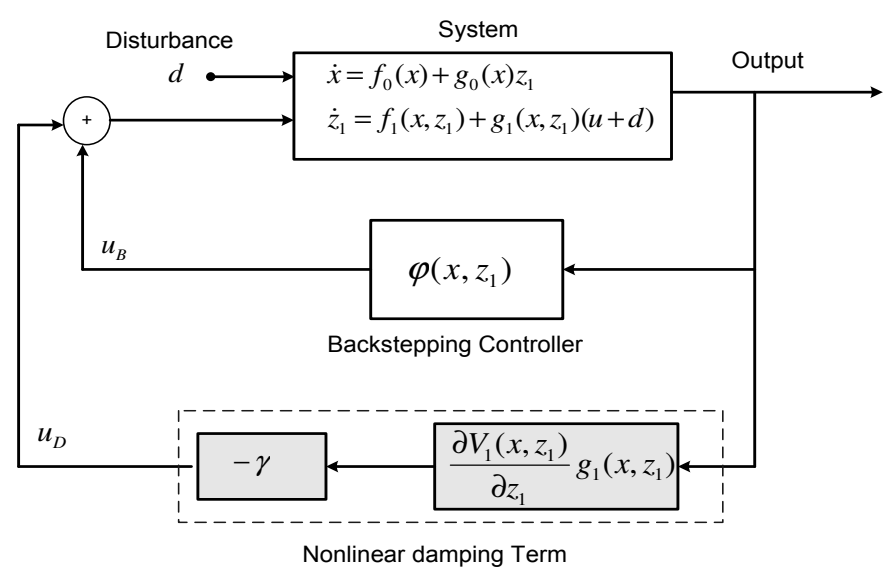

Figure 4.6: Backstepping Control and Nonlinear Damping

it is strictly increasing, $\varsigma_{1}(r) \rightarrow \infty$ as $r \rightarrow \infty$ and $\varsigma_{1}(0)=0$. Once $\varsigma_{1}$ is a class $\kappa_{\infty}$ then the inverse $\varsigma_{1}^{-1}$ belongs to the same class. Also $\varsigma_{1}^{-1} \circ \varsigma_{2} \circ \varsigma_{3}^{-1}(\cdot)$ is composition of $\varsigma_{1}, \varsigma_{2}$, and $\varsigma_{3}$ and defined as $\varsigma_{1}^{-1} \circ \varsigma_{2} \circ \varsigma_{3}^{-1}=\varsigma_{1}^{-1}\left(\varsigma_{2}\left(\varsigma_{3}^{-1}(\cdot)\right)\right)[60]$.

$k_{1}, \gamma$ are controller and nonlinear damping gains respectively. A large $\gamma$ results in small disturbance, i.e. $\|d\|_{\infty}<<1$ and will guarantee stability of the system. Hence, $\gamma$ is a 
design parameter. Large $\gamma$ will however saturate the controller signal. Therefore, it will put some restriction on the size of the disturbance. A schematic block diagram of the extendedbackstepping is shown in Figure 4.6.

\subsubsection{Disturbance Modeling}

To apply the extended-backstepping method as described in subsection 4.2.2 to power systems, the system has to be in strict feedback form. The power system is an interconnection of many generating power plants to load centers through transmission lines. To design decentralized controllers, each generator is modeled as an independent subsystem in a strict feedback form, and the effect of the external system is in a form of a disturbance, consistent with the modeling presented previously $(3.46,4.33,3.48)$. Hence, each generator is modeled as:

$$
\begin{aligned}
\dot{x} & =f_{0}(x)+g_{0}(x) z_{1} \\
\dot{z}_{1} & =f_{1}\left(x, z_{1}\right)+g_{1}\left(x, z_{1}\right) z_{2} \\
\dot{z}_{2} & =f_{2}\left(x, z_{1}, z_{2}\right)+g_{2}\left(x, z_{1}, z_{2}\right)(u+d) .
\end{aligned}
$$

where

$$
\begin{aligned}
& x=\Delta \delta \quad f_{0}=0 \quad g_{0}=1 \\
& z_{1}=\Delta \omega f_{1}=-\frac{D}{2 H} z_{1} \quad g_{1}=-\frac{\omega_{0}}{2 H} \\
& z_{2}=\Delta P_{e} \quad f_{2}=-\frac{\Delta P_{e}}{T_{q o}^{\prime}} \quad g_{2}=\frac{I_{q}}{T_{d o}^{\prime}} \\
& u=\Delta E_{f l d}
\end{aligned}
$$

and $d^{\prime}=g_{2}\left(x, z_{1}, z_{2}\right) d$ is

$$
\begin{aligned}
d^{\prime} & =-\frac{I_{q} I_{d}\left(X_{q}-X_{q}^{\prime}\right)}{T_{q o}^{\prime}}+E_{q}^{\prime} I_{q}\left(\frac{1}{T_{q o}^{\prime}}-\frac{1}{T_{d o}^{\prime}}\right)-\frac{P_{e}^{\circ}}{T_{q o}^{\prime}}+ \\
& +E_{d}^{\prime} \dot{I}_{d}+E_{q}^{\prime} \dot{I}_{q}+\frac{I_{q} I_{d}\left(X_{d}-X_{d}^{\prime}\right)}{T_{d o}^{\prime}}+\frac{E_{f l d}^{\circ} I_{q}}{T_{d o}^{\prime}}
\end{aligned}
$$

Parameters and variables for synchronous generators and transmission lines are given in Table 3.1. Following the extended-backstepping technique in subsection 4.2 .2 , the final controller is given by

$$
u=\phi_{2}\left(x, z_{1}, z_{2}\right)-\gamma \frac{\partial V_{2}\left(x, z_{1}, z_{2}\right)}{\partial z_{2}} g_{2}\left(x, z_{1}, z_{2}\right)
$$

where $\phi_{2}\left(x, z_{1}, z_{2}\right), V_{2}\left(x, z_{1}, z_{2}\right)$ are obtained from (3.30) and (3.28), respectively. Extendedbackstepping control has the ability to work under diverse operating conditions and damp 
the oscillations effectively under small and large disturbances. Note that here $f_{2}$ term can be computed locally and does not include remote information.

\subsubsection{Overall System View}

A complete centralized control scheme in electric power system has excessive computation time, distortion in information transfer, and high cost. More feasible structure is obtained by decentralized scheme, where controllers only utilize local information and operate without the need for remote signals. To design decentralized controllers, each generator is modeled as an independent subsystem in a strict feedback form. The effect of the external system is considered as disturbances to the generator which is in concern. Extended-backstepping controller (4.27) is designed to stabilize each generator and to counteract the effect of external disturbances.

Note that based on aforementioned lemma (Appendix F), stability of n-machine power system, is guaranteed when a positive definite candidate Luapunov function

$$
V_{\text {total }}=\sum_{i=1}^{n}\left(V_{0}\left(x_{i}\right)+\frac{1}{2}\left[z_{1 i}-\phi_{0 i}\left(x_{i}\right)\right]^{2}+\frac{1}{2}\left[z_{2 i}-\phi_{1 i}\left(x_{i}, z_{1 i}\right)\right]^{2}\right)
$$

and has a negative definite derivative along state trajectories. Negative value of the derivative

$\dot{V}_{\text {total }}$ means that Lyapunov functions $\dot{V}_{\text {total }}$ decreases with time and tends toward it minimum value.

$$
\dot{V}_{\text {total }} \leq \sum_{i=1}^{n}-W_{i}\left(x_{i}\right)-k_{1 i}\left(z_{1 i}-\phi_{0 i}\left(x_{i}\right)\right)^{2}-k_{2 i}\left(z_{2 i}-\phi_{1 i}\left(x_{i}, z_{1 i}\right)\right)^{2}+\frac{1}{4 \gamma_{i}}\left\|d_{i}\right\|_{\infty}^{2}
$$

This condition is satisfied as long as

$$
\sum_{i=1}^{n}-W_{i}\left(x_{i}\right)-k_{1 i}\left(z_{1 i}-\phi_{0 i}\left(x_{i}\right)\right)^{2}-k_{2 i}\left(z_{2 i}-\phi_{1 i}\left(x_{i}, z_{1 i}\right)\right)^{2}+\frac{1}{4 \gamma_{i}}\left\|d_{i}\right\|_{\infty}^{2} \leq 0
$$

The effect of control gains and nonlinear damping factor somehow provide a balance to obtain the inequality condition of Lypunov stability and minimize the effect of disturbance. 


\subsubsection{Optimal Settings for Controller Gains}

Particle Swarm Optimization technique is used in this subsection to obtain optimal regions for controller gains. Performance index relating to optimization for control gains is defined as

$$
J_{\text {controller }_{i}}=\int_{0}^{t}\left[\alpha\left(\Delta \omega_{i}\right)^{2}+\beta\left(\Delta \delta_{i j}\right)^{2}+\lambda\left(\Delta E_{f l d_{i}}\right)^{2}\right] d t
$$

Control constraints

$$
\begin{aligned}
k_{i 1}^{\min }<k_{i 1} & <k_{i 1}^{\max } \\
k_{i 2}^{\min } & <k_{i 2}<k_{i 2}^{\max } \\
\Delta E_{f l d_{i}}^{\min } & <\Delta E_{f l d_{i}}<\Delta E_{f l d_{i}}^{\max }
\end{aligned}
$$

System constraints

$$
\begin{aligned}
\Delta \omega_{i}^{\min }<\Delta \omega_{i} & <\Delta \omega_{i}^{\max } \\
\Delta \delta_{i j}^{\min }<\Delta \delta_{i j} & <\Delta \delta_{i j}^{\max } \\
\Delta V_{\text {gen }_{i}}^{\min }<\Delta V_{\text {gen }_{i}} & <\Delta V_{\text {gen }_{i}}^{\max } .
\end{aligned}
$$

Table 4.4 shows parameters for controller performance index.

Table 4.4: Performance Index Parameters for Controller

\begin{tabular}{rll}
\hline$k_{i}$ & $\ldots$ & Controller's gains \\
$\Delta \omega_{i}$ & $\ldots$ & Speed deviation \\
$\Delta \delta_{i j}$ & $\ldots$ & Relative rotor angle deviation \\
$\Delta V_{g e n_{i}}$ & $\ldots$ & Terminal Voltage deviation \\
$\Delta E_{f l d i}$ & $\ldots$ & Control signal deviation from initial values \\
$\alpha, \beta, \lambda$ & $\ldots$ & Weighting factors \\
\hline
\end{tabular}

\subsubsection{Case Study}

\section{Fifty Machine System}

Mid-sized benchmark that retains the dynamic behavior of large scale power system is considered for implementing the proposed controller design. It consists of 50 generators, 44 are 
classical, and 6 are transient models [31]. Complete data related to generators and exciter data are given in Appendix D. Four nonlinear controllers are implemented on generators at related buses 93,104,110, and 111 [31]. Schematic block diagram for 50 machine power system is shown in Figure 4.7. Control gains are tuned with PSO algorithm and considered the same

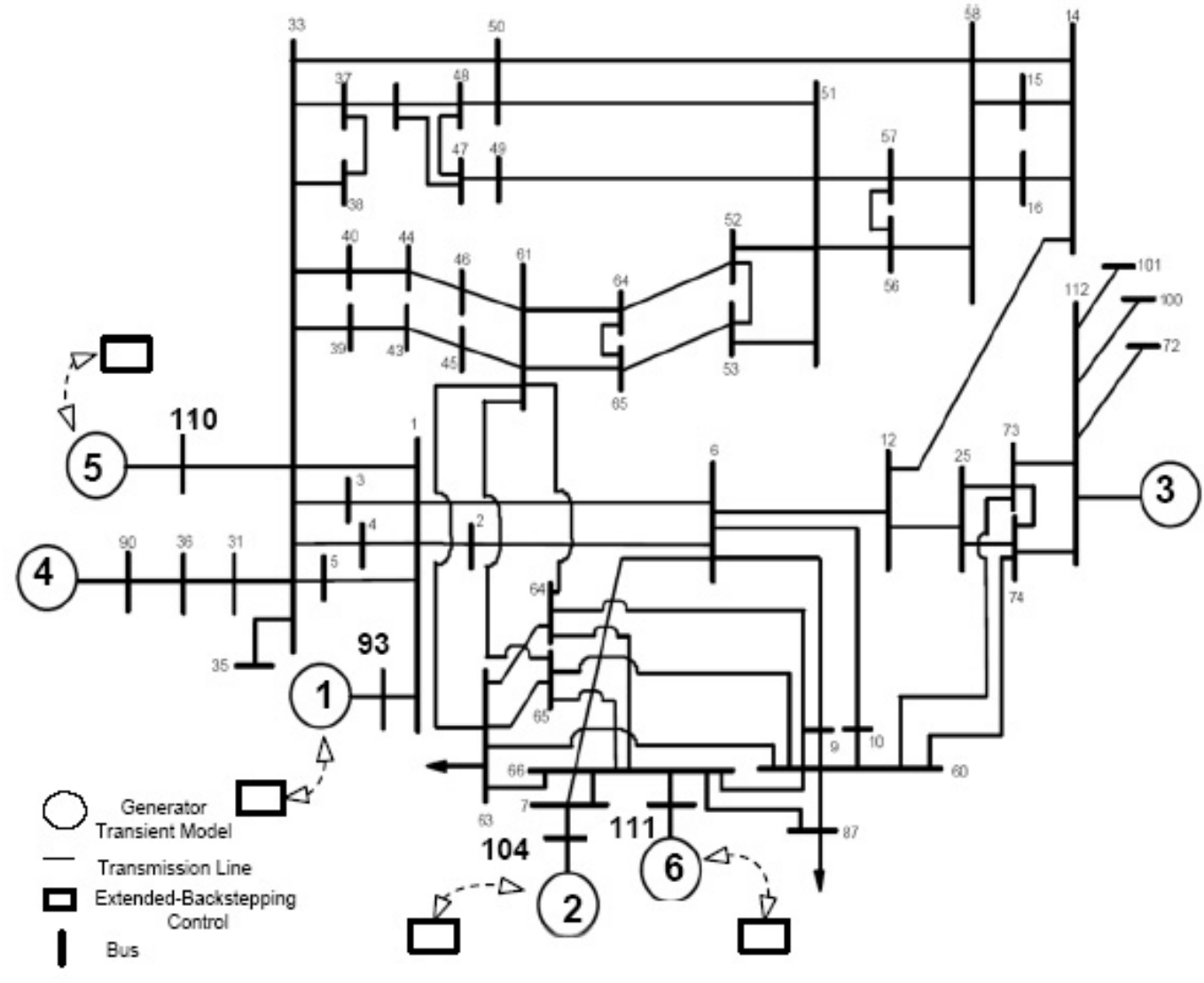

Figure 4.7: Fifty Machine System Single Line Diagram

for all scenarios.

\section{Excitation System Control Design}

Extended-backstepping control has ability to work under diverse operating points of the system, and as will be shown in case studies this nonlinear control works effectively under small and large disturbances. Figure 4.8 shows schematic block diagram of supplementary damping 
signal with exciter. Damping control signal $u$ is applied through the exciter. Comparison is held between the proposed damping controller and the PSS. It consists of amplifier gain $K_{A}$, amplifier time constant $T_{A}$, and saturation hard limit. The actuator is ideal with $K_{E}=1$ and $T_{E}=0$. In power system stability and control, the main idea of damping control is to suppress generator rotor oscillations under influence of contingencies and improve dynamic performance. Conventional damping control, power system stabilizer (PSS), is design based on linearized model of power system around small region of operating points. Comparison between extended-backstepping control and PSS is shown in following scenarios.

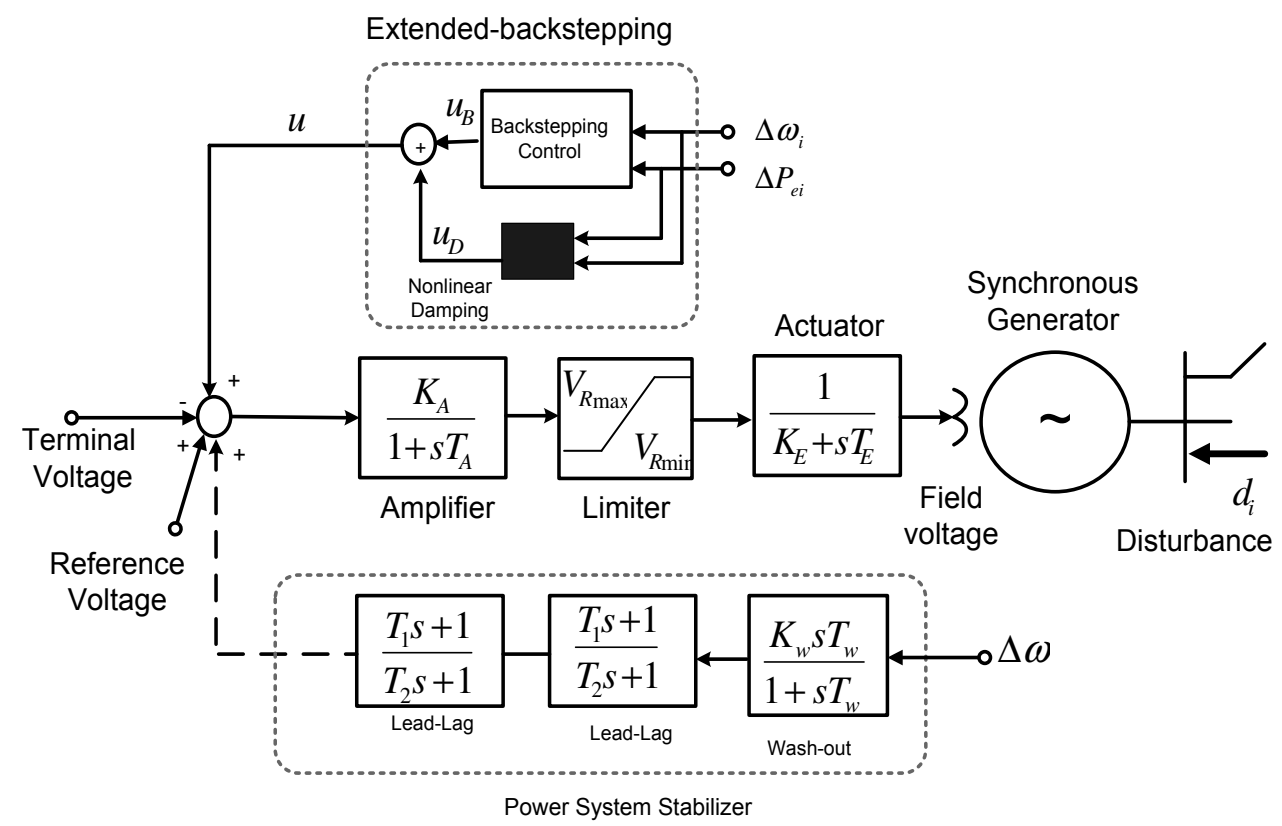

Figure 4.8: Supplementary Damping Controllers with Exciter

\section{Scenario-I Three Phase Fault}

For this scenario three phase fault is applied on transmission line $59-107$ at $100 \mathrm{msec}$, the fault is cleared at $300 \mathrm{msec}$. Controllers will sustain the stability of overall system even though the type and location of fault is altered. Same parameters (Table 4.5) are used for extendedbackstepping controller gains. Comparison is done between extended-backstepping control 
and power system stabilizer. PSS has two similar lead-lag with a wash-out stage (3.53). Two algorithms are used for tuning PSS gains. First, PSO is used to obtain PSS gains (Table 4.6). Second technique, Genetic Algorithm (GA), the gains are obtained from survey on tuning PSS gains [36]. The parameters for the excitation system are given in Appendix D. In Figure 4.9 extended-backstepping control with damping factor $\gamma=150$ (solid line) damp the rotor oscillations effectively when compared with PSS tuned by PSO (dash-dot line), PSS tuned by GA (dash line), and simple exciter with no damping controller (dot line). Controller signals for generators 2, 6 are shown in Figure 4.10. Larger control effort signals is used by the proposed controller to damp oscillations, since nonlinear damping term is introduced for this new control. Hence, there is a trade-off in balance between saturation of control signals and magnitude of nonlinear damping signal.

Table 4.5: Parameters for Backstepping control

\begin{tabular}{c|c|c|c}
\hline Generator \# & Bus \# & Gain $k_{1}$ & Gain $k_{2}$ \\
\hline 1 & 93 & 272.7240 & 63.6678 \\
\hline 2 & 104 & 251.1236 & 211.4062 \\
\hline 5 & 110 & 180.3733 & 240.5763 \\
\hline 6 & 111 & 277.0844 & 355.0586 \\
\hline
\end{tabular}

Table 4.6: Parameters for PSS tuned by PSO

\begin{tabular}{c|c|c|c|c|c}
\hline Generator \# & Bus \# & $K_{w}$ & $T_{w}$ & $T_{1}$ & $T_{2}$ \\
\hline 1 & 93 & 92.4223 & 3.4140 & 0.59 & 0.39 \\
\hline 2 & 104 & 28.3032 & 1.6609 & 1.01 & 0.35 \\
\hline 5 & 110 & 81.3726 & 8.5178 & 0.38 & 0.28 \\
\hline 6 & 111 & 7.1390 & 0.5353 & 0.99 & 0.40 \\
\hline
\end{tabular}

\section{Scenario-II Effect of Nonlinear Damping}

Three-phase to ground fault is applied on transmission line 61-63. Fault duration is 100 msec. The transmission line is removed at $200 \mathrm{msec}$ but not reclosed afterward. In this scenario the objective is to demonstrate the effect of nonlinear damping term to suppress the oscillations. 

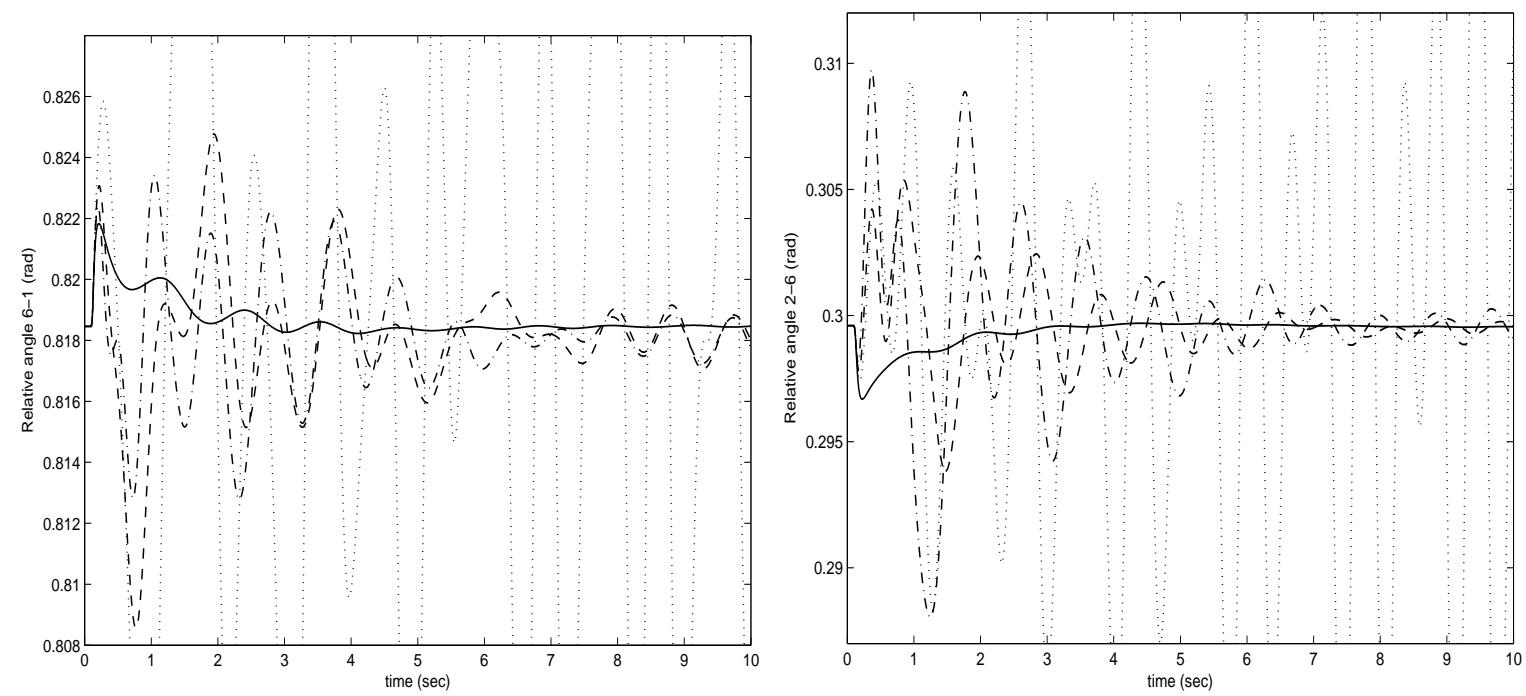

Figure 4.9: Relative rotor angles (rad) 6-1,6-3. Exciter with PSO-tuned extended-backstepping (solid line -), simple exciter with PSO-tuned PSS (dash-dot line-. ), simple exciter with GA-tuned PSS (dash line -- ), simple exciter without damping control (dot line .)
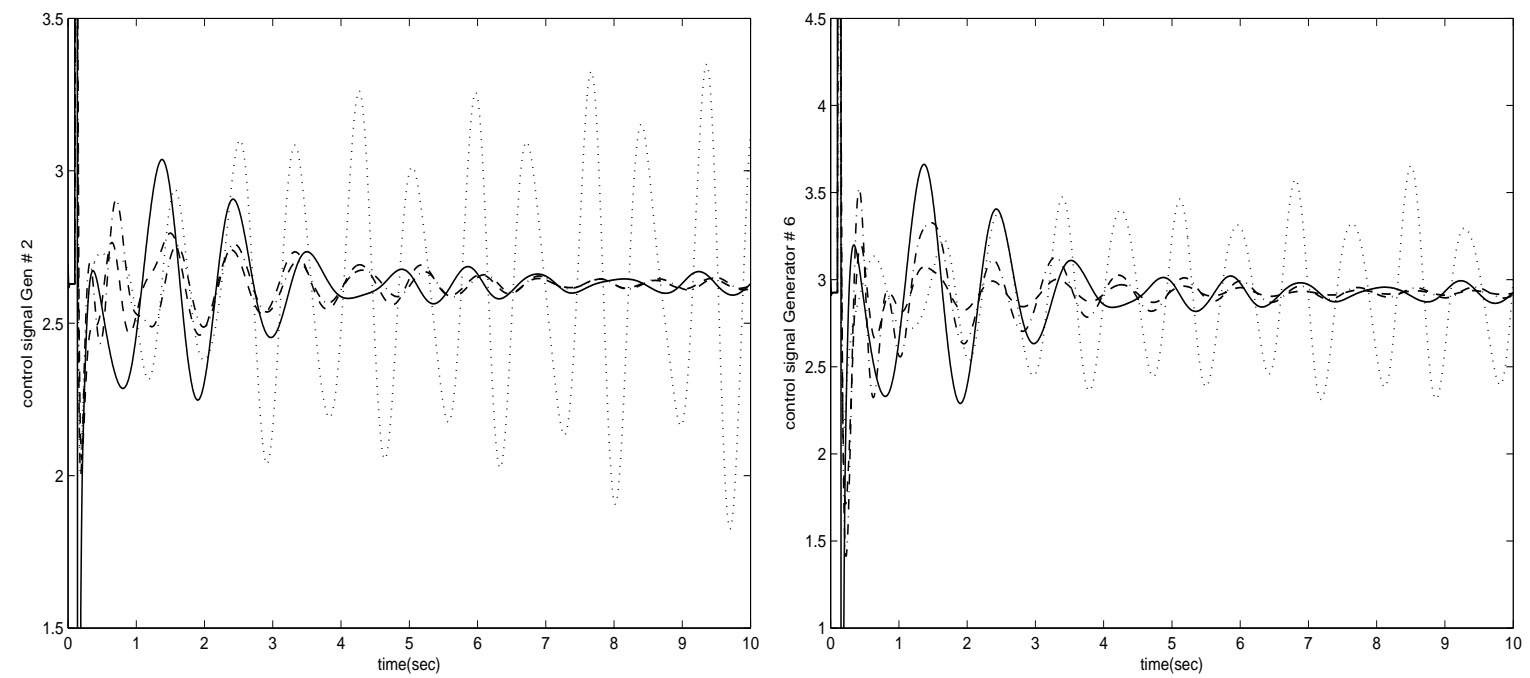

Figure 4.10: Excitation Control signals for Generators 2 and 6. Exciter with PSO-tuned extendedbackstepping (solid line -), simple exciter with PSO-tuned PSS (dash-dot line -. ), simple exciter with GA-tuned PSS (dash line -- ), simple exciter without damping control (dot line .) 
Figure 4.11 shows the effect of nonlinear damping factor $\gamma$, on speed deviation of generator 1 from nominal value. By increasing the gain $\gamma$ the system obtains additional damping and the oscillations are suppressed. Note that for very large value of $\gamma$ control signal will be saturated on upper and lower limits. The effect of saturation for generator 1 is also shown in Figure 4.12. In this section, coupling among generators through transmission lines is considered as external disturbance. Controllers were designed based on backstepping technique to stabilize the system, plus additive nonlinear damping were added to controller to counteract the effect of disturbance.

In selecting controller gains, emphasis was placed on damping the oscillations of rotor angle,
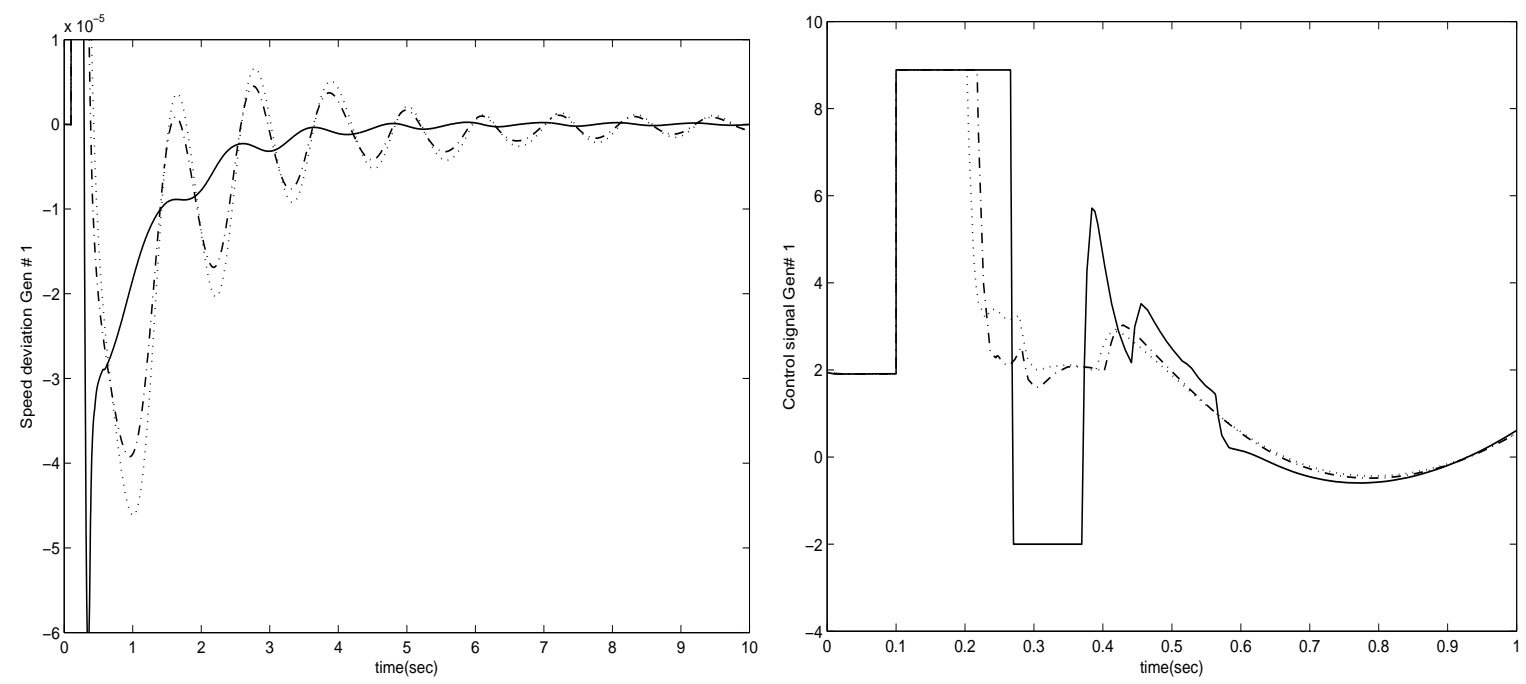

Figure 4.11: Effect of nonliear damping speed deviation and control signal for generator $1, \gamma=1500$ (solid line -), $\gamma=100$ (dash-dot line .), $\gamma=15$ (dot line.)

speed, power and terminal voltages from their nominal. The latter were achieved by PSO tuning technique.

\section{Effect of Noise}

Consider Scenario-II with reclosing the line at 250 msec. For this case Gaussian noise is included in disturbance term. The latter change makes the interface term a more realistic condition. Also the objective function has local information that includes speed deviations 

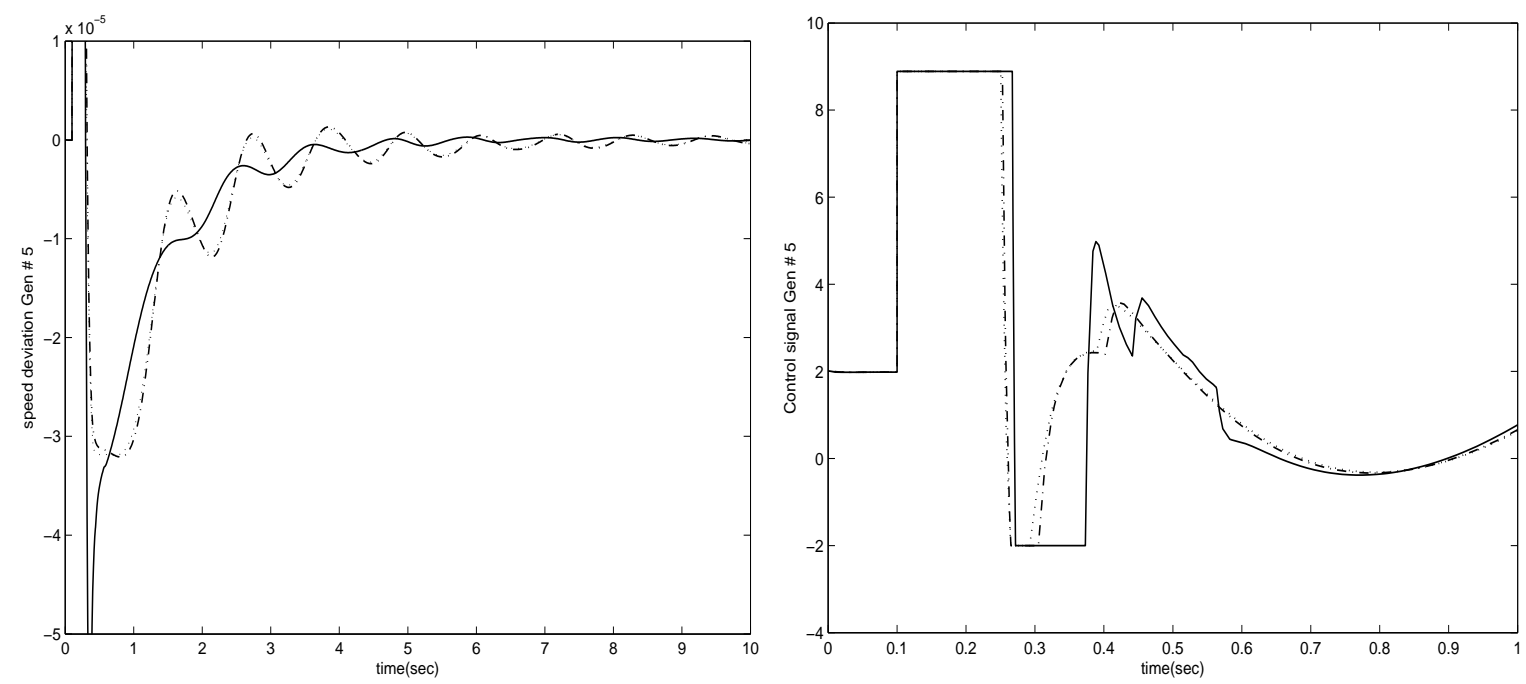

Figure 4.12: Effect of nonlinear damping speed deviation and control signal for generator $5, \gamma=1500$ (solid line -), $\gamma=100$ (dash-dot line .), $\gamma=15$ (dot line.)

from generators.

$$
J_{\text {controller }_{i}}=\int_{0}^{t} \alpha\left(\Delta \omega_{i}\right)^{2} d t
$$

Backstepping controller gains are given in Table 4.7. Effect of control signals for generators

Table 4.7: Parameters for Backstepping control

\begin{tabular}{c|c|c|c}
\hline Generator \# & Bus \# & Gain $k_{1}$ & Gain $k_{2}$ \\
\hline 1 & 93 & 189.8 & 16.21 \\
\hline 2 & 104 & 151.1 & 370.5 \\
\hline 5 & 110 & 400 & 395.5 \\
\hline 6 & 111 & 246.4 & 0.14 \\
\hline
\end{tabular}

1, 2, 5 and 6 with $\gamma=1000$ are shown in 4.13. Sudden jumps in control signals are caused by the disturbances. The effect of nonlinear damping $\gamma$ is investigated on suppressing the oscillations of generator. Figures 4.13 show that by increasing $\gamma$, the oscillations will damp faster. Gaussian noise is included in disturbance $d$. The noise has zero mean, fixed variance of 0.2 and sample time 1 second that has direct effects on actuator signal. Nonlinear damping added to backstepping controller can suppress the oscillations in presence of noisy measurement. 

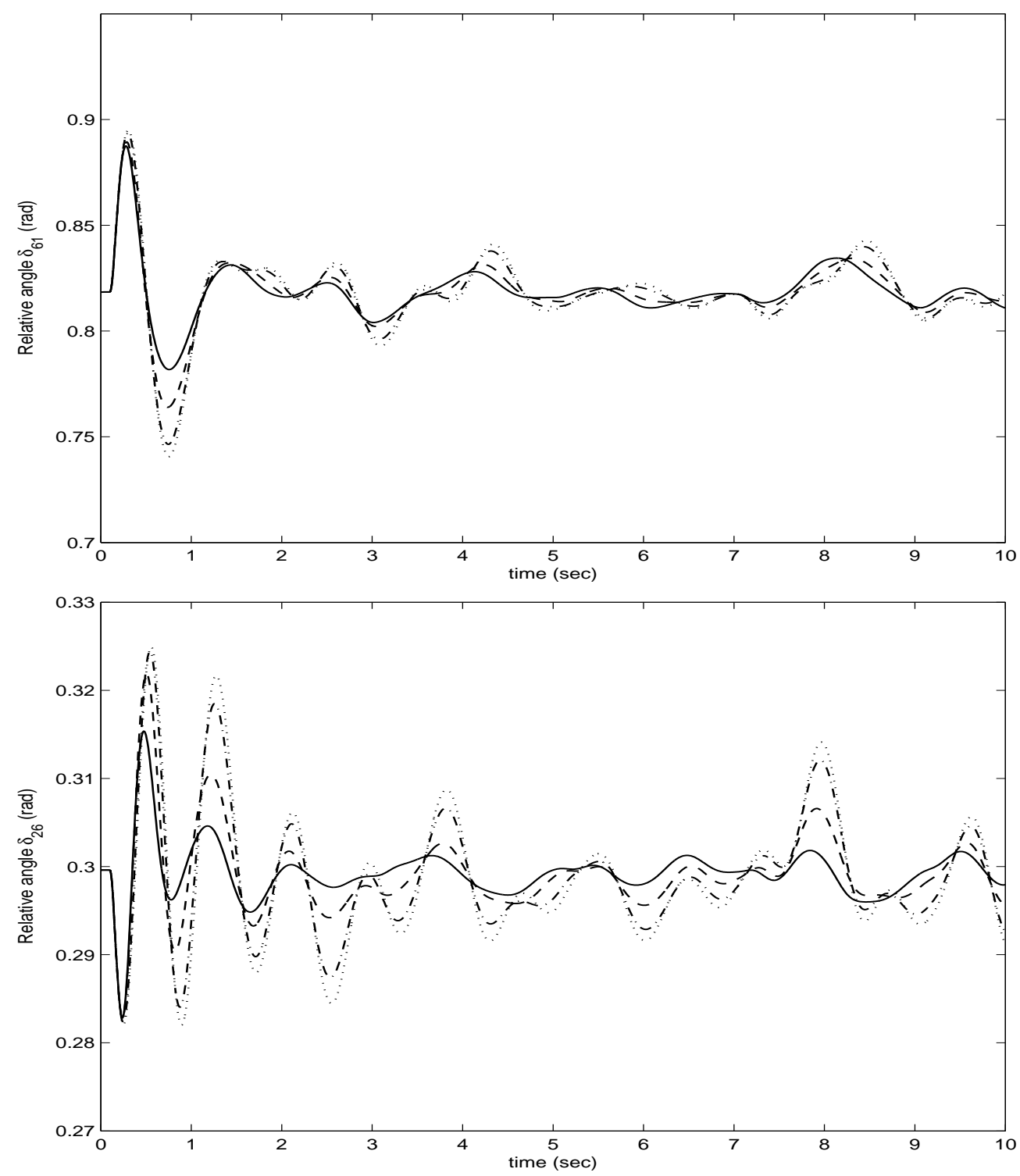

Figure 4.13: Relative rotor angles (rad) 6-1,2-6. Simple exciter with extended backstepping control with $\gamma=1000$ (solid line -), $\gamma=400$ (dash line --), $\gamma=90$ (dahs-dot line -.), $\gamma=20$ (dot line :) 
Backstepping technique that presented here can solve stabilization problems under conditions less restrictive that those encountered in other methods, since it exploits the flexibility assured by lower-order and scalar systems. Moreover, backstepping exhibits its full power in the presence of uncertain nonlinearities such as interface between generators. In summary In this section backstepping design and nonlinear damping have been proposed as a new approach for controlling a generator dynamics. Each generator is modeled as a subsystem connected to the grid. The effect of interconnection on each subsystem is considered as external disturbance. Backstepping controller and nonlinear damping are provided to stabilize the generator and counteract the effect of disturbance respectively. 


\subsection{PSO-tuned Adaptive Backstepping Control}

\subsubsection{Introduction}

In this section, the approach toward decentralized control is based on adaptive backstepping. The adaptation laws are used to estimate the effects of the rest of the system on concerned generator, which allows for a decentralized control design. The section is organized as follows. In Subsection 4.3.2 a strict feedback generator model suitable for decentralized control design is presented. In Subsection 4.3.3 the adaptive backstepping control design algorithm is presented. Interface variables are estimated with linear estimator whose coefficients are adapted. The control and adaptation gains are obtained using a Particle Swarm Optimization (PSO) search technique in Section 4.3.4. Case study is provided in Subsection 4.3.5 to illustrate the effectiveness of the proposed controller.

\subsubsection{Problem Formulation}

To apply the design technique proposed in this section, the generator model is (1) cast in a strict feedback form [64], and (2) each machine is modeled as an independent dynamic subsystem. The starting point is the transient two-axis generator model given in section 3.1.3. To obtain the strict feedback form model, acceleration power instead of direct and quadrature voltages is used as a state variable. The decoupling of the generator from the rest of the system is obtained by considering the effect of the rest of the system on each generator as a disturbance. Therefore, each generator is modeled by the following state equations.

$$
\begin{aligned}
\Delta \dot{\delta}_{i} & =\Delta \omega_{i} \\
\Delta \dot{\omega}_{i} & =-\frac{D_{i}}{2 H_{i}} \Delta \omega_{i}-\frac{\omega_{i o}}{2 H_{i}} \Delta P_{e_{i}} \\
\Delta \dot{P}_{e_{i}} & =-\Delta P_{e_{i}} \frac{1}{T_{q o i}^{\prime}}+\beta_{i} \Delta E_{f l d_{i}}+d_{i}^{\prime}
\end{aligned}
$$

where the coupling term $d_{i}^{\prime}$ is given by equation (4.26). This term includes local and remote information. Here, it is expressed as a linear uncertain function from two local measurements with parameters that will be estimated. Here, uncertain function is considered as first order differential equation,

$$
d_{i}^{\prime} \approx \theta_{1 i} \Delta P_{e_{i}}+\theta_{2 i} \Delta \dot{P}_{e_{i}}
$$


where $\theta_{1 i}$ and $\theta_{2 i}$ from 4.34) are uncertain values which has to be estimated through adaptation law. In generic terms, the equation set (4.33) for $i^{\text {th }}$ generator is:

$$
\begin{aligned}
& \dot{x}_{1}=b_{1} x_{2} \\
& \dot{x}_{2}=b_{2} x_{3}+b_{3} x_{2} \\
& \dot{x}_{3}=\beta u+b_{4} x_{3}+d^{\prime}
\end{aligned}
$$

where, disturbance $d^{\prime}$ is chosen as (4.34). State variables are $x_{1}=\Delta \delta \quad x_{2}=\Delta \omega \quad x_{3}=\Delta P_{e}$.

Parameters are $b_{1}=1 \quad b_{2}=-\frac{\omega_{0}}{2 H} \quad b_{3}=-\frac{D}{2 H} \quad b_{4}=-\frac{1}{T_{q o i}^{\prime}}$, and control input $u=\Delta E_{f l d}$ appears in the last equation. $\beta, \theta_{1}, \theta_{2}$ are uncertainties, not known a priori.

\subsubsection{Adaptive Backstepping Control Design}

The objective is to stabilize the system (4.35-4.37) using backstepping control which is to steer $x_{1}$ to its desired value $x_{1}^{d}=\alpha_{0}=$ constant, then find $x_{2}$ to stabilize (4.35), and $x_{3}$ to stabilize (4.36) and finally $u$ to stabilize (4.37) and hence the overall system. Consequently once the control signal $u$ is obtained, it can stabilize dynamic of $x_{3}$. Backstep to equation (4.36), $x_{3}$ controls the dynamic of $x_{2}$. Again backstep to equation (4.35), $x_{2}$ grasps dynamic of $x_{1}$. Hence the overall system is stabilized. Each of the states $x_{2}$ and $x_{3}$ will have virtual trajectories $\alpha_{1}$ and $\alpha_{2}$ to follow. Define the error variables:

$$
z_{i}=x_{i}-\alpha_{i-1}, \quad i=1,2,3
$$

The problem then is to find $\alpha_{1}, \alpha_{2}$ and $u$ to drive the error variables $z_{i}$ to zero. The control signal is obtained following these steps:

\section{Step 1: Find $\alpha_{1}$}

The dynamics of $z_{1}$, using (4.38) and (4.35)

$$
\dot{z}_{1}=\dot{x}_{1}-\dot{\alpha}_{0}=\dot{x}_{1}=b_{1} x_{2}=b_{1}\left(\alpha_{1}+z_{2}\right)
$$

Let the Lyapounov function for this subsystem be

$$
V_{1}=\frac{1}{2} z_{1}^{2}
$$

its derivative along the trajectory, using (4.38), is:

$$
\dot{V}_{1}=\dot{z}_{1} z_{1}=b_{1} \alpha_{1} z_{1}+b_{1} z_{2} z_{1}
$$


Choose

$$
\alpha_{1}=\alpha_{11} z_{1}=-\frac{k_{1} z_{1}}{b_{1}}
$$

where provide a negative definite term plus an indefinite term provided for next step

$$
\dot{V}_{1}=-k_{1} z_{1}^{2}+b_{1} z_{2} z_{1}
$$

Parameter and trajectory $k_{1}$ and $z_{2}$ will be chosen later to render $\dot{V}_{1}<0$ and hence guaranteeing asymptotic stability of subsystem (4.39).

\section{Step 2: Find $\alpha_{2}$}

Consider now the dynamics of $z_{2}$ and $\alpha_{1}$, using (4.38), (4.36), (4.42), and (4.39). Therefore

$$
\dot{z}_{2}=\dot{x}_{2}-\dot{\alpha}_{1}=b_{2} x_{3}+b_{3} x_{2}+k_{1} \frac{\dot{z}_{1}}{b_{1}}=b_{2} x_{3}+\left(-\frac{k_{1}^{2}}{b_{1}}-\frac{k_{1} b_{3}}{b_{1}}\right) z_{1}+\left(b_{3}+k_{1}\right) z_{2}
$$

The following augmented Lyapounov function is chosen for the system described by (4.39) and (4.44)

$$
V_{2}=V_{1}+\frac{1}{2}\left(z_{2}\right)^{2}
$$

its derivative along the trajectory, using (4.43) and (4.44), is:

$$
\dot{V}_{2}=\dot{V}_{1}+\dot{z}_{2} z_{2}
$$

using (4.43) and (4.44) and let $x_{3}=z_{3}+\alpha_{2}$

$$
\dot{V}_{2}=-k_{1} z_{1}^{2}+\left[b_{1} z_{1}+b_{2} \alpha_{2}-\left(\frac{k_{1}^{2}}{b_{1}}+\frac{k_{1} b_{3}}{b_{1}}\right) z_{1}+\left(b_{3}+k_{1}\right) z_{2}\right] z_{2}+b_{2} z_{2} z_{3}
$$

Choose trajectory $\alpha_{2}$

$$
\alpha_{2}=\alpha_{21} z_{1}+\alpha_{22} z_{2}
$$

where $\alpha_{21}$ and $\alpha_{22}$ are defined by

$$
\begin{aligned}
\alpha_{21} & =\frac{-b_{1}}{b_{2}}+\frac{k_{1}^{2}}{b_{2} b_{1}}+\frac{k_{1} b_{3}}{b_{1} b_{2}} \\
\alpha_{22} & =-\frac{k_{1}+k_{2}+b_{3}}{b_{2}}
\end{aligned}
$$


then, $\dot{V}_{2}$ becomes

$$
\dot{V}_{2}=-k_{1} z_{1}^{2}-k_{2} z_{2}^{2}+b_{2} z_{2} z_{3}
$$

Decision on choosing $\alpha_{2}$ is based on making the term inside the bracket (4.47) negative definite. In following, $k_{1}, k_{2}$, and $z_{3}$ will be chosen to make $\dot{V}_{2}<0$ and hence subsystem (4.39), (4.44) asymptotically stable.

\section{Step 3: Find $u$}

Consider the dynamics of $z_{3}$ and $\alpha_{2}$ using equations (4.38), (4.37), (4.48), (4.39), and (4.44)

$$
\dot{z}_{3}=\dot{x}_{3}-\dot{\alpha}_{2}=\beta u+b_{4} x_{3}+d^{\prime}-\dot{\alpha}_{2}
$$

where $d^{\prime}$ is given in (4.34) and $\dot{\alpha}_{2}$ is obtained using (4.39) and (4.44)

$$
\dot{\alpha}_{2}=\alpha_{21} \dot{z}_{1}+\alpha_{22} \dot{z}_{2}=\left(-k_{1} \alpha_{21}-b_{1} \alpha_{22}\right) z_{1}+\left(b_{1} \alpha_{21}-k_{2} \alpha_{22}\right) z_{2}+\left(b_{2} \alpha_{22}\right) z_{3}
$$

Equation (4.51) becomes

$$
\dot{z}_{3}=\beta u+\left(b_{4} \alpha_{21}+k_{1} \alpha_{21}+b_{1} \alpha_{22}\right) z_{1}+\left(b_{4} \alpha_{22}-b_{1} \alpha_{21}+k_{2} \alpha_{22}\right) z_{2}+\left(b_{4}-b_{2} \alpha_{22}\right) z_{3}+d^{\prime}
$$

Consider the following augmented Lyapounov function for (4.39), (4.44), and (4.53)

$$
V=V_{2}+\frac{1}{2}\left(z_{3}\right)^{2}+\frac{1}{2}(\beta-\hat{\beta})^{2} \gamma^{-1}+\frac{1}{2}\left[\begin{array}{lll}
\left(\theta_{1}-\hat{\theta}_{1}\right) & \left(\theta_{2}-\hat{\theta}_{2}\right)
\end{array}\right] \Gamma^{-1}\left[\begin{array}{ll}
\left(\theta_{1}-\hat{\theta}_{1}\right) & \left(\theta_{2}-\hat{\theta}_{2}\right)
\end{array}\right]^{T}
$$

where $\hat{\theta}_{1}, \hat{\theta}_{2}$, and $\hat{\beta}$ are estimate of $\theta_{1}, \theta_{2}, \beta . \Gamma=\operatorname{diag}\left[\Gamma_{1}, \Gamma_{2}\right]$ is an adaptation gain matrix, and $\gamma$ is a scalar positive value. Then

$$
\begin{aligned}
\dot{V} & =-k_{1} z_{1}^{2}-k_{2} z_{2}^{2}+\left[\beta u+\alpha_{31} z_{1}+\alpha_{32} z_{2}+\alpha_{33} z_{3}+d^{\prime}\right] z_{3}- \\
& -(\beta-\hat{\beta}) \gamma^{-1} \dot{\hat{\beta}}-\left(\theta_{1}-\hat{\theta}_{1}\right) \Gamma_{1}^{-1} \dot{\hat{\theta}}_{1}-\left(\theta_{2}-\hat{\theta}_{2}\right) \Gamma_{2}^{-1} \dot{\hat{\theta}}_{2}
\end{aligned}
$$

where

$$
\begin{aligned}
\alpha_{31} & =b_{4} \alpha_{21}+k_{1} \alpha_{21}+b_{1} \alpha_{22} \\
\alpha_{32} & =b_{2}-b_{1} \alpha_{1}+b_{4} \alpha_{22}+k_{2} \alpha_{22} \\
\alpha_{33} & =b_{4}-b_{2} \alpha_{22}
\end{aligned}
$$


Now consider $d^{\prime}$ as given in equation (4.34). The adaptation laws $\dot{\hat{\beta}}, \dot{\hat{\theta}}_{1}, \dot{\hat{\theta}}_{2}$ in (4.55) are chosen in a way to obtain (4.55) independent of $\theta_{1}, \theta_{2}, \hat{\beta}$ :

$$
\begin{aligned}
\dot{\hat{\theta}}_{1} & =\Gamma_{1} z_{3} x_{3} \\
\dot{\hat{\theta}}_{2} & =\Gamma_{2} z_{3} \dot{x}_{3} \\
\dot{\hat{\beta}} & =\gamma z_{3} u
\end{aligned}
$$

Adaptation laws 4.57 are obtained as follow. Consider equation 4.55

$$
\begin{aligned}
\dot{V} & =-k_{1} z_{1}^{2}-k_{2} z_{2}^{2}+\beta u z_{3}+\alpha_{31} z_{1} z_{3}+\alpha_{32} z_{2} z_{3}+\alpha_{33} z_{3}^{2}+d^{\prime} z_{3}- \\
& -\beta \gamma^{-1} \dot{\hat{\beta}}+\hat{\beta} \gamma^{-1} \dot{\hat{\beta}}-\theta_{1} \Gamma_{1}^{-1} \dot{\hat{\theta}}_{1}+\hat{\theta}_{1} \Gamma_{1}^{-1} \dot{\hat{\theta}}_{1}-\theta_{2} \Gamma_{2}^{-1} \dot{\hat{\theta}}_{2}+\hat{\theta}_{2} \Gamma_{2}^{-1} \dot{\hat{\theta}}_{2}
\end{aligned}
$$

substitute $d^{\prime}=\theta_{1} x_{3}+\theta_{2} \dot{x}_{3}$ and factorize $\theta_{1}, \theta_{2}$ and $\beta$. Equation (4.58) becomes

$$
\begin{aligned}
\dot{V} & =-k_{1} z_{1}^{2}-k_{2} z_{2}^{2}+\beta\left(u z_{3}-\gamma^{-1} \dot{\hat{\beta}}\right)+\alpha_{31} z_{1} z_{3}+\alpha_{32} z_{2} z_{3}+\alpha_{33} z_{3}^{2}+ \\
& +\theta_{1}\left(x_{3} z_{3}-\Gamma_{1}^{-1} \dot{\hat{\theta}}_{1}\right)+\theta_{2}\left(\dot{x}_{3} z_{3}-\Gamma_{2}^{-1} \dot{\hat{\theta}}_{2}\right)+\hat{\beta} \gamma^{-1} \dot{\hat{\beta}}+\hat{\theta}_{1} \Gamma_{1}^{-1} \dot{\hat{\theta}}_{1}+\hat{\theta}_{2} \Gamma_{2}^{-1} \dot{\hat{\theta}}_{2}
\end{aligned}
$$

Since $\theta_{1}, \theta_{2}$, and $\beta$ are unknown, terms related to them are canceled. Set

$$
\begin{aligned}
x_{3} z_{3}-\Gamma_{1}^{1} \dot{\hat{\theta}}_{1} & =0 \\
u z_{3}-\gamma^{-1} \dot{\hat{\beta}} & =0 \\
\dot{x}_{3} z_{3}-\Gamma_{2}^{-1} \dot{\hat{\theta}}_{2} & =0
\end{aligned}
$$

Equation (4.59) becomes

$$
\dot{V}=-k_{1} z_{1}^{2}-k_{2} z_{2}^{2}+\alpha_{31} z_{1} z_{3}+\alpha_{32} z_{2} z_{3}+\alpha_{33} z_{3}^{2}+\hat{\beta} \gamma^{-1} \dot{\hat{\beta}}+\hat{\theta}_{1} \Gamma_{1}^{-1} \dot{\hat{\theta}}_{1}+\hat{\theta} \Gamma_{2}^{-1} \dot{\hat{\theta}}_{2}
$$

Substitute (4.57) in (4.62)

$$
\dot{V}=-k_{1} z_{1}^{2}-k_{2} z_{2}^{2}+\alpha_{31} z_{1} z_{3}+\alpha_{32} z_{2} z_{3}+\alpha_{33} z_{3}^{2}+\hat{\beta} u z_{3}+\hat{\theta}_{1} x_{3} z_{3}+\hat{\theta} \dot{x}_{3} z_{3}
$$

factorizing $z_{3}$

$$
\dot{V}=-k_{1} z_{1}^{2}-k_{2} z_{2}^{2}+\left[\hat{\beta} u+\alpha_{31} z_{1}+\alpha_{32} z_{2}+\alpha_{33} z_{3}+\hat{d}\right] z_{3}
$$

where $\hat{d}=\hat{\theta}_{1} x_{3}+\hat{\theta}_{2} \dot{x}_{3}$. The controller $u$ is then designed to make $\dot{V}<0$. This is achieved with the following controller and positive values $k_{1}, k_{2}, k_{3}$ :

$$
u=\hat{\beta}^{-1}\left[-\alpha_{31} z_{1}-\alpha_{32} z_{2}-\alpha_{33} z_{3}-k_{3} z_{3}-\hat{d}\right]
$$


$\hat{\beta} \neq 0$ for normal operating conditions, and make the controller feasible. In fact, with this controller, $\dot{V}$ is expressed by:

$$
\dot{V}=-k_{1} z_{1}^{2}-k_{2} z_{2}^{2}-k_{3} z_{3}^{2}
$$

Finally, this control law is written in terms of the original state variables as

$$
u=\hat{\beta}^{-1}\left[F_{1} x_{1}+F_{2} x_{2}+F_{3} x_{3}-\hat{d}\right]
$$

where

$$
\begin{gathered}
F_{1}\left(k_{1}, k_{2}, k_{3}\right)=\left[-\alpha_{31}+\alpha_{21} \alpha_{33}+k_{3} \alpha_{21}+\frac{-k_{1} \alpha_{32}+k_{1} \alpha_{22} \alpha_{33}+k_{1} k_{3} \alpha_{22}}{b_{1}}\right] \\
F_{2}\left(k_{1}, k_{2}, k_{3}\right)=\left[-\alpha_{32}+\alpha_{33} \alpha_{22}+k_{3} \alpha_{22}\right] \\
F_{3}\left(k_{1}, k_{2}, k_{3}\right)=\left[-\alpha_{33}-k_{3}\right]
\end{gathered}
$$

This controller $u\left(x_{1}, x_{2}, x_{3}, \hat{\theta}_{1}, \hat{\theta}_{2}, \hat{\beta}\right)(4.66)$ is a nonlinear function that is affected by the choice of the control parameters:

$$
K=\left[\begin{array}{llllll}
k_{1} & k_{2} & k_{3} & k_{4} & k_{5} & k_{6}
\end{array}\right]
$$

where: $k_{4}=\Gamma_{1}, k_{5}=\Gamma_{2}, k_{6}=\gamma$.

\subsubsection{Optimal Settings for Controller Gains}

Following a system disturbance, the main objective of damping controllers is to prevent loss of synchronism and withstand large deviations of states from their nominal. In order to achieve this goal, effective controllers need to act fast. Improperly design or tuned controllers may contribute to unstability problem. Hence in selecting controller gains, emphasis is placed on stability of all the system.

The controller designed Subsection 4.3.3 is at each machine and the control gains (4.70) are denoted $K_{i}=\left[\begin{array}{llllll}k_{i 1} & k_{i 2} & k_{i 3} & k_{i 4} & k_{i 5} & k_{i 6}\end{array}\right]$. If the system comprises $n$ substations, then there are $N=6 n$ control parameters that need to be selected simultaneously so that each substation is asymptotically stable. A vector Lyapounov Function for the entire system is

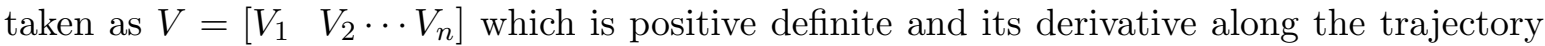




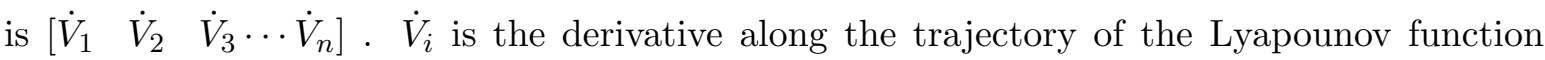
of subsystem $(i)$ given by (4.65). Therefore, the goal is to minimize the objective function $J=\left[\begin{array}{llll}J_{1} & J_{2} & \cdots & J_{n}\end{array}\right]$, where $J_{i}=\dot{V}_{i}$ for $i=1,2, \cdots n$, i.e. to make each entry of $J$ as negative as possible by tuning the control gains. The problem is formulated as follows:

$$
\max _{K_{i} \text { such that } \dot{V}_{i}<0, i=1: n}\|J\|_{\infty}
$$

Given the size of the problem, and the system complexities, this problem is solved using the Particle Swarm Optimization (PSO). Advantages with this approach are

- Appropriate Lyapunov function (4.54) is obtained through adaptive backstepping procedure

- With optimization technique, adaptive backstepping controller maximizes (4.65)

- Adaptation laws (4.57) are obtained to estimate interface variables locally

- Local control signals are used to implement control structure (4.66)

\subsubsection{Case Study}

\section{Fifty Machine System}

Fifty machine system, as explained in 4.24, is considered as case study [31]. Adaptive backstepping controllers (4.66), are implemented as supplementary damping signal to excitation system for generators 2 and 6 at related buses 104 and 111 respectively.

\section{Excitation Control Design}

Following figure shows schematic block diagram of adaptive backstepping control for a generator with Exciter. Exciter consists of amplifier gain $K_{A}$, amplifier time constant $T_{A}$, saturation hard limit, actuator gain $K_{E}$, actuator time constant $T_{E}$. Parameters for exciter is given in appendix D.

\section{Scenario-I}

The ability of the system to tolerate a three phase fault on transmission line $6-9$ is investigated. For this scenario the system is analyzed in two operating conditions. First the configuration of system as shown in Figure 4.7. And second, the system with the line $6-9$ 


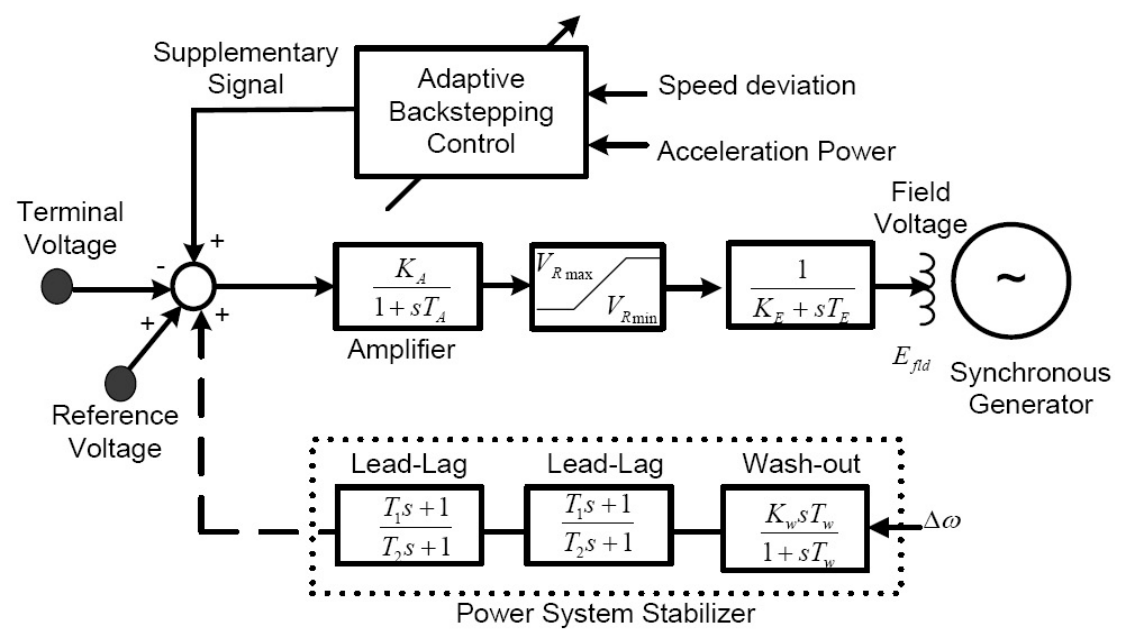

Figure 4.14: Adaptive backstepping Controller with Exciter

out of service for $250 \mathrm{msec}$. The control and adaptation gains are obtained in a way that satisfy (4.71) for generators 2,6. Figure (4.15) compares performance of exciter with proposed controller, exciter with power system stabilizer, and exciter without supplementary signal. Proposed controller damp the oscillation of rotor angles more effectively in comparison to PSS. The PSS for each generator (2 and 6) has a wash-out stage and two identical lead-lag stages. Control and adaptation gains are given in Tables 4.11 and 4.12.

Table 4.8: Adaptive Backstepping Controller Gains

\begin{tabular}{c|c|c|c}
\hline Backstepping & $k_{1}$ & $k_{2}$ & $k_{3}$ \\
\hline Gen \#2 & 67.45 & 36.87 & 116.05 \\
Gen \#6 & 151.04 & 7.91 & 0.93 \\
\hline Adaptation gains & $k_{4}$ & $k_{5}$ & $k_{6}$ \\
\hline Gen \#2 & 359.90 & 402.00 & 275.07 \\
Gen \#6 & 557.40 & 30.80 & 53.15 \\
\hline
\end{tabular}

Table 4.9: PSS Gains

\begin{tabular}{c|c|c|c|c}
\hline PSS & $T_{w}$ & $K_{w}$ & $T_{1}$ & $T_{2}$ \\
\hline Gen \#2 & 3.18 & 14.85 & 0.71 & 0.49 \\
Gen \#6 & 6.92 & 7.75 & 1.33 & 1.10 \\
\hline
\end{tabular}



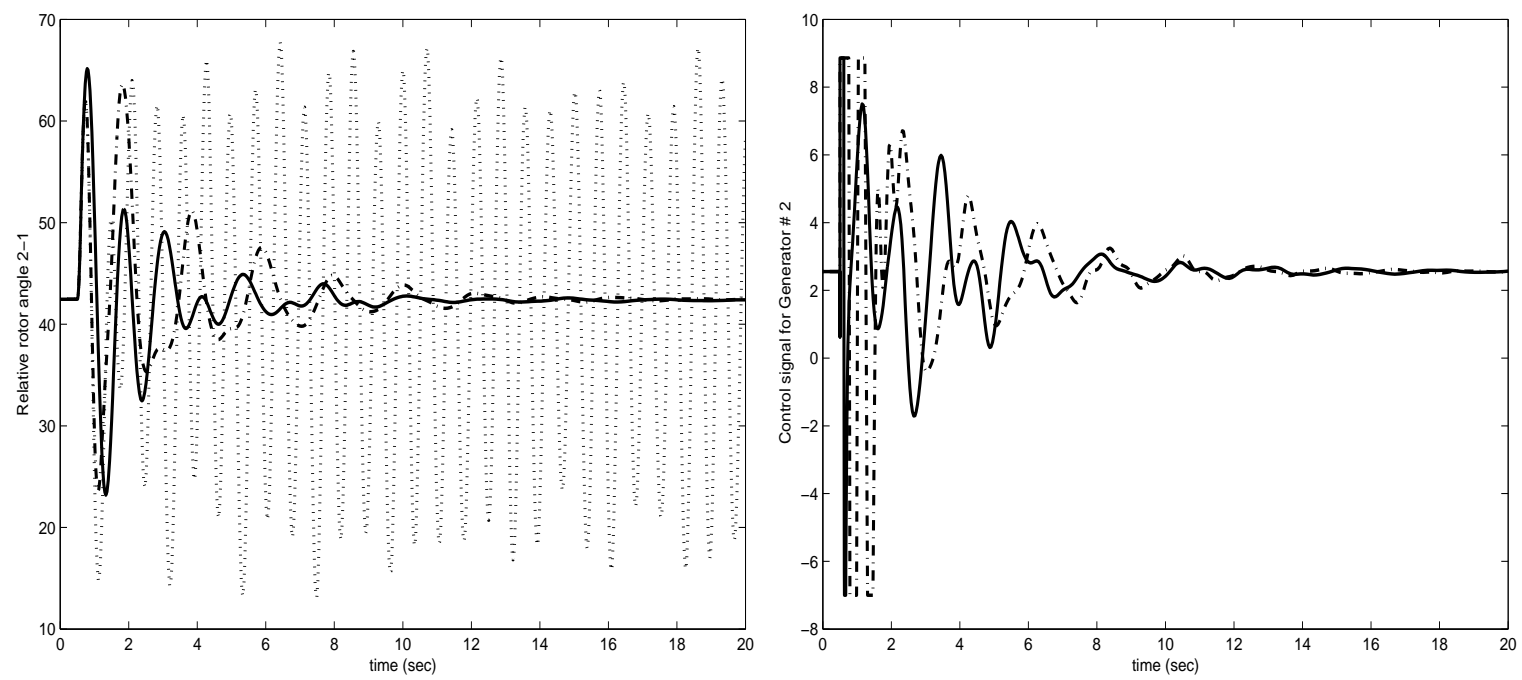

Figure 4.15: Relative rotor angle $\delta_{21}$ and control signal Generator 2. Adaptive Backstepping (solid line -), and Power System Stabilizer (dash-dot line -.), Exciter without supplementary signal (dot line.)
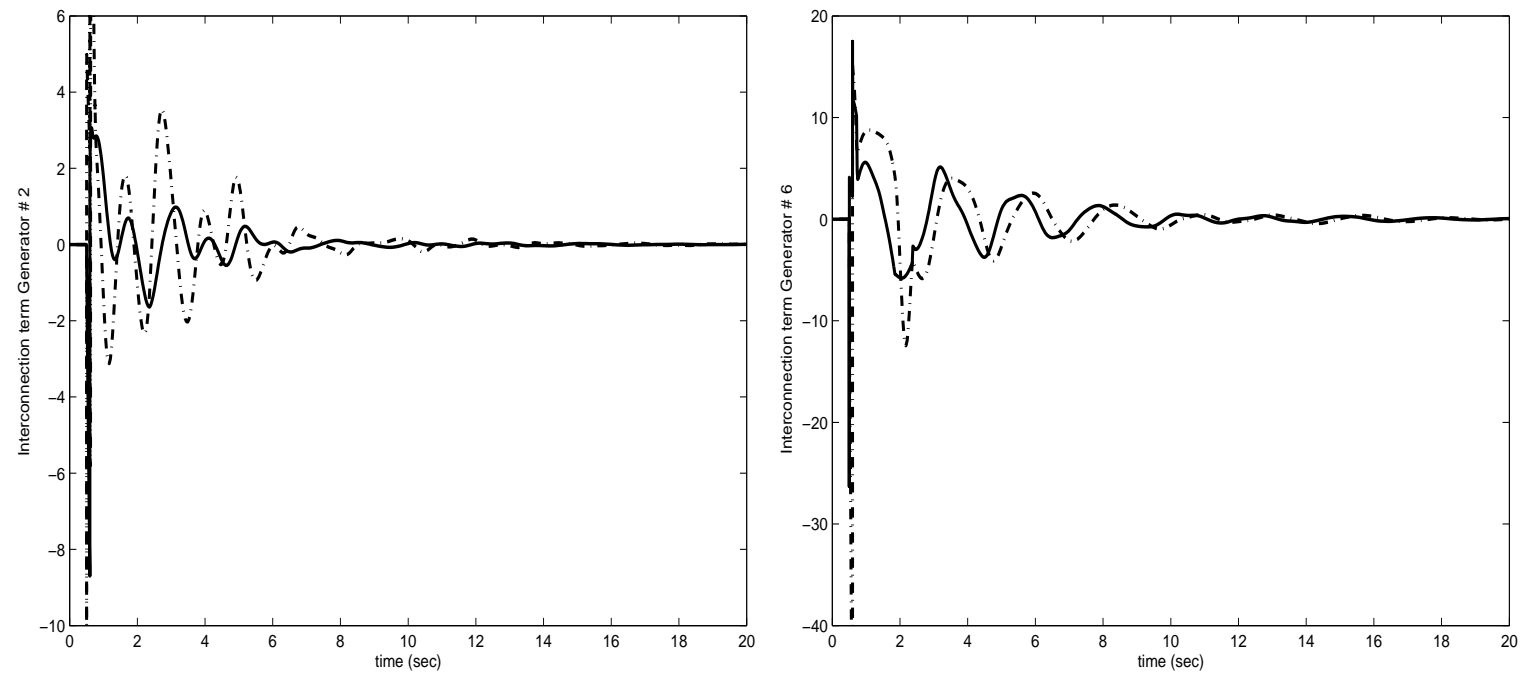

Figure 4.16: Interface modeling for generators 2 and 6. Exact term d' (solid line -), estimated term $\hat{d}$ (dash dot line -.) 

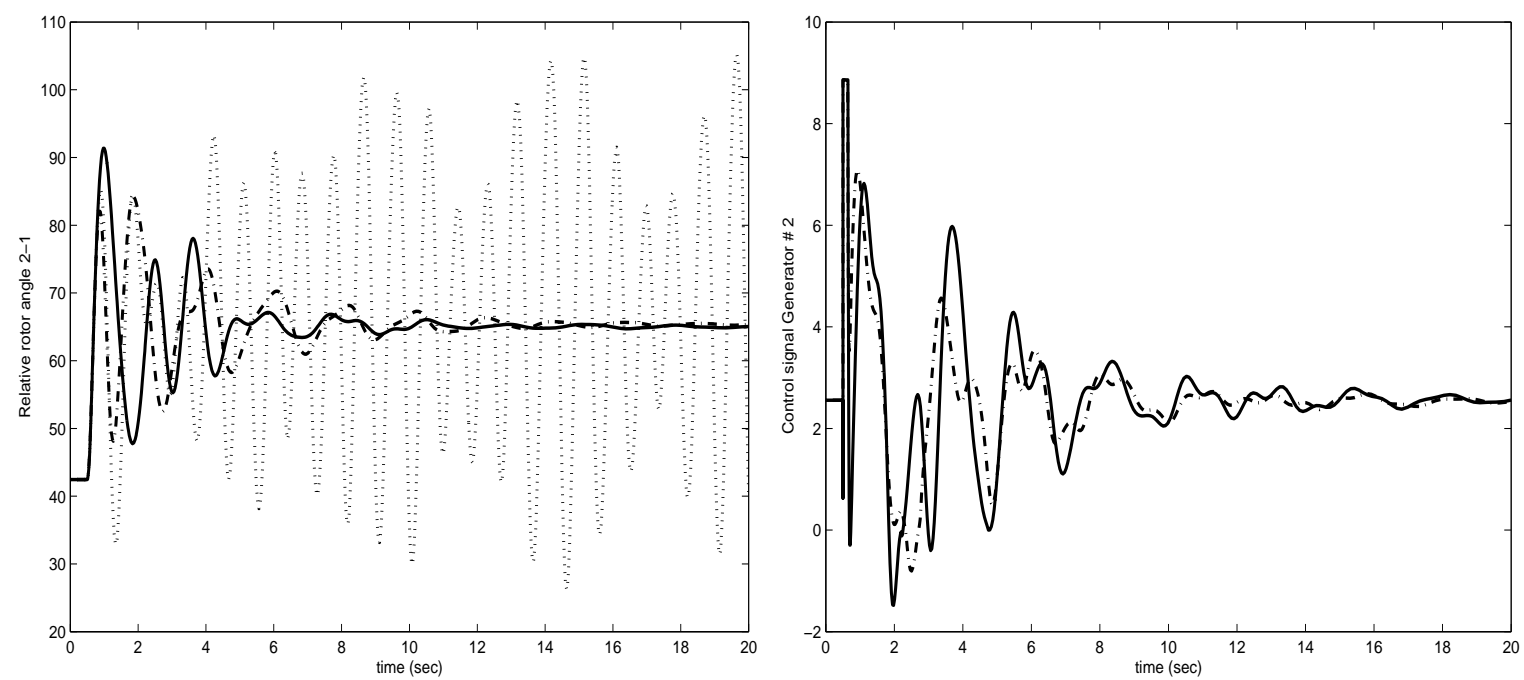

Figure 4.17: Relative rotor angle $\delta_{21}$ (deg) and Control signal generator 2. Adaptive Backstepping (solid line -), PSS (dot line :)

\section{Scenario-II}

Consider the same gains from Table 4.11, but type and location of fault is altered (Line to ground Fault on line $6-7$ at 0.5 second, the transmission line remains open). Adaptive backstepping control is applied for generators 2 and 6 at buses 104 and 111. Figures (4.18) compares the coupling terms, exact term in dot-line (4.25) and estimated interface variables with solid line (4.34) for generator 2 and 6 . The adaptation laws adjusts fraction of incoming power and it is being used by backstepping control to produce effective control signals which damp oscillations and stabilize the system.

In this section PSO based optimization algorithm for damping control design with relatively large scale system is presented. The PSO algorithm allows simultaneous tuning of multiple power system nonlinear controllers in different operating conditions. Two different scenarios were presented for this system, three phase fault and loss of transmission line. The obtained results show that PSO can be effectively utilized for nonlinear damping controllers with their local settings at two generators 2 and 6 . Furthermore, comparison with conventional PSS shows that the performance of the proposed technique is superior. 

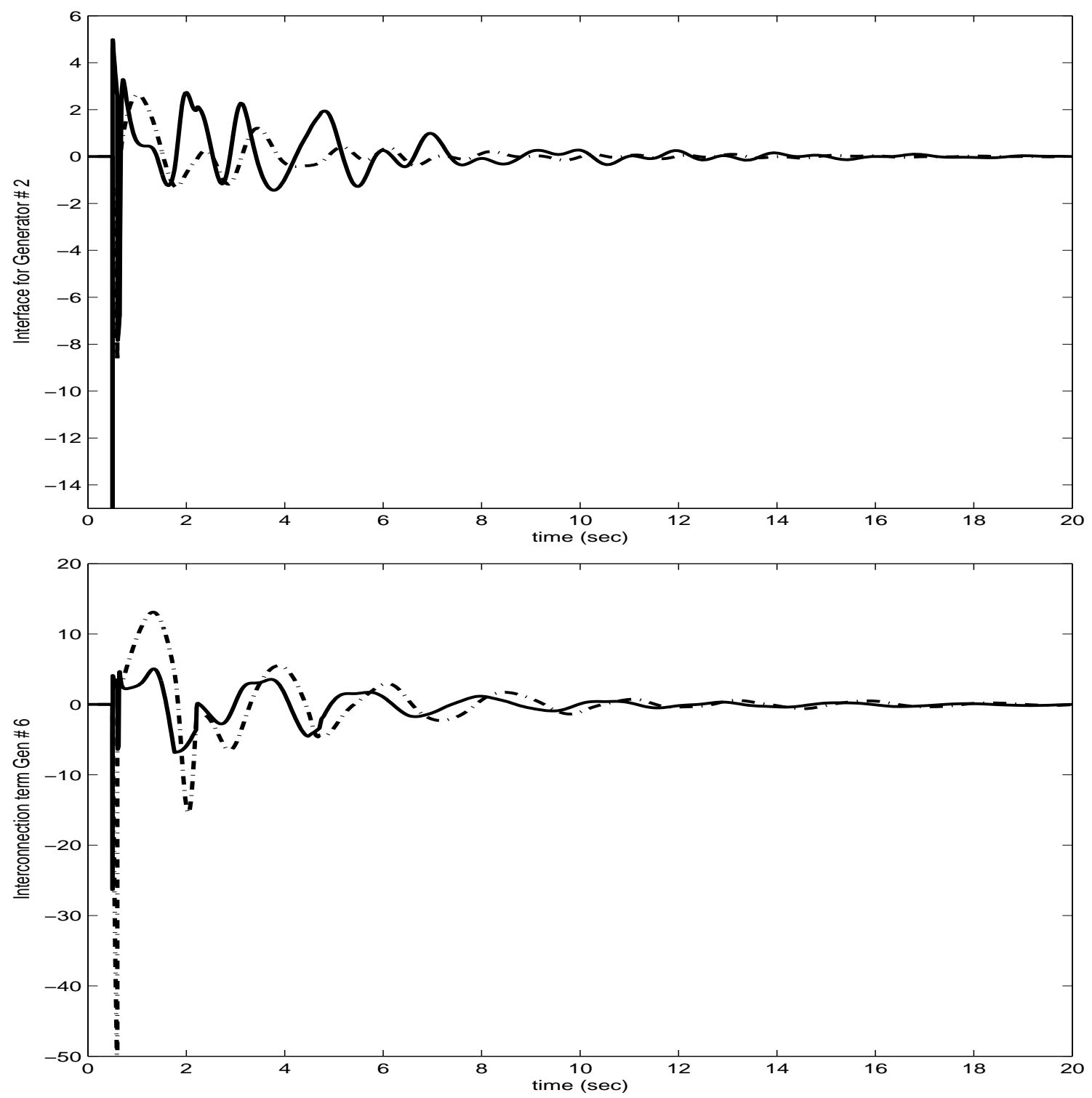

Figure 4.18: Interface modeling for generators 2 and 6. Exact term d' (solid line-), estimated term $\hat{d}$ (dash dot line -.) 


\section{Two Area System:}

Second system is the benchmark two-area power system (Figure 4.19) that has been extensively used in the literature for analyzing inter-area oscillations [61].

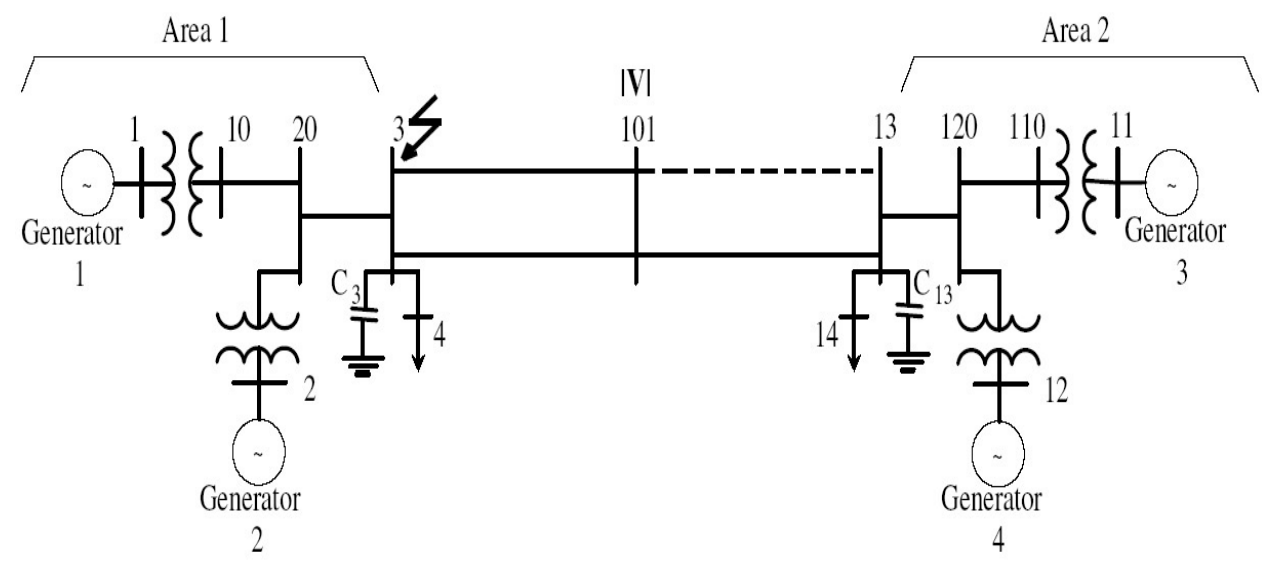

Figure 4.19: Two area system

\section{Excitation Control Design}

Figure 4.20 shows the schematic block diagram of the proposed adaptive backstepping controller acting as supplementary signal. The actuator consists of an exciter gain $K_{A}$, an time constant $T_{A}$, a filter time constant $T_{r}$ and a saturation hard limit with upper and lower bounds $\left[\begin{array}{ll}V_{R_{\min }} & V_{R_{\max }}\end{array}\right]$. Excitation data is given in Table 6.5.

Table 4.10: Parameters for Static Excitation

\begin{tabular}{c|c}
\hline Exciter & $1,2,3,4$ \\
\hline$K_{A}$ Regulator gain p.u. & 200 \\
\hline$T_{A}$ Regulator time constant p.u. & 0.05 \\
\hline$T_{r}$ Filter time constant p.u. & 0.01 \\
\hline$V_{R \max }$ upper bound for saturation p.u. & 10 \\
\hline$V_{\text {Rmin }}$ lower bound for saturation p.u. & -10 \\
\hline
\end{tabular}




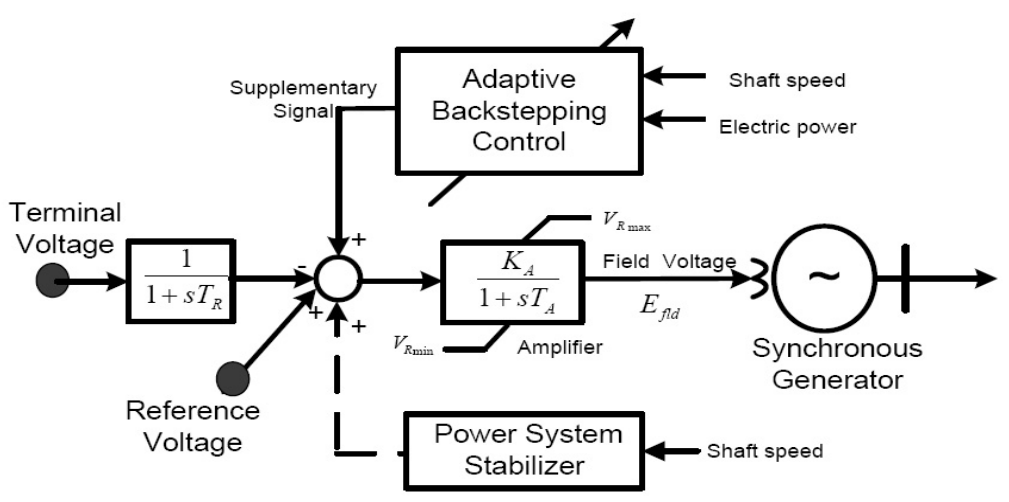

Figure 4.20: Adaptive backstepping Controller with static exciter

Table 4.11: PSS Gains

\begin{tabular}{c|c|c|c|c}
\hline PSS & $T_{w}$ & $K_{w}$ & $T_{1}$ & $T_{2}$ \\
\hline Gen \#2 & 20 & 2.36 & 0.7109 & 0.155 \\
Gen \#3 & 20 & 15 & 0.15 & 0.0843 \\
\hline
\end{tabular}

\section{Scenarios}

Scenarios are presented to illustrate the effectiveness of the proposed controller and to compare it to existing PSS. PSS at generators 2 and 3 have been successfully designed by authors previously to damp local and inter-area oscillations [37]. The data for generators are given in Appendix C. Following control strategies are analyzed:

- Two PSSs, whose transfer functions are given below, are implemented on generators 2 and 3 .

$$
\underbrace{K_{w} \frac{s T_{w}}{1+s T_{w}}}_{\text {Wash-out }} \underbrace{\frac{\left(1+s T_{1}\right)^{2}}{\left(1+s T_{2}\right)^{2}}}_{\text {Lead-Lag }}
$$

Where $T_{w}$ wash-out time constant, $K_{w}$ wash-out gain, and $T_{1}, T_{2}$ are lead-lag time constants. PSS parameters are given in Table 4.11 [37].

- Two Adaptive Backstepping controllers, designed using the proposed approach, are implemented on generators 2 and 3. Gains, tuned by PSO, are given in Table 4.12. Here, interconnection coupling term $d_{i}^{\prime}$ is approximated locally with a second order polynomial function of 
Table 4.12: Adaptive Backstepping Controller Gains

\begin{tabular}{c|c|c|c}
\hline Backstepping & $k_{1}$ & $k_{2}$ & $k_{3}$ \\
\hline Gen \#2 & 2.40 & 1.27 & 19.34 \\
Gen \#3 & 10.73 & 16.76 & 28.20 \\
\hline Adaptation gains & $k_{4}$ & $k_{5}$ & $k_{6}$ \\
\hline Gen \#2 & 13.5 & 14.41 & 10.72 \\
Gen \#3 & 1.15 & 3.81 & 11.34 \\
\hline
\end{tabular}

Table 4.13: Analyzed Scenarios

\begin{tabular}{c|c|c|c}
\hline Scenarios \# & I & II & III \\
\hline Transmission line $(13-101)$ & double & single & single \\
\hline Fault occurrence time $(\mathrm{sec})$ & 1.00 & 1.00 & 1.00 \\
\hline Fault clearance time $(\mathrm{sec})$ & 1.026 & 1.046 & 1.06 \\
\hline Line re-closing time $(\mathrm{sec})$ & 1.03 & 1.05 & 10.00 \\
\hline
\end{tabular}

electric power deviation. Its parameters are estimated, using local information:

$$
d_{i}^{\prime} \approx \theta_{1 i} \Delta P_{e_{i}}+\theta_{2 i} \Delta P_{e_{i}}^{2}
$$

where $\theta_{1 i}$ and $\theta_{i 2}$ are values estimated using adaptation laws. The modification is done in (4.55) where adaptation laws are denoted as:

$$
\begin{aligned}
\dot{\hat{\theta}}_{1} & =\Gamma_{1} z_{3} x_{3} \\
\dot{\hat{\theta}}_{2} & =\Gamma_{2} z_{3} x_{3}^{2} \\
\dot{\hat{\beta}} & =\gamma z_{3} u
\end{aligned}
$$

The scenarios that have been analyzed are tabulated in Table 4.13. A three phase fault is applied at bus 3 for each scenario. In scenario-I, simulation results show that relative rotor angle oscillations $\Delta \delta_{31}, \Delta \delta_{41}$ is damped fast enough, in less than 8 seconds (Figure 4.21).

In scenario-II, when one transmission line between buses $13-101$ is removed, the system is under stress. Adaptive backstepping controller dampen rotor oscillation in approximately 8 sec, while it takes almost twice that time for PSS to suppress the oscillations (Figure 4.22). 

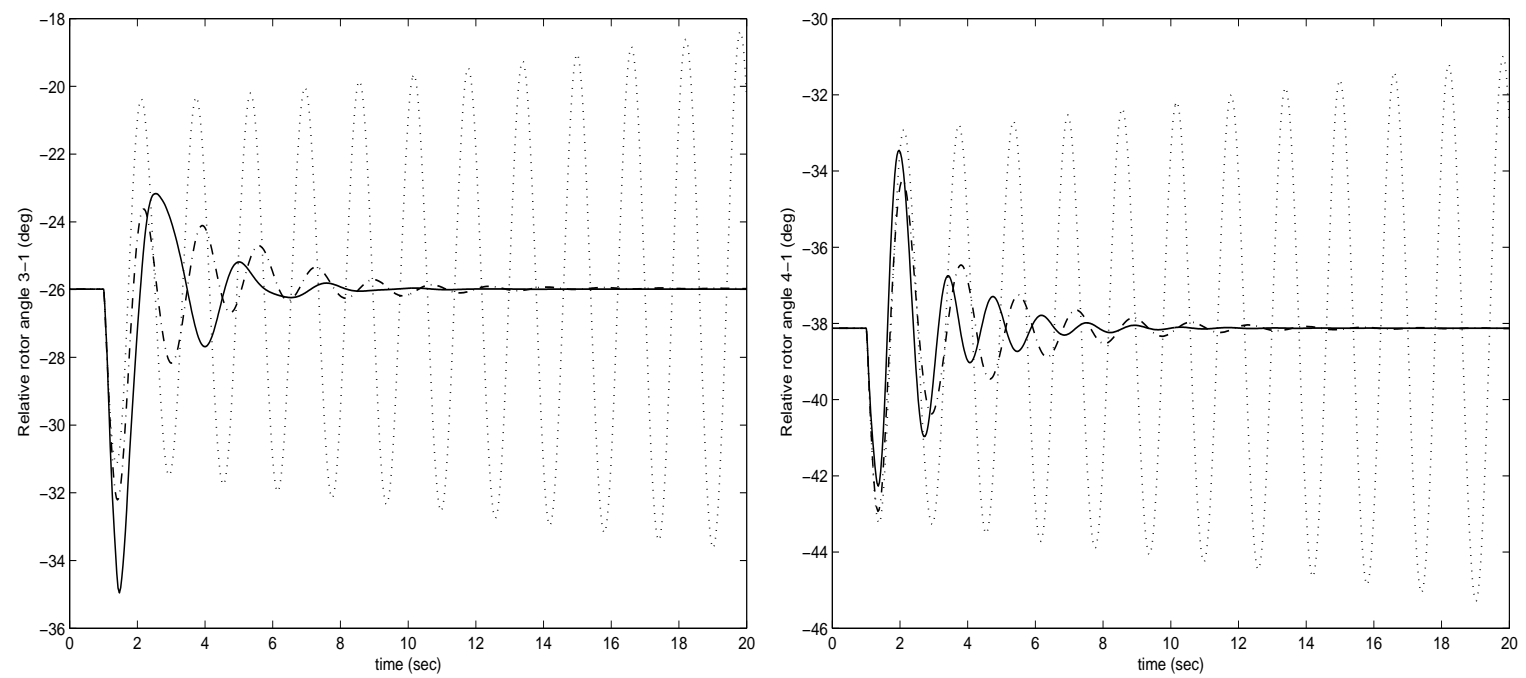

Figure 4.21: Relative rotor angles $\delta_{31}, \delta_{41}$. Adaptive backstepping (solid line), PSS (dash-dotted line) and uncontrolled system (dotted line) for Scenario-I
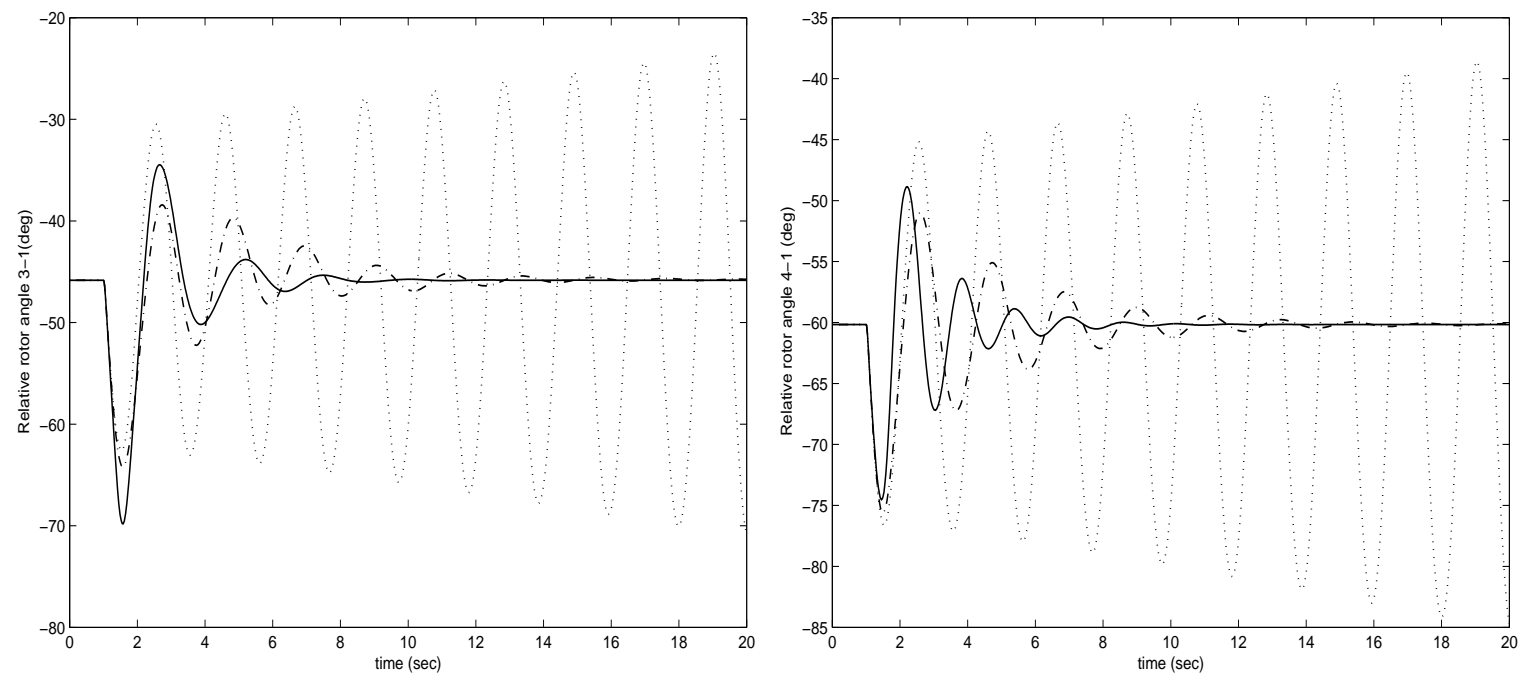

Figure 4.22: Relative rotor angles $\delta_{31}, \delta_{41}$. Adaptive backstepping (solid line), PSS (dash-dotted line) and uncontrolled system (dotted line) for Scenario-II 

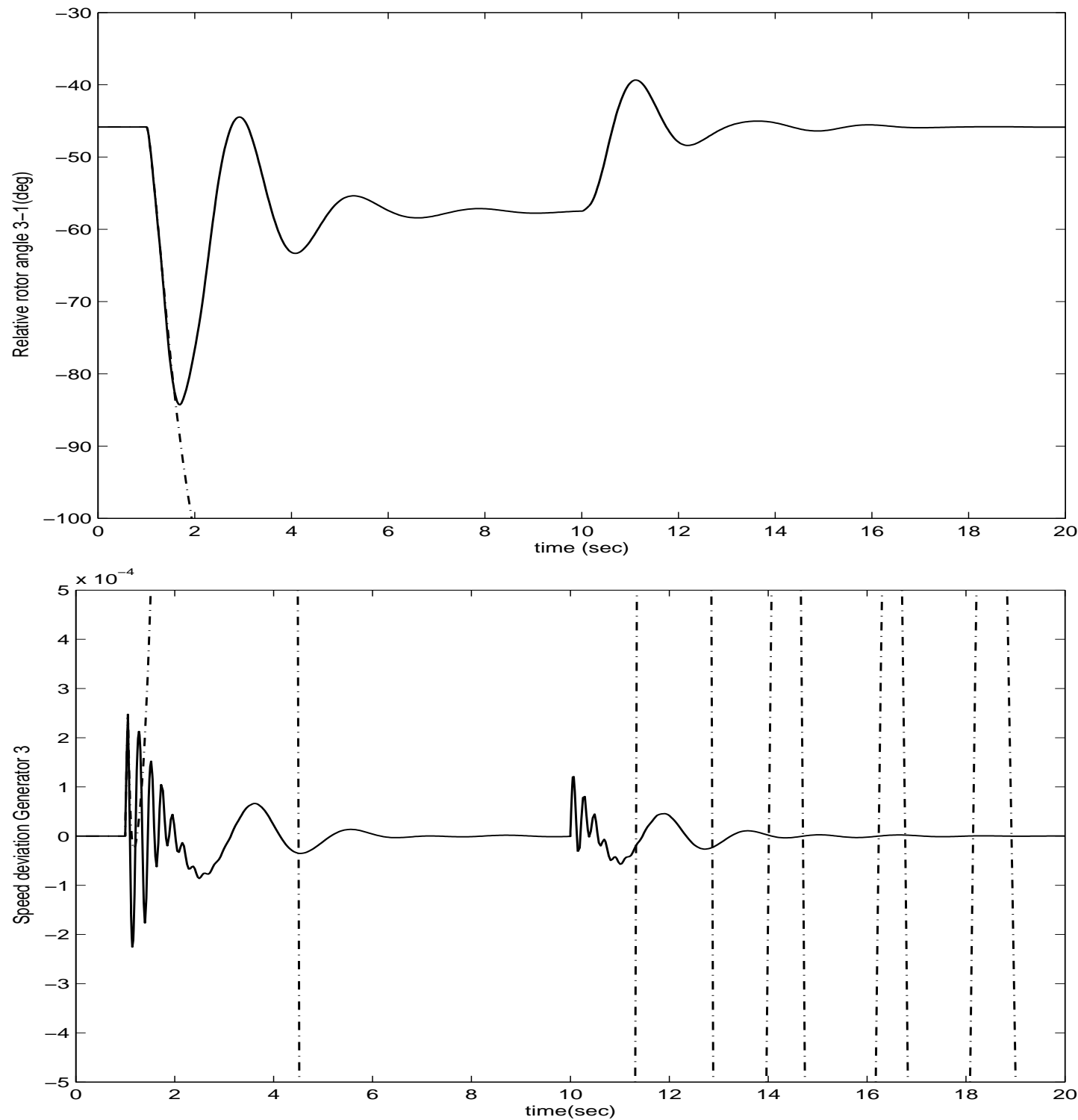

Figure 4.23: Relative rotor angle $\delta_{31}$, Speed deviations $\Delta \omega_{3}$ for Generator 3. Adaptive Backstepping (solid line -), and PSS (dash-dot line-.) in Scenario-III

In scenario-III, unlike aforementioned scenarios, the fault duration is increased. The transmission line is removed at $1.06 \mathrm{sec}$. The transmission line is reconnected at 10 seconds. Proposed controllers stabilize the system which returns to its pre-fault equilibrium point. Relative rotor angle $\delta_{31}, \delta_{41}$ depicts the fact that pre-fault steady state and post-fault steady state are equal (Figure 4.24). Instability, with PSS controller, is the result of low frequency inter-area oscillations that arise due to long duration of fault. 

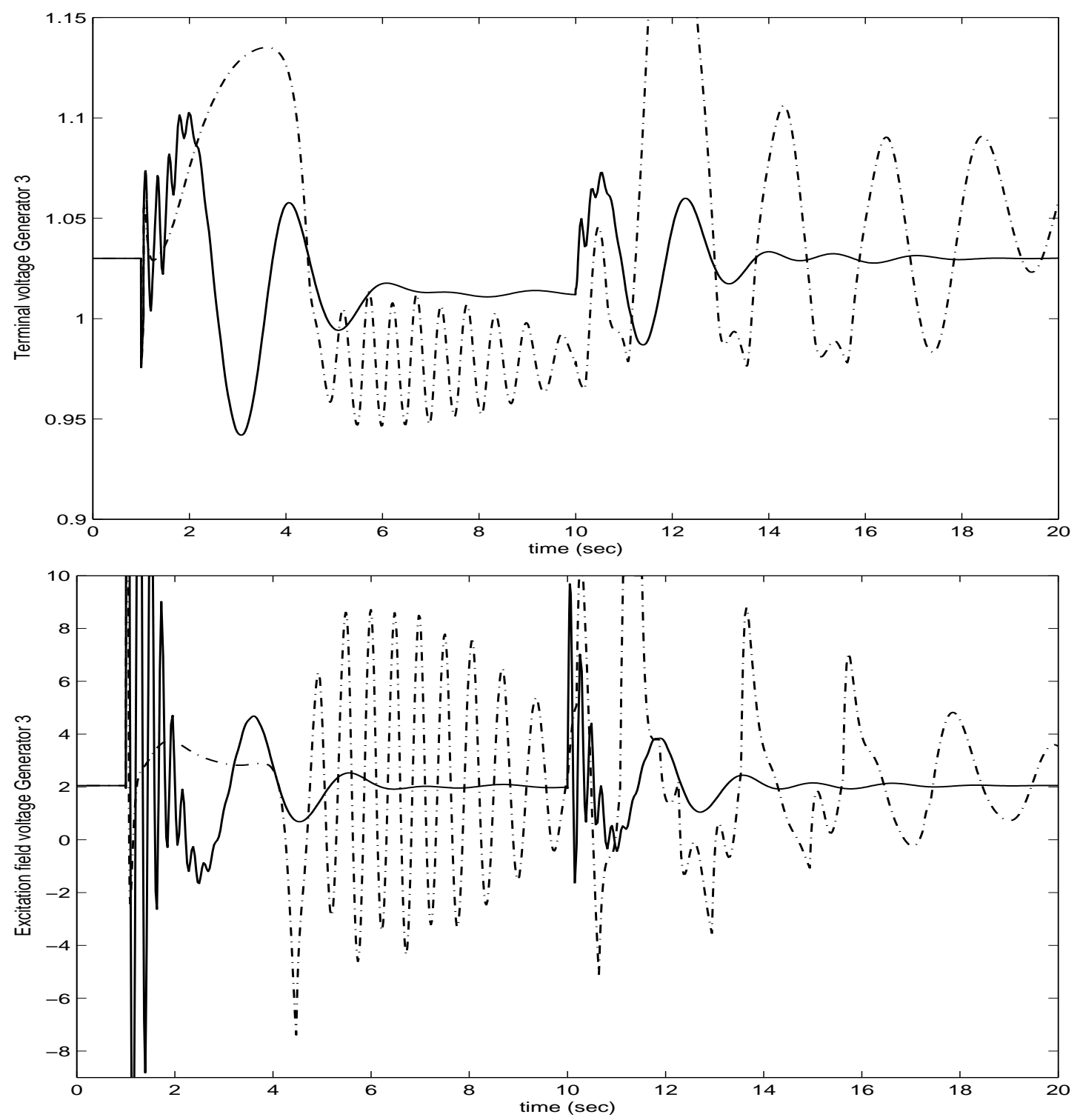

Figure 4.24: Terminal voltage at buses 11 and excitation field voltage $E_{f l d_{3}}$ for Generator 3 . Adaptive Backstepping (solid line -), and PSS (dash-dot line -.) in Scenario-III 
Finally Figures (4.25) compares the estimated and actual $d$ and $\hat{d}$ coupling terms. The adaptation laws adjust the fraction of incoming power to decouple each generator from the effects of the rest of the system, and produce decentralized control signals. Simulation results reveal that the system is well damped and stable during and after large disturbances occur in the system.
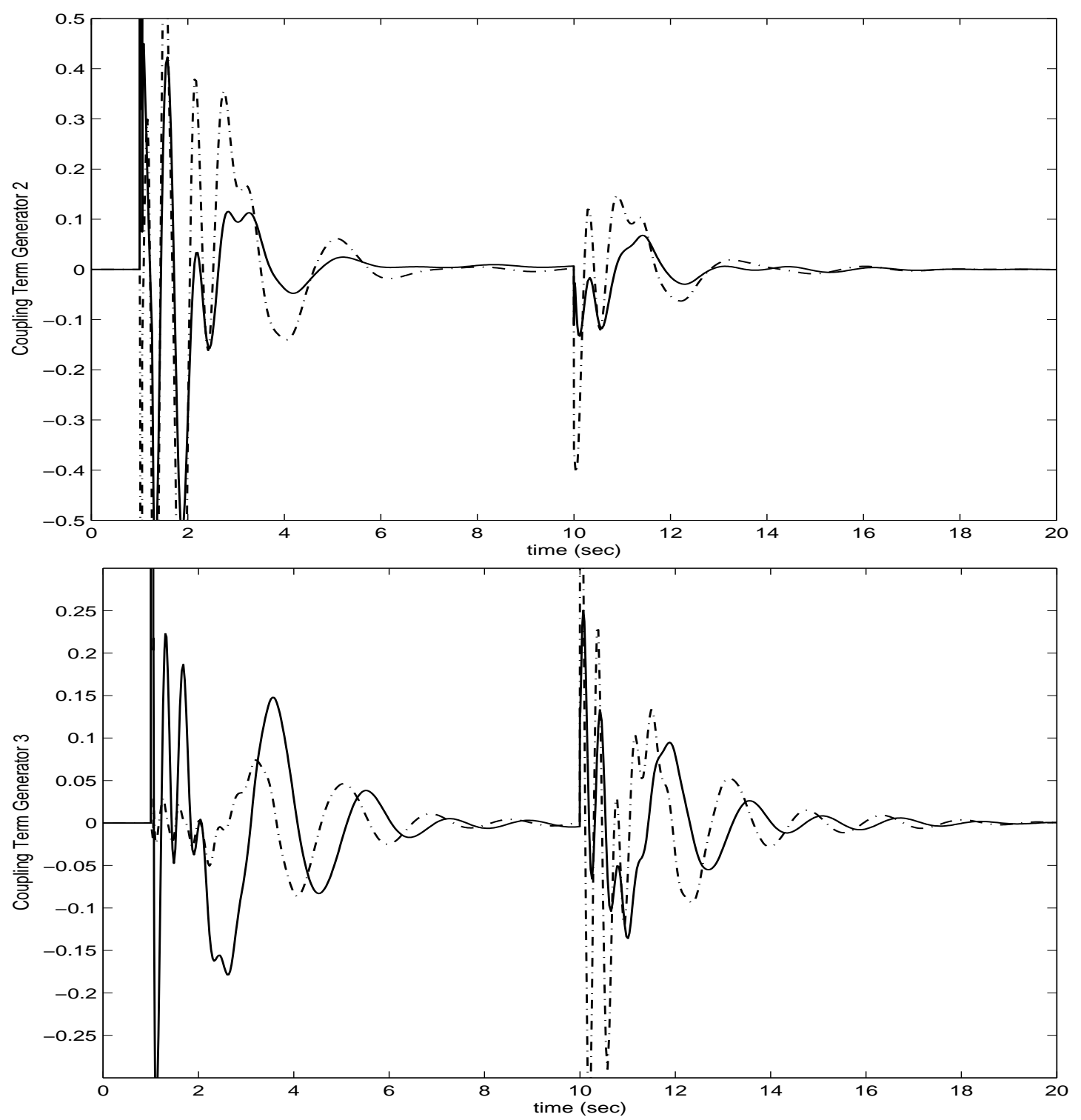

Figure 4.25: Interface modeling for generator 2 and 3. Exact term (solid line -) and estimated term (dash-dot line -.) in Scenario-III

The proposed technique presented an adaptive controller for improving the damping of 
oscillations in a power system during large disturbances. This controller is decentralized, that has been achieved by estimating the external system as uncertain linear function (4.73). The linear estimate of the disturbance has given adequate results, and the global optimization that is performed, counteract any errors in this estimate. Simultaneous optimization is performed to produce the minimum objective function for the over all system under the structure that each local control act as local optimizer. The well damped optimal nonlinear control illustrates the successful solution of the problem, indicating that the technique is a valuable tool dealing with transient control problems for power system.

\subsubsection{Modeling Error}

In reality the dynamic environment for large scale system has uncertainties. Some factors that might contribute to this are listed as follow:

- Operating conditions might be different from those considered in planning and designing stage

- Planning models are inaccurate with respect to system dynamics

- Large changes in system operating conditions might happened due to outside disturbances

Performance of controller is evaluated with respect to generator modeling error. Important requirement is that the tuning procedure must ensure that the proposed damping controller tuned are robust enough to changes in system parameters and they provide adequate damping for a range of parameter changes on the system. To verify the robustness of the optimized tuning controller, scenario-II is investigated as given in Table 4.13. A random change of generator parameters from their nominal values within the range of $\pm 50 \%$ are considered for the

following parameters of each generator $\left(\Delta x_{q}^{\prime}, \Delta T_{d o}^{\prime}, \Delta x_{d}^{\prime}, \Delta T_{q o}^{\prime}, \Delta H\right)$. Figure 4.26 shows speed deviation for generator 3 for several number of simulations. Proposed controller numerically shows robust performance due to modeling errors for all. The mean (solid line), maximum (dash-dot line) and minimum (dotted line) values are given in Figure 4.26. Relative rotor angle $3-1$ has shown for several simulations. Despite the fact that the proposed technique is model based, the damping effectiveness of the proposed controller has been ascertained by nonlinear time simulation. For all the cases the controller performs effectively and damp the 
oscillations as shown in Figure 4.27 with minimum, maximum and mean values. In brief, decentralized adaptive backstepping control is presented and implemented to stabilize multimachine power systems where generator parameters have bounded uncertainties. Test results show the effectiveness of the proposed controller in presence of parameter uncertainties of generators.
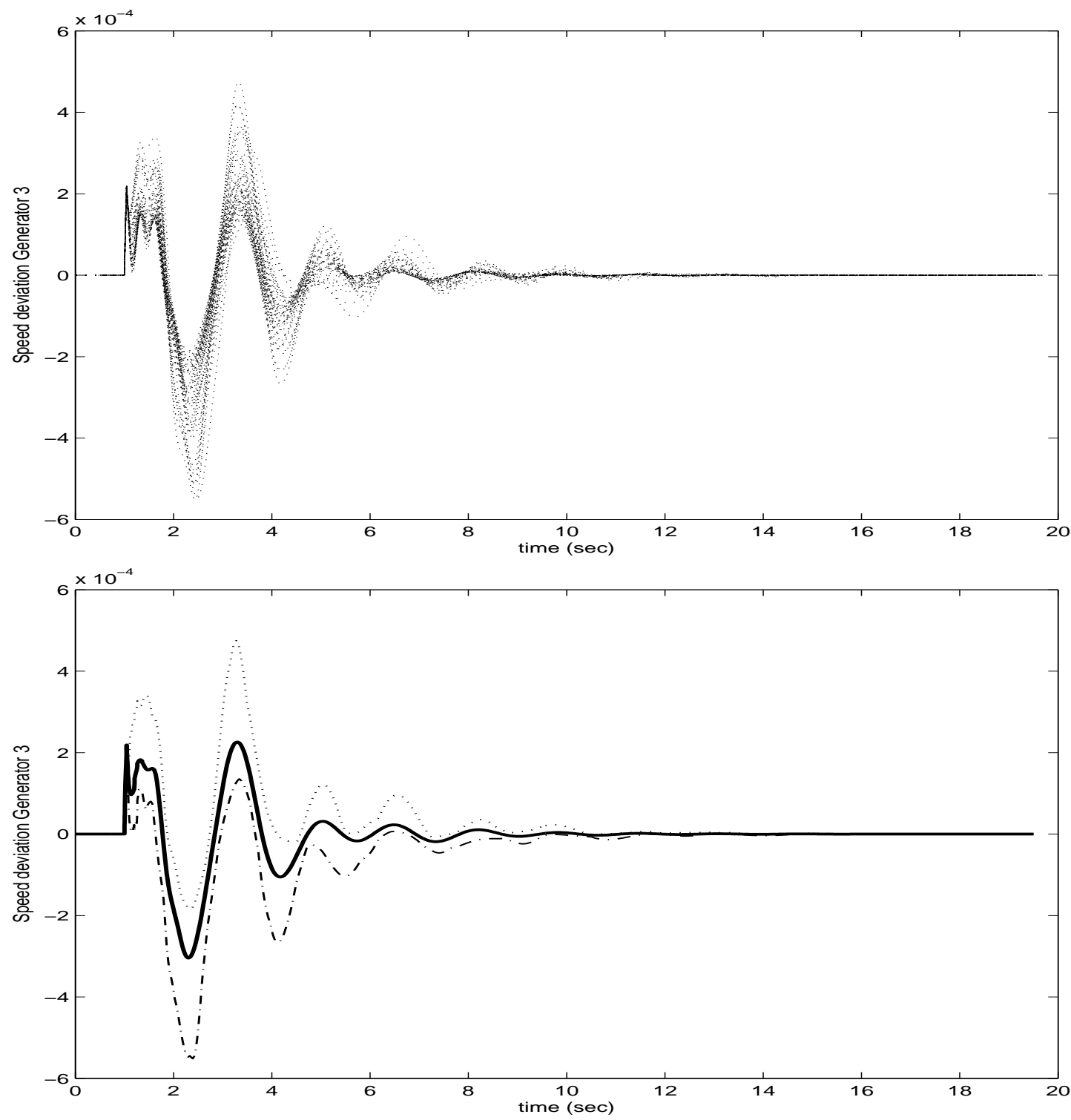

Figure 4.26: Speed deviation $\Delta \omega_{3}$ for several simulations, minimum (dash-dotted line), mean (solid line), and maximum (dotted line) values for $\Delta \omega_{3}$, Scenario-II 

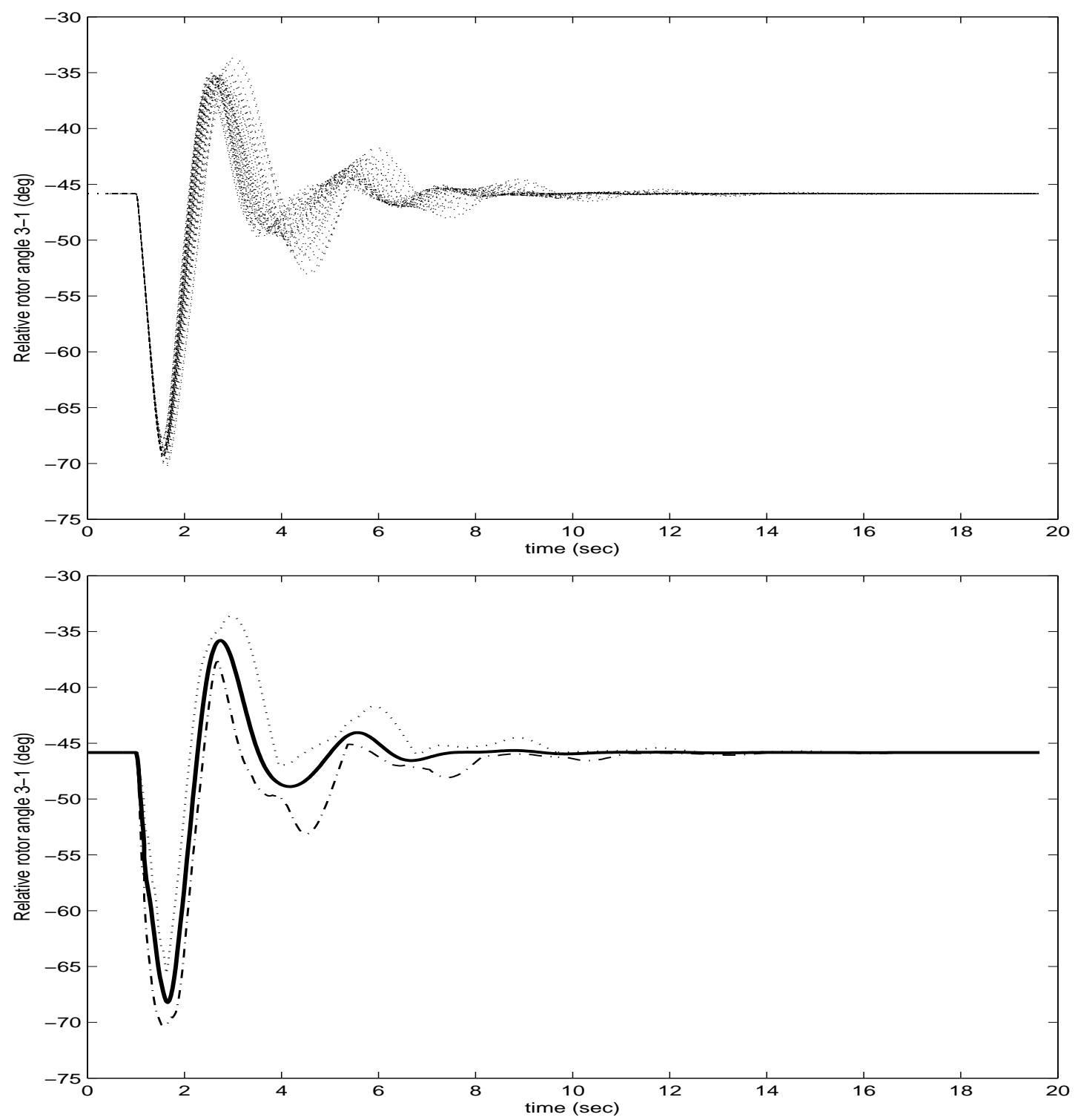

Figure 4.27: Relative rotor angle $\Delta \delta_{31}$ for several simulations, minimum (dash-dotted line), mean (solid line), and maximum (dotted line) values for $\Delta \delta_{31}$, Scenario-II 
The controller which is presented is a model-based control and designed based on transient dynamics of generator. Detailed dynamics of generators (subtransient model) is considered to implement same controllers. For this case four subtransient generators are considered for two area system benchmark. The controllers are implementing on generators 2 and 3 . Comparison is done between the response of controlled system with two different generator dynamics as transients and subtransients. Parameters for the generators are given in appendix 7.3. Direct and quadrature subtransient impedances are denoted by $X_{d}^{\prime \prime}$ and $X_{q}^{\prime \prime}$ respectively. Also time constant for direct and quadrature open circuit are $T_{d o}^{\prime \prime}, T_{q o}^{\prime \prime}$. State equations related to subtransient voltages on direct and quadrature projections are

$$
\begin{aligned}
& \dot{E}_{d}^{\prime \prime}=\left(E_{d}^{\prime}+\left(X_{d}^{\prime}-X_{q}^{\prime \prime}\right) I_{q}-E_{d}^{\prime \prime}\right) \frac{1}{T_{q o}^{\prime \prime}} \\
& \dot{E}_{q}^{\prime \prime}=\left(E_{q}^{\prime}-\left(X_{d}^{\prime}-X_{d}^{\prime \prime}\right) I_{d}-E_{q}^{\prime \prime}\right) \frac{1}{T_{d o}^{\prime \prime}}
\end{aligned}
$$

with algebraic stator equations

$$
\begin{aligned}
& E_{q}^{\prime \prime}-V_{q}=R_{a} I_{q}+X_{d}^{\prime \prime} I_{d} \\
& E_{d}^{\prime \prime}-V_{d}=R_{a} I_{d}-X_{q}^{\prime \prime} I_{q}
\end{aligned}
$$

Direct and quadrature transient voltages, angle, and speed dynamics for transients are given in equations (3.33), (3.34), (3.31), and (3.32) respectively.

Figure 4.28 show that the responses of the both systems (transients and subtransients models) are close to each and the controller shows robust performance due to different generator model. Figures 4.28 and 4.29 compares the relative angles and electric power deviations of generators for same contingency. Three phase fault occurs on line $3-101$ at 1.00 second. The fault is cleared after $45 \mathrm{msec}$ and line is reconnected back at $50 \mathrm{sec}$. Solid line is provided through simulation of transient model and dotted line presents the simulated model with subtransient model. Numerical simulations demonstrate that the proposed controller provides the proper response that behavior of transient and subtransient synchronous generator over a time scale of transient stability are almost the same. Depending on the time frame of stability of power system, different study model for generator can be selected. The results show that the response of the system based on transient and subtransient models have negligible error. 

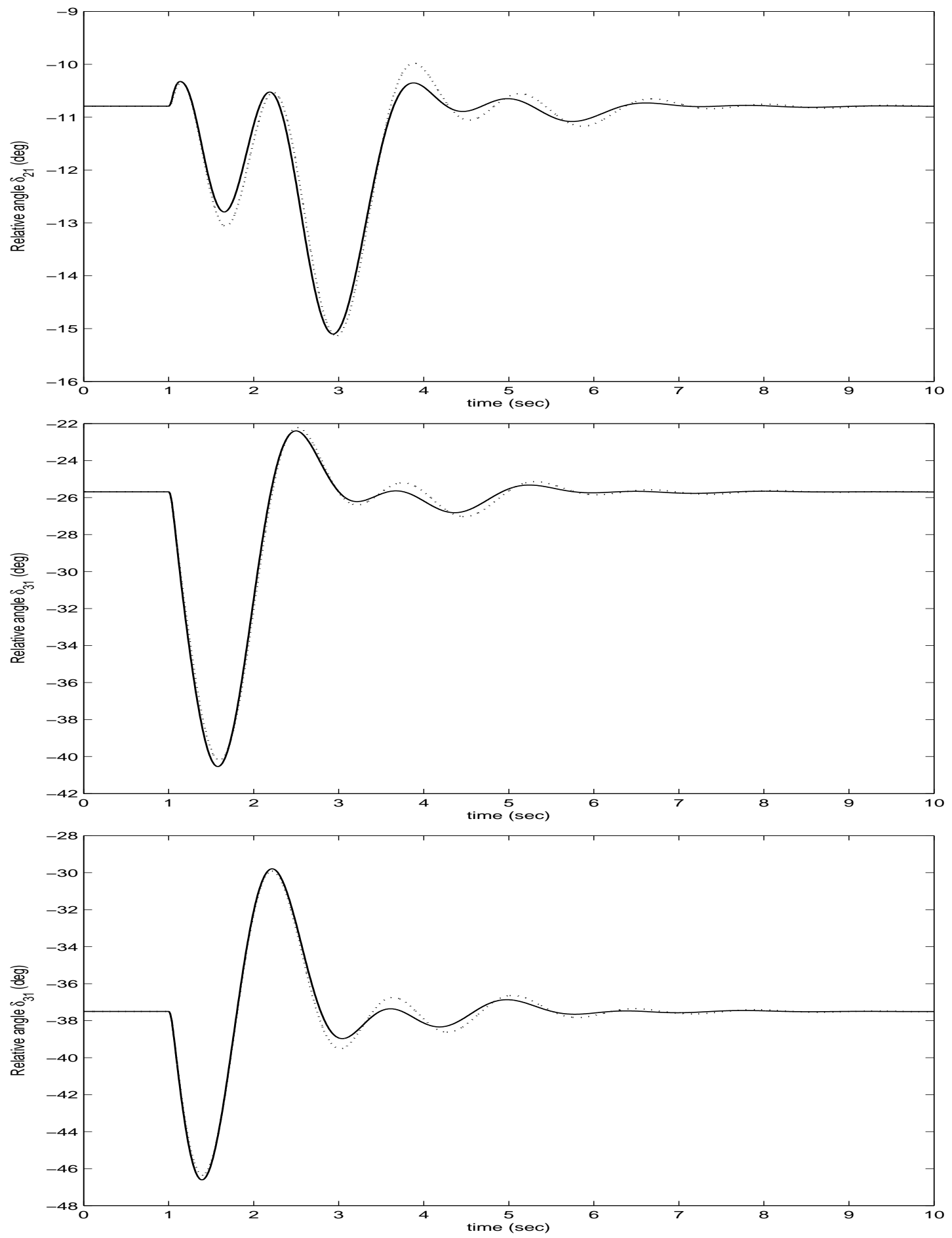

Figure 4.28: Relative angles $\Delta \delta_{21}, \Delta \delta_{31}$, and $\Delta \delta_{41}$. Electric power deviation output of Subtransient generator model (dotted line) and transient model (solid line) 

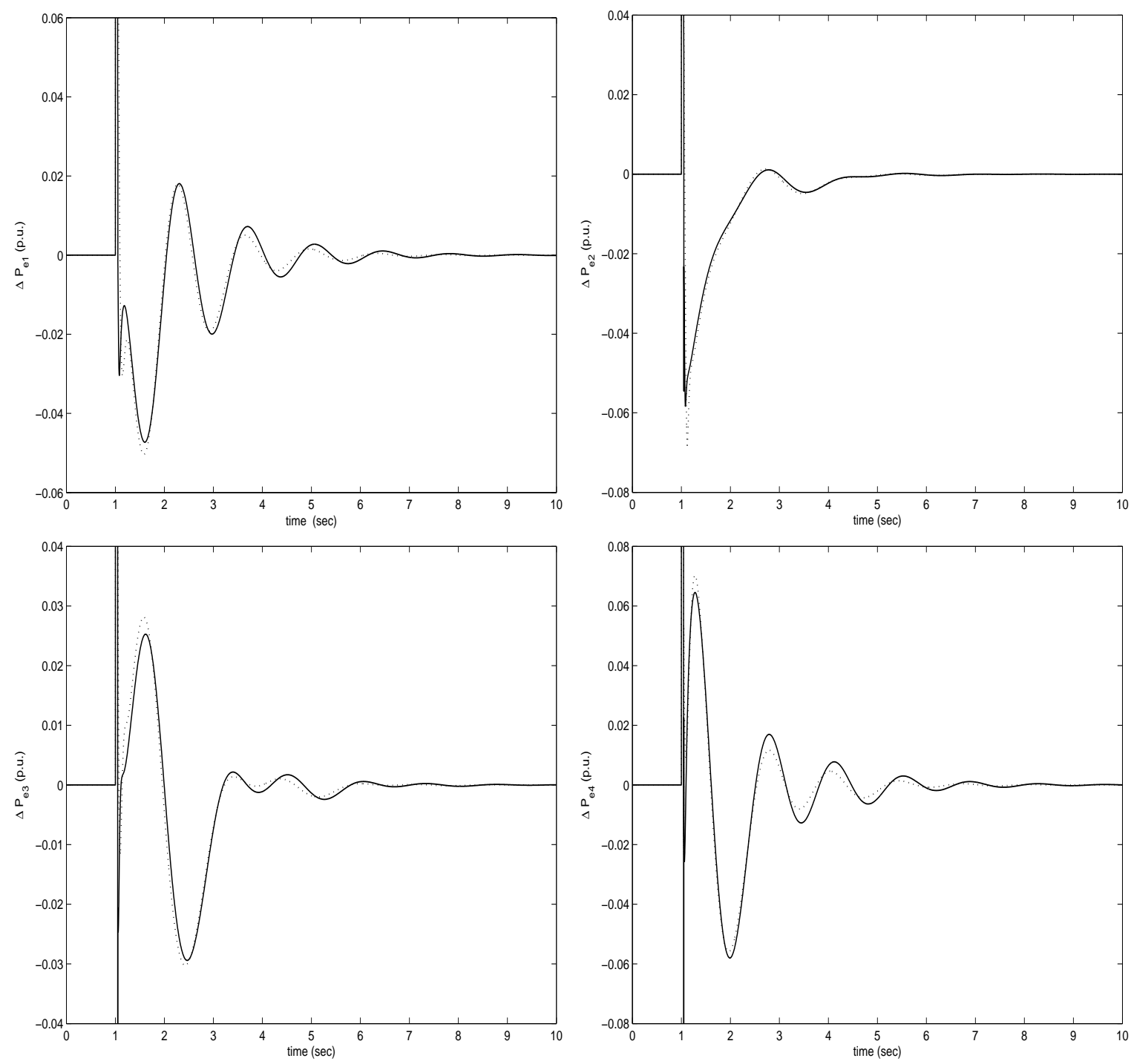

Figure 4.29: Electric power deviations $\Delta P_{e 1}, \Delta P_{e 2}$, and $\Delta P_{e 3}$, and $\Delta P_{e 4}$. Relative angles of Subtransient generator model (dotted line) and transient model (solid line) 


\section{CHAPter}

5

\section{Transient Stability Enhancement Using SVC}

\subsection{Static Var Compensator}

FACTS concept is related to incorporating of power electronic devices into high voltage side of the grid to make it more controllable. Static Var Compensators (SVCs) are shunt FACTS devices which can provide continuous and rapid control of reactive power and voltage, enhancing several aspects of transmission system performance. Including prevention of voltage collapse, enhancement of transient stability, and enhancement of damping of system oscillations. These devices are certainly playing an increasing role in the operation and control of today's power systems. FACTS devices can provide more power transfer with no major changes in the system transmission or generations. It can be placed in transmission and increase power transfer capabilities. In addition, it reduces the operation and transmission investment cost significantly. As mentioned, these devices increase the controllability of power systems but it requires proper damping control technique. Contribution of SVC to the damping of system oscillations resulting from voltage regulation is not significant and usually it requires damping controller. In addition, appropriate damping control requires the designer to have concern about the choice of the device, the most effective measurement to be utilized as input signal, the damping scheme, the control design strategy, and evaluation of the resulting overall system 
performance.

Static var compensators are used to control the voltage at the bus through variable reactive impedance. Because SVC has no rotating part, it is called static. The SVC, type that is used, contains a thyristor switched capacitor bank in parallel with a phase angle controlled reactors. By modifying thyristor firing angle $\alpha$ the current flow through the inductor is adjusted. Firing angle range is $\left[\frac{\pi}{2}, \pi\right]$, that makes SVC reactance changes from inductive to capacitive. The algebraic difference between these two capabilities (capacitive and inductive) is called the dynamic range. Equivalent model for SVC in transient stability study is shown in 5.1. SVC

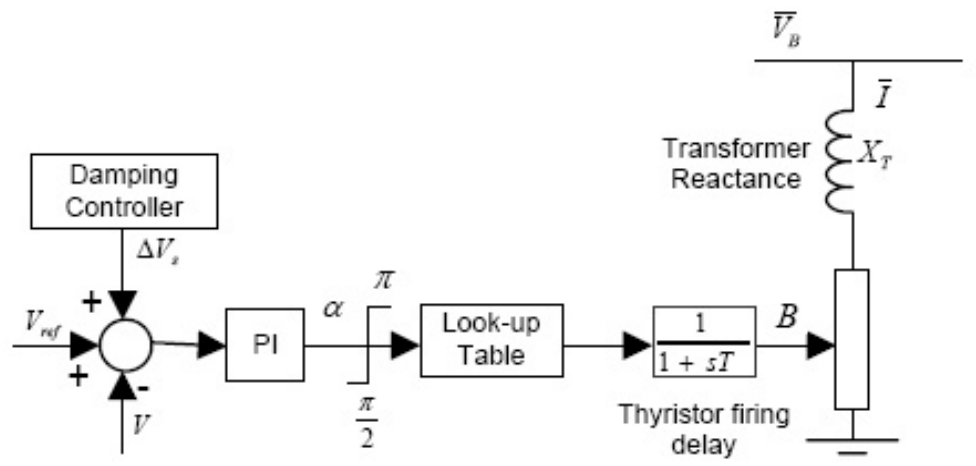

Figure 5.1: SVC Equivalent Model

is used to keep the bus voltage at specific value. By varying its reactive power output in response to the demand of an automatic voltage regulator, it can maintain constant voltage at the point in the system to which it is connected. It can presented as generator bus where injected active power is set to zero and the required reactive power is computed through load flow algorithm. Desired steady state values are obtained as follow

$$
\begin{aligned}
\bar{I} & =\left(\frac{j Q_{B}}{\overline{V_{B}}}\right)^{*} \\
\bar{V} & =\bar{V}_{B}+j X_{T} \bar{I} \\
j B & =-\frac{\bar{I}}{\bar{V}} \\
B(\alpha) & =\frac{2 \alpha-\sin (2 \alpha)-\pi\left(2-\frac{X_{L}}{X_{C}}\right)}{\pi X_{L}}
\end{aligned}
$$

$Q_{B}$ injected reactive power, $\bar{V}_{B}$ bus voltage. $B$ is equivalent $\mathrm{SVC}$ admittance. The firing angle is input to the look up table using $X_{L}, X_{C}$, and $90^{\circ} \leq \alpha \leq 180^{\circ}$. Actual admittance for $\mathrm{SVC}$ is found from pre-computed look up table using $X_{L}, X_{C}$, and $\alpha$ range. Dynamic of the 
thyristor firing delay is approximated by first order block with time delay constant $(T \approx 3 m s)$. $P I$ controller is used to set the $\alpha$ within the limits.

\subsection{SVC Backstepping Damping Nonlinear Controller Design for Power Systems}

\subsubsection{Introduction}

In this section a damping controller for a Static Var Compensator (SVC) is designed using a backstepping controller technique to enhance voltage regulation and power system stability. The damping control design is formulated as an optimization using detailed model of power system including dynamic and static load. A PSO algorithm (3.2) is used to obtain optimal control parameter gains. Nonlinear time domain simulations are carried out to examine the performance of the proposed control technique on a single and multi machine power system. The results for both systems show that the proposed SVC damping controller is superior to conventional lead-lag design especially under high loading conditions and severe contingencies. Much of the effort involved in stability control concerns the avoidance or suppression of oscillations and avoiding cascade failure of the entire power system.

\subsubsection{SVC Control Design}

Static Var Compensators are used in power system to increase transmittable power by regulating the bus voltage and suppressing power oscillations. The main purpose of this device is to control the voltage. Since damping contribution from voltage regulation is not enough, providing a proper damping control signal to voltage control loop is required. Proposed damping controller is based on backstepping technique. Dual input signals are considered for this controller, which are generator speed and electric power deviations from their nominal values, as shown in Figure 5.2. The backstepping controller is nonlinear function of a form: $u=\Phi\left(\Delta \omega, \Delta \delta, \Delta P_{e}, k_{1}, k_{2}, k_{3}\right)$, where $\Delta \omega, \Delta \delta, \Delta P_{e}$ are speed, angle and electric power deviation. $k_{1}, k_{2}, k_{3}$ are control gains. The schematic block diagram with conventional lead-lag controller is shown in Figure 5.2. Electric power deviation is used as the input to the controller. It consists of a filter and lead-lag blocks. Backstepping is a model based controller and is applied to a system whose dynamics are transformed into a specific form. The procedure 

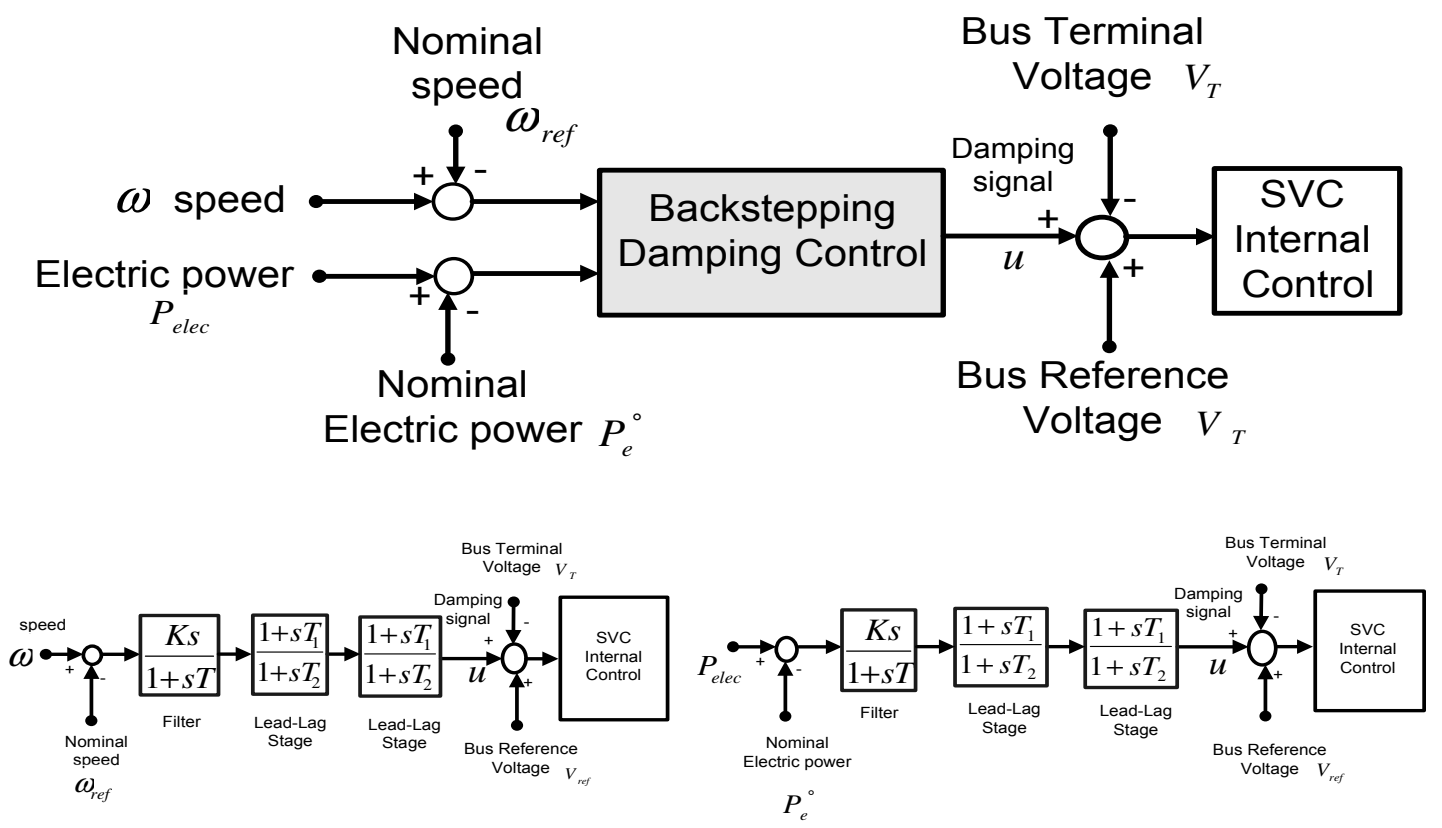

Figure 5.2: SVC damping controllers with nonlinear control structure (backstepping) and Lead-Lags to obtain backstepping control is similar as explained in Section 3.1. In brief for the system of differential equations (5.2)

$$
\begin{aligned}
\dot{x} & =f_{0}+g_{0} z_{1} \\
\dot{z}_{1} & =f_{1}+g_{1} z_{2} \\
\dot{z}_{2} & =f_{2}+g_{2} u
\end{aligned}
$$

The closed form backstepping controller is as follows

$$
u=\frac{1}{g_{2}}\left[-\frac{\partial V_{1}\left(x, z_{1}\right)}{\partial z_{1}} g_{1}-k_{3}\left(z_{2}-\phi_{1}\left(x, z_{1}\right)\right)+\frac{\partial \phi_{1}}{\partial x} \dot{x}_{1}+\frac{\partial \phi_{1}}{\partial z_{1}} \dot{z}_{1}-f_{2}\right]
$$

and Lyapunov function of the system (5.2) is given below.

$$
V_{2}\left(x, z_{1}, z_{2}\right)=\frac{1}{2} x^{2}+\frac{1}{2}\left[z_{1}-\phi_{0}(x)\right]^{2}+\frac{1}{2}\left[z_{2}-\phi_{2}\left(x, z_{1}\right)\right]^{2}
$$

Controller $u$ provides signal that stabilizes the dynamics of $x, z_{1}, z_{2}$. Note that controller gains $k_{1}, k_{2}, k_{3}$ require tuning to obtain optimal performance of the closed loop system. 


\subsubsection{Optimal Settings of SVC Control Gains}

The task of PSO (as explained in Section 3.2) is to search for an optimal set of gains for SVC to regulate the voltage and damp the power oscillations. The objective function $J$ to be optimized by PSO is therefore formulated as a weighted quadratic sum of generator speed and bus voltage deviations from their nominal values.

$$
\min _{\text {Find } K_{i}} J=\int_{0}^{t}\left[\alpha(\Delta \omega)^{2}+\gamma\left(\Delta V_{b u s}\right)\right] d t
$$

PSO method for obtaining the damping controller gain is implemented without linearizing the system. The technique is implemented on a single and a multi machine power system.

\subsubsection{Single Machine Infinite Bus}

A single machine infinite bus system with AC transmission link is analyzed here [65]. The system is presented by nonlinear differential and algebraic equations including synchronous generator, load, SVC with damping controller and very large AC network represented by an infinite bus. Parameters for generator and line reactance are given in Table 5.1. SVC is connected at the load bus that is separated from generator bus through a transformer and an additional line reactance.

Inputs to the damping controller of SVC are speed and electric power deviation respectively $\Delta \omega=\omega-\omega_{r e f}, \Delta P_{e}=P_{e}-P_{e}^{\circ}$. Hence it is needed to bring the generator speed deviation back to zero [53]. Dynamic equations related to generator are:

$$
\begin{aligned}
\Delta \dot{\delta} & =\Delta \omega \\
\Delta \dot{\omega} & =-\frac{D}{2 H} \Delta \omega-\frac{\omega_{r e f}}{2 H} \Delta P_{e} \\
\Delta \dot{P}_{e} & =\xi+\beta u
\end{aligned}
$$

Where $\xi$ and $\beta$ are functions defined below and $u$ is a damping control signal.

$$
\begin{aligned}
\xi & =-\frac{\Delta P_{e}}{\tilde{T}_{d}}+\frac{x_{q}-x_{d}}{x_{q \sum} \tilde{T}_{d}}+P_{e} \omega \cot \delta-\frac{P_{e} \circ}{\tilde{T}_{d}}+\frac{V_{b_{u s_{\infty}}} \sin \delta}{x_{q \sum} \tilde{T}_{d}} \\
\beta & =\frac{V_{b u s} \sin \delta}{x_{q \sum}} \tilde{T}_{d}
\end{aligned}
$$

Equation 5.7 is in strict feedback form, so backstepping controller can be applied. Note that SVC is basically a shunt connected device whose output is adjusted to exchange capacitive 


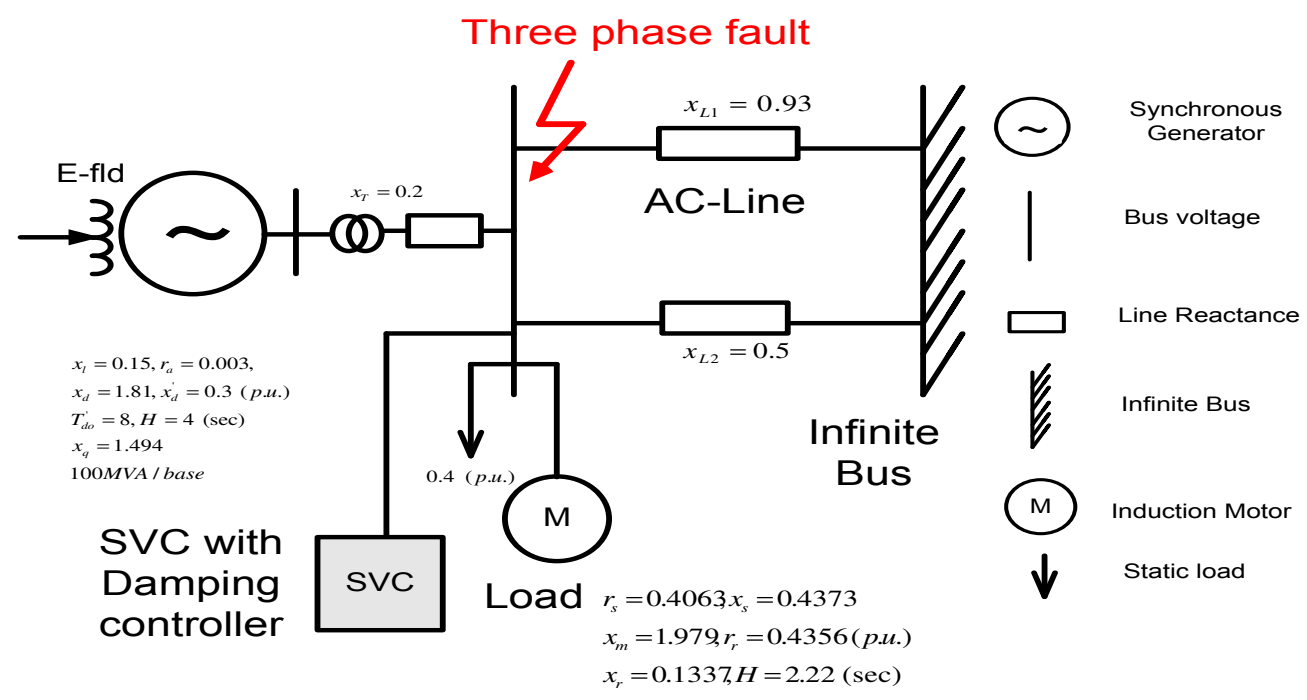

Table 5.1: Analyzed Scenarios

\begin{tabular}{c|c}
\hline Leakage reactance (p.u.) & $x_{l}=0.15$ \\
\hline Resistance (p.u.) & $r_{a}=0.003$ \\
\hline d-axis reactance (p.u.) & $x_{d}=1.81$ \\
\hline d-axis transient reactance (p.u.) & $x_{d}^{\prime}=0.3$ \\
\hline d-axis open circuit time constant (sec) & $T_{d o}^{\prime}=8$ \\
\hline q-axis reactance (p.u.) & $x_{q}=1.494$ \\
\hline Inertia Constant (sec) & $H=4$ \\
\hline Reactance AC line (p.u.) & $x_{L_{1}}=0.93$ \\
\hline Reactance AC line (p.u.) & $x_{L_{2}}=0.5$ \\
\hline Transformer+line reactance & $x_{T}=0.2$ \\
\hline$x_{d \sum}^{\prime}$ & $x_{L}+x_{d}^{\prime}+x_{T}$ \\
\hline$x_{q \sum}^{\prime}$ & $x_{L}+x_{q}^{\prime}+x_{T}$ \\
\hline$\tilde{T}_{d}$ & $T_{d o}^{\prime} \frac{x_{d \Sigma}^{\prime}}{x_{q \Sigma}}$ \\
\hline
\end{tabular}


or inductive current in order to maintain and control specific power system variables. Active power equation 5.8 with SVC installed is obtained as:

$$
P_{e}=\frac{E_{q}^{\prime} V_{b u s \infty}}{x_{d \sum}^{\prime}-x_{L} B_{s v c}\left(x_{d}^{\prime}+x_{T}\right)}
$$

where $x_{d \sum}=x_{L}+x_{d}+x_{T}, B_{S V C}$ is SVC susceptance, $x_{L}=x_{L 1}+x_{L 2}$, and $V_{b u s_{\infty}}$ is the infinite bus voltage [65], [38]. Static and dynamic loads are both connected at the load bus. Static load is represented as a function of bus voltage. Induction motor represented the dynamic load with the following parameters in per unit:

Stator resistance $r_{s}=0.4063$

Stator leakage reactance $x_{s}=0.4373$

Magnetizing reactance $x_{m}=1.9979$

Rotor resistance $r_{r}=0.4356$

Rotor leakage reactance $x_{r}=0.1337$

Inertia constant $H=2.224$ (sec)

Following scenarios are presented here for illustrating the performance of the proposed controller.

- Effect of fault duration

- Effect of dynamic load (induction motor)

- Effect of low to high power generation

Effect of fault duration: The first scenario demonstrates the effect of fault duration on SVC control performance. Proposed damping controller with backstepping is compared with a lead-lag controller. PSO algorithm is used for tuning controller gains with defined objective function 5.5. Tuning process is performed during a three phase fault on transmission line $2-3$ (Figure 5.1). The fault is cleared by removing the line after $35 \mathrm{msec}$. The line is reconnected after 60 msec. Table 5.2 shows the tuned controller gains. By increasing the fault duration lead-lag damping controller cannot damp the oscillations effectively in comparison with proposed backstepping controller. Figure 5.4 shows the relative angle of generator. For longer fault duration (66 msec), it becomes subtle to achieve the stability of closed loop system. Lead-lag damping controller can no longer stabilize the system while the proposed controller does. 
Table 5.2: Damping Controller Gains

\begin{tabular}{c|c}
\hline Lead-Lag Damping Controller Gains & Backstepping Damping Controller Gains \\
\hline$K=80.1749$ & $k_{1}=1$ \\
\hline$T=6.4087$ & $k_{2}=9.0768$ \\
\hline$T_{1}=0.1687$ & $k_{3}=0.42287$ \\
\hline$T_{2}=0.4753$ & \\
\hline
\end{tabular}

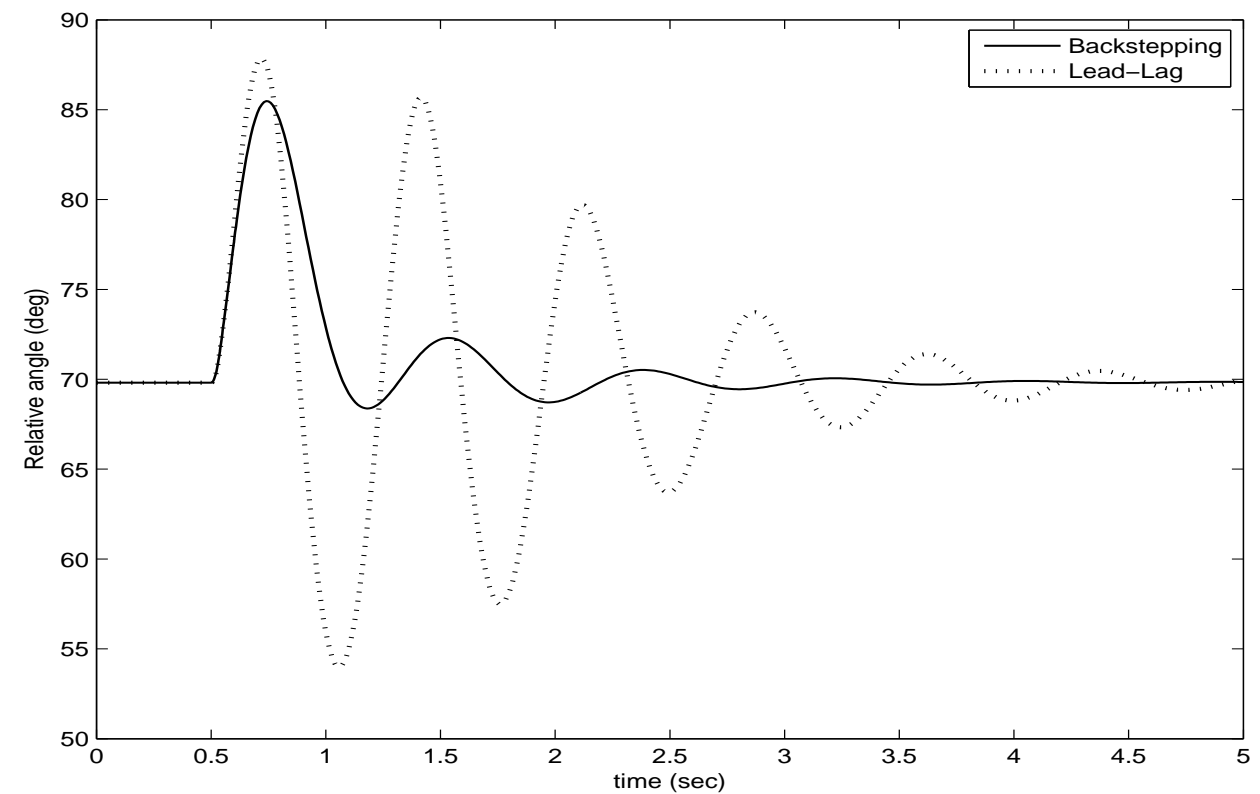

Figure 5.3: $\quad$ SVC with lead-lag damping controller (dotted line :),SVC with backstepping damping controller (solid line -). Fault duration $40 \mathrm{msec}$

Effect of Dynamic load (induction motor): Dynamic load in this study is presented by an induction motor. A three phase fault scenario away from nominal operation of system has a $42 \mathrm{msec}$ fault duration. Response of the proposed controller is compared with a lead-lag controller. Terminal voltage and relative angle of generator are improved as shown in Figures 5.5. The simulation results show that nature of load can affect the transient behavior of the system. Induction motor with MVA base $=75$ and inertia $H=8.22$ (sec) is included, the model is obtained from [13]. Parameters of induction motor in per unit are:

Stator resistance $r_{s}=0.0041$

Stator leakage reactance $x_{s}=0.04373$

Magnetizing reactance $x_{m}=1.9799$ 


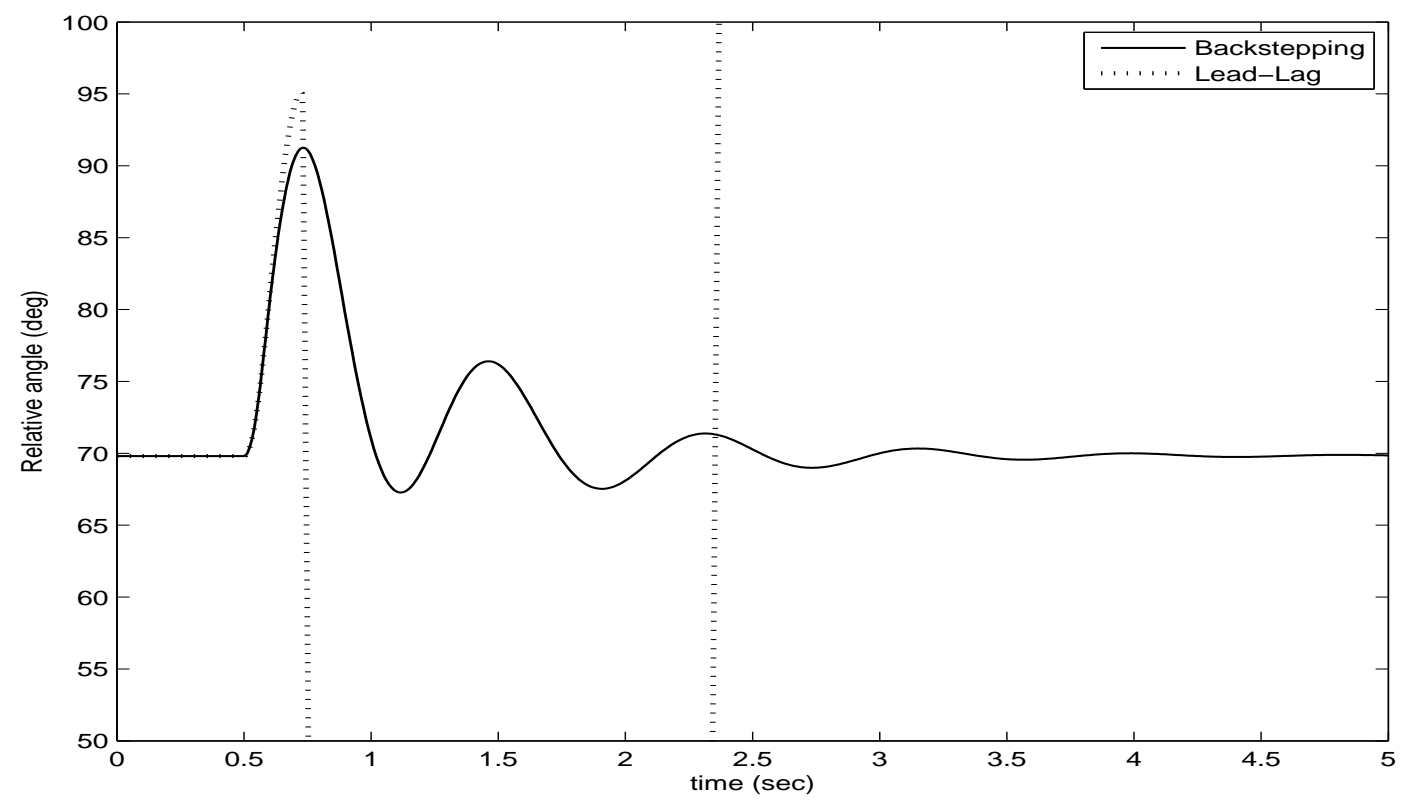

Figure 5.4: SVC with lead-lag damping controller (dotted line :),SVC with backstepping damping controller (solid line -). Fault duration $66 \mathrm{msec}$
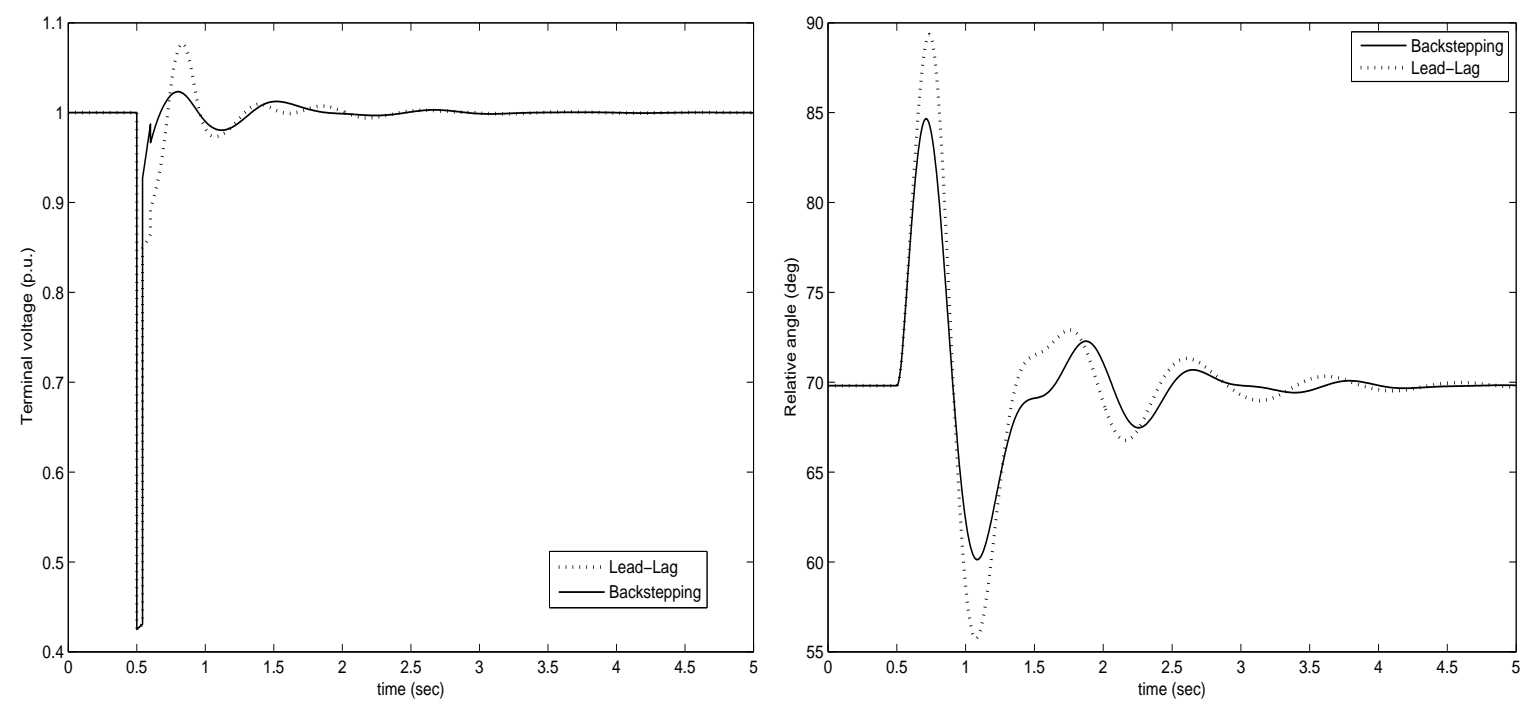

Figure 5.5: Terminal voltage under a large induction load and generator relative rotor angle (deg)

Rotor resistance $r_{r}=0.0044$

Rotor leakage reactance $x_{r}=0.1337$

Effect of low to high generation: In this scenario effect of low power generation to high power generation is investigated. For each set of power generation load flow solution is obtained for each bus. Figure 5.6 demonstrate power and speed deviations. Figure 5.6 shows the effect of 

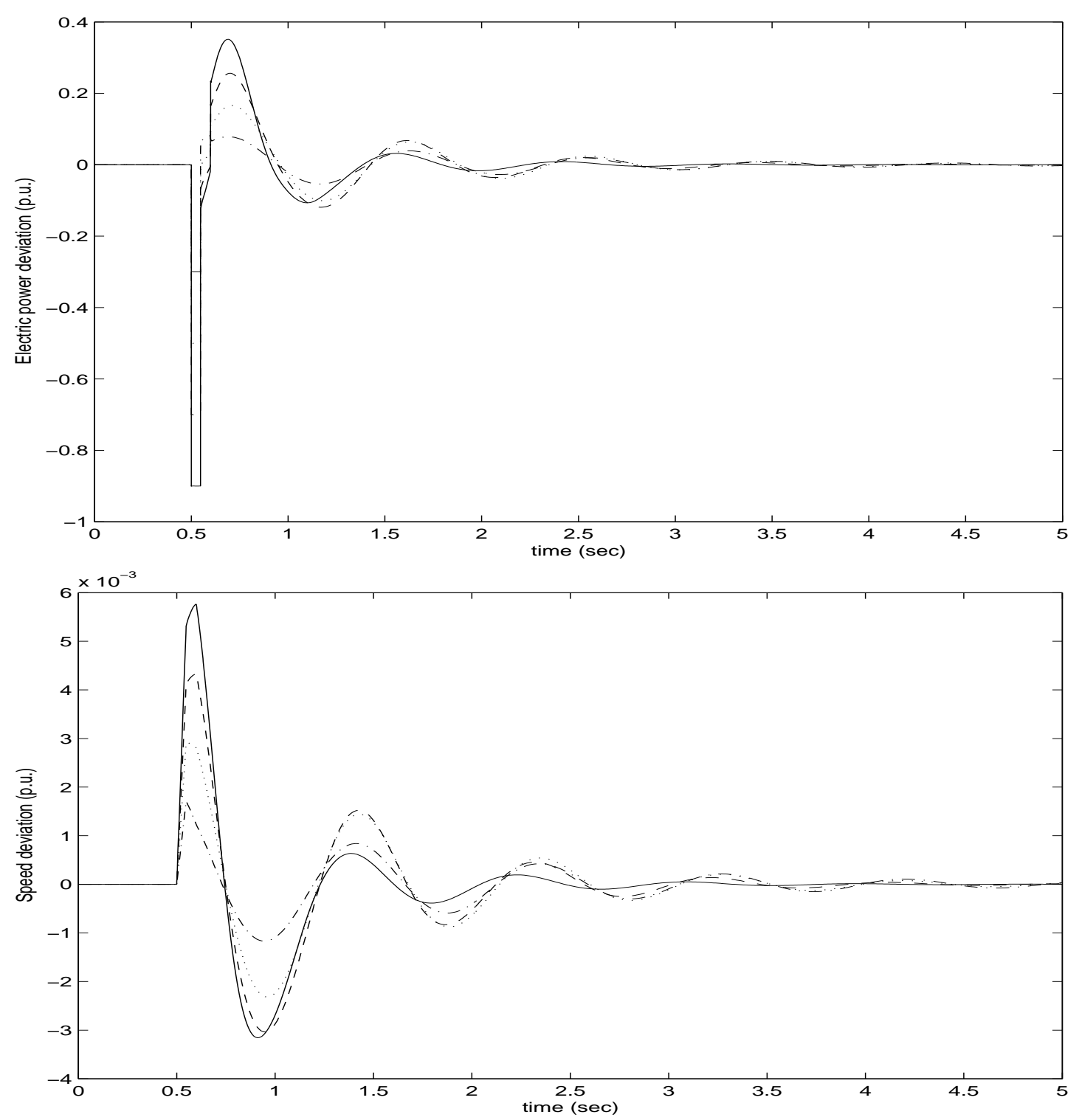

Figure 5.6: Effect of low to high generation on electric power of generator and speed deviation of generator 
low to high power generation on performance of SVC damping controller with backstepping technique. Performance of controller with power generation $P_{G}=0.9$ (p.u.) (solid line), power generation $P_{G}=0.7$ (dash line), power generation $P_{G}=0.5$ (p.u.) (dot line), power generation $P_{G}=0.3$ (p.u.) (dash-dot line).

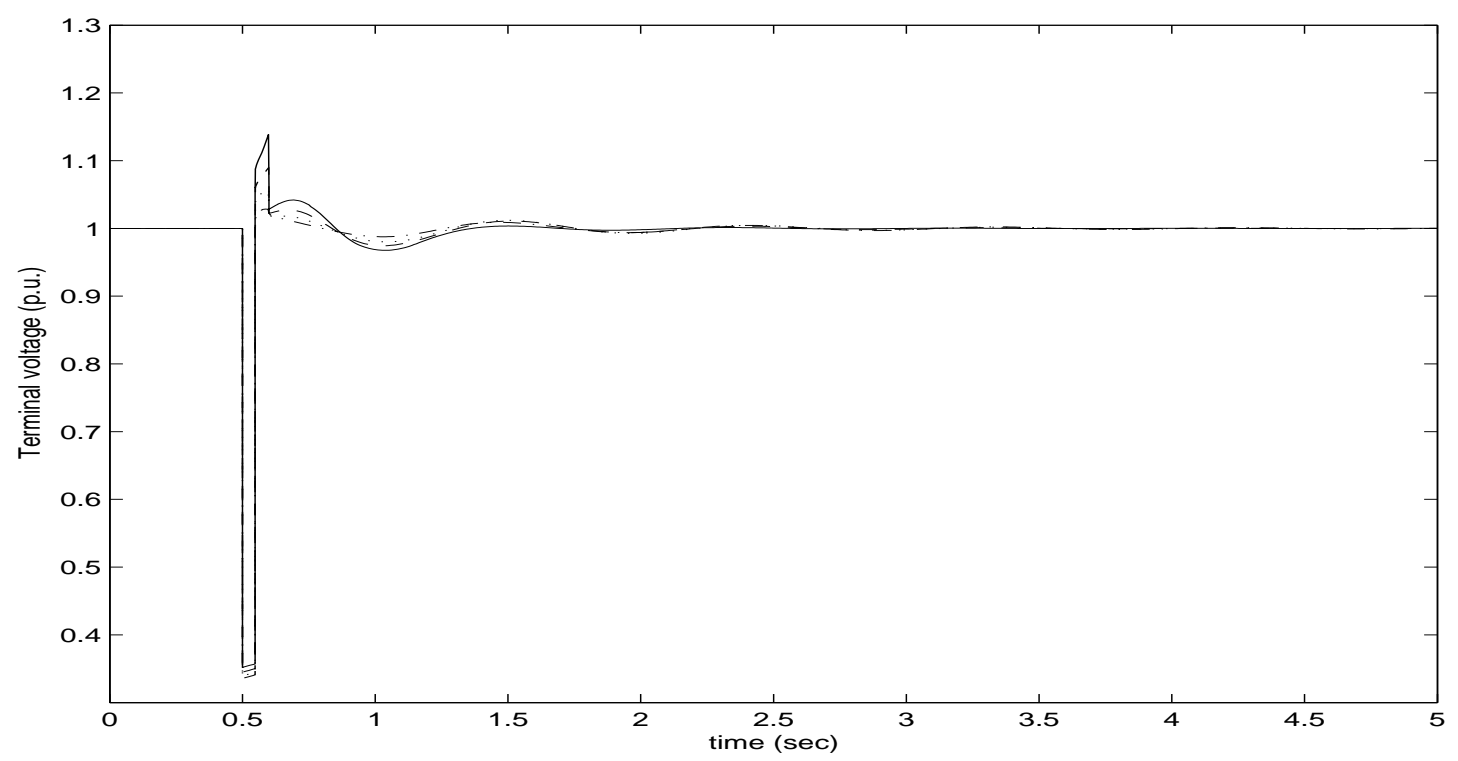

Figure 5.7: Effect of low to high generation power on terminal voltage of generator

\subsubsection{Two Area System}

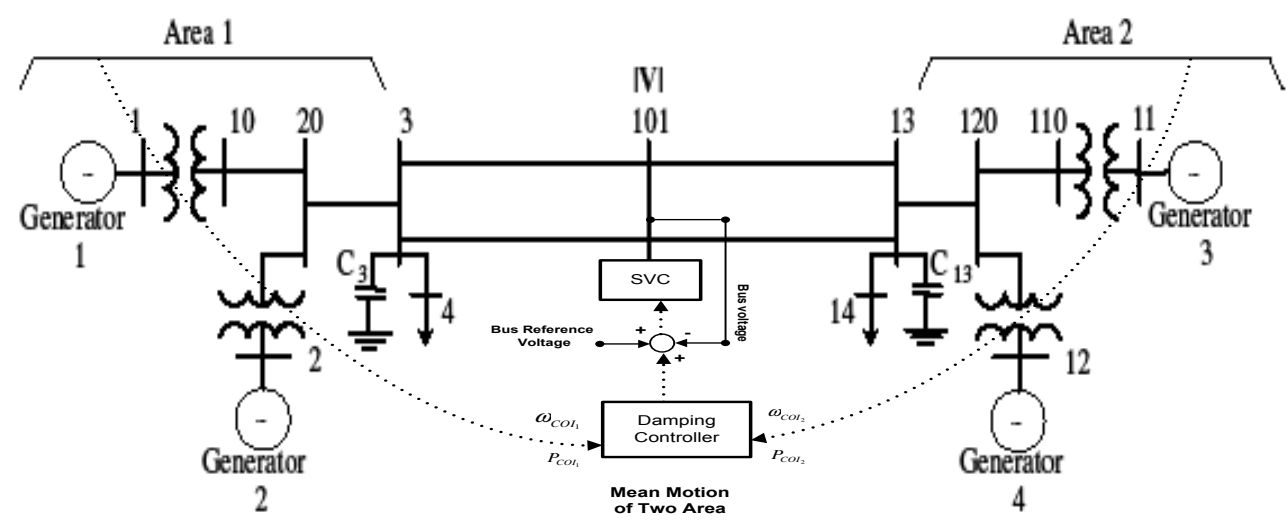

Figure 5.8: Two Area system 
A two area system is considered for this study that is presented as a benchmark for interarea oscillation studies. The system consists of two generators in each area connected via two tie lines. Generators parameters are given in [65]. The effect of SVC on bus 101 has been studied on this system especially with lead-lag damping controller [37]. Schematic block diagram is shown in Figure 5.8. Backstepping technique is used to obtain damping signal to SVC at mid point of transmission line at bus 101. SVC supplies the reactive power at midpoint of long transmission line. To obtain proper damping signal, the controller does not require detailed information from dynamic of each generator. Instead it is possible to design backstepping control strategy based on system center of inertia (COI) trajectory. Objective of controller is to drive the COI's of each area ( 1 and 2$)$ to its equilibrium point. Since COI presents a mean motion of each area, the information is very effective to control inter area oscillation and enhancing the stability of interconnected two area system. More specifically for this system the backstepping control law could keep synchronization of the two interconnected system by driving their dynamics COIs to a stable equilibrium point.

Consider Area-i with $n$ number of generators, center of inertia reference transformation is defined by COI angle, speed, and electric power as:

$$
\begin{aligned}
\delta_{C O I_{i}} & =\frac{1}{M_{T}} \sum_{j=1}^{n} M_{j} \delta_{j} \\
\omega_{C O I_{i}} & =\frac{1}{M_{T}} \sum_{j=1}^{n} M_{j} \omega_{j} \\
P_{e C O I_{i}} & =\frac{1}{M_{T}} \sum_{j=1}^{n} M_{j} P_{e j}
\end{aligned}
$$

where

$$
\begin{aligned}
M_{T} & =\sum_{j=1}^{n} M_{j} \\
M_{i} & =\frac{2 H_{i}}{\omega_{r e f}}
\end{aligned}
$$

Dynamics of relative motion between two areas are formulated with state presented by $\Delta \delta_{C O I_{12}}$, $\Delta \omega_{C O I_{12}}, \Delta P_{e C O I_{12}}$ where:

$$
\begin{aligned}
\Delta \delta_{C O I_{12}} & =\delta_{C O I_{1}}-\delta_{C O I_{2}} \\
\Delta \omega_{C O I_{12}} & =\omega_{C O I_{1}}-\omega_{C O I_{2}} \\
\Delta P_{C O I_{12}} & =P_{C O I_{1}}-P_{C O I_{2}}
\end{aligned}
$$


The derivatives of equations 6.9 can be presented in strict feedback form similar to equation 5.2. A backstepping technique is applied to produce damping signal to SVC. The ability of system to withstand a three phase fault on line $3-101$ in Figure 5.8 is tested. The fault occurred at 0.5 seconds and is cleared after $50 \mathrm{msec}$ by removing the transmission line. The line is reconnected back at 0.67 sec. Proposed SVC damping controller with backstepping is compared with lead-lag that has been designed for this system [37]. Following control strategies are analyzed:

- Proposed backstepping damping controller using COI information

- Lead-Lag damping controller using local information (power deviation at bus 101)

- Lead-Lag damping controller using remote speed measurements from area 1 and 2.

Schematic block for two Lead-Lag damping controller is given in Figure 5.9. Backstepping

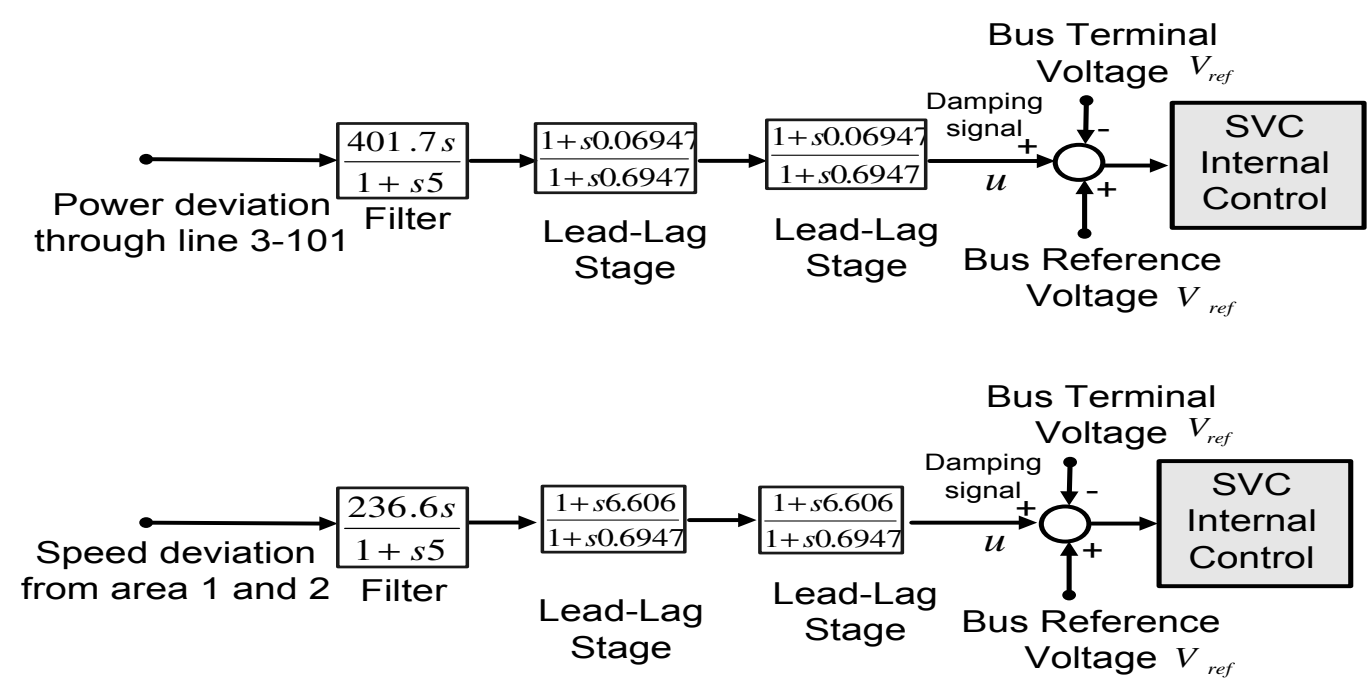

Figure 5.9: Lead-Lag damping signal with electric power and speed inputs

controller gains are tuned with PSO algorithm to optimize the following objective function (Table 5.3).

$$
\min _{\text {Find } K_{i}} J_{1}=\int_{0}^{t} \alpha\left(\Delta P_{C O I_{12}}\right)^{2} d t
$$



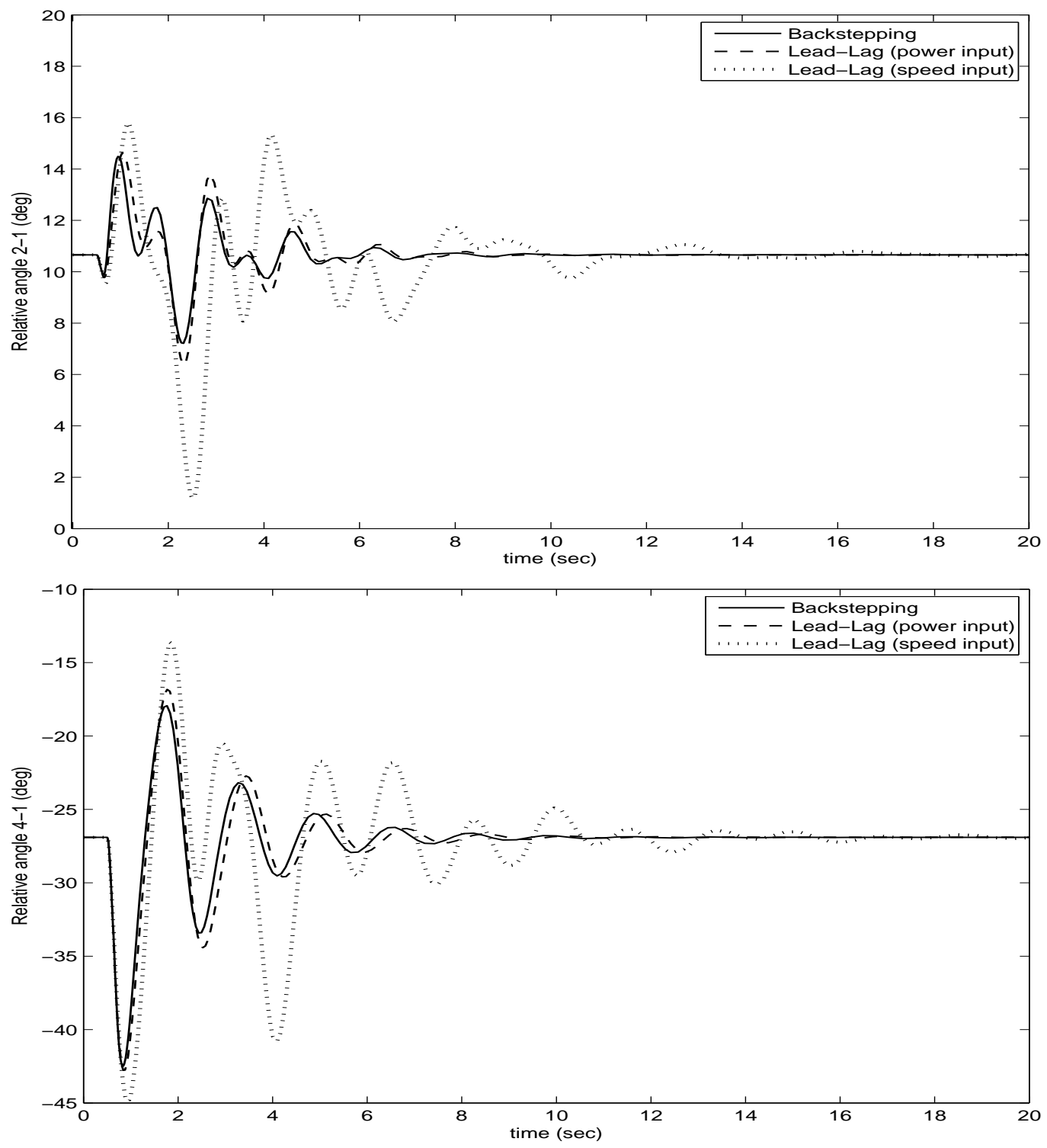

Figure 5.10: Relative angle $2-1$ and $4-1$ (deg)

Figures 5.10 show the comparison between relative rotor angles $2-1,4-1$. Proposed SVC damping controller outperforms two Lead-Lag controllers. With remote information of dynamic COI, the performance of the designed controller is superior to the conventional ones. It should also be noted that with fast development of wide area measurements and control, it is possible to design effective controllers which requires information based on system center of inertia trajectory. In designing a backstepping controller for an SVC the following three different objective functions have been investigated. The results are tabulated in Table 5.3. 
Table 5.3: Backstepping Controller Gains

\begin{tabular}{c|c}
\hline Backstepping Controller Gains & Objective Functions and Weighting Factors \\
\hline$k_{1}=1.9212$ & $J_{1}=\int_{0}^{t} \alpha\left(\Delta P_{C O I_{12}}\right)^{2} d t$ \\
$k_{2}=3.7788$ & \\
$k_{3}=2.1791$ & \\
\hline$k_{1}=1.0312$ & $J_{2}=\int_{0}^{t} \alpha\left(\Delta \omega_{C O I_{12}}\right)^{2}+\alpha\left(\Delta V_{\text {bus }}\right)^{2} d t$ \\
$k_{2}=2.8012$ & \\
$k_{3}=4.228$ & \\
\hline$k_{1}=3.5465$ & $J_{3}=\int_{0}^{t}\left(\Delta P_{\text {tie }}\right)^{2}+\alpha\left(\Delta V_{\text {bus }}\right)^{2} d t$ \\
$k_{2}=3.9490$ & \\
$k_{3}=1.9467$ & \\
\hline
\end{tabular}

The objective function used in the control design can have an impact upon the effectiveness of the controller when it is applied to the system. Speed and terminal voltage for generators 2 are shown in Figure 5.11 under three phase fault condition.

Objective function $J_{1}$ has information related to electric power deviation of each area based on center of inertia calculation. Second objective function $J_{2}$ comprises of speed deviation of each area based on center of inertia and voltage deviation at the bus where the SVC is located. Both of them $J_{1}$ and $J_{2}$ require remote information from each areas to achieve global optimization through PSO algorithm. Instead of using remote signals in optimization process, global signals can also be used instead. For objective function $J_{3}$ local information at the bus is used where the SVC is connected. Response of the backstepping controlled is compared through these three objective function. $J_{1}$ is the better choice to damp the oscillations of the system through SVC damping controller in comparison with other two performance indices as computed as $J_{2}$ and $J_{3}$.

As a result damping controller design for backstepping is formulated as an optimization problem with PSO algorithm. COI information of the system can improve the performance of the system. There is a complementary need to provide large scale feedback controllers with remote status information from each area, so that they can better accommodate critical conditions or events especially those involving major changes in power system structure. Results show that the proposed controller is superior in damping performance to conventional 

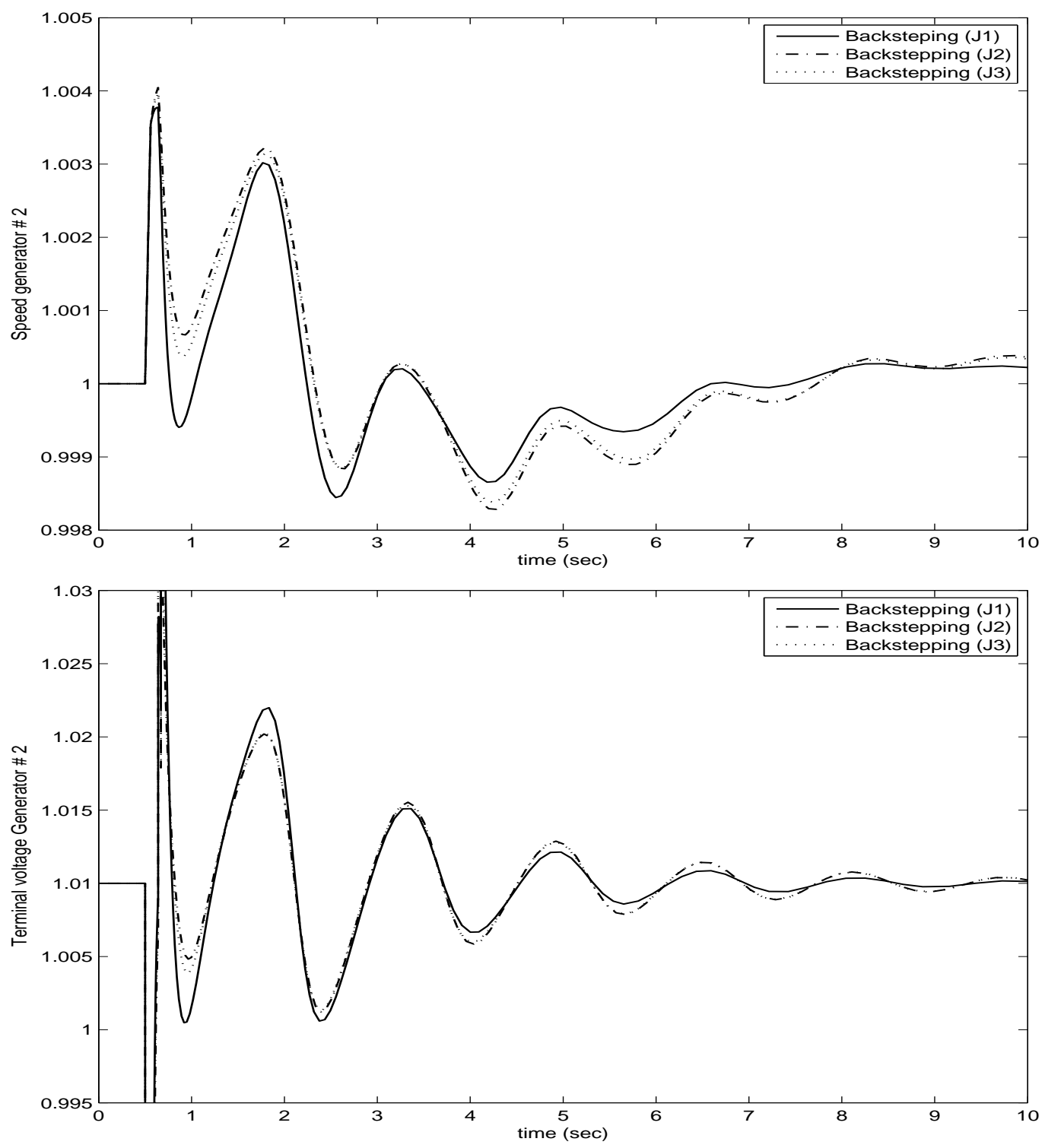

Figure 5.11: Speed and terminal voltage for Generator 2 
controller. During the simulation it is assumed that the wide area signals are available for the controller, the effect of time delay has already been involved in this study.

With rapid advances in wide area measurements technology, the transmission of measured signals from remote generators to centralized control has become relatively simple. Depending on the distance of measurement site, time delay typically in the range of $(0.02-1)$ seconds is expected and incorporated in simulation results [83], [17]. The controller performed satisfactorily in different time delay within the specified delay time. Two different values for time delay 0.04 seconds (dash-dotted line) and 0.09 seconds (dotted line) are considered for the following scenario. Solid line denotes the relative angles $\delta_{21}, \delta_{31}$, and $\delta_{41}$ with no time delay. Transmission line is removed at 1.50 second and reconnected back after 0.27 sec. In this study a fixed time delay has been considered for all communication channels through the control inputs. In more realistic case, this might not be the case as the distance of measurement areas differs from the control center. The dynamic response of the system following the large disturbance (Figures 5.12, 5.13) show that the oscillations are damped in short period of time, and the COI feedback signals arrive at the control location at bus 101 after a finite time delay. For optimization of centralized controller gains, performance index $J_{1}$ (Table 5.3) is used.

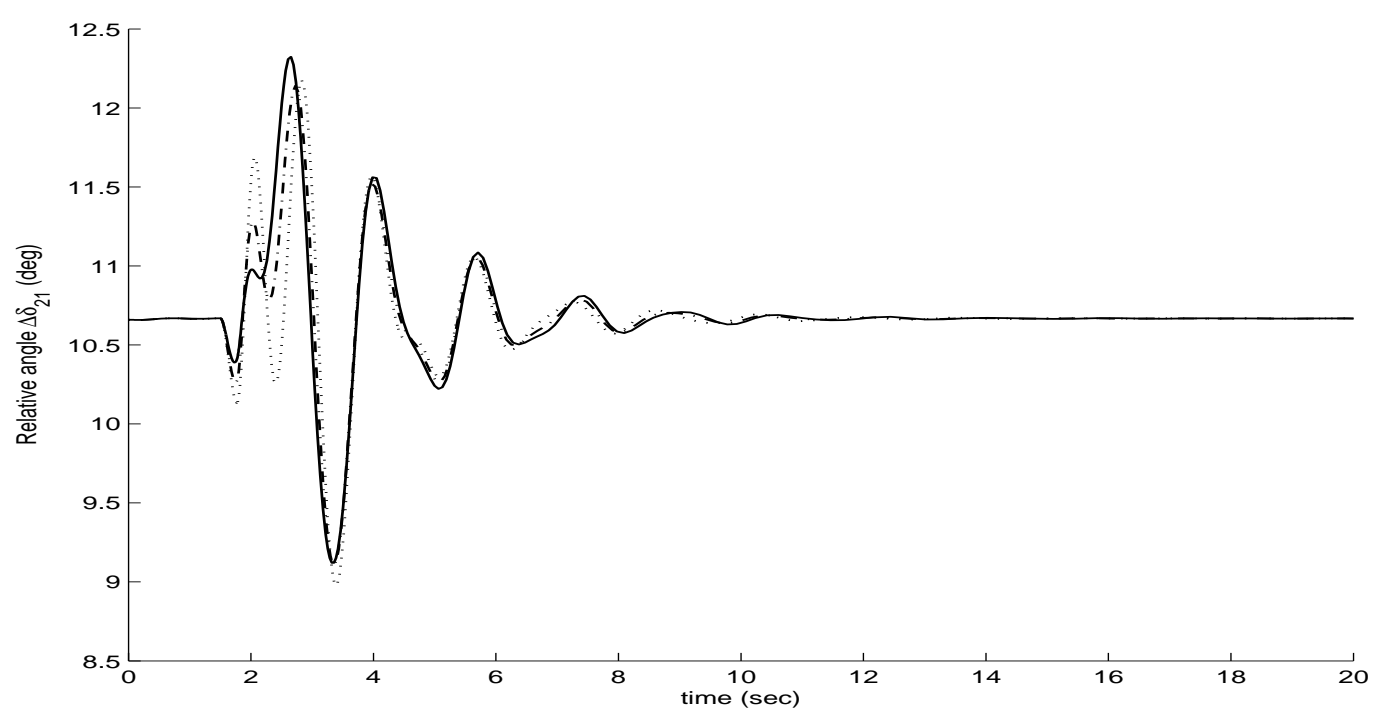

Figure 5.12: Effect of time delay in relative angle $\delta_{21}$. Solid line (no delay), dash-dotted line (delay $0.04 \mathrm{sec}$ ), dotted line (delay $0.09 \mathrm{sec}$ ) 

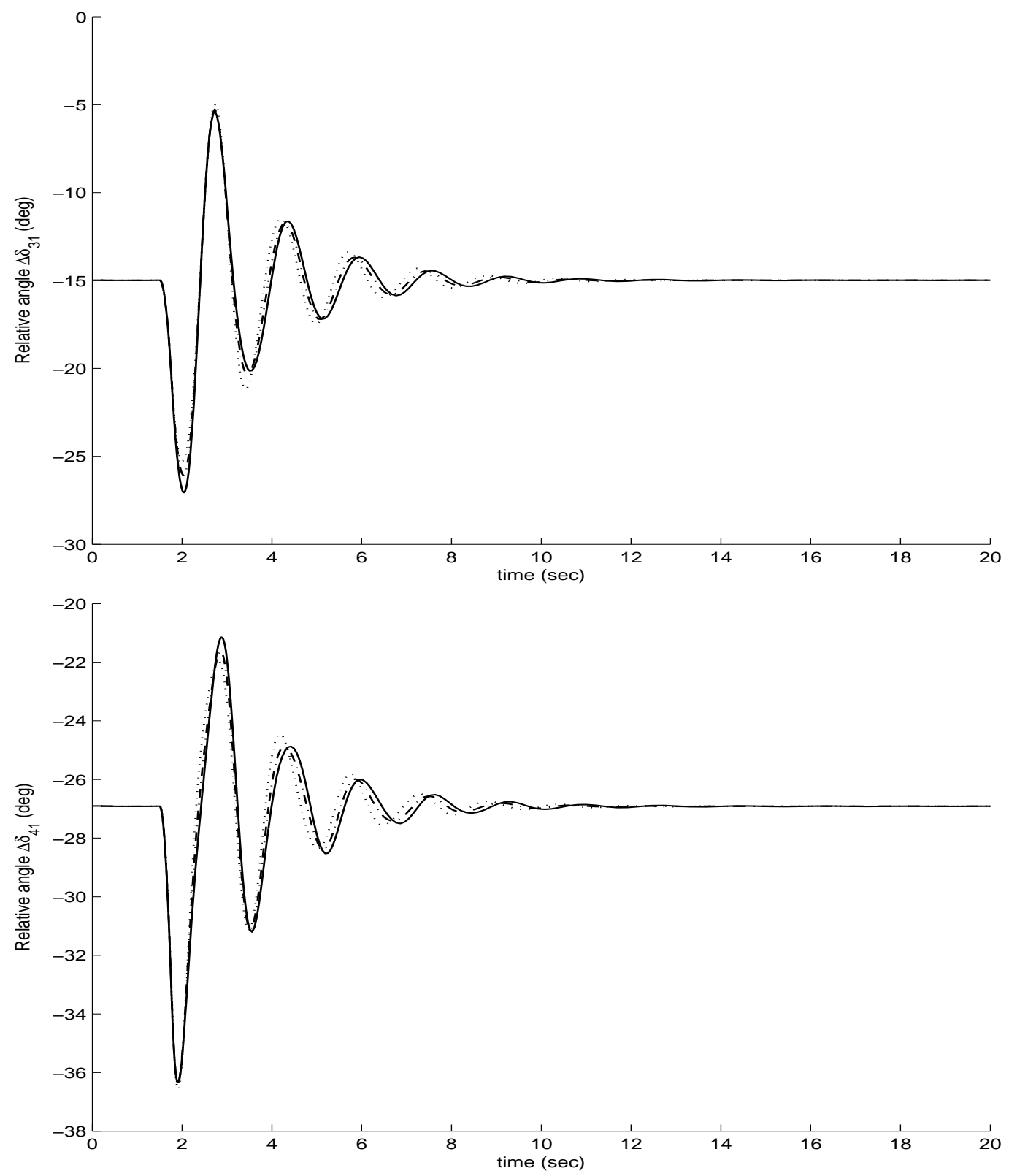

Figure 5.13: Effect of time delay in relative angles $\delta_{31}, \delta_{41}$. Solid line (no delay), dash-dotted line (delay $0.04 \mathrm{sec}$ ), dotted line (delay $0.09 \mathrm{sec}$ ) 
Comparison between SVC and excitation system with backstepping damping controllers are considered in following. Fault scenario is a three phase fault at 0.5 sec on line $3-101$. The fault is cleared at $0.57 \mathrm{sec}$ and the line is reconnected back at $0.6 \mathrm{sec}$. The objective function for tuning the gains is $J_{1}$ that is given in Table 5.3. Figur 5.14 compares the relative rotor angles $\delta_{31}$, and $\delta_{41}$. Excitation controller with backstepping provides better damping in comparison with SVC.
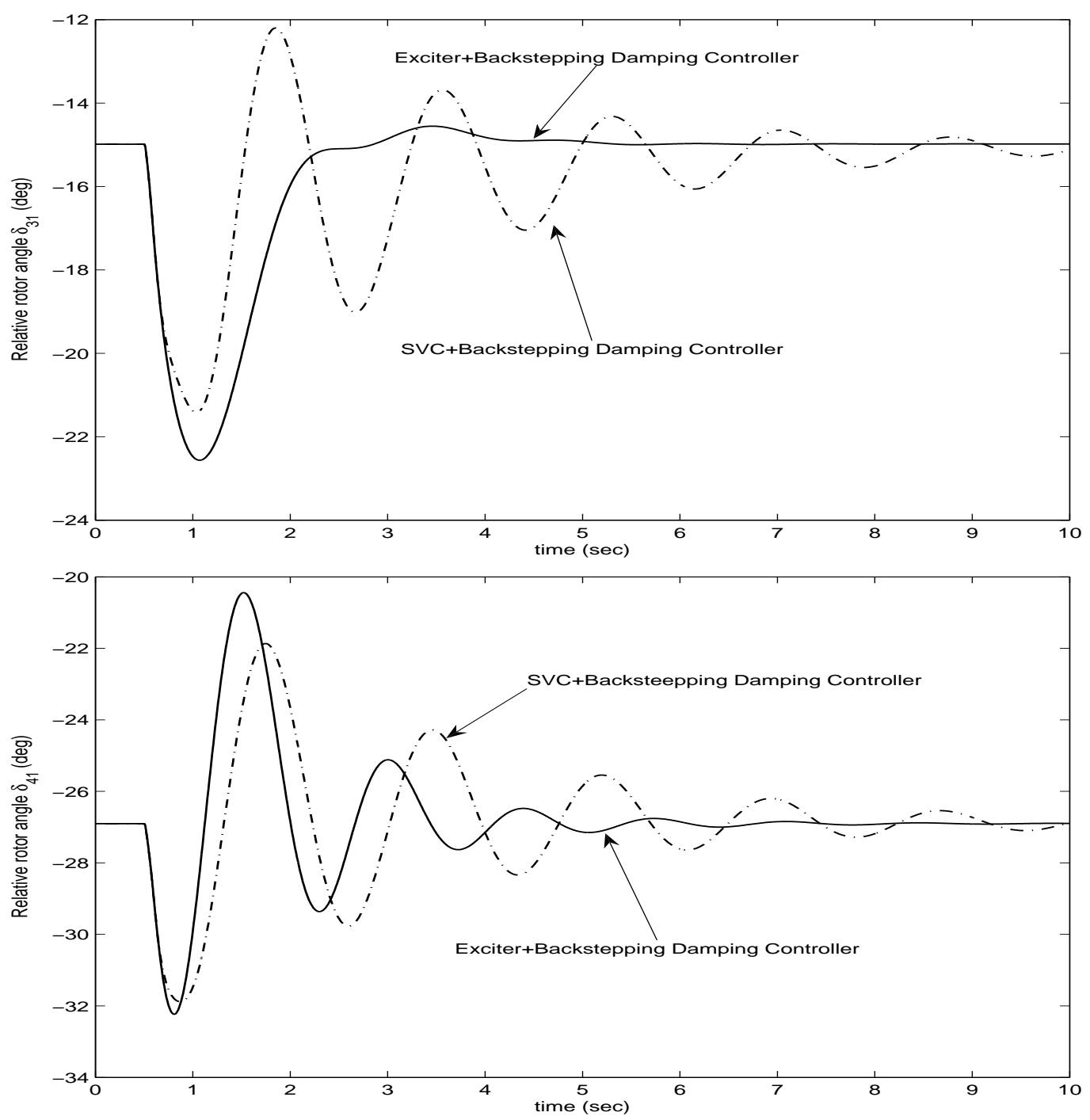

Figure 5.14: Relative rotor angles $\delta_{31}$ and $\delta_{41}$. Solid line (Excitation control with backstepping damping controller), dash-dotted line (SVC with backstepping damping controller) 


\section{Chapter}

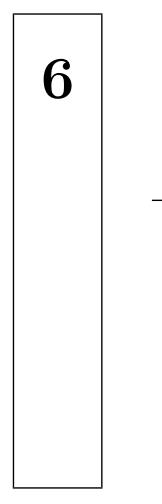

\section{Control Coordination}

\subsection{Optimization and Coordination}

In this section, an optimization based PSO algorithm is proposed to coordinate among multiple nonlinear controllers simultaneously. The coordinated controllers consist of exciter and SVC damping controllers. Exciteation controller is designed based on local measurement at generator bus. SVC damping controller is designed based center of inertia information from each area. Particle swarm has shown a great potential in solving complex optimization problems especially in power system [67]. It has been employed to obtain optimal settings of PSS control parameters in multi-machine power system [2]. In addition it has been used to obtain optimal parameters of SVC damping controllers which is designed based on wide area measurements [22]. One important aspect of this optimization is the criterion used to define the overall goal. Combination of objective functions are considered for simultaneous tuning of controllers that will improve damping in the overall power oscillations in the system in an optimal and globally coordinated manner. 


\subsection{Problem Formulation}

For optimization and coordination of the exciter and SVC need to consider full oscillatory dynamics of the system, especially oscillations on the tie lines where the FACTS devices (i.e. SVC) are installed and oscillations on local generators where the excitation controllers are installed. Therefore the coordinated controllers for excitation and SVC are designed in way to:

- Maximize damping of power oscillations in local and inter-areas

- Enhance overall system stability

Optimization problem is formulated as follow:

$$
\left\{\begin{array}{l}
\underset{\kappa_{s v c}, \kappa_{e x c}}{\operatorname{Min}} \sum_{i=1}^{n} \alpha_{i} J_{i} \\
J_{i}=\int_{0}^{t}\left[\tilde{x}^{T} W \tilde{x}\right] d t \\
\sum \alpha_{i}=1 \\
W=\text { weights } \\
\tilde{x}=\text { state deviation } \\
\kappa_{\text {svc }}=\text { svc control gains } \\
\kappa_{\text {exc }}=\text { exciter control gains }
\end{array}\right.
$$

Regarding damping of power swings, certain state variables need to be considered to form an objective function (6.1). State deviation variables are presented as $\tilde{x}=x-x_{\circ}$. Where the $x_{\circ}$, initial values for states. Weighting matrix $W$ has diagonal elements with dimensionality of $n \times n$ where $n$ stands for the number of variables that are obtained through optimization. The objective of the simultaneous control tuning is to globally optimize the overall system damping performance. This requires simultaneous optimization and coordination of the controllers depends on their placement in the power system. Where FACTS devices are installed on long transmission lines in between areas and excitation system are placed on generators. Therefore, four different objective functions are presented and compared in following. 
- First objective function includes deviation of active power output of generators in areas 1 and 2 plus deviation of active power tie lines which connects each area together. Weighting elements are denoted as $w_{i i}$ and $w_{j j}^{\prime}$. Optimization problem is formulated as follow:

$$
\min J_{1}=\int_{0}^{t}\left[\sum_{i=1}^{4} w_{i i}\left(P_{G e n_{i}}(t)-P_{G e n_{i}}^{\circ}\right)^{2}\right]+\left[\sum_{j=1}^{2} w_{j j}^{\prime}\left(P_{t i e_{j}}(t)-P_{t i e_{j}}^{\circ}\right)^{2}\right]
$$

- Second objective function includes generators' speed as given with respect to synchronous reference frame and voltage deviation at bus 101 where SVC is connected. Optimization of objective function is given as:

$$
\min J_{2}=\int_{0}^{t} \varpi_{1}\left(\Delta \omega_{C O I_{12}}\right)^{2}+\varpi_{2}\left(\Delta V_{\text {bus }}\right)^{2} d t
$$

- Third objective function center of inertia related to electric power (6.10)

$$
\min J_{3}=\int_{0}^{t} \varpi_{3}\left(\Delta P_{C O I_{12}}\right)^{2} d t
$$

- Fourth objective function is a combination of $J_{1}$ and $J_{2}$.

$$
\min _{\alpha_{1}, \alpha_{2}=0.5} J_{\alpha_{1}+\alpha_{2}=1} J_{4}=\alpha_{1} J_{1}+\alpha_{2} J_{2}
$$

Objective function $J_{4}$ is suitable for coordinated design of multiple controllers. This combination provides improvement in damping of overall power oscillations in a globally coordinated manner.

\subsection{Power System Under Study}

Figure 6.1 illustrates the combination of exciter and FACTS controllers for two area system [65]. The system consists of four generators divided into two areas and are connected via two tie lines. Following a disturbance, the two areas swing against each other resulting unstability. In damping control design with backstepping, first the generator model is written in a strict feedback form [64]. In doing so the electric power instead of transient voltages is used as a state variable. Dynamic equations are derived for each generator which is in proper form for control design. In following subsections, damping controller signals for exciter and SVC are briefly explained separately. The approach to obtain excitation damping control design 


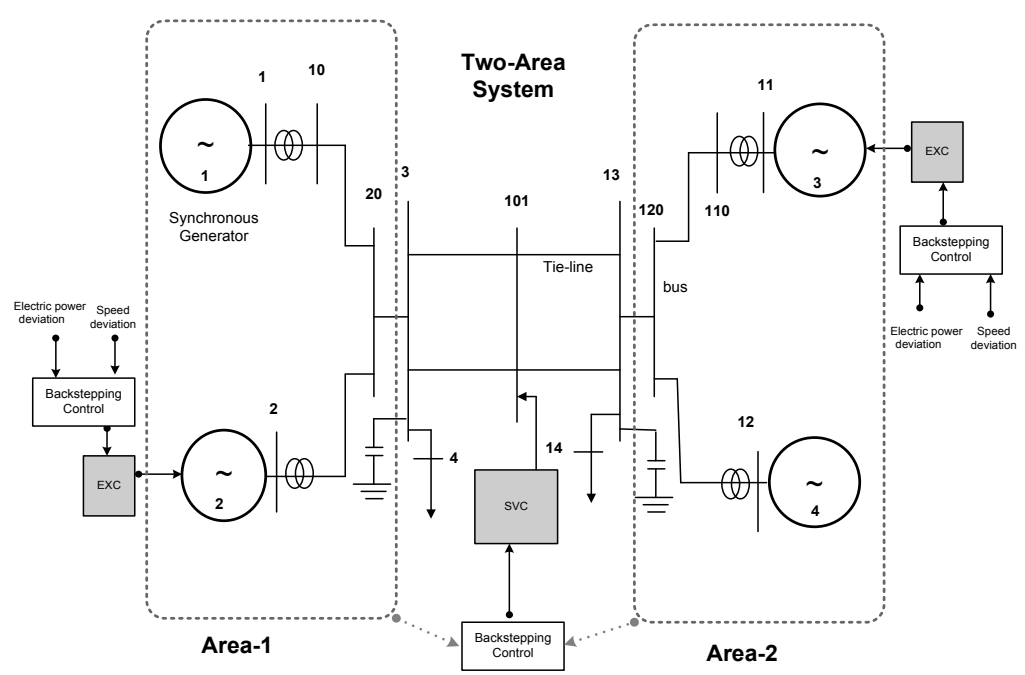

Figure 6.1: Four Machine System with Damping Controllers

is decentralized and based on local generator dynamics with some assumptions about the interface variables to be approximated locally. On the other hand, the approach for SVC damping controller design is centralized and the idea of controller is to drive the area centers of inertia to a stable equilibrium point and keeps the system stable.

\subsubsection{Excitation Control Design}

Damping controller for exciter system is used to modulate the excitation voltage. Dual input signals are feed into controller. Signals are speed and electric power deviations. The control structure is a nonlinear function of angle deviation $\Delta \delta$, speed deviation $\Delta \omega$, and power deviation $\Delta P_{e}$. To obtain the rotor angle deviations, pure integrator from speed signal is used. The output is the stabilizing signal which is added to the excitation system voltage. Based on generic formulation (3.1),(3.2), and (3.5), generator sub-system $\Xi_{i}$ for two axis model is formulated as:

$$
\Xi_{i}\left\{\begin{array}{l}
\Delta \dot{\delta}_{i}=\Delta \omega_{i} \\
\Delta \dot{\omega}_{i}=-\frac{D_{i}}{2 H_{i}} \Delta \omega_{i}-\frac{\omega_{0 i}}{2 H_{i}} \Delta P_{e i} \\
\Delta \dot{P}_{e i}=-\frac{\Delta P_{e i}}{T_{q o i}^{\prime}}+\frac{I_{q i}}{T_{d o i}^{\prime}} \Delta u_{f l d}+d
\end{array}\right.
$$


where parameters are defined as in [55].

$$
\left\{\begin{array}{c}
x_{0 i}=\Delta \delta_{i}, x_{1 i}=\Delta \omega_{i}, x_{2 i}=\Delta P_{e i} \\
f_{0 i}=0, g_{0 i}=1, \\
f_{1 i}=-\frac{D_{i}}{2 H_{i}}, g_{1 i}=-\frac{\omega_{0 i}}{2 H_{i}} \\
f_{2 i}=-\frac{\Delta P_{e i}}{T_{q o i}^{\prime}}, g_{2 i}=\frac{I_{q i}}{T_{d o i}^{\prime}} \\
d_{i}=-\frac{I_{q i} I_{d i}\left(X_{q i}-X_{q i}^{\prime}\right)}{T_{q o i}^{\prime}}+\frac{I_{q i} I_{d i}\left(X_{d i}-X_{d i}^{\prime}\right)}{T_{d o i}^{\prime}}-\frac{P_{e i}^{\circ}}{T_{q o i}^{\prime}} \\
-\frac{u_{f l d}^{\circ} I_{q i}}{T_{d o i}^{\prime}}+E_{q i}^{\prime} I_{q i}\left(\frac{1}{T_{q o i}^{\prime}}-\frac{1}{T_{d o i}^{\prime}}\right)+E_{d i}^{\prime} \dot{I}_{d i}+E_{q i}^{\prime} \dot{I}_{q i}
\end{array}\right.
$$

For designing damping controller for exciter, $\Delta u_{f l d}$ is obtained explicitly through equation (3.30):

- The interconnection term $d_{i}$ between the generators are estimated by $\tilde{d}_{i}$ using local information. The estimated term presents a polynomial of electric power deviations at each generator unit.

$$
\tilde{d}_{i} \approx \theta_{1 i} \Delta P_{e_{i}}+\theta_{2 i} \Delta P_{e_{i}}^{2}
$$

where coefficients $\left(\theta_{1 i}\right.$ and $\theta_{2 i}$ along with $\left.\hat{\beta}_{i}\right)$ are to be estimated through adaption laws $[55]$.

$$
\left\{\begin{array}{c}
\dot{\hat{\theta}}_{1 i}=\Gamma_{1 i}\left(\Delta P_{e i}-\alpha_{1 i}\right) \Delta P_{e i} \\
\dot{\hat{\theta}}_{2 i}=\Gamma_{2 i}\left(\Delta P_{e i}-\alpha_{1 i}\right) \Delta P_{e i}^{2} \\
\dot{\hat{\beta}}_{i}=\gamma_{i}\left(\Delta P_{e i}-\alpha_{1 i}\right) \Delta u_{f l d i}
\end{array}\right.
$$

$\Gamma_{i}, \gamma_{i}$ are weighting factors and required tuning. $\alpha_{1 i}$ is a desired trajectory that is obtained through backstepping control design.

- Nonlinear controller $\Delta u_{f l d i}$ includes gains and weighting factors $\left(\kappa_{1 i}^{e x c}, \kappa_{2 i}^{e x c}, \kappa_{3 i}^{e x c}\right),\left(\Gamma_{1 i}, \Gamma_{2 i}, \gamma_{i}\right)$ that need to be tuned properly .

- Static exciter model is conside for the actuator [78]. Figure 6.2 shows damping controller with exciter. 


\subsubsection{SVC Control Design}

SVC is a FACTS device, which is used primarily for the purpose of voltage and reactive power control [39]. Once installed, a damping control signal can be introduced into voltage summing junction to improve damping. Authors have implemented backstepping control technique for SVC damping controller [56]. The formulation is explained in brief. Consider an Area-i including $n$ number of generators. Center of inertia reference transformation is defined by COI angle, speed and electric power as following:

$$
\delta_{C O I_{i}}=\frac{1}{M_{\sum}} \sum_{j=1}^{n} M_{j} \delta_{j} \quad \omega_{C O I_{i}}=\frac{1}{M_{\sum}} \sum_{j=1}^{n} M_{j} \omega_{j} \quad P_{e C O I_{i}}=\frac{1}{M_{\sum}} \sum_{j=1}^{n} M_{j} P_{e j}
$$

where $M_{\sum}=\sum_{j=1}^{n} M_{j}$ and $M_{i}=\frac{2 H_{i}}{\omega_{0}}$ [81]. Dynamics with center of inertia calculation for area $i$ is simplified as follow. The notation $\sum$ presenting the summations for generator parameters within each area.

$$
\Xi_{C O I_{i}}\left\{\begin{array}{l}
\dot{\delta}_{C O I_{i}}=\omega_{C O I_{i}}-\omega_{0 i} \\
\dot{\omega}_{C O I_{i}}=-\frac{D_{\sum i}\left(\omega_{\sum i}-\omega_{0 i}\right)}{M_{\sum i}}-\frac{\omega_{0 i}\left(P_{e C O I_{i}}-P_{e i}^{\circ}\right)}{M_{\sum i}} \\
\dot{P}_{e C O I_{i}}=-\frac{\left(P_{e C O I_{i}}-P_{e i}^{\circ}\right)}{T_{\sum q o i}^{\prime}}+\frac{I_{\sum q i}^{\prime} q i}{T_{\sum d o i}^{\prime}} \Delta u_{s v c}+d_{\sum i}
\end{array}\right.
$$

Dynamic of relative motion between Area-1 and Area-2 can be formulated from (6.9) where the states are the relative difference between two areas.

$$
\begin{aligned}
\Delta \delta_{C O I_{12}} & =\delta_{C O I_{1}}-\delta_{C O I_{2}} \\
\Delta \omega_{C O I_{12}} & =\omega_{C O I_{1}}-\omega_{C O I_{2}} \\
\Delta P_{C O I_{12}} & =P_{C O I_{1}}-P_{C O I_{2}}
\end{aligned}
$$

Note that

- Dynamic of relative equations is in strict feedback form.

- The generator parameters for two-area system are similar.

- Mean motion between area-1 and area-2 is obtained to design SVC damping control signal $\Delta u_{s v c}$. 
- Damping controller for SVC (3.30) has three gains $\left(\kappa_{1}^{s v c}, \kappa_{2}^{s v c}, \kappa_{3}^{s v c}\right)$ for tuning.

Figure 6.2 shows schematic block diagram for exciter and SVC damping controllers. For SVC, inputs are obtained through wide area measurements and remote information from each area. Remote signals, based on speed and electric power deviations for each area, is fed into damping controller. The output of the controller is added to SVC voltage control loop reference.

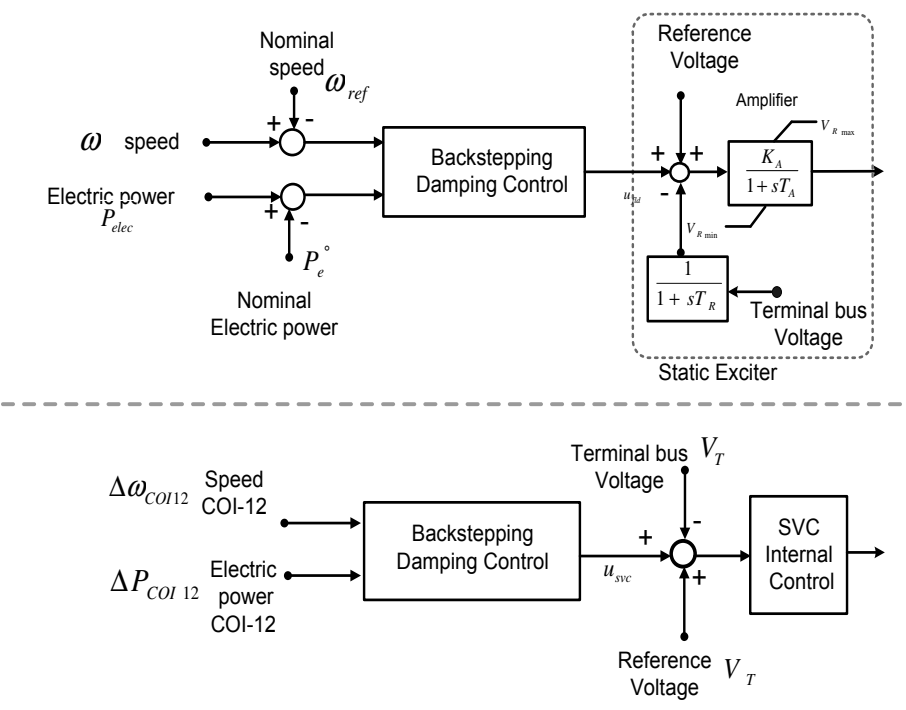

Figure 6.2: Damping Controllers for Exciter and $S V C$

\subsection{Optimum Settings for Controllers}

This section presents a global tuning procedure for nonlinear backstepping damping controllers using Particle Swarm Optimization algorithm [59]. PSO originally is developed for nonlinear optimization problems with continuous variables. In this technique the system is initialized with a population of random solutions. Each potential solution (known as particle) is assigned to a randomized velocity and then move through the problem search space. Velocity and position update equations are given as:

$$
\begin{gathered}
\underbrace{v}_{\text {new velocity }}=K[\underbrace{v}_{\text {velocity }}+c_{1} \text { rand }(\underbrace{\text { pbest }}_{\text {localbest }}-\underbrace{\text { Particle }}_{\text {position }})+c_{2} \text { Rand }(\underbrace{\text { gbest }}_{\text {globalbest }}-\underbrace{\text { Particle }}_{\text {position }})] \\
\underbrace{\hat{\rho}}_{\begin{array}{c}
\text { new } \\
\text { position }
\end{array}}=\underbrace{\hat{\rho}}_{\begin{array}{c}
\text { current } \\
\text { position }
\end{array}}+\underbrace{v}_{\begin{array}{c}
\text { new } \\
\text { velocity }
\end{array}}
\end{gathered}
$$


Where

$$
K=\frac{2}{\left|2-\phi-\sqrt{\phi^{2}-4 \phi}\right|}
$$

and $\phi=c_{1}+c_{2}, \phi>4 . K$ is called constriction factor, $c_{1}$ and $c_{2}$ are weighting factors. For most implementations equal values for both parameters are considered $\left(c_{1}=c_{2}=2.05\right)$. Rand, rand are uniform random numbers [0-1]. When $\phi>4$ the swarm converges toward the best solution in the search space. The main objective of this algorithm is to obtain optimum controller gains and weighting factors simultaneously.

\subsection{Results and Discussion}

Two area four generator system is presented for global optimization and coordination of the exciter and SVC damping controllers (Figure 6.1). The two areas are identical with two parallel tie lines $(230-\mathrm{km})$ which transfer $400 \mathrm{MW}$ from generators 1,2 to generators 3,4 in normal operating condition. After solving the load flow and initialize the system, particle swarm optimization runs to obtain the optimum controller gains for controllers with a defined scenario.

\subsubsection{Case-I}

3-phase fault occurs on line 3-101 for 170 msec. Tables 6.1, 6.2, provide the controller gains with PSO optimization algorithm. Weighting factors for $J_{1}, J_{2}, J_{3}$ are obtained with PSO and shown in Table 6.3. Figure 6.3 shows the comparison between the relative rotor angles for $J_{1}$ (dash line), $J_{2}$ (dash-dot line), $J_{3}$ (dotted line) and $J_{4}$ (solid line). Combination of objective first and second objective functions (presented as $J_{4}$ ) provides better result in damping local and inter-area oscillations in comparison with $J_{1}, J_{2}, J_{3}$. It is clear that with proper choice objective function, the power and controllability of these controllers can be used to increase the damping of oscillation caused by system contingency. Power deviations between tie lines, transmission lines between buses $3-101$ and 101-13, are compared with each other in Figure 6.4. Less power deviations are observed through transferring the bulk of power by optimizing and coordinating the controllers with objective function $J_{4}$. 
Table 6.1: $S V C$ and EXC Controller Gains

\begin{tabular}{c|c|c|c}
\hline \hline $\begin{array}{c}\text { Objective function } J_{1} \\
\text { SVC control gains }\end{array}$ & $k_{1}^{s v c}$ & $k_{2}^{s v c}$ & $k_{3}^{s v c}$ \\
\hline SVC at Bus \#101 & 55 & 15.2 & 25.3 \\
\hline EXC control gains & $k_{1}^{e x c}$ & $k_{2}^{e x c}$ & $k_{3}^{e x c}$ \\
\hline EXC at Generator \#2 & 20 & 80 & 70 \\
EXC at Generator \#3 & 70 & 78 & 20 \\
\hline Optimized EXC $\left(J_{1}\right)$ & $\Gamma_{1}$ & $\Gamma_{2}$ & $\gamma$ \\
\hline EXC at Generator \#2 & 200 & 400 & 51 \\
EXC at Generator \#3 & 700 & 481 & 384 \\
\hline Objective function $J_{2}$ & & & \\
SVC control gains & $k_{1}^{s v c}$ & $k_{2}^{s v c}$ & $k_{3}^{s v c}$ \\
\hline SVC at Bus \#101 & 2.8 & 10 & 1.7 \\
\hline EXC control gains & $k_{1}^{e x c}$ & $k_{2}^{e x c}$ & $k_{3}^{e x c}$ \\
\hline EXC at Generator \#2 & 12.5 & 11.4 & 26.9 \\
EXC at Generator \#3 & 17.6 & 30.9 & 8.8 \\
\hline EXC control gains & $\Gamma_{1}$ & $\Gamma_{2}$ & $\gamma$ \\
\hline EXC at Generator \#2 & 333.5 & 514.8 & 57.2 \\
EXC at Generator \#3 & 750.7 & 553.6 & 102.9 \\
\hline
\end{tabular}


Table 6.2: $S V C$ and EXC Controller Gains

\begin{tabular}{c|c|c|c}
\hline \hline $\begin{array}{c}\text { Objective function } J_{3} \\
\text { SVC control gains }\end{array}$ & $k_{1}^{s v c}$ & $k_{2}^{s v c}$ & $k_{3}^{s v c}$ \\
\hline SVC at Bus \#101 & 9.9 & 1 & 6 \\
\hline EXC control gains & $k_{1}^{e x c}$ & $k_{2}^{e x c}$ & $k_{3}^{e x c}$ \\
\hline EXC at Generator \#2 & 50 & 27.2 & 50 \\
EXC at Generator \#3 & 39 & 42.6 & 10 \\
\hline EXC control gains & $\Gamma_{1}$ & $\Gamma_{2}$ & $\gamma$ \\
\hline EXC at Generator \#2 & 354.3 & 600 & 20 \\
EXC at Generator \#3 & 765.1 & 400 & 200 \\
\hline Objective function $J_{4}$ & & & \\
SVC control gains & $k_{1}^{s v c}$ & $k_{2}^{s v c}$ & $k_{3}^{s v c}$ \\
\hline SVC at Bus \#101 & 4 & 3.5 & 1.4 \\
\hline EXC control gains & $k_{1}^{e x c}$ & $k_{2}^{e x c}$ & $k_{3}^{e x c}$ \\
\hline EXC at Generator \#2 & 47 & 51 & 53 \\
EXC at Generator \#3 & 5.2 & 39 & 18.2 \\
\hline EXC control gains & $\Gamma_{1}$ & $\Gamma_{2}$ & $\gamma$ \\
\hline EXC at Generator \#2 & 529.6 & 415.9 & 50.2 \\
EXC at Generator \#3 & 287.2 & 494.2 & 74 \\
\hline
\end{tabular}



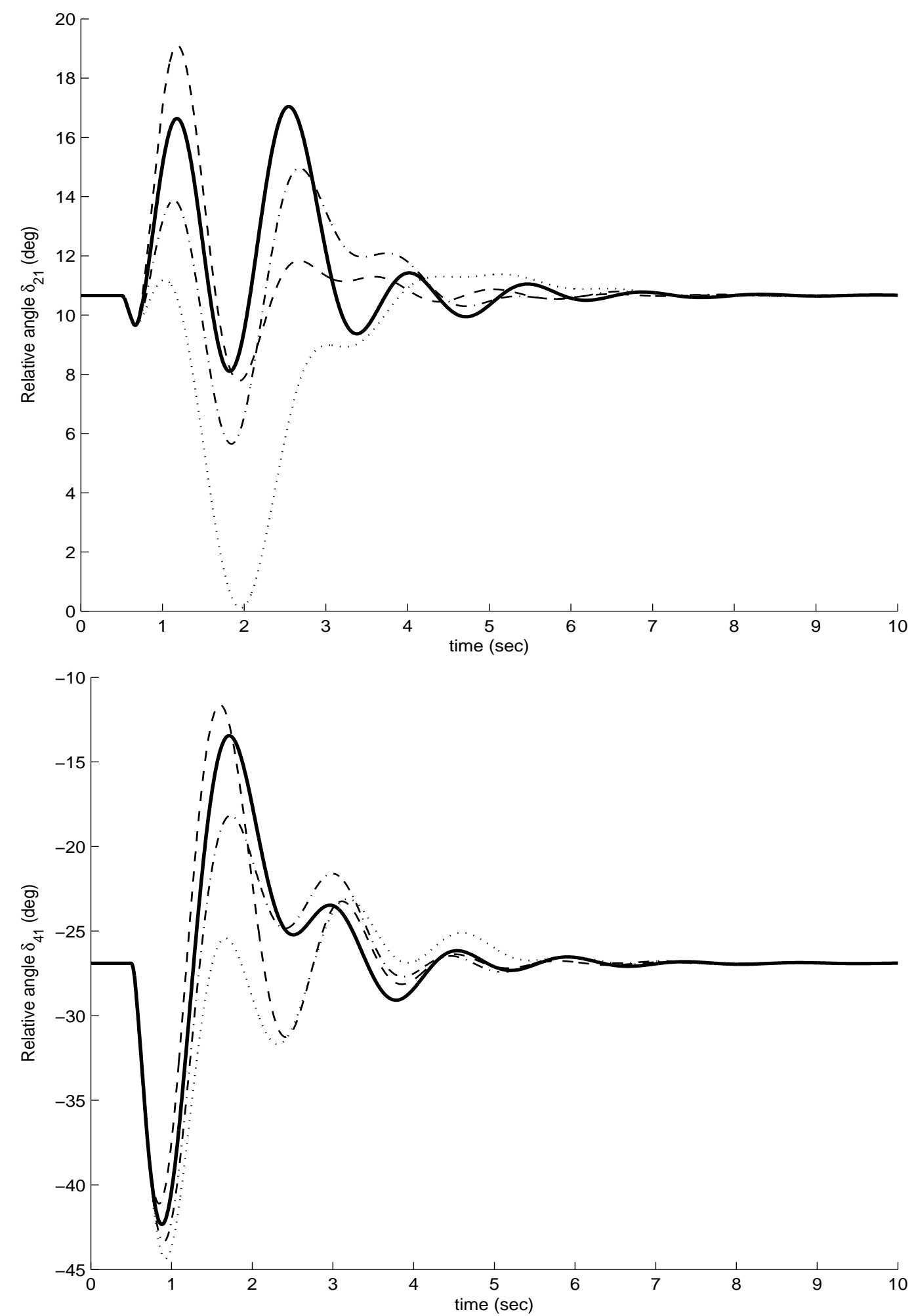

Figure 6.3: Relative rotor angles $\delta_{21}, \delta_{41}$ (deg). Optimized controller gains with $J_{1}$ (dash line), $J_{2}$ (dash-dot line), and $J_{3}$ (dotted line), $J_{4}$ (solid line) 
Table 6.3: Weighting factors for $J_{1}, J_{2}, J_{3}, J_{4}$

\begin{tabular}{c|c|c|c|c}
\hline \hline $\begin{array}{c}\text { Objective Function } \\
\text { weighting factors }\end{array}$ & $J_{1}$ & $J_{2}$ & $J_{3}$ & $J_{4}$ \\
\hline$\varpi_{1}$ & - & 333 & - & 482.8 \\
$\varpi_{2}$ & - & 750 & - & 603.2 \\
$\varpi_{3}$ & - & - & 600 & - \\
\hline$w_{11}^{\prime}$ & 10 & - & - & 611.313 \\
$w_{22}^{\prime}$ & 10 & - & - & 500 \\
\hline$w_{11}$ & 454 & - & - & 99 \\
$w_{22}$ & 99 & - & - & 302.5 \\
$w_{33}$ & 900 & - & - & 312.1 \\
$w_{44}$ & 10 & - & - & 657.8 \\
\hline \hline
\end{tabular}
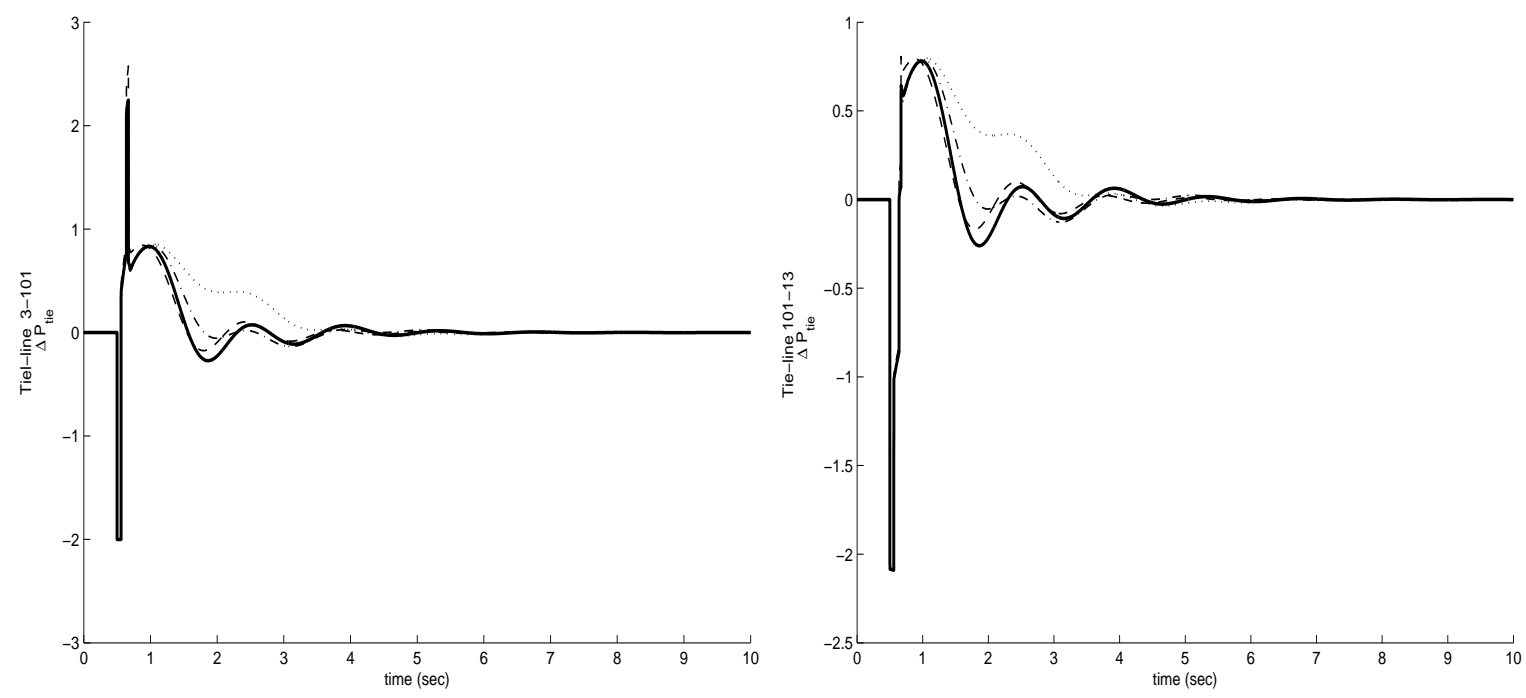

Figure 6.4: Tie-lines power deviations between 3-101 and 101-13. Optimized controller gains with $J_{1}$ (dash line), $J_{2}$ (dash-dot line), $J_{3}$ (dotted line), $J_{4}$ (solid line) 


\subsubsection{Case-II}

Objective function $J_{1}$ in equation (6.2) provides active power deviation of generators and tie line deviation from their nominal values. Their nominal values are obtained through load flow solution. Two different percentage of power generation settings for generators are considered $35 \%$ and $25 \%$ from their nominal values of generation. Then based on the new initialized settings, the optimization is performed to obtain optimum sets of controller gains. Table 6.4 summarizes the results which were obtained through system response with predefined 3-phase fault on line 3-101 for $170 \mathrm{msec}$. Nominal values of gains are provided in Table 6.1 where nominal total active power generation and load are $P_{\sum \text { gen }}=28.27$ (p.u.) $P_{\sum \text { load }}=27.38$ (p.u.) respectively with base of 100 MVA. Figures 6.5 and 6.6 show the advantage of re-tuning for controller gains for different generation settings in comparison with

Table 6.4: Exciter and SVC Controller Gains-25\% and 35\% of active power generation and load

\begin{tabular}{c|c|c|c}
\hline $\begin{array}{c}25 \% \text { change of generation } \\
\text { SVC control gains }\end{array}$ & $k_{1}^{\text {svc }}$ & $k_{2}^{\text {svc }}$ & $k_{3}^{\text {svc }}$ \\
\hline SVC at Bus \#101 & 19.4 & 4.3 & 3.4 \\
\hline EXC control gains & $k_{1}^{\text {exc }}$ & $k_{2}^{\text {exc }}$ & $k_{3}^{\text {exc }}$ \\
\hline EXC at Generator \#2 & 39.7 & 44.1 & 80 \\
EXC at Generator \#3 & 75 & 44.9 & 0.1 \\
\hline EXC control gains & $\Gamma_{1}$ & $\Gamma_{2}$ & $\gamma$ \\
\hline EXC at Generator \#2 & 550 & 884.7 & 48.2 \\
EXC at Generator \#3 & 400 & 700 & 412.5 \\
\hline $35 \%$ change of generation & & & \\
SVC control gains & $k_{1}^{s v c}$ & $k_{2}^{s v c}$ & $k_{3}^{s v c}$ \\
\hline SVC at Bus \#101 & 1.02 & 8.0 & 4.5 \\
\hline EXC control gains & $k_{1}^{e x c}$ & $k_{2}^{e x c}$ & $k_{3}^{e x c}$ \\
\hline EXC at Generator \#2 & 50 & 16 & 48 \\
EXC at Generator \#3 & 50 & 28.1 & 0.01 \\
\hline EXC control gains & $\Gamma_{1}$ & $\Gamma_{2}$ & $\gamma$ \\
\hline EXC at Generator \#2 & 450 & 878.5 & 37.1 \\
EXC at Generator \#3 & 600 & 553.8 & 60 \\
\hline
\end{tabular}



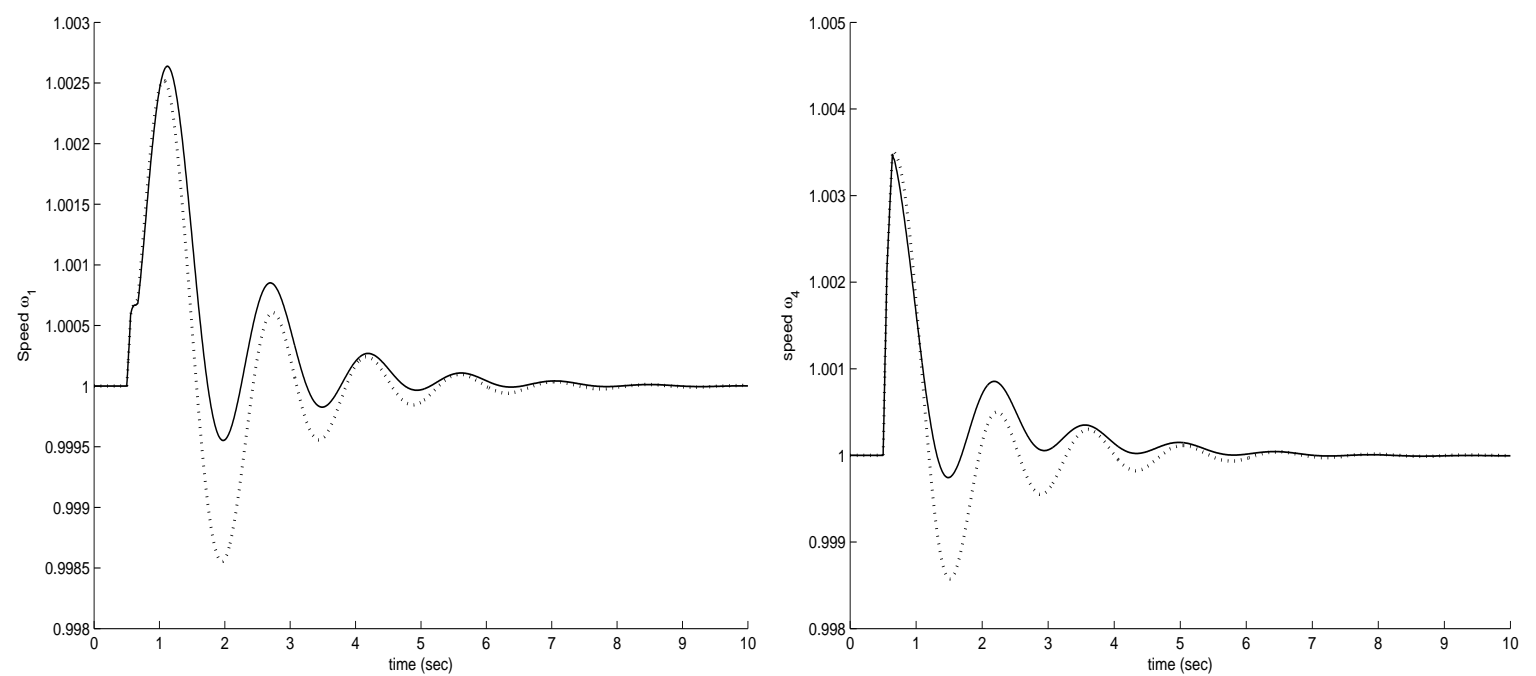

Figure 6.5: Speed deviations Generators 1,4. Performance of controller with nominal gains (dotted line), effect of re-tuned control gains at $35 \%$ change of power generation $P_{\text {gen }}$ (solid line)
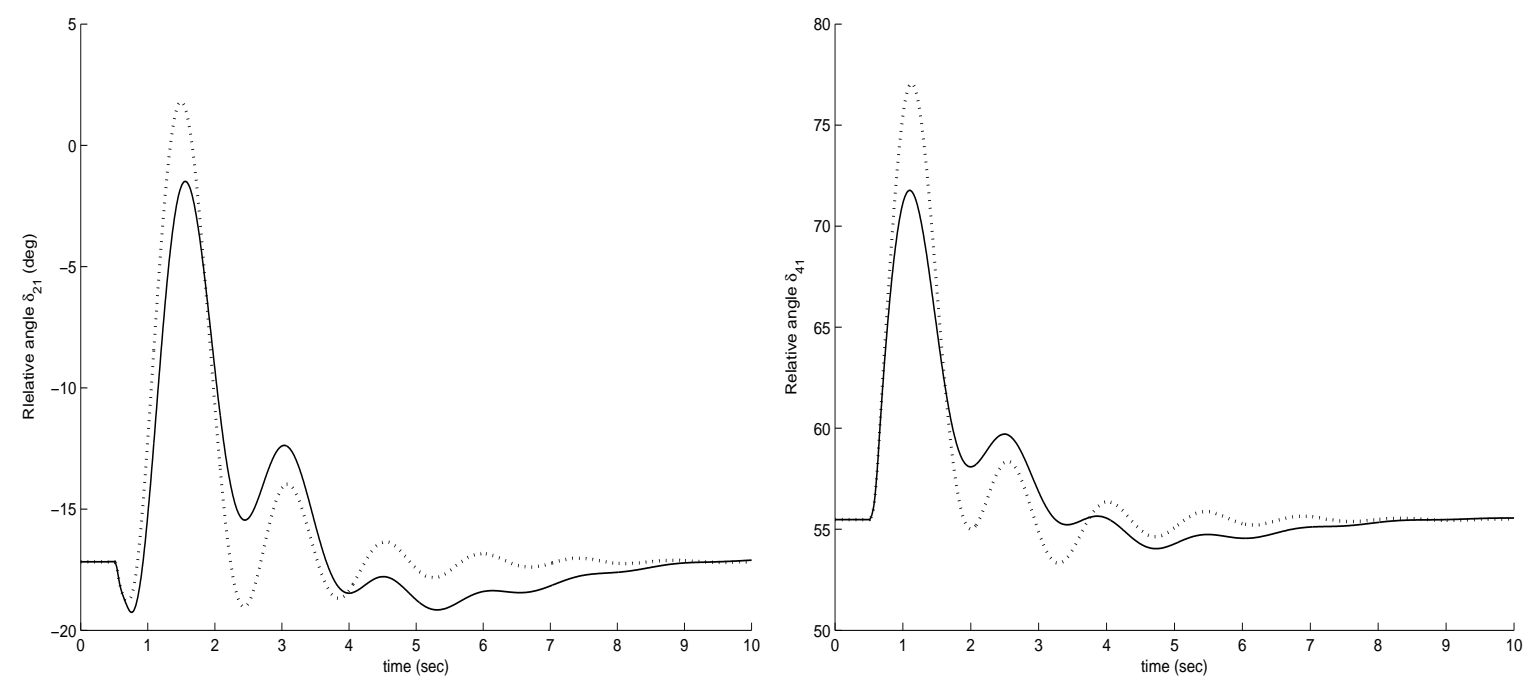

Figure 6.6: Relative angle of generators $\delta_{21}, \delta_{41}$. Performance of controller with nominal gains (dotted line), effect of re-tuned control gains at $35 \%$ change of power generation $P_{\text {gen }}$ (solid line)

gains in nominal condition. Solid line shows the response once the gains are re-tuned and dotted line shows the response with their nominal gains.

Deviation of tie line power oscillations are compared and shown in Figures 6.7. As a result the re-adjustment of controllers gains provide more damping to the system oscillations. Similar conclusion can be derived with different percentage of power generation and load,25\% change. Following results (Figures 6.8, Figure 6.9) show the relative angles and speed of 

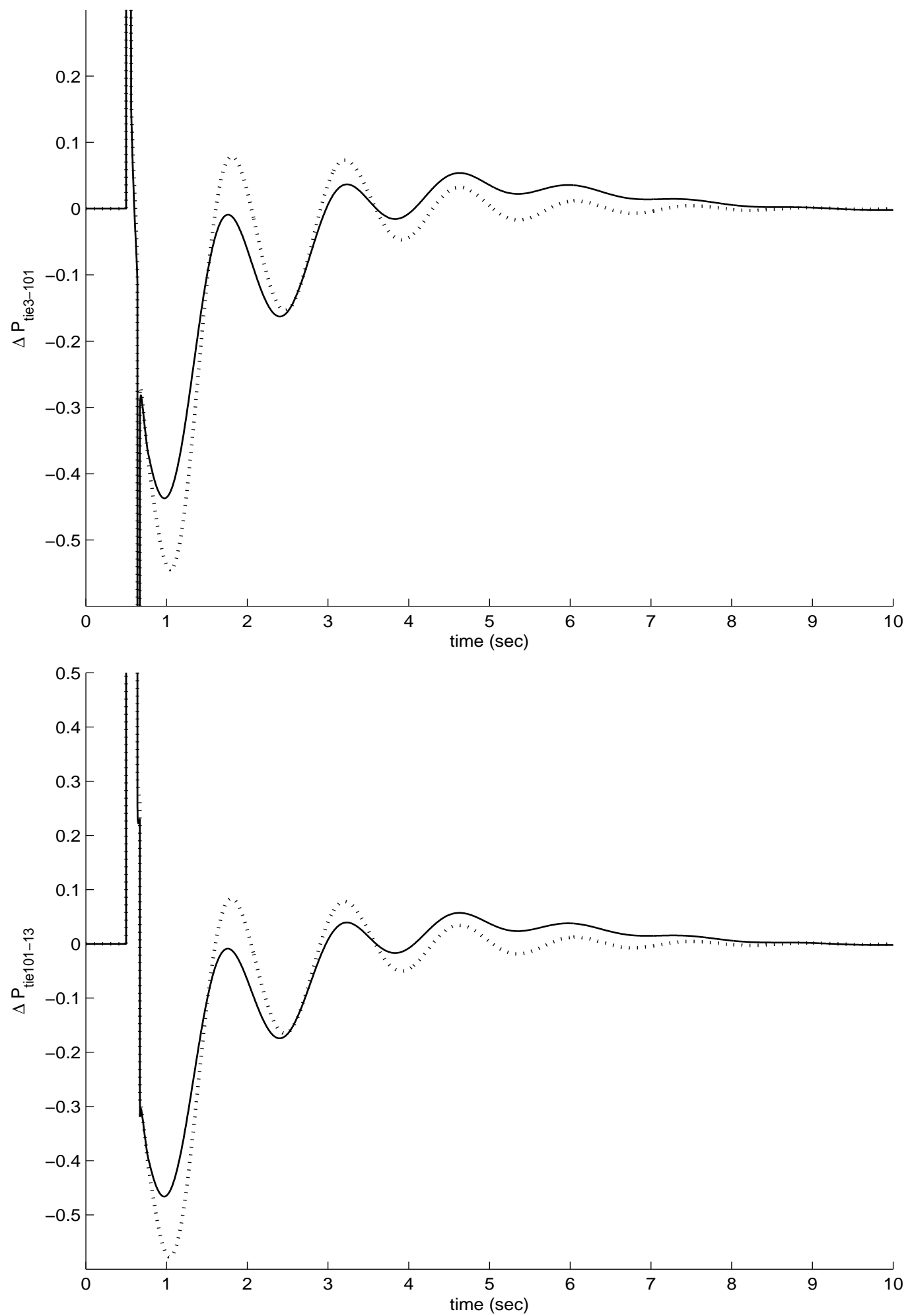

Figure 6.7: Tie line 3-101 power deviations. Performance of controller with nominal gains (dotted line), effect of re-tuned control gains at $35 \%$ change of power generation $P_{\text {gen }}$ (solid line) 

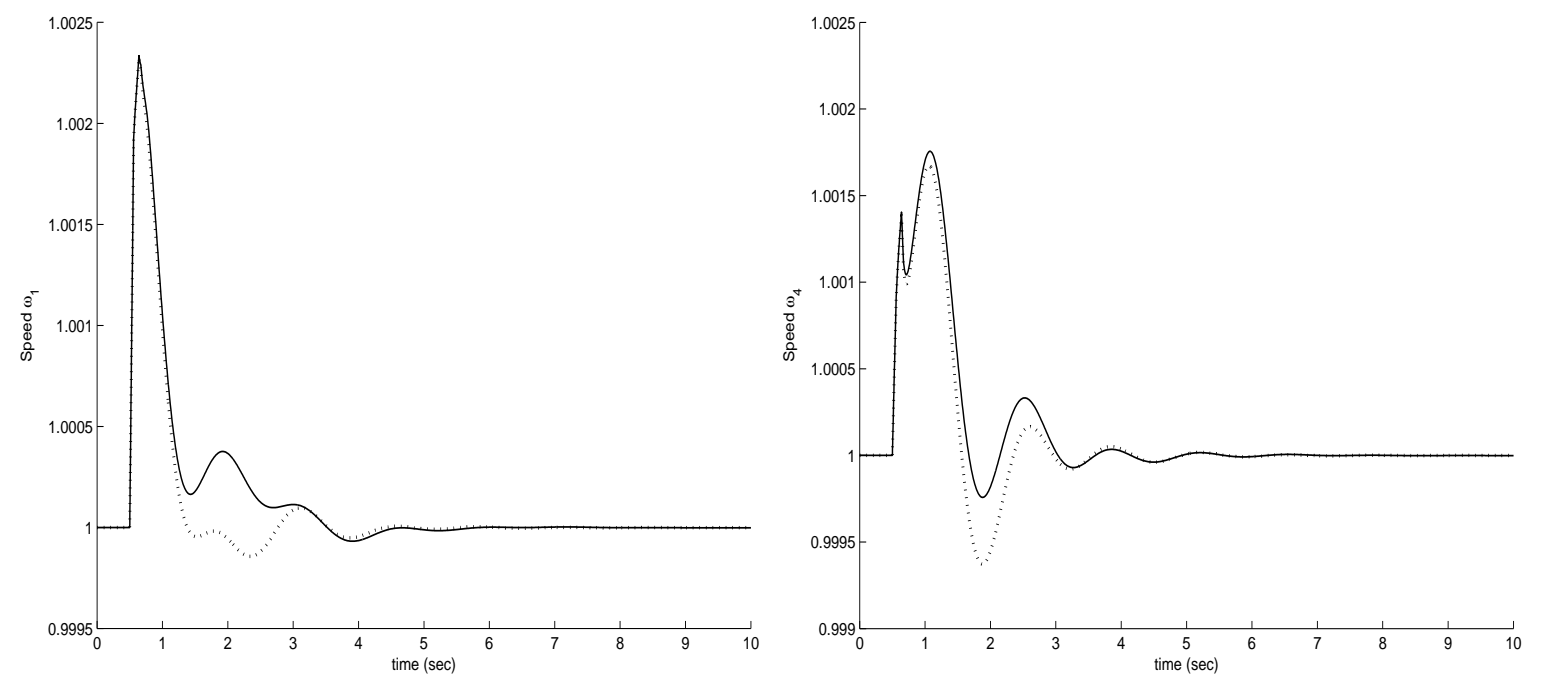

Figure 6.8: Speed deviations Generators 1,4. Performance of controller with nominal gains (dotted line), effect of re-tuned control gains at $25 \%$ change of power generation $P_{\text {gen }}$ (solid line)
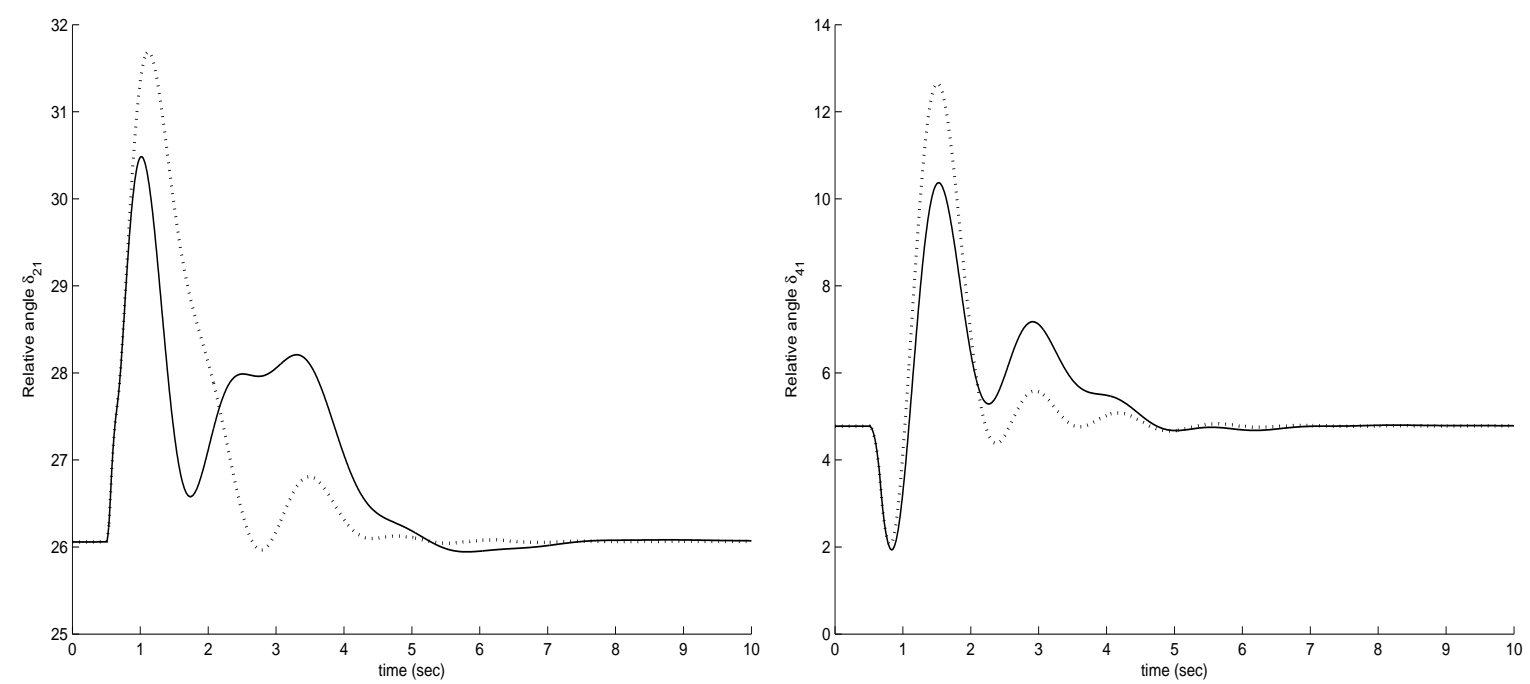

Figure 6.9: Relative angle of generators $\delta_{21}, \delta_{41}$. Performance of controller with nominal gains (dotted line), effect of re-tuned control gains at $25 \%$ change of power generation $P_{\text {gen }}$ (solid line)

generators. In summary, nonlinear coordinated excitation and SVC controllers are proposed to enhance the stability of multi-machine power system. The proposed coordinated controller is designed based on backstepping technique and consists of generator excitation and SVC damping controllers. The goal is to allow the controllers work simultaneously and improve the power system performance cooperatively. The problem is formulated as an optimization problem based on PSO so as to obtain the optimum control gains, and as a result enhance 
power system damping. Simulation results show that by selecting proper objective function

during optimization of nonlinear controllers, notable improvement on system performance and optimization are obtained. 


\subsection{Advanced Control Technique}

In power systems, control devices are installed to enhance various aspects of system stability such as transient stability. These equipments are mainly power electronic based. Given the nature of the power system, it is best suited for the control design where local controllers are coordinated to obtain the stability of overall system. A supplementary damping controller is added to the primary regulator of transmission device or to generator controls. Specifically, this section presents usage of excitation controls of the generators to enhance the stability of system in combination with Reinforcement Learning (RL) algorithm [85].

The combination of RL and backstepping control techniques is proposed to damp oscillations in power systems thru the excitation system. A schematic of the proposed controller for a simple two controllers is shown in Figure 6.10. Backstepping controllers alone are shown in (Figure 1.A). To obtain the control gains, each subsystem is modeled as an independent uncertain dynamic subsystem, where the uncertainty is a disturbance that represents the effects of the rest of the system on that particular subsystem. This disturbance is expressed as a polynomial function of local states, and its parameters are adapted using a Particle Swarm Optimization method. The proposed controller shown in Figure 1.B uses RL at a higher level to adapt the gains due to varying operating conditions and uncertainties in the interface variables. Explanation of the algorithm is as follows.

\subsubsection{Reinforcement Learning}

Reinforcement learning is learning to take actions by observing the current state of the system in order to maximize the long term reward [85]. The controller will discover which action should be taken by interacting with its environment and trying different actions which may lead to the highest reward. Schematic block diagram related to reinforcement learning is shown in Figure 6.11. The controller interacts with the environment and takes an action $a$ from a set of actions $\{A\}$ at time $t$. These actions will affect the environment and takes it to new state $s_{t+1}$. The controller is rewarded for this action, and obtains the reward $r_{t+1}$. The interaction between controller and the environment is repeated until the desired goal is obtained. Conceptually each RL problem has following important components:

- State: Series of information from the system that determines the degree of closeness to 


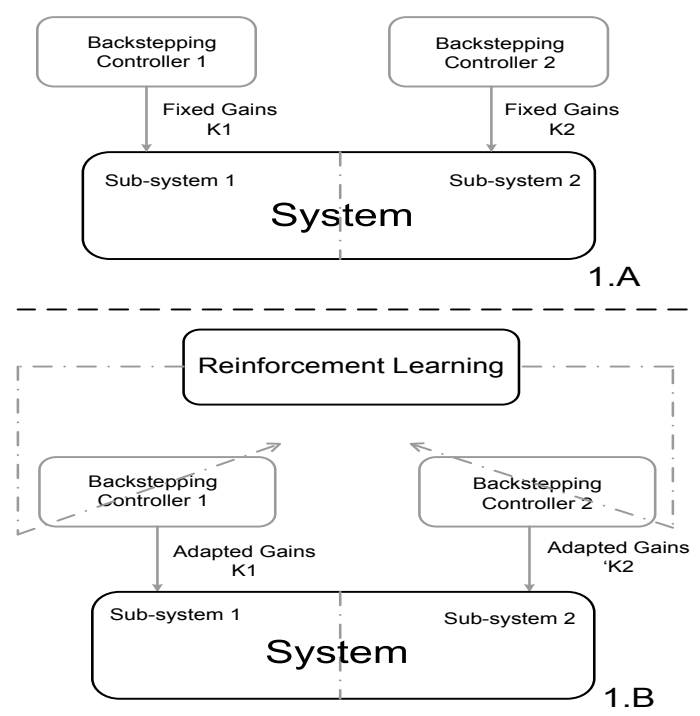

Figure 6.10: Schematic Design of Controlled System

the objective

- Action: Decision made by controller that will affect the environment or system under control

- Reward: Scalar signal that determines how close the action is to the objective

- Policy: Matching between states and actions

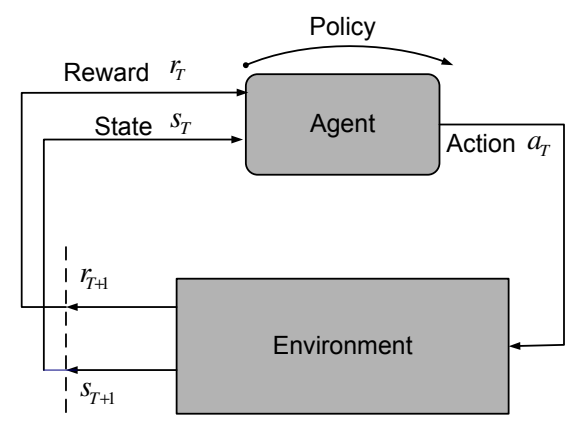

Figure 6.11: Block diagram representation of reinforcement learning

The reward function plays an important role in determining the interaction between agent and environment. The better the definition of the reward function is, the better control 
performance will be. The role of RL methods is to find the policy resulting in the maximum long term reward. The sum of the expected reward in the future is defined as a return of a system and defined as:

$$
R_{t}=\sum_{k=0}^{\infty} r_{t+k+1}
$$

In many applications a discounted factor $0 \leq \gamma \leq 1$ is introduced and the return is modified so that the control will maximize a discount return defined by:

$$
R_{t}=\sum_{k=0}^{\infty} \gamma^{k} r_{t+k+1}
$$

The discount factor is included in the equation to determine the current value of the future reward (6.13). If the objective is to just maximize the immediate reward achieved by taking action $a_{t}$ then $\gamma=0$. When $\gamma=1$ then the equation become the classical definition of the returns. Each RL include two main important functions:

- Value Function: The value of each state $s$ called the state value function, is defined as the expected return when starting at state $s_{t}$ using policy $\Pi(s, a)$. It is given by (6.15):

$$
V_{s}^{\pi}=E_{\pi}\left\{\sum_{k=0}^{\infty} \gamma^{k} r_{t+k+1} \mid s_{t}=s\right\}
$$

- Action Value Function: The action value function of each state $s$ and action $a$, is defined as the expected return, or expected discount reward, when starting at state $s_{t}$, taking action $a_{t}$, using policy $\Pi(s, a)$. It is known as Q-function and it is given in following:

$$
Q^{\pi}(s, a)=E_{\pi}\left\{\sum_{k=0}^{\infty} \gamma^{k} r_{t+k+1} \mid s_{t}=s, a_{t}=a\right\}
$$

The reinforcement learning task is to find the optimal policy that maximizes the value function for all the states. The optimal policy will also maximize the optimal action value function for all the states and actions

$$
Q^{*}\left(s^{*}, a^{*}\right)=\max _{\pi} Q^{\pi}\left(s^{*}, a^{*}\right)
$$

The optimality conditions for these functions are given by the Bellman equation. Optimal policy can be obtained by solving a Bellman equation, assuming that transition probabilities and 
rewards are known. However, in most cases these parameters are not available to controller. Therefore methods which are less dependent on system model are required. Non-model based methods first approximate the system and estimate the Q-function before solving Bellman's equation.

Q-learning is one of the most important Temporal Difference (TD) algorithms which is model independent. In this method the experience is gained by interacting with the environment to update the value function at each time step. As a result the optimal Q-function is approximated directly, without finding the optimal policy. The following equation is used to update the action value functions at each time step.

$$
\Delta Q=\alpha\left[r_{t+1}+\gamma \max _{a} Q\left(s_{t+1}, a_{t+1}\right)-Q\left(s_{t}, a_{t}\right)\right]
$$

In this equation, $\alpha$ is a constant step-size parameter $(0<\alpha<1)$, which represents the amount of the corrected error and $\gamma$ is the discounted factor. The following algorithm summarizes the steps of Q-learning algorithm.

- Initialize $Q(s, a)$ for all the states and the actions

- Repeat for each run of the algorithm

Initialize $s$

Repeat for each step

Take action $a$ based on policy determined by $\mathrm{Q}^{1}$

Observe $s_{t+1}$ and $r$

Modify $Q$ based on equation (6.18)

$s=s_{t+1}$

- Repeat until the terminal state is reached.

\subsection{Proposed Control Design}

Combination of the backstepping controller with reinforcement learning provides optimal performance of the closed loop system. The update of the controller gains is based on reinforcement learning states. State of reinforcement learning is a derivative of Lyapunov function.

\footnotetext{
${ }^{1}$ In order to select an action the $\epsilon$-greedy policy is used. This approach selects the action with the currently highest action value as experienced through interaction with the environment with the probability of $(1-\epsilon)$ and a random action with probability $\epsilon$.
} 


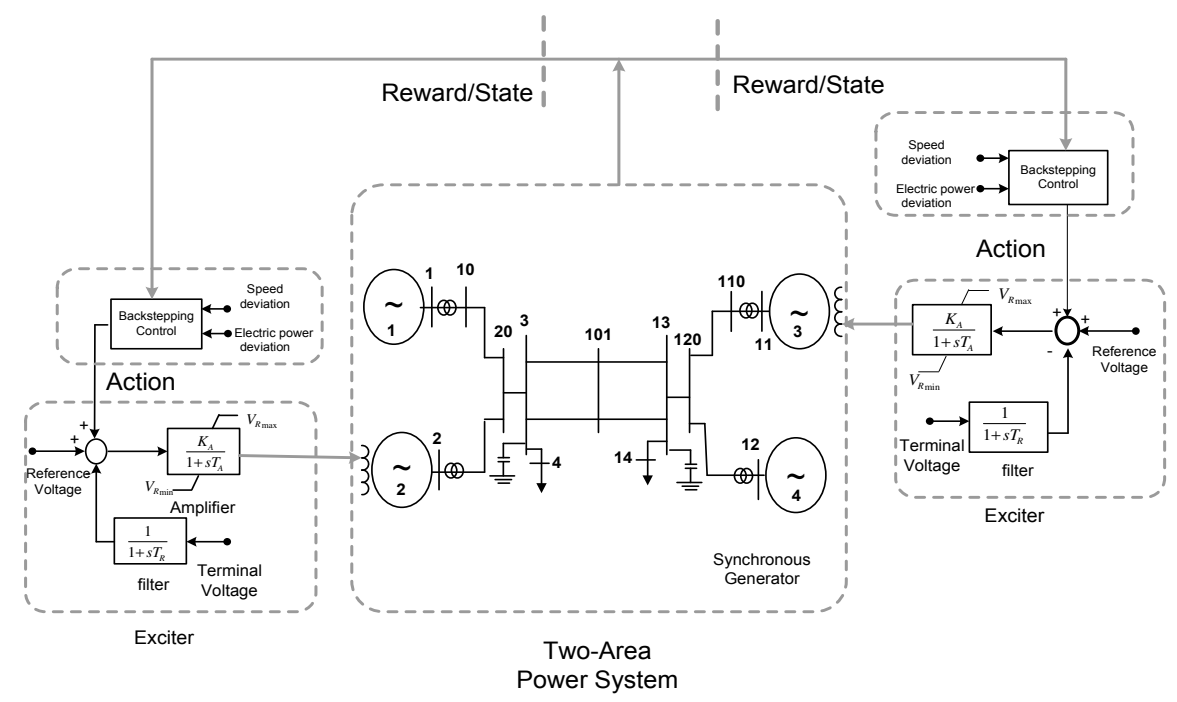

Figure 6.12: Schematic view of backstepping control architecture with reinforcement learner for two area power system

The controller itself contains the backstepping damping controller plus the exciter. Action is a control signal that modifies the exciter field voltage. The environment is a power system. The state of reinforcement learning $(J)$ is presented as:

$$
J=\sum_{i=1}^{m} \underbrace{\dot{V}_{i}}_{\text {uncontrolled Gen }}+\sum_{j=1}^{n} \underbrace{\dot{V}_{j}}_{\text {controlled Gen }}+\sum_{q=1}^{l} \underbrace{\Phi\left(\Delta P_{\text {tie }}\right)}_{\text {coupling }}
$$

Schematic view of for two area system is shown in Figure 6.12. Information from the system that determines the degree of closeness to the objective is obtained through $J$. For controlled generators derivative of Lyapunov function is obtained through backstepping design and similar to (4.65). Similarly, for uncontrolled generators the same transformation can be applied to obtain the local Lyapunov functions.

$$
\dot{V}_{i}=-\left(\Delta \delta-\alpha_{0}\right)^{2}-\left(\Delta \omega-\alpha_{1}\right)^{2}-\left(\Delta P_{e}-\alpha_{2}\right)^{2}
$$

The $\Phi\left(\Delta P_{\text {tie }}\right)$ is a function of tie line power deviation (refer to Appendix $\mathrm{G}$ ).

The initial value for gain settings has important effect on transient behavior of dynamic system especially at the beginning of the interaction with no experience. Optimization search 
technique based on PSO has been proposed by Kennedy et al. [59] and is used off-line to obtain initial settings. These values let the RL driven control interact with the simulation model of the system. Once the initial backstepping controller gains are sufficiently tuned offline, one may implement the proposed technique on real system where it will benefit from the tuned gains obtained in the simulation environment and still be able to improve its behavior from interaction with the environment.

Particle swarm is a well defined methodology in evolutionary computation [59]. Complete survey and implementation including applications in power system is given by Lee et al. [67]. This method requires only primitive mathematical operators and computation requires small amount of memory. This technique is developed through the simulation of bird flocking. Each individual (particle) has a position and a velocity. The information can be updated by changing the velocity and position of particles. The particle itself is a candidate solution in search space.

The main idea is to evaluate the objective function for each particle vector. Each particle vector knows its best values so far, which is denoted by pbest. Also each particle vector knows the best value among all individual best, known as global best or gbest. Then it modifies its movement in the search space by using the following information:

- current position

- current velocity

- distance between current position and pbest

- distance between current position and gbest.

Velocity and position update equations are given as:

$$
\begin{gathered}
\underbrace{v}_{\text {new velocity }}=K\left[\begin{array}{l}
\underbrace{v}_{\text {velocity }}+c_{1} \text { rand }(\underbrace{\text { pbest }}_{\text {localbest }}-\underbrace{\text { Particle }}_{\text {position }}) \\
+c_{2} \text { Rand }(\underbrace{\text { gbest }}_{\text {globalbest }}-\underbrace{\text { Particle }}_{\text {position }})
\end{array}\right] \\
\underbrace{\hat{\kappa}}_{\substack{\text { new } \\
\text { position }}}=\underbrace{\hat{\kappa}}_{\substack{\text { current } \\
\text { position }}}+\underbrace{v}_{\substack{\text { new } \\
\text { velocity }}}
\end{gathered}
$$


Where

$$
K=\frac{2}{\left|2-\phi-\sqrt{\phi^{2}-4 \phi}\right|}
$$

and $\phi=c_{1}+c_{2}, \phi>4$. $K$ is called constriction factor, $c_{1}$ and $c_{2}$ are weighting factors, and $\kappa$ presents the particles positions. In this study particles are presented as controller gains. For most implementations equal values for both parameters are considered $\left(c_{1}=c_{2}=2.05\right)$. Rand, rand are uniform random numbers $[0-1]$. When $\phi>4$ the swarm converges toward the best solution in the search space. This constriction method results in particle convergence over time [59].

\subsection{Case Study}

To illustrate capabilities of the proposed control technique on excitation control, the fourmachine system model [65] is used. The one line diagram of the test system is given in Figure 6.12. All generators are equipped with static exciter (Table 6.5). Generators 2, 3 have

Table 6.5: Parameters for Static Excitation

\begin{tabular}{c|c}
\hline Exciter & $1,2,3,4$ \\
\hline$K_{A}$ Regulator gain p.u. & 200 \\
\hline$T_{A}$ Regulator time constant p.u. & 0.05 \\
\hline$T_{r}$ Filter time constant p.u. & 0.01 \\
\hline$V_{\text {Rmax }}$ upper bound for saturation p.u. & 10 \\
\hline$V_{\text {Rmin }}$ lower bound for saturation p.u. & -10 \\
\hline
\end{tabular}

supplementary controllers. Two area system configurations, with $400 \mathrm{MW}$ of power flowing from area 1 to area 2, were analyzed

- Operating Condition 1- System with two transmission lines between bus 13 and 101

- Operating Condition 2- System with a single line between bus 13 and 101

By analyzing the stability of these two conditions, the ability of the proposed controller to withstand a loss of a line after three phase fault at bus 3 is evaluated. The results are 

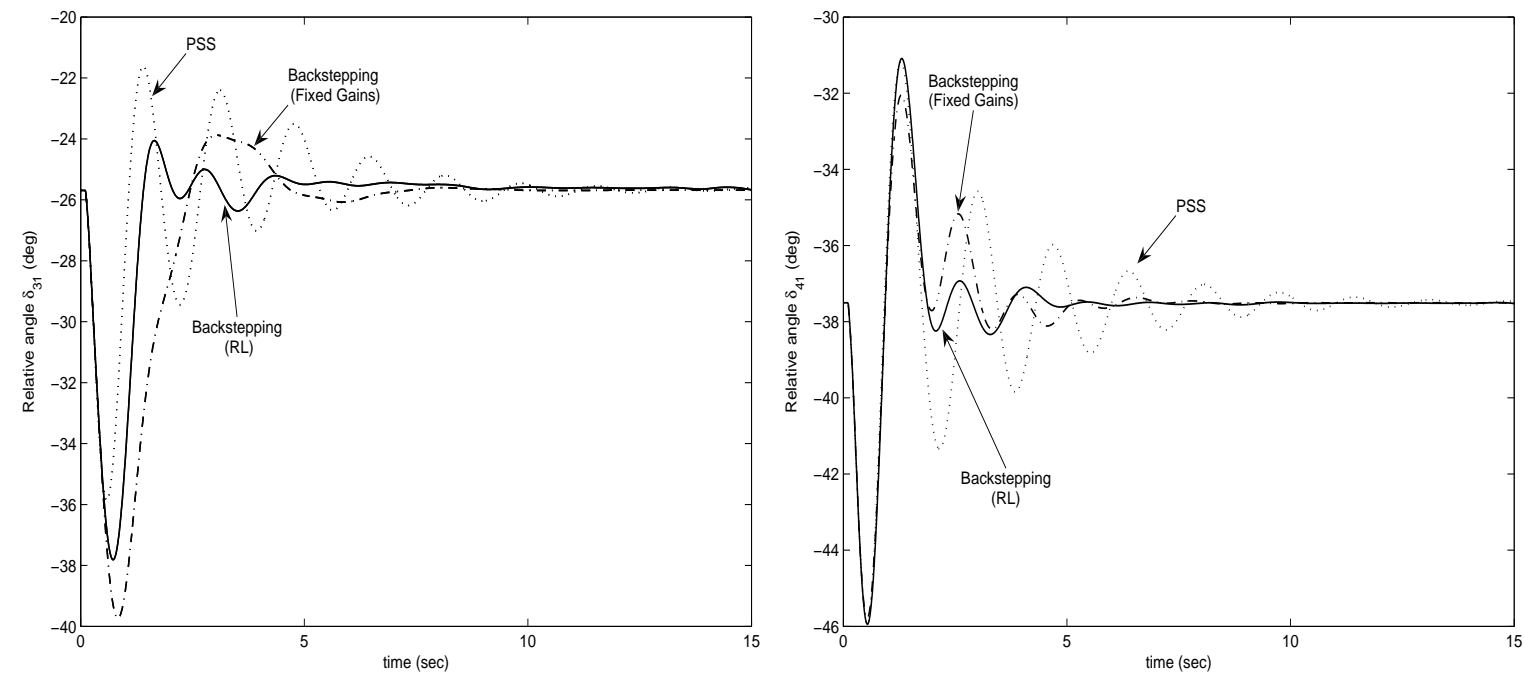

Figure 6.13: Scenario-1 Relative rotor angles $\delta_{31}, \delta_{41}$. Proposed control (solid line), Backstepping with fixed gains (dash-dotted line), PSS (dotted line)

compared with fixed gain backstepping controller tuned by PSO and fixed gain linear PSS tuned by genetic algorithm [37].

The fixed gain backstepping controller is tuned first based on PSO technique [55] to yield the best damping performance characteristic to the system for the particular contingency. Then RL is used to re-adjust the gains under large disturbances at different operating points.

The first scenario is three phase fault at time $t=0.1 \mathrm{sec}$, while the power $(400 \mathrm{MW})$ is flowing from area 1 to area 2. The near end of the line is opened and the line is removed at $t=0.19 \mathrm{sec}$. The line is reconnected back at $0.21 \mathrm{sec}$. Figures 6.13 show the relative angles $\delta_{31}, \delta_{41}$ respectively. Results show the superiority of the proposed reinforcement learning based backstepping control over the fixed gain controller. Adjustment of controller gains $k_{1 i}^{\prime}, k_{2 i}^{\prime}, k_{3 i}^{\prime}$ for generators $i=2,3$ are shown in Figures 6.14.

For the second case with one transmission line between bus $13-101$, the contingency occurs at the line $3-101$ at 1.00 second. The line is removed and fault is cleared at 1.06 sec. The transmission line $3-101$ is reconnected at 2.5 sec. Figures 6.16 show the comparison between relative angles. Fixed gain PSS becomes unstable due to long fault duration and highly stressed transmission lines. Backstepping controller provides desired response with readjusted gains (Figure 6.17). Terminal voltages for generators 2 are shown in Figures 6.18. 

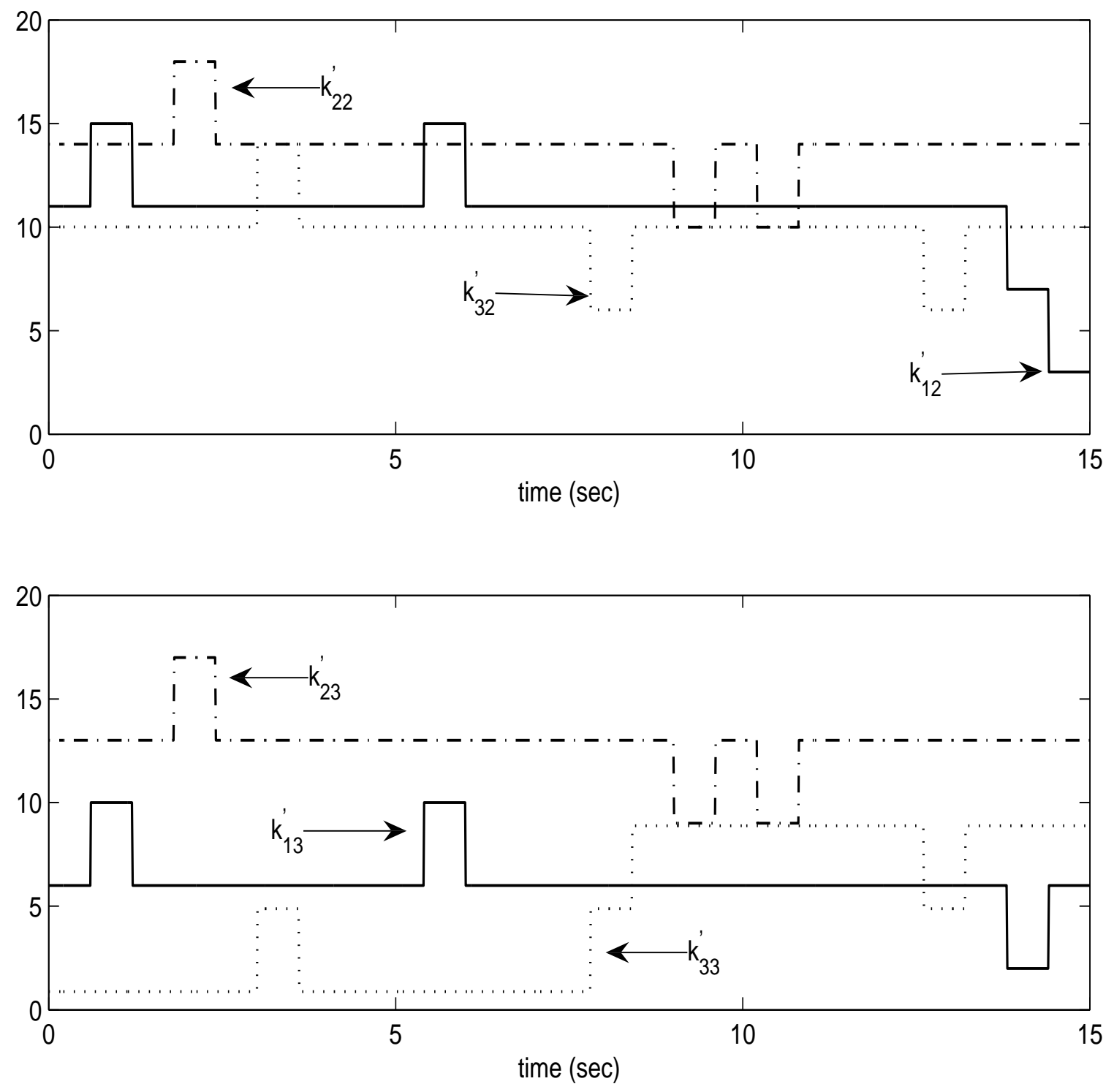

Figure 6.14: Scenario-1 Control gains for generator 2,3 

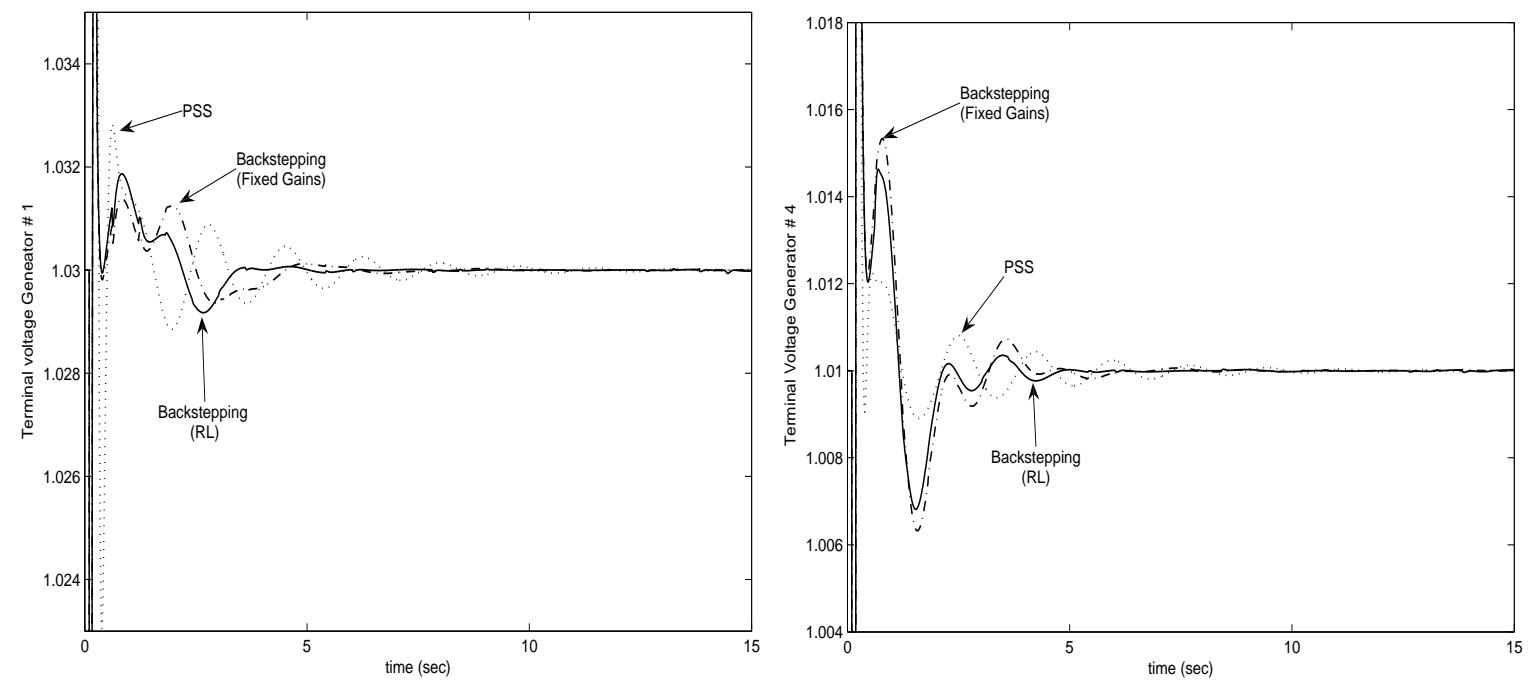

Figure 6.15: Scenario-1 Terminal voltages for Generators 1, 4
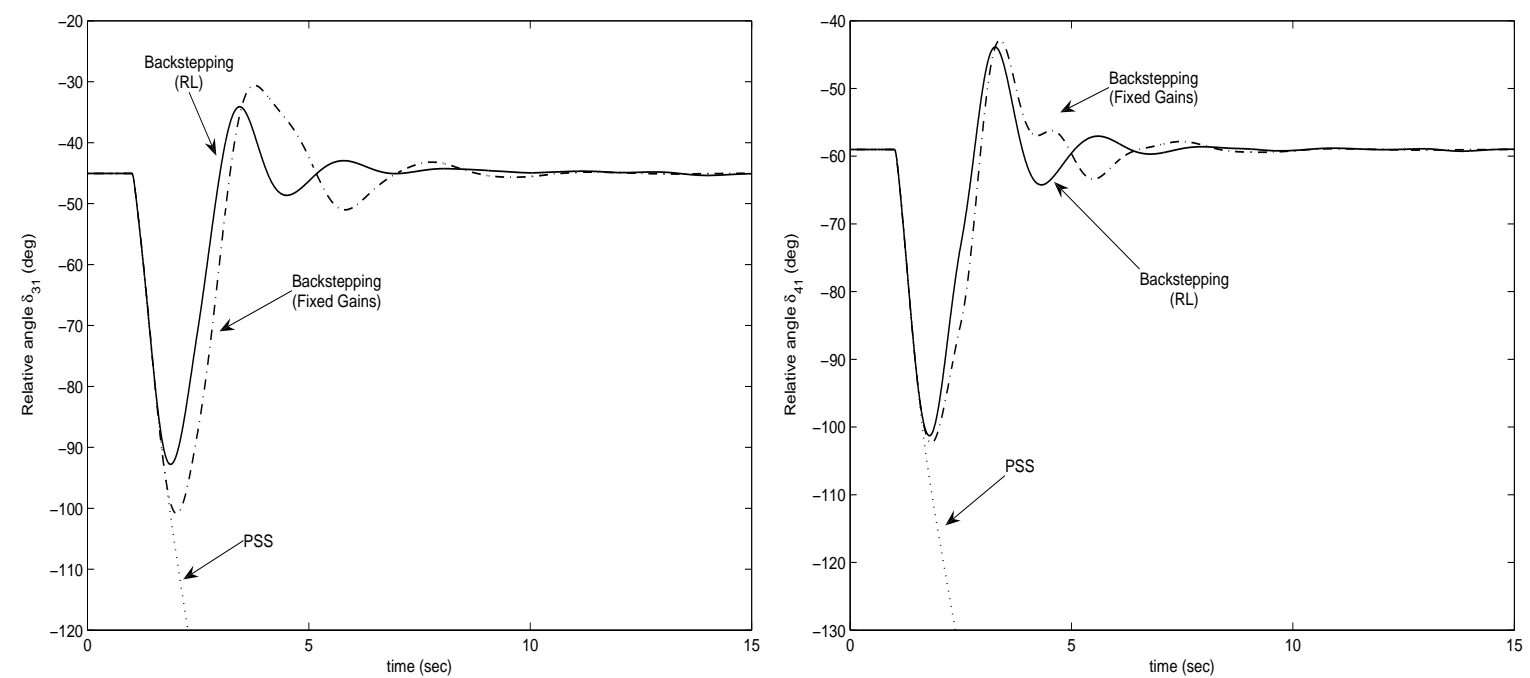

Figure 6.16: Scenario-2 Relative rotor angles $\delta_{31}, \delta_{41}$. Proposed control (solid line), Backstepping with fixed gains (dash-dotted line), PSS (dotted line) 

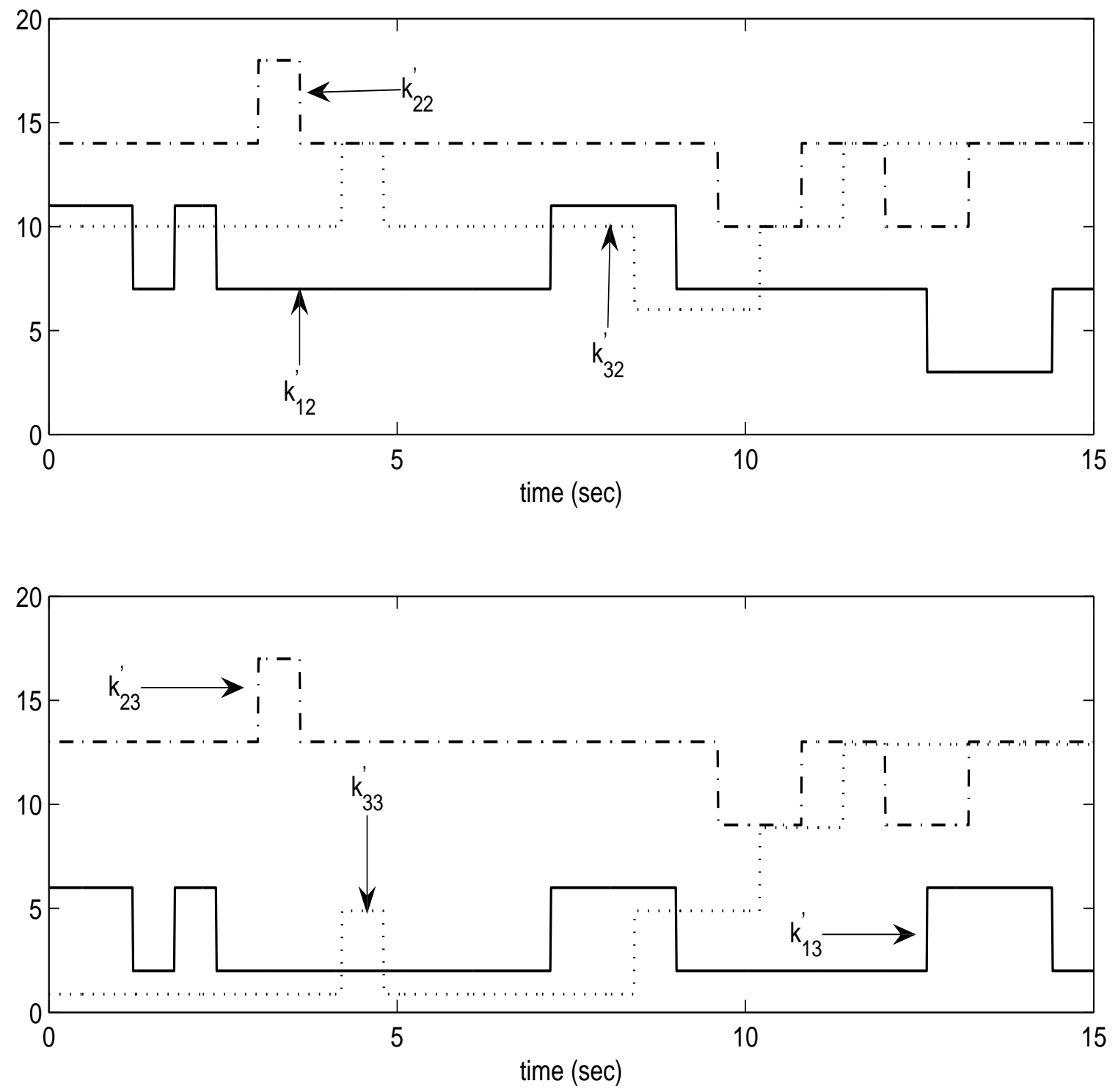

Figure 6.17: Scenario-2 Control gains for generator 2,3 

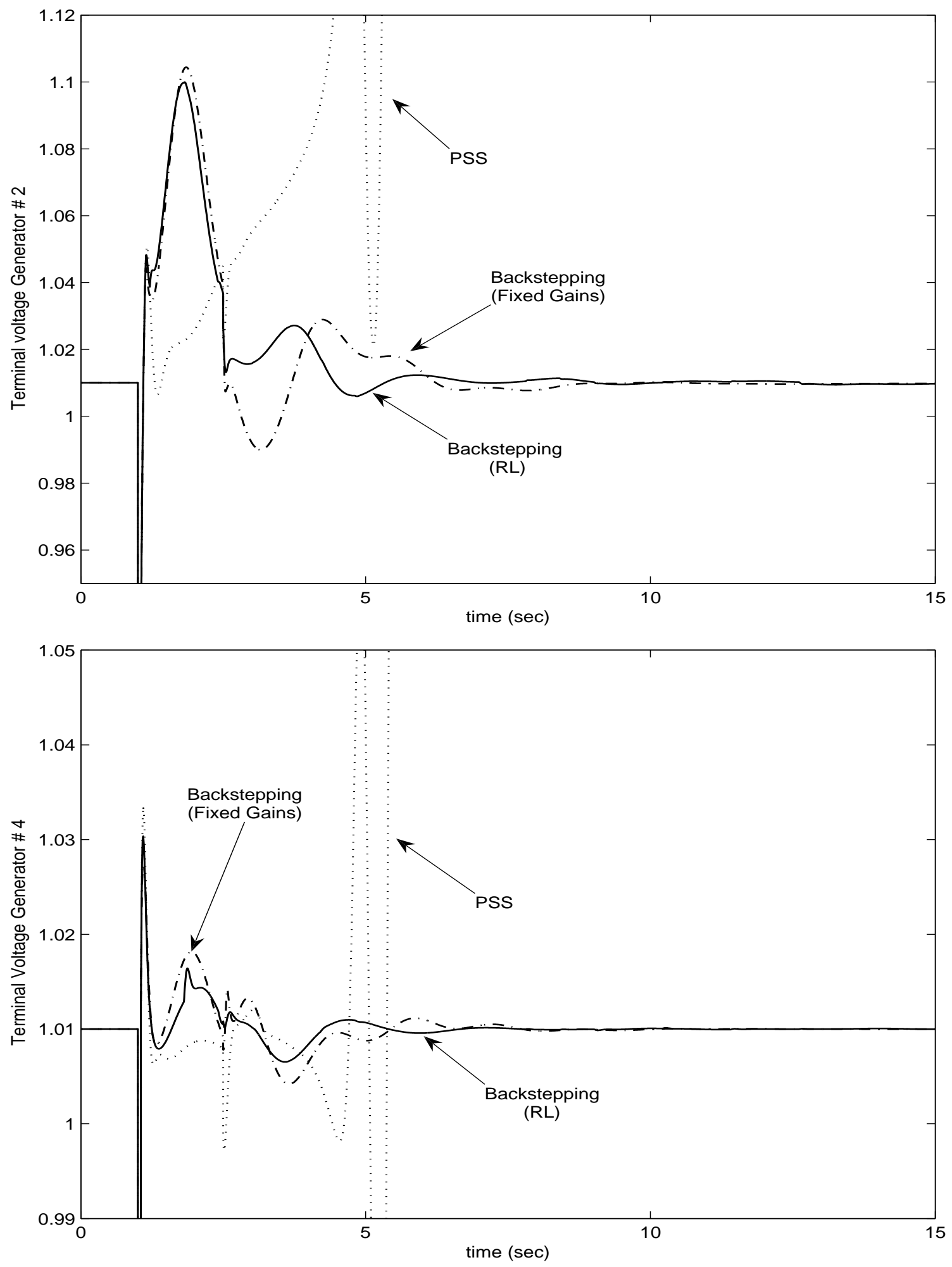

Figure 6.18: Scenario-2 Terminal voltage generator 2, 4 . 

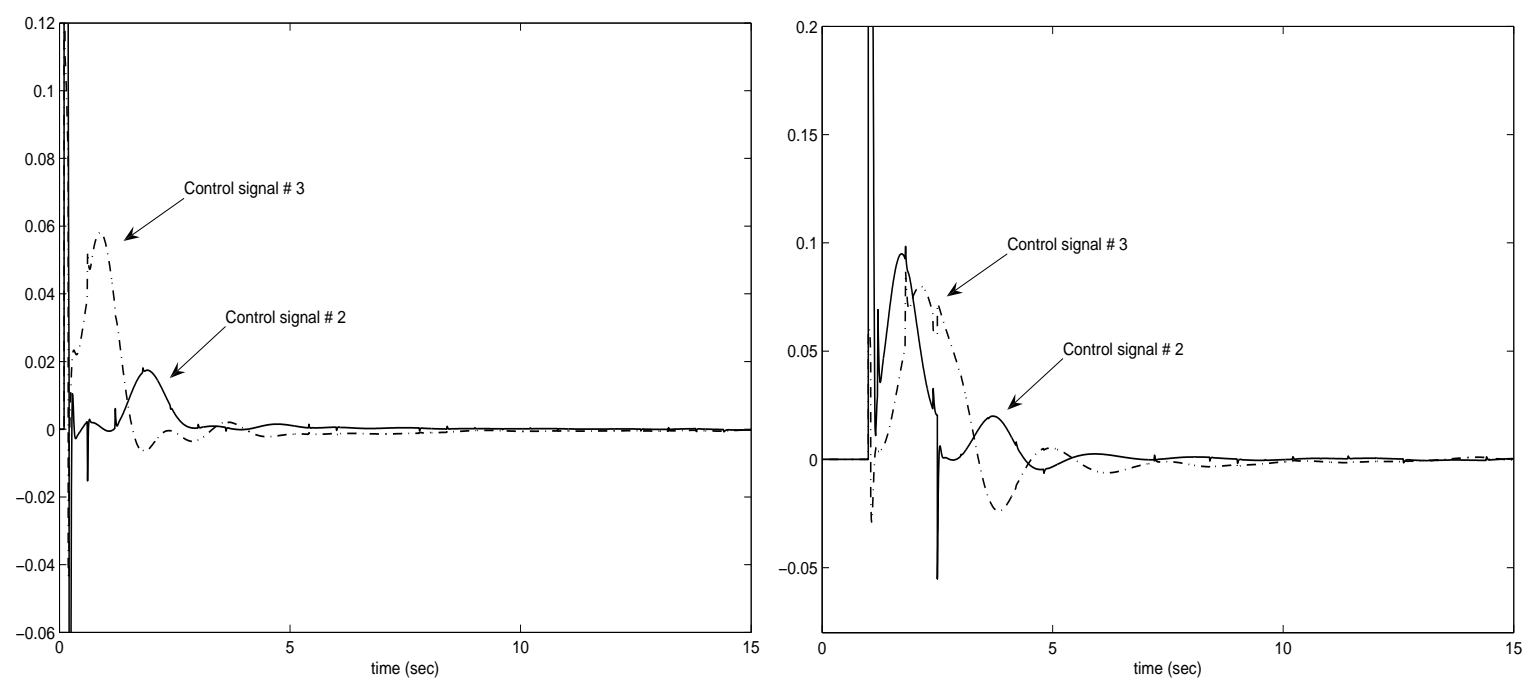

Figure 6.19: Scenario-1 Supplementary control signals for generators 2,3

Results for both cases demonstrate that the reinforcement learning is well suited to be applied in excitation control environment. Supplementary control signals for both scenarios (generators 2,3) are shown in Figures 6.19. Note that for both scenarios the pre-fault and post-fault equilibrium points are similar. For the case where pre-fault and post-fault does not match, for instance removing the transmission line without re closing, there will become an offset in equilibrium point of post-fault which needs to be re-adjusted in backstepping control design. The re-adjustment can be applied in performance evaluation where the transformed states, and tie line powers information are collected (Figure 6.12). In concept of backstepping controller for a excitation control of generator, one can obtain local Lyapunov function and find stabilizing feedback control law. Reinforcement learning algorithm adjusts a closed-loop control rule which is mapping from the system states to control action. System state contains a local Lyapunov function plus an interconnection tie line power. This combination provides broad view of system stability. In principle, the combination of backstepping control and reinforcement learning tries to optimize some mix of stability and performance, and here it suggests a combination of reinforcement learning and backstepping control as a way to implement an advanced control scheme for system stability control. The proposed algorithm for the control is capable withstands severe contingencies in the system in which the controller has not been experienced previously and enhances transient stability of system.

The combination of nonlinear controllers designed thru the backstepping technique with the tuning and adaptation of their gains using reinforcement learning is shown in this section 
as a potential approach to power system stability enhancement. First backstepping controllers are designed, with the gains obtained thru a Particle Swarm Optimization search method, and the interface variables are handled as uncertainties. Then RL progressively learns and adapts the gains to handle a wide range of operating conditions, not covered with backstepping alone. This proposed approach is successfully demonstrated using a two-area four-generator power system. 


\section{Chapter}

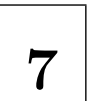

\section{Summary and Conclusions}

\subsection{Completed Work}

This dissertation proposes a backstepping control design to enhance transient stability of power system subject to large disturbances. Two specific classes of controllers have been investigated. First, nonlinear controller is designed for generation units. Generators are considered as subsystems that connect through transmission lines. Generator models are presented in strict feedback form. This form is suitable for applying backstepping control technique. Backstepping controller gains require proper tuning. Particle Swarm Optimization technique is presented and implemented for obtaining these control gains. It is shown through different case studies that tuned controller can improve the transient stability and damp system oscillations in great extent.

To achieve decentralized control, interfaces between generators thru transmission lines are modeled and estimated with two approaches.

I. Interconnection term is considered as noisy measurement of interface that represents the effect of the rest of the system on a generator. The additive nonlinear damping or extended term is added with backstepping control to counteract the effect of disturbance. 
II. Interconnection term is considered as uncertainties. To decouple each generator model so to design a local controller, the effect of the rest of the system on the generator is estimated with a linear estimator whose coefficients are obtained adaptively. This uncertain function is expressed as a polynomial of electric power, and its parameters are obtained by adaptation laws through adaptive backstepping technique.

Second class of controller provides a damping signal for Static Var Compensator. The problem is formulated as an optimization problem with PSO algorithm. The proposed controller requires both local and remote information that are obtained from center of inertia for each area. The widely dispersed signals of power systems are processed and distributed over the entire system. Results on standard benchmark system demonstrate that the SVC damping controller outperforms the lead-lag during severe contingencies in the system.

While these two types of controllers improve the dynamic performance significantly, a coordination of these controllers is even more promising. The proposed coordinated controller is designed based on backstepping technique. The problem is formulated as an optimization problem based on PSO so as to obtain the optimum control gains. By selecting proper objective function during optimization of nonlinear controllers, notable improvement on system performance and optimization are obtained. On the last part, the combination of reinforcement learning and backstepping controller is proposed for generation units. The RL progressively learns and adapts the backstepping gains to handle a wide range of operating conditions, not covered with backstepping alone.

\subsection{Suggestion for Future Work}

The work in this dissertation first concerned on decentralized control for generating units, a topic that has dominated the power system industry for decades. Nevertheless, new technologies allow obtaining of accurate measurements, fast signal processing, and communication using a variety of different combination controllers with information from each area. New control algorithms will be required to ensure the most advantageous overall system response. Despite excellent performance of nonlinear controllers, they are mainly model-based. This requires information from the system, which might not be available always. More effort should be made on non-model based controllers which provide less dependency on system parameters and become independent from specific modeling of the system. A fairly simplistic scheme 
has been introduced in this dissertation for coordination and more advanced controller by combining the reinforcement learner and decentralized backstepping controller for generating units. This approach can be extended among different classes of controllers such as excitation control and FACTS devices.

\subsection{Accomplishments and list of publications}

This section provides lists of research work already done or in progress that is based on parts of this dissertation.

1. A. Karimi, K. Schoder, A. Feliachi, "Decentralized Backstepping Control of Power Systems with Observation Decoupled State Space Method," IEEE PES Power Systems Conference and Exposition PSCE, NewYork, 2004.

Abstract:

This paper proposes a backstepping technique for designing excitation controller to enhance the transient stability of multi-machine power systems under different contingencies, while maintaining terminal voltage at prefault nominal value by adding automatic voltage regulator to controller. The Observation Decoupled State Space (ODSS) is used to obtain the states locally. Two cases are considered, a single machine infinite bus and multi-machine power system.

2. A. Karimi, A. Al-Hinai, K. Schoder, A. Feliachi, "Power system stability enhancement using backstepping controller tuned by particle swarm optimization technique," IEEE Power Engineering Society General Meeting, San Francisco, CA, 2005.

Abstract:

A method for designing controls through the excitation system and particle swarm optimization technique to search for the optimal setting of the controller gains to improve transient stability and damping is presented. Simulation of multi-machine power systems are performed to show the effectiveness of the proposed controller. Comparisons with two other control schemes namely (i) a voltage regulator combined with a power system stabilizer and (ii) excitation controls designed by using the direct feedback linearization (DFL) technique are given to further benchmark the control scheme.

3. A. Karimi, A. Feliachi, "Decentralized extended-backstepping control of power systems," 
IEEE PES Power Engineering Society General Meeting, Montreál, Canada, 2006.

Abstract:

In this paper backstepping controller with additive nonlinear damping term is applied for stability enhancement of multi-machine power systems through excitation control. The decentralized control scheme is practical in systems especially when couplings are handled appropriately. Couplings between generators through transmission lines are modeled and considered as external disturbance through each plant. Controller design, extended-backstepping, is based on a recursive technique to obtain stabilizing control via Lyapunov function. Additive nonlinear damping (extended term) is used to counteracts the effect of disturbance. Controller gains are obtained with particle swarm optimization technique. Results on 50 machines 145 buses show that extended-backstepping controllers effectively stabilize the system for severe contingencies in comparison with conventional power system stabilizer.

4. A. Karimi, A. Feliachi, "PSO-tuned Adaptive Backstepping Control of Power Systems," IEEE PES Power Systems Conference and Exposition PSCE, Atlanta, Ga, 2006.

Abstract:

In this paper, an adaptive backstepping excitation control, tuned using particle swarm optimization (PSO), is designed for stability enhancement of multi-machine power systems. The interconnection terms are considered as uncertain functions of electric power and its derivative whose parameters are adapted using PSO. The proposed technique is illustrated on a 50-machine-145-bus system, which shows that the designed controllers are effective in stabilizing the system under severe contingencies and perform better when compared with conventional power system stabilizers

5. A. Karimi, M. A. Choudhry, A. Feliachi, "PSO-based Evolutionary Optimization for Parameter Identification of an Induction Motor," $39^{\text {th }}$ North American Power Symposium NAPS, Las Cruces, NM, 2007.

\section{Abstract:}

In this paper a particle swarm optimization (PSO) algorithm with a constriction factor is applied to identify the parameters of an induction motor. The variables used to estimate electrical and mechanical parameters are the measured stator currents and voltages. Performance of the identification scheme is demonstrated through simulation 
and compared with parameters obtained with a nonlinear least square technique. The estimated parameters compare well with the actual parameters.

6. A. Karimi, A. Feliachi, M. A. Choudhry, "On an SVC backstepping damping nonlinear controller design for power systems," iREP Symposium Bulk Power System Dynamics and Control-VII., Charleston, SC, 2007.

Abstract:

A damping controller for a Static Var Compensator (SVC) is designed using a backstepping control technique to enhance voltage regulation and power system stability. The damping control problem is formulated as an optimization problem using a detailed model of the power system including static and dynamic loads. A particle swarm optimization (PSO) algorithm is used to obtain the optimal control parameter gains. Nonlinear time domain simulations are carried out to examine the performance of the proposed control technique on a single and a multi machine power system. The results for both systems show that the proposed SVC damping controller is superior to conventional lead-lag design especially under high loading conditions and severe contingencies.

7. A. Karimi, A. Feliachi, "Decentralized Adaptive Backstepping Control of Electric Power Systems," Electric Power Systems Research, Volume 78, Issue 3, March 2008, Pages 484493.

Abstract:

In this paper, a decentralized adaptive backstepping excitation controller, tuned using a Particle Swarm Optimization technique (PSO), is designed for stability enhancement of multi-machine power systems. To achieve decentralization, each machine is modeled as an independent uncertain dynamic subsystem, where the uncertainty is a disturbance that represents the effects of the rest of the system on that particular machine. This disturbance is expressed as a polynomial function of electric power deviation, and its parameters are adapted using PSO. The proposed technique is illustrated with a two-area benchmark power system. This system exhibits inter-area oscillations which are effectively damped with the proposed decentralized controllers under severe contingencies, for which traditional power system stabilizers fail.

8. A. Karimi, M. A. Choudhry, A. Feliachi "Coordinated Backstepping Controls for Power System Stability Enhancement," North American Power Symposium, Calgary, Canada, 
Sept 28 th to 30 th, 2008.

Abstract:

In this paper coordination between backstepping damping controllers for exciters and Static Var Compensators (SVC) is presented. The coordinated design problem of both controllers is cast into an optimization problem which is solved using Particle Swarm Optimization (PSO). Nonlinear simulation results are presented for a two area system to show the effectiveness of the proposed approach.

9. A. Karimi, S. Eftekharnejad, A. Feliachi, Reinforcement Learning based Backstepping Control of Power System Oscillations, Electric Power Systems Research, under review.

10. A. Feliachi, K. Schoder, S. Eftekharnejad, A. Karimi, Intelligent Techniques for Power System Transmission, under review Book Chapter 2008. 


\section{APPENDIX}

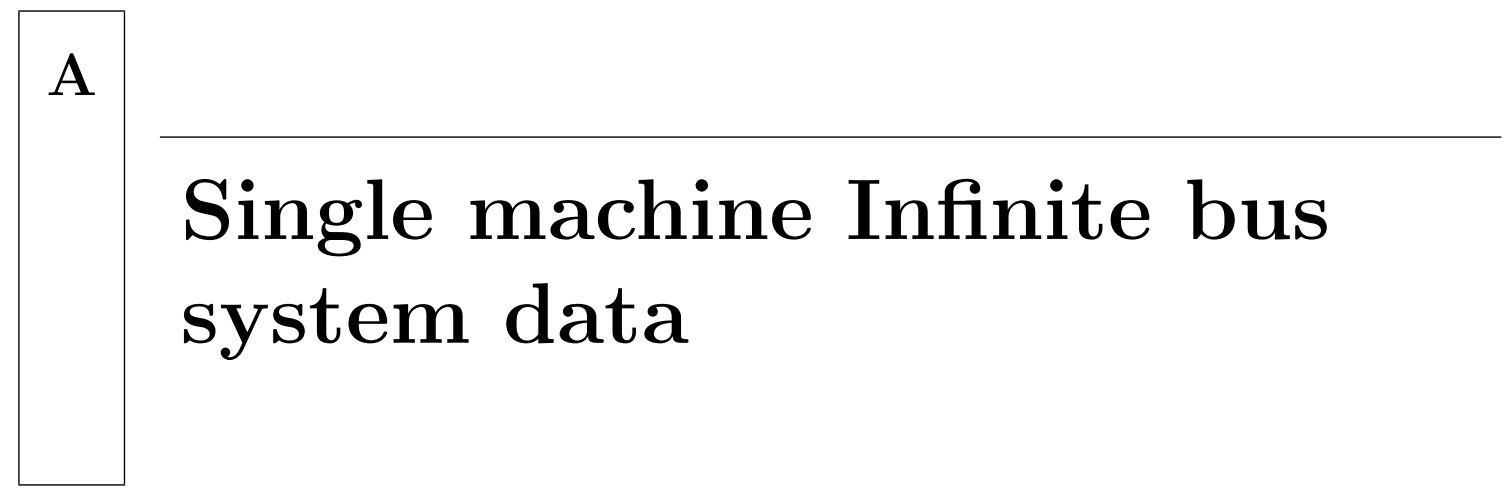

$\%$ Single-machine infinite bus example

$\%$ Base value

basmva $=100$;

$\%$ bus data format

$\%$ bus :

$\%$ col1 number

$\%$ col2 voltage magnitude(pu)

$\%$ col3 voltage angle(degree)

$\%$ col4 p_gen (pu)

$\%$ col5 q-gen (pu),

$\%$ col6 p_load $(\mathrm{pu})$

$\% \operatorname{col} 7$ q_load (pu)

$\%$ col8 G shunt(pu)

$\%$ col9 B shunt (pu)

$\%$ col10 bus_type

$\% \quad$ bus_type -1 , swing bus

$\% \quad-2$, generator bus (PV bus)

$\% \quad-3$, load bus (PQ bus)

$\%$ col11 q-gen_max (pu) 

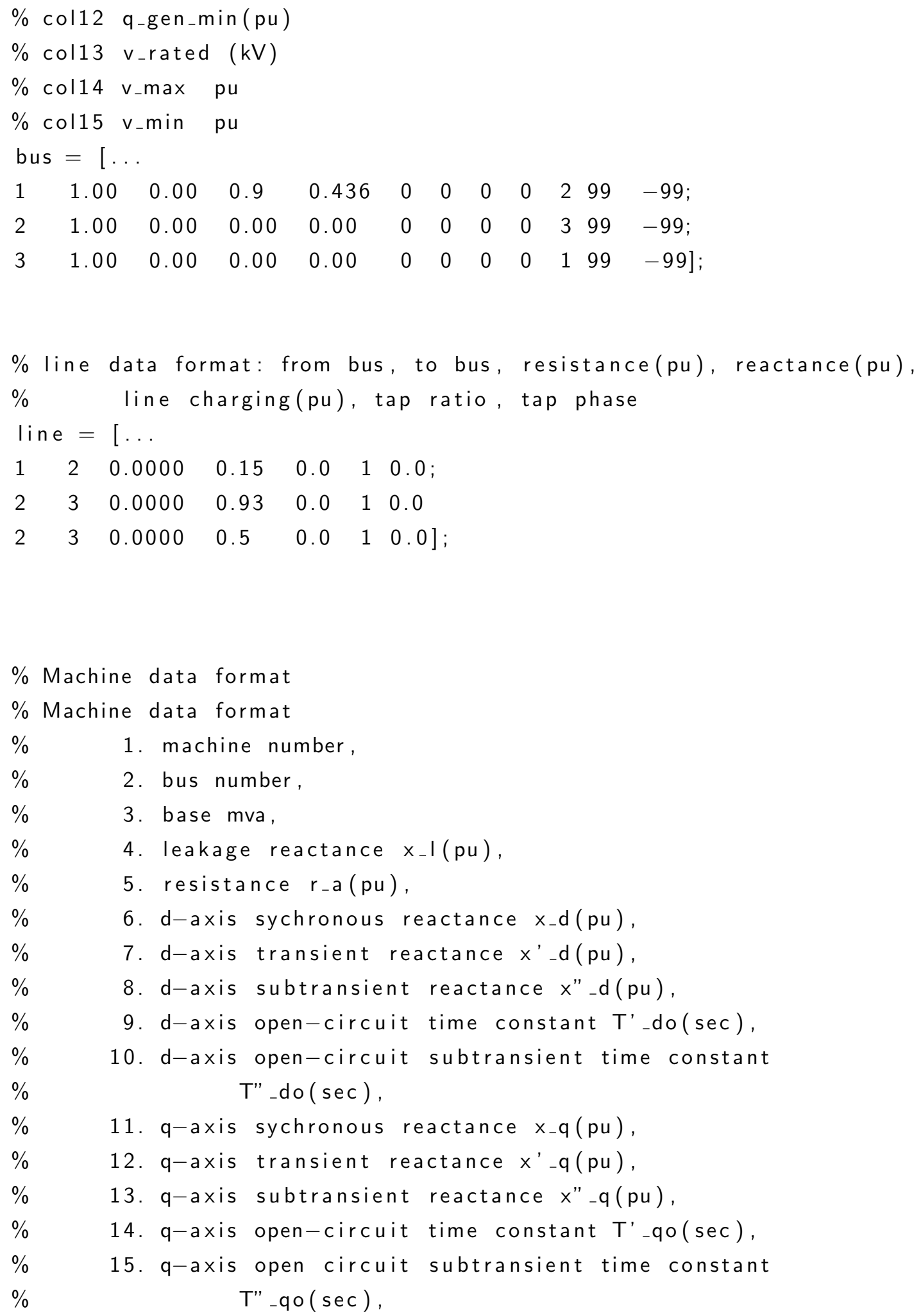


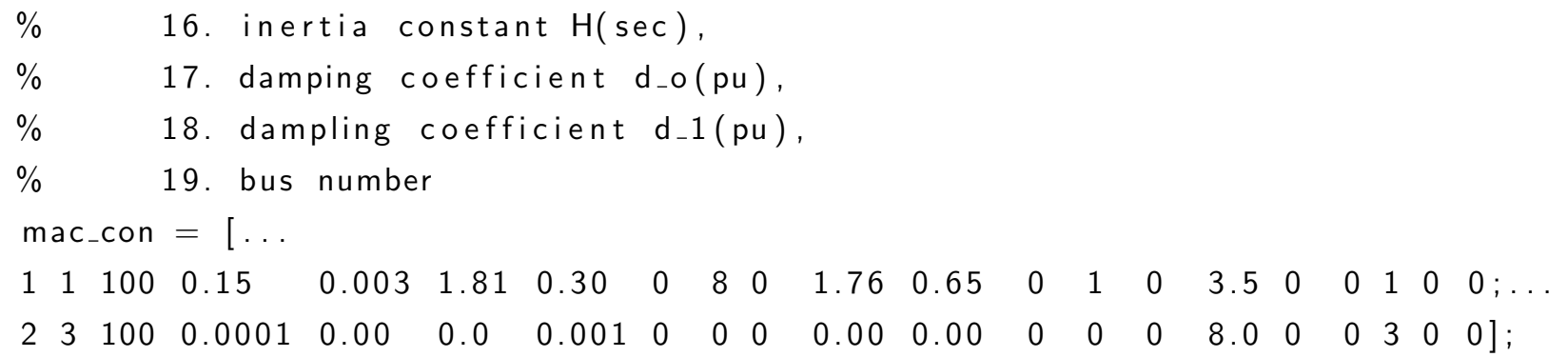

$\%$ Define ifinite bus ibus_con $=\left[\begin{array}{ll}0 & 1\end{array}\right]$; 
$\%$ col4 washout (sec.)

$\%$ col5 lead T_1

$\%$ col6 lag T_2

\%col7 lead T_3

\%col8 lag T_4

$\% \operatorname{col} 9$ V-max (pu)

$\% \operatorname{col} 10 \mathrm{~V}-\min (\mathrm{pu})$

pss_con $=\left[\begin{array}{llllllllll}1 & 1 & 13.7750 & 1.410 & 0.154 & 0.033 & 0.154 & 0.033 & 0.2 & -0.2\end{array}\right]$; 


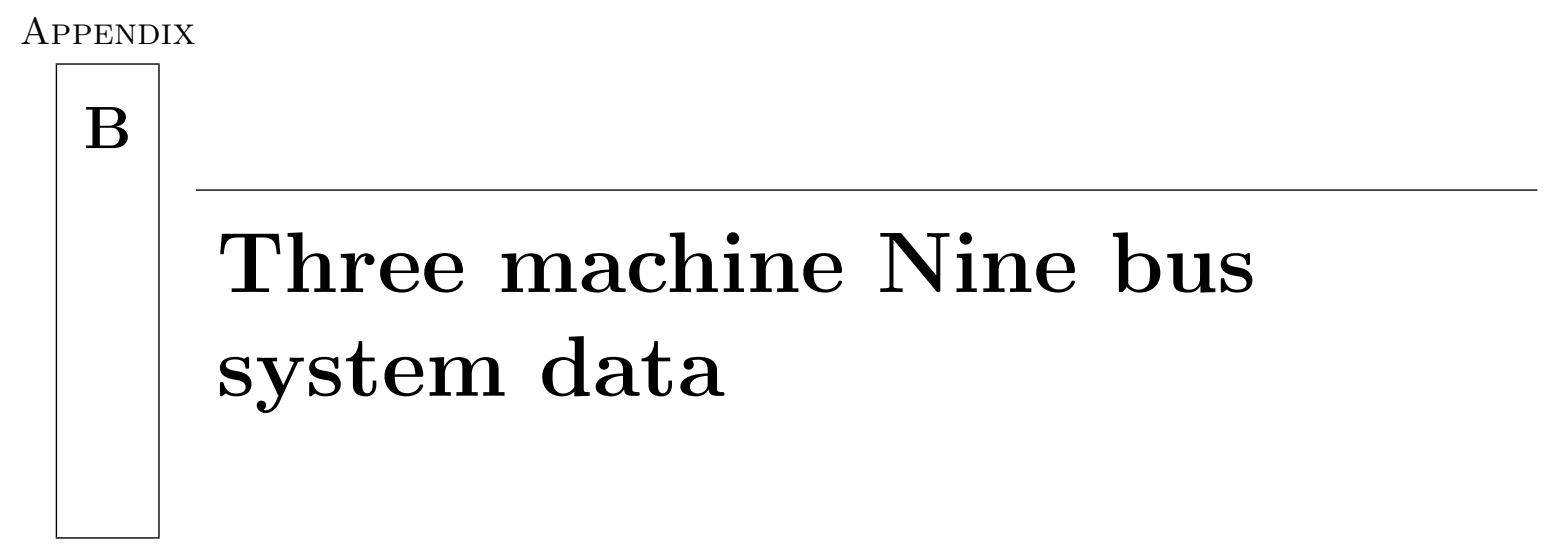

Data file for the three machine nine bus

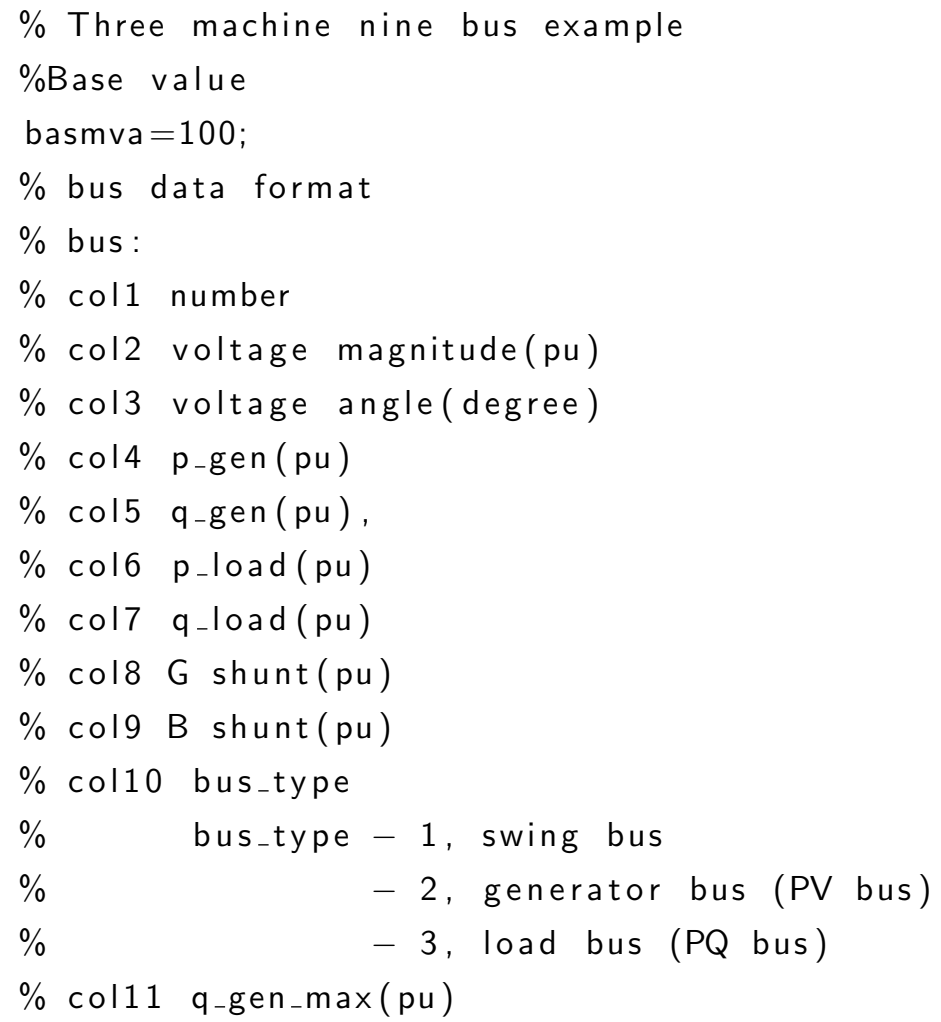




\% col12 q_gen_min $(\mathrm{pu})$
$\%$ col13 v_rated $(\mathrm{kV})$
$\%$ col14 v_max pu
$\%$ col15 v_min $\mathrm{pu}$
bus $=$ [

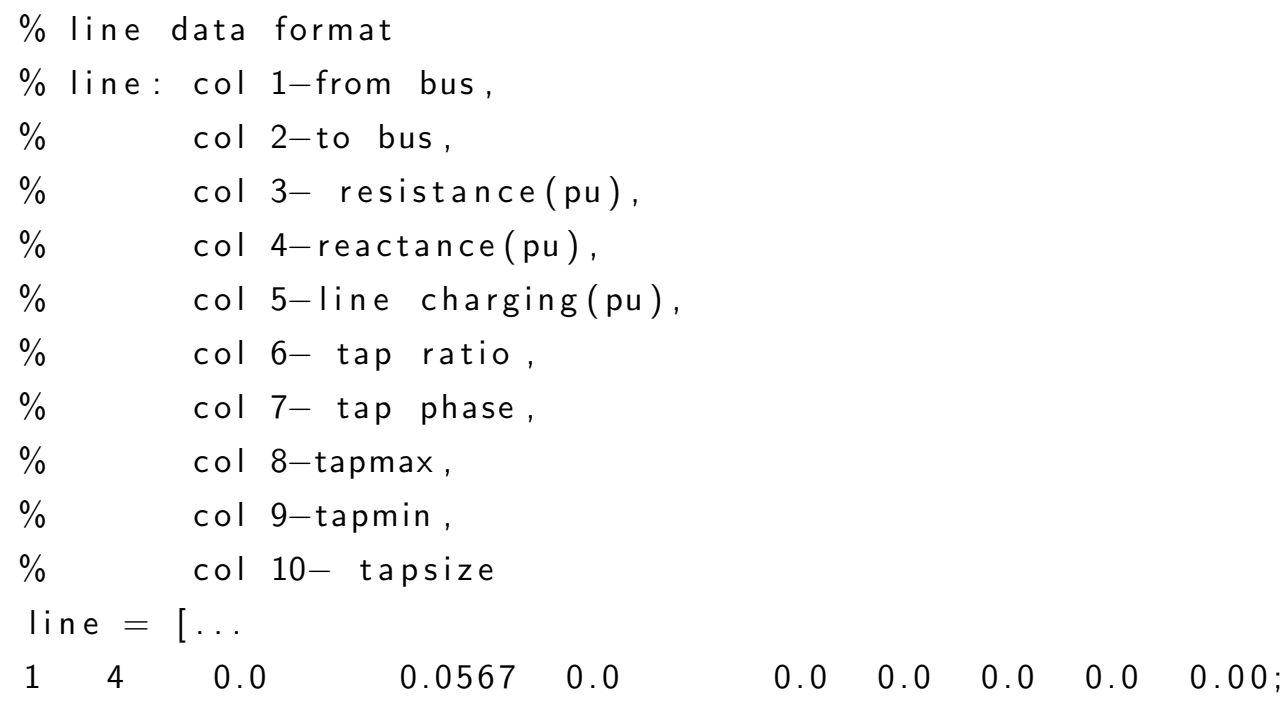




$\begin{array}{llllllllll}2 & 7 & 0.0 & 0.0625 & 0.0 & 0.0 & 0.0 & 0.0 & 0.0 & 0.00 ; \\ 3 & 9 & 0.0 & 0.0586 & 0.0 & 0.0 & 0.0 & 0.0 & 0.0 & 0.00 ; \\ 4 & 5 & 0.01 & 0.085 & 0.088 * 2 & 0.0 & 0.0 & 0.0 & 0.0 & 0.00 ; \\ 4 & 6 & 0.017 & 0.092 & 0.079 * 2 & 0.0 & 0.0 & 0.0 & 0.0 & 0.00 ; \\ 5 & 7 & 0.032 & 0.161 & 0.153 * 2 & 0.0 & 0.0 & 0.0 & 0.0 & 0.00 ; \\ 6 & 9 & 0.039 & 0.170 & 0.179 * 2 & 0.0 & 0.0 & 0.0 & 0.0 & 0.00 ; \\ 7 & 8 & 0.0085 & 0.072 & 0.0745 * 2 & 0.0 & 0.0 & 0.0 & 0.0 & 0.00 ; \\ 8 & 9 & 0.0119 & 0.1008 & 0.1045 * 2 & 0.0 & 0.0 & 0.0 & 0.0 & 0.00] ; ;\end{array}$

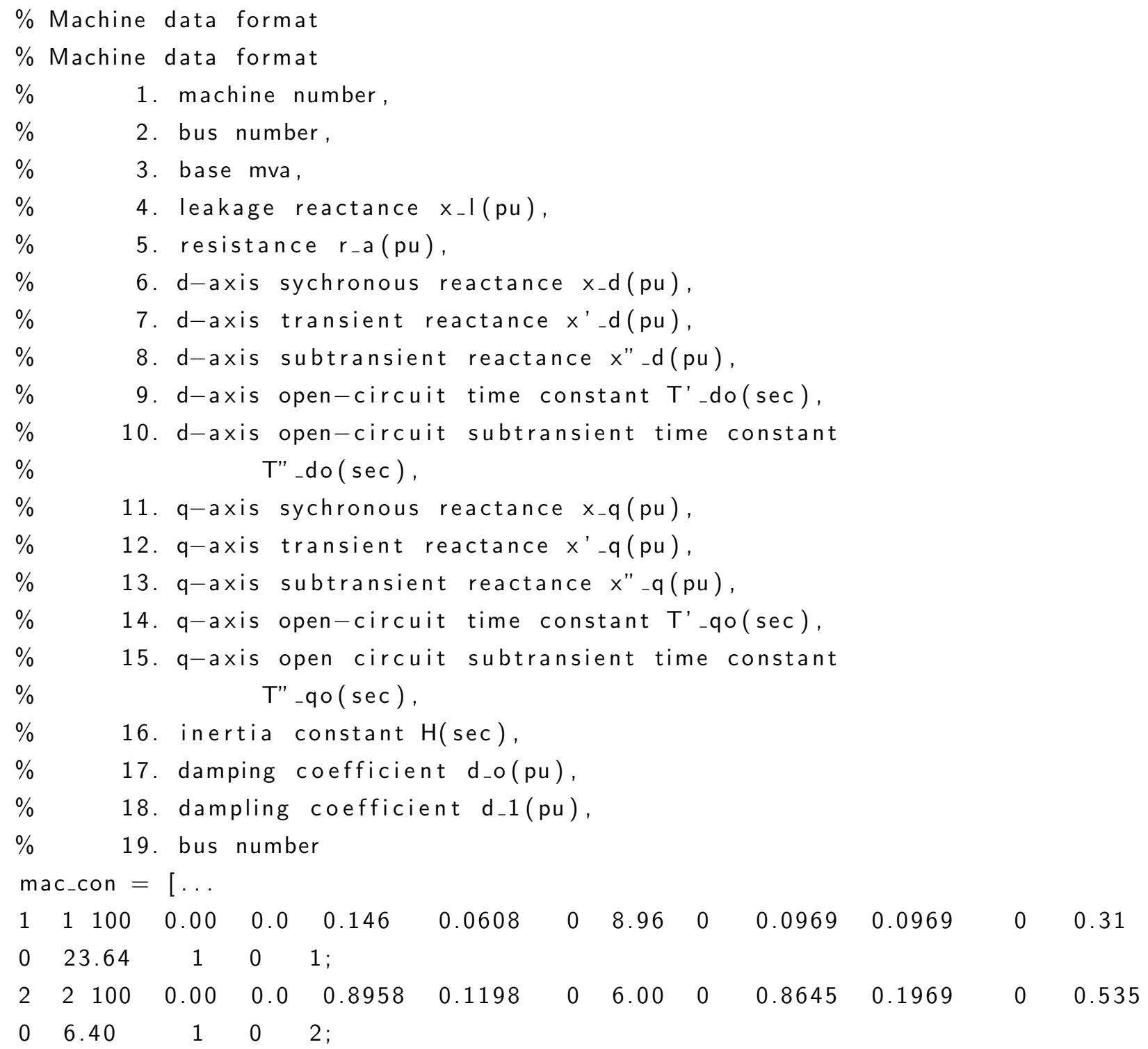




$\begin{array}{lllcllllllllll}3 & 3 & 100 & 0.00 & 0.0 & 1.3125 & 0.1813 & 0 & 5.89 & 0 & 1.2578 & 0.2500 & 0 & 0.600 \\ 0 & 3.01 & 1 & 0 & 3] ;\end{array}$

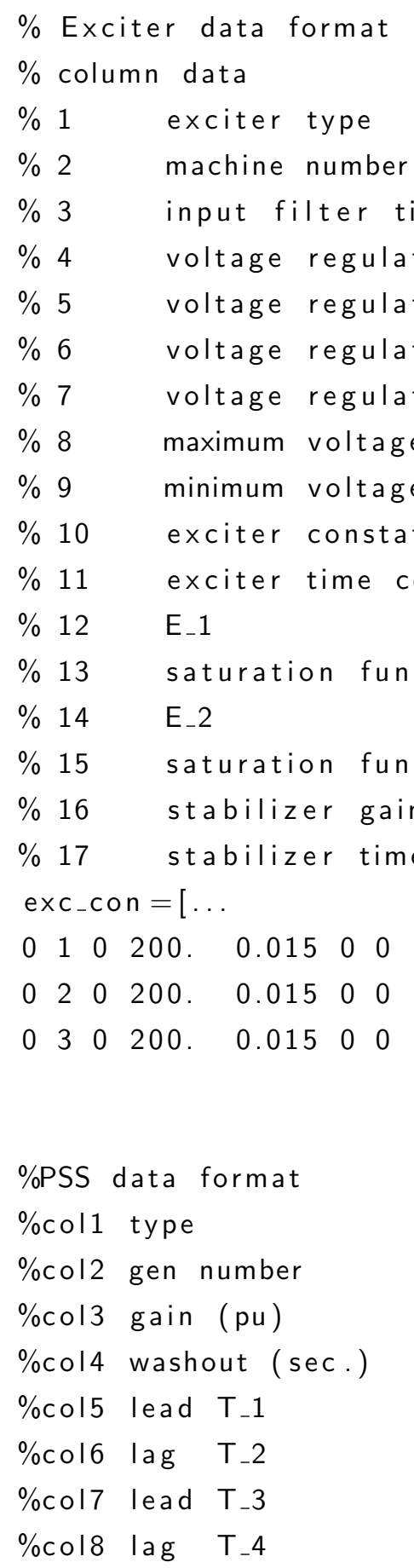


$\% \operatorname{col} 9 \quad \mathrm{~V}-\max (\mathrm{pu})$

$\% \operatorname{col} 10 \mathrm{~V}-\min (\mathrm{pu})$

pss_con $=[\ldots$

$\begin{array}{llllllllll}1 & 1 & 13.7750 & 1.410 & 0.154 & 0.033 & 0.154 & 0.033 & 0.2 & -0.2 \text {; }\end{array}$

$\begin{array}{llllllllll}1 & 2 & 13.7750 & 1.410 & 0.154 & 0.033 & 0.154 & 0.033 & 0.2 & -0.2 \text {; }\end{array}$

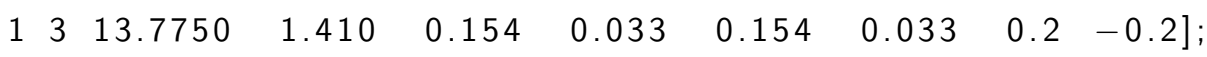

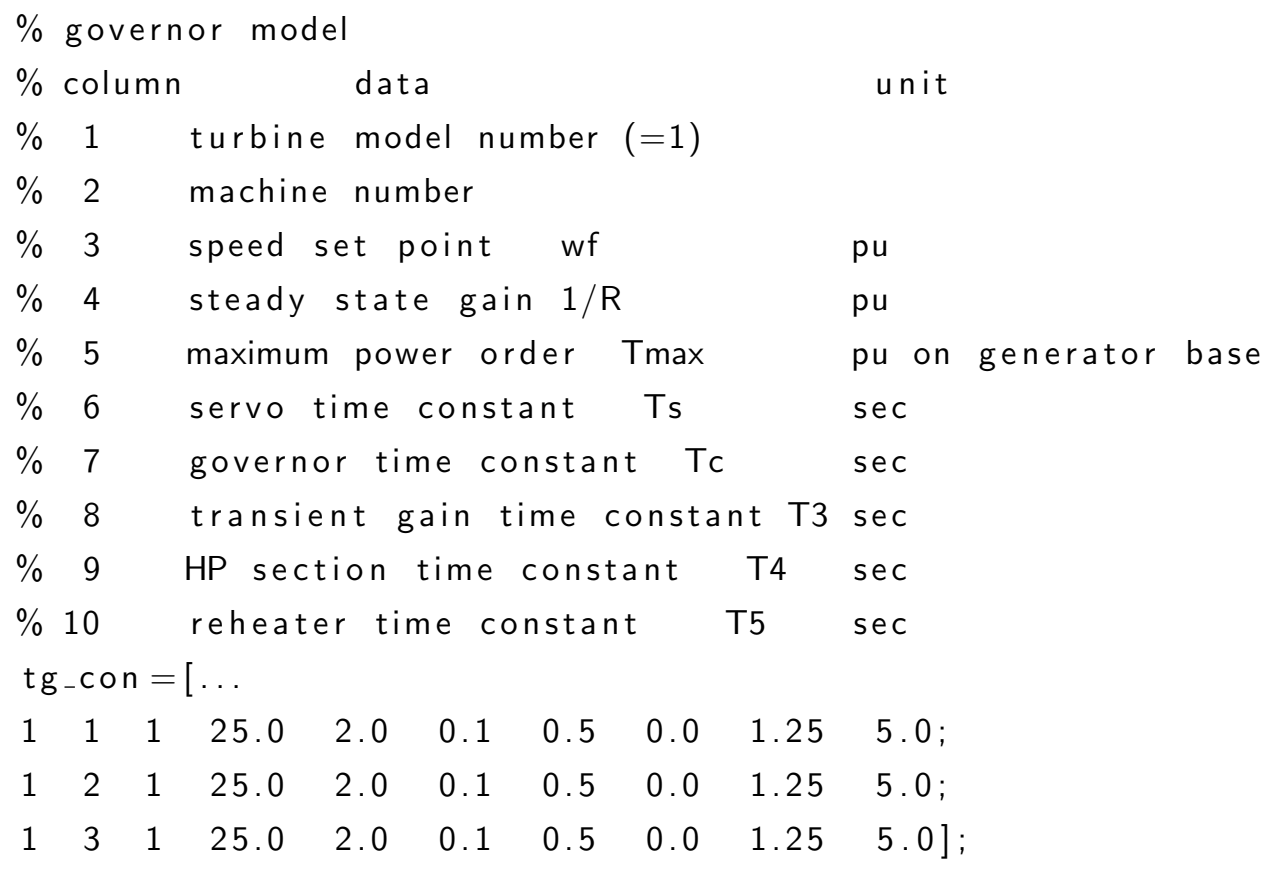



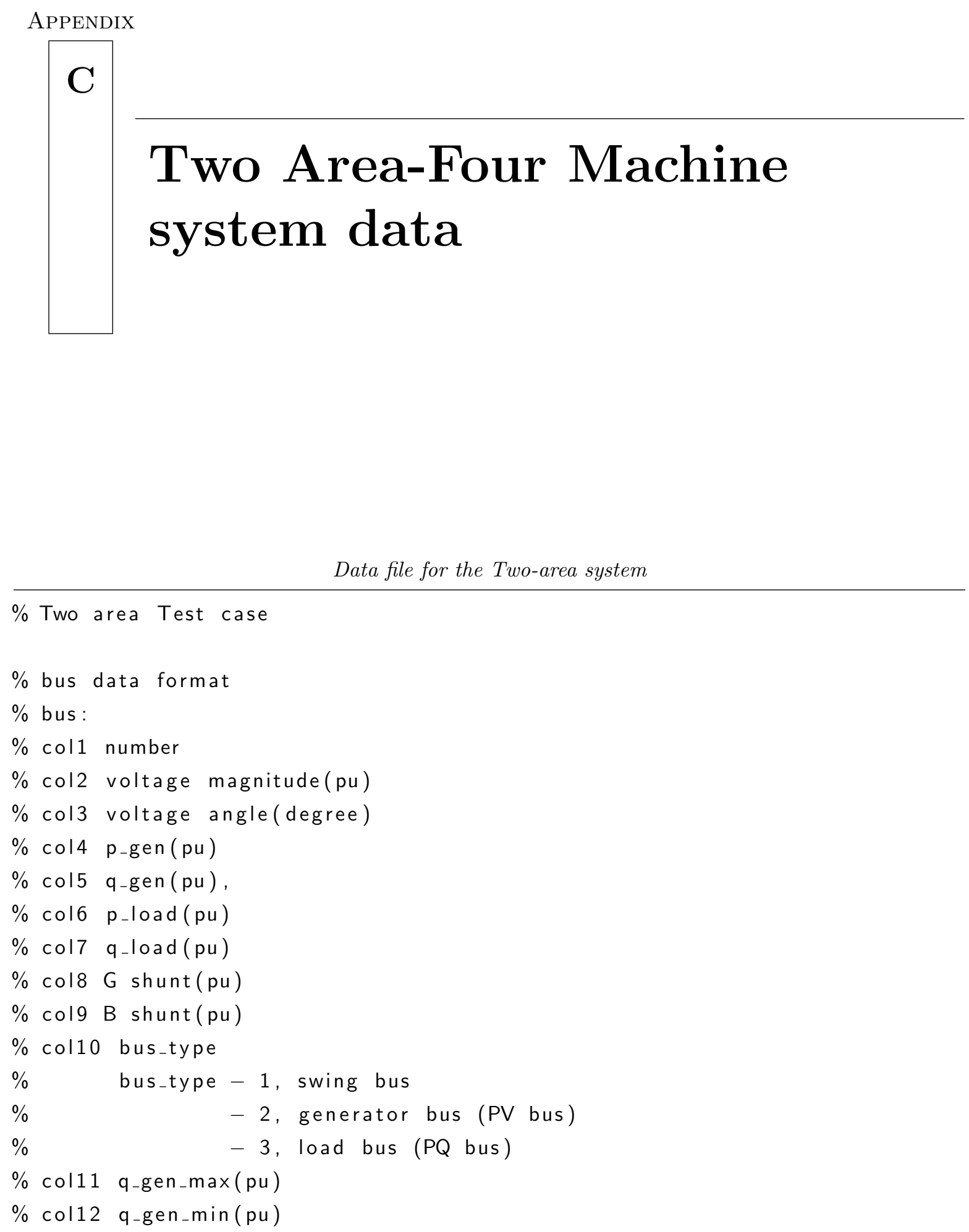


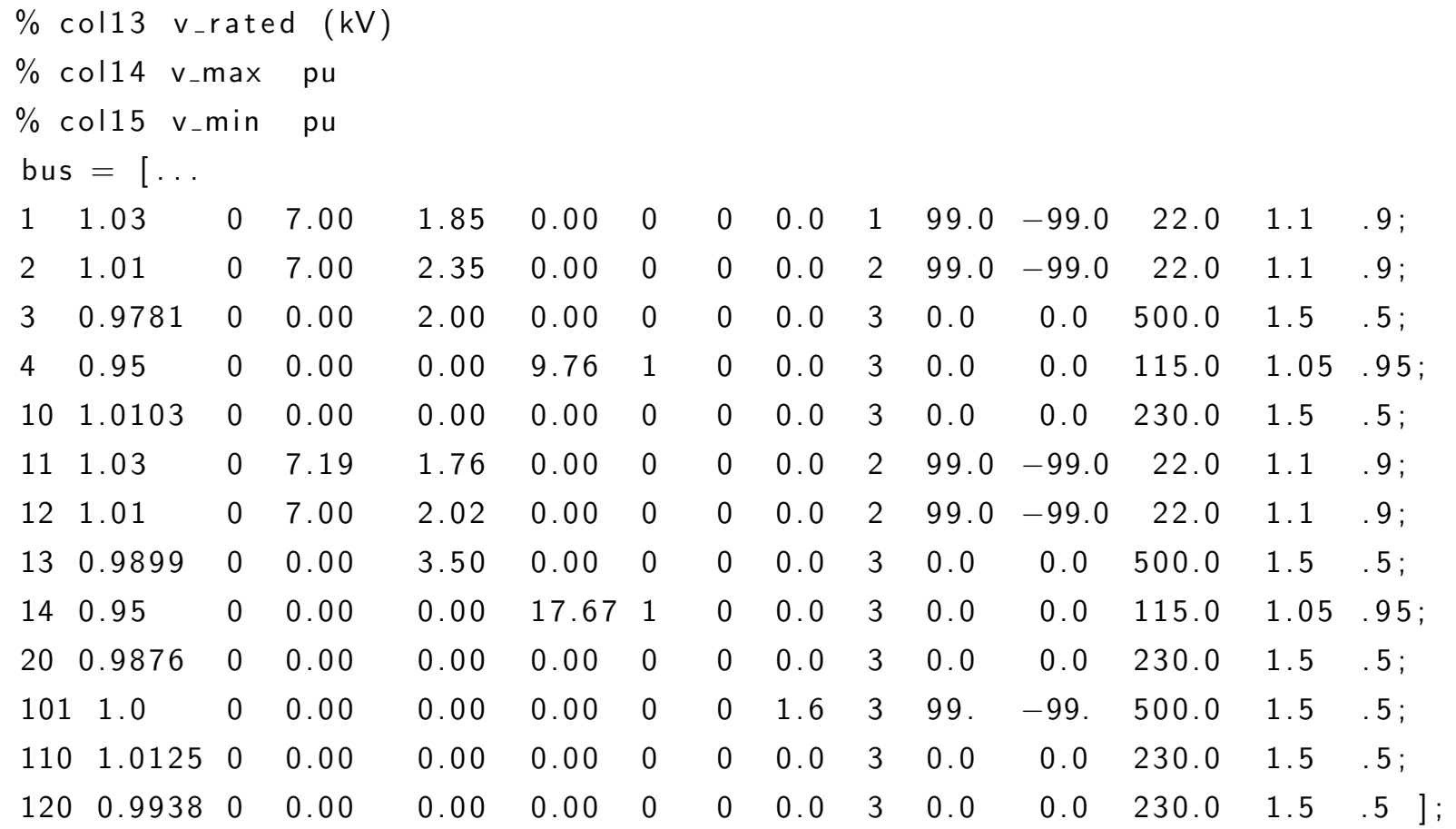

$\%$ line data format: from bus, to bus, resistance(pu), reactance(pu), $\% \quad$ line charging(pu), tap ratio, tap phase, tapmax, tapmin, tapsize line $=[\ldots$

$\begin{array}{lcllllllll}1 & 10 & 0.0 & 0.0167 & 0.00 & 1 & 0.0 . & 0 . & 0 . ; \\ 2 & 20 & 0.0 & 0.0167 & 0.00 & 1 & 0 . & 0 . & 0 . & 0 . ; \\ 10 & 20 & 0.0025 & 0.025 & 0.0437 & 1 & 0 . & 0 . & 0 . & 0 . ; \\ 20 & 3 & 0.001 & 0.0100 & 0.0175 & 1 & 0 . & 0 . & 0 . & 0 . ; \\ 3 & 4 & 0.0 & 0.005 & 0.00 & 1 & 0 . & 1.2 & 0.8 & 0.05 ; \\ 3 & 101 & 0.011 & 0.110 & 0.1925 & 1 & 0 . & 0 . & 0 . & 0 . ; \\ 3 & 101 & 0.011 & 0.110 & 0.1925 & 1 & 0 . & 0 . & 0 . & 0 . ; \\ 101 & 13 & 0.011 & 0.11 & 0.1925 & 1 & 0 . & 0 . & 0 . & 0 . ; \\ 101 & 13 & 0.011 & 0.11 & 0.1925 & 1 & 0 . & 1.2 & 0.8 & 0.05 ; \\ 13 & 120 & 0.001 & 0.01 & 0.0175 & 1 & 0 . & 0 . & 0 . & 0 . ; \\ 120 & 110 & 0.0025 & 0.025 & 0.0437 & 1 & 0 . & 0 . & 0 . & 0 . ; \\ 11 & 110 & 0.0 & 0.0167 & 0.0 & 1 & 0 . & 0 . & 0 . & 0 . ; \\ 12 & 120 & 0.0 & 0.0167 & 0.0 & 1 & 0.0 . & 0 . & 0 .] ;\end{array}$

$\%$ Machine data format 


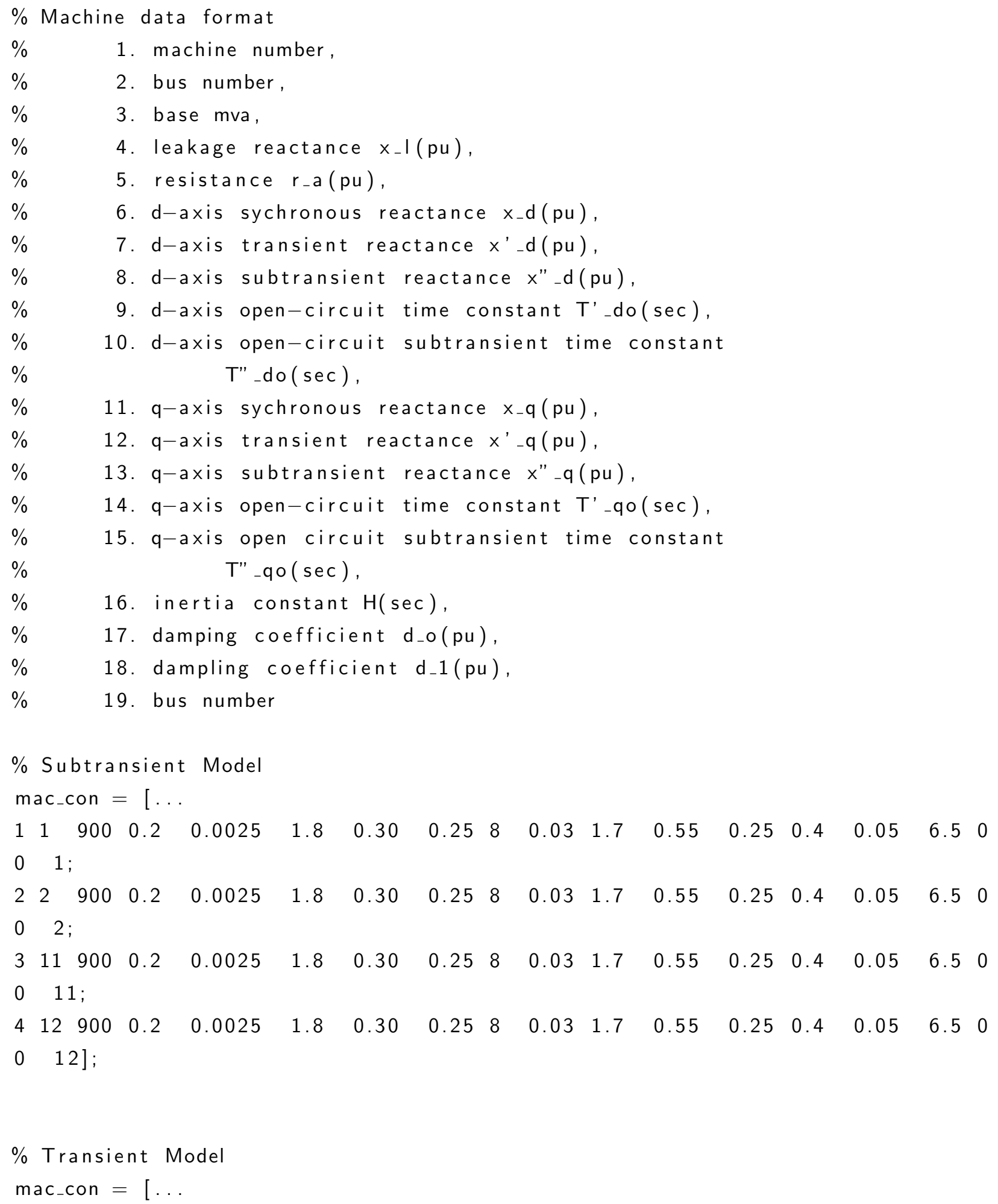




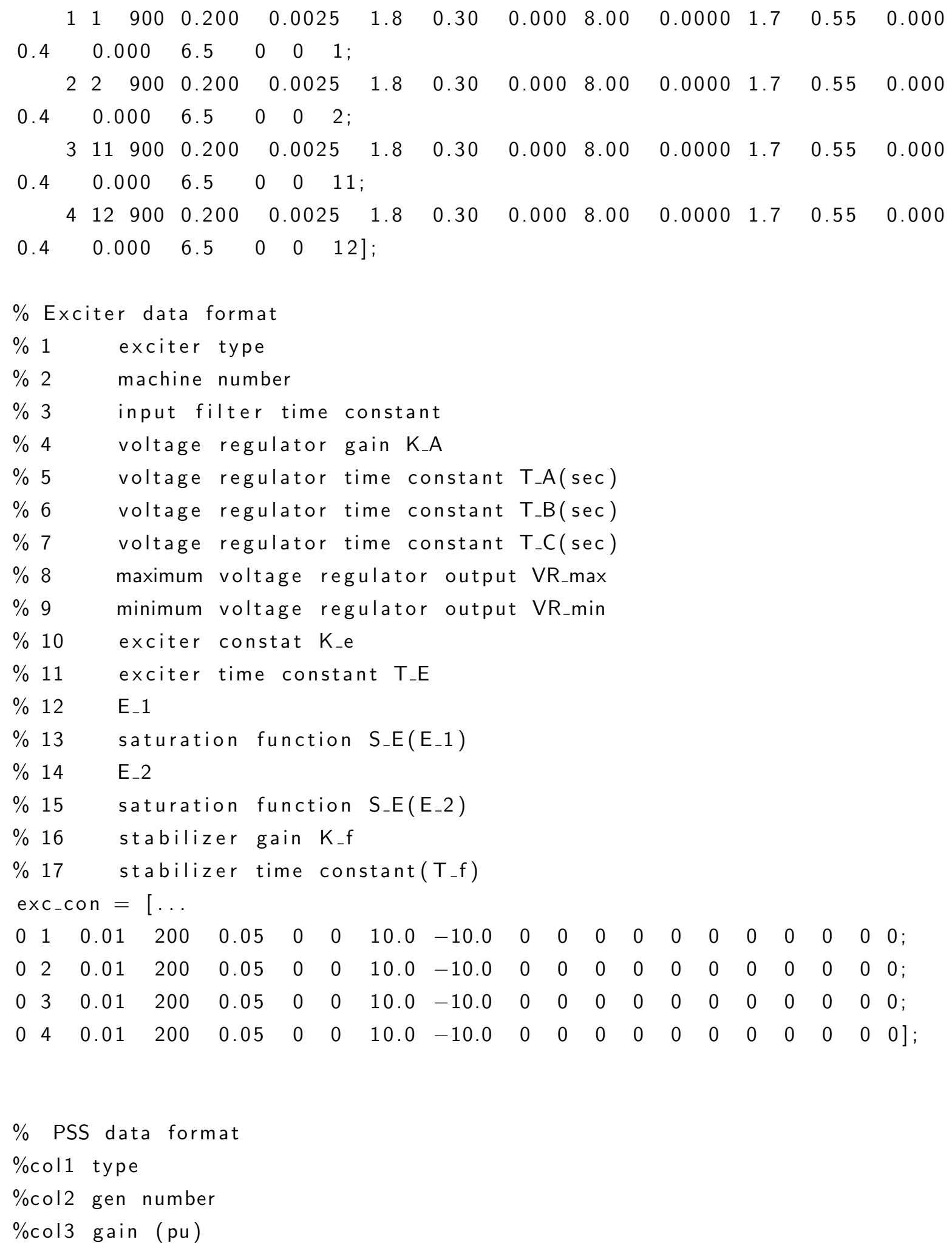


$\%$ col4 washout (sec.)

\%col5 lead T_1

$\%$ col6 lag T_2

\%col7 lead T_3

\%col8 lag T_4

$\% \operatorname{col} 9$ V-max (pu)

\%col10 V-min (pu)

pss_con $=[\ldots$

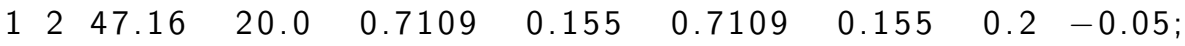

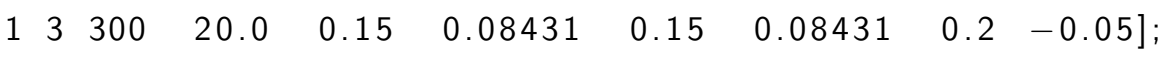

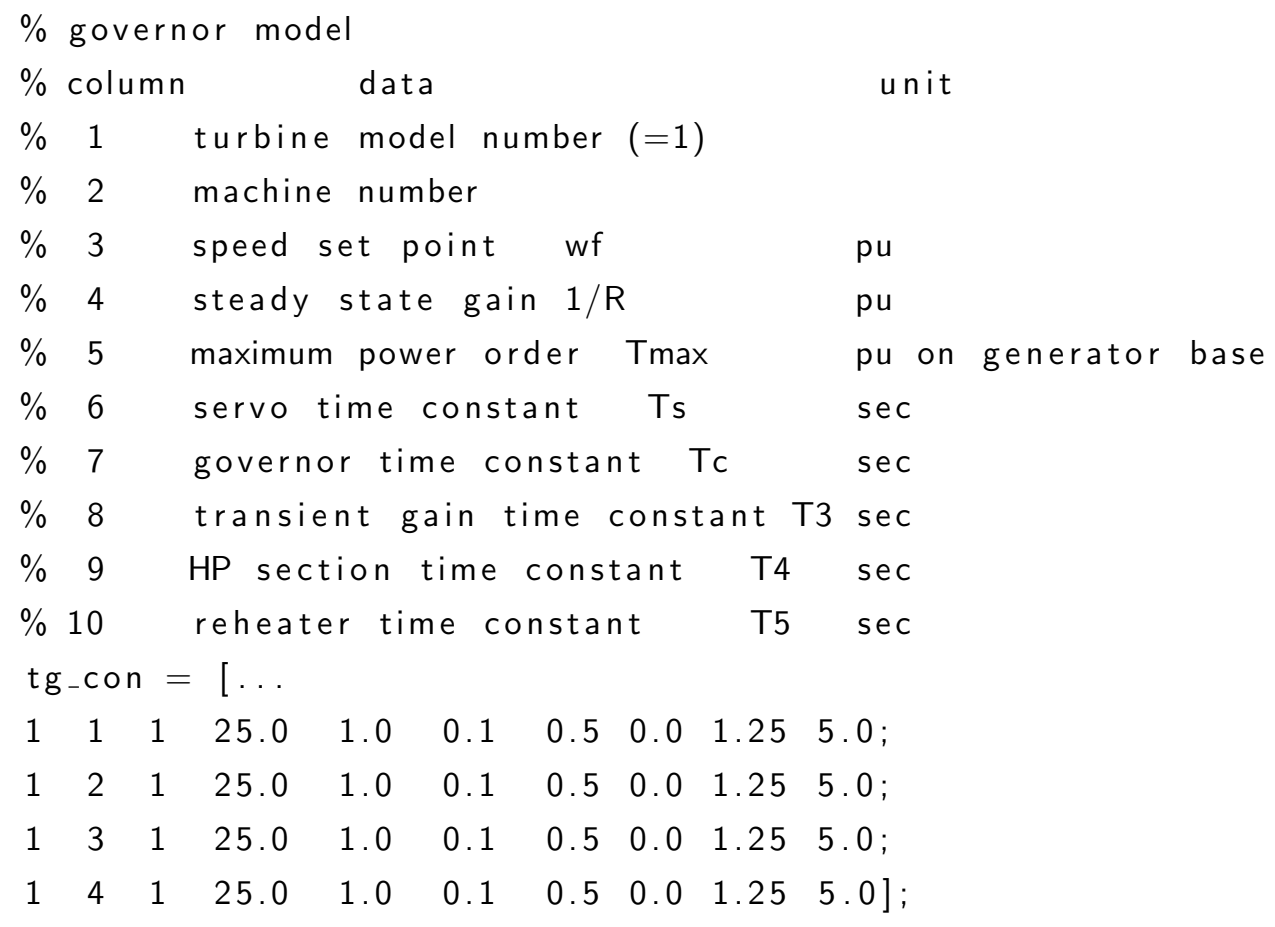




\section{ApPENDIX}

\section{D \\ Fifty Machine system data}

Machine and Exciter data file for the fifty machine system

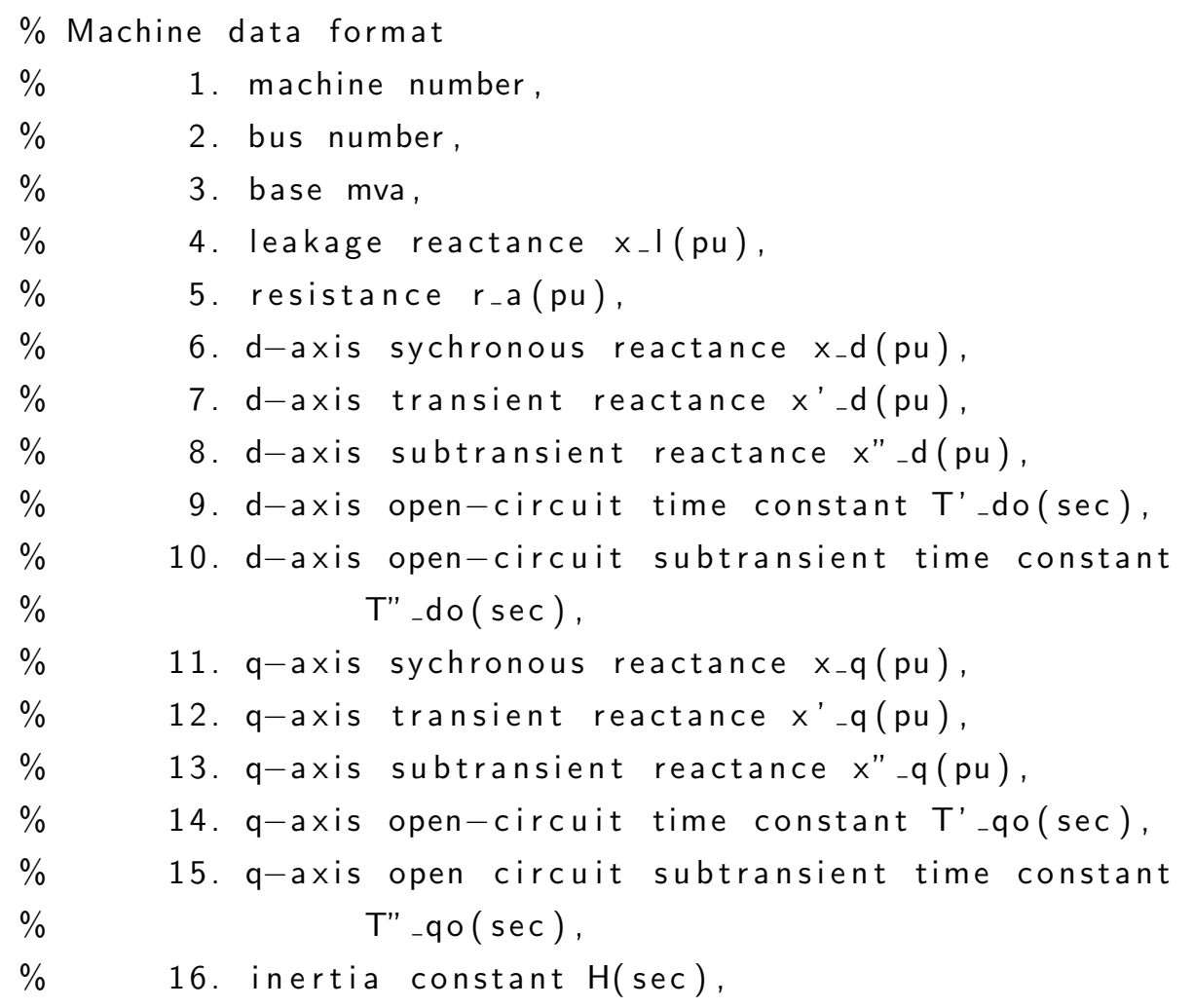




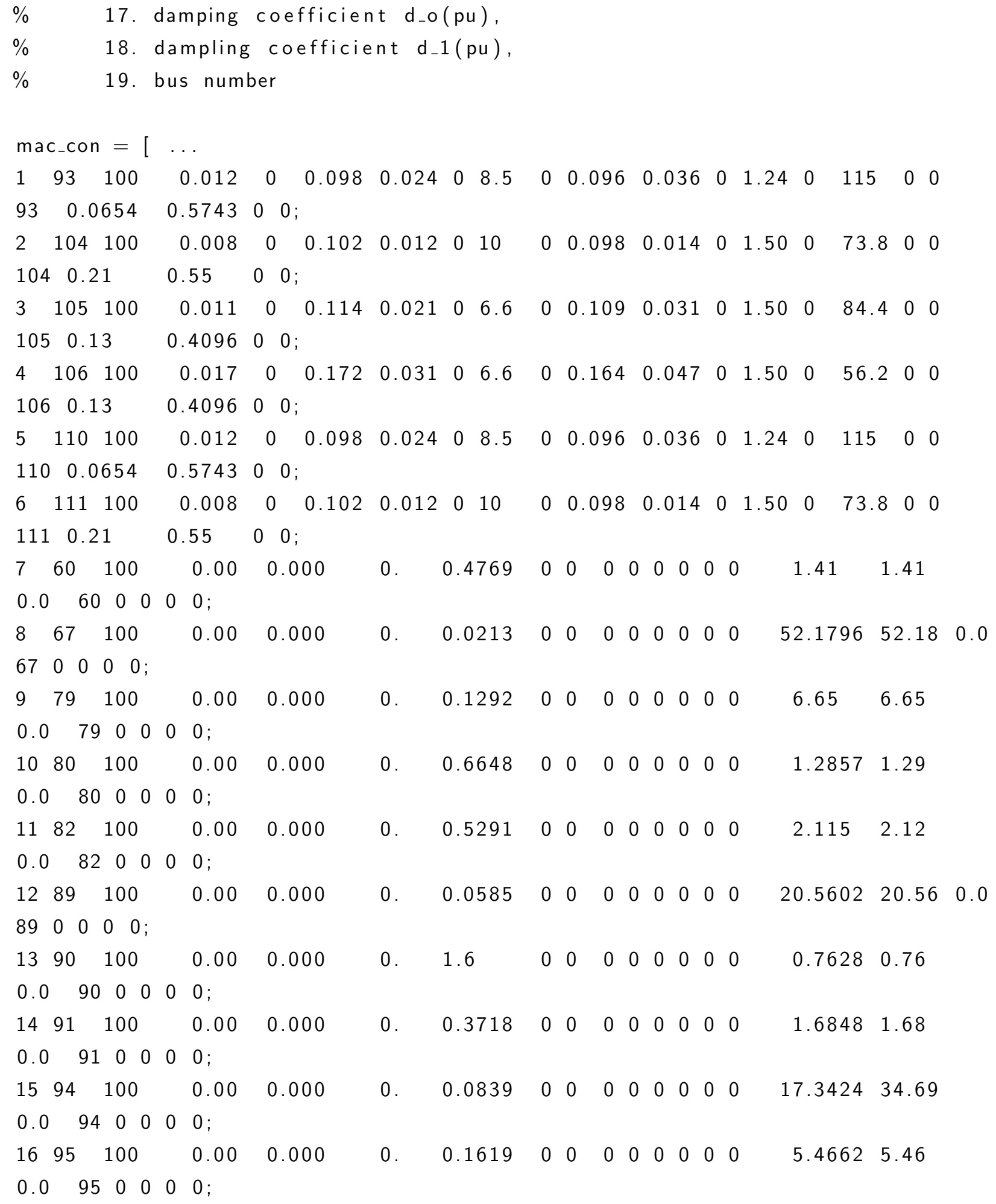




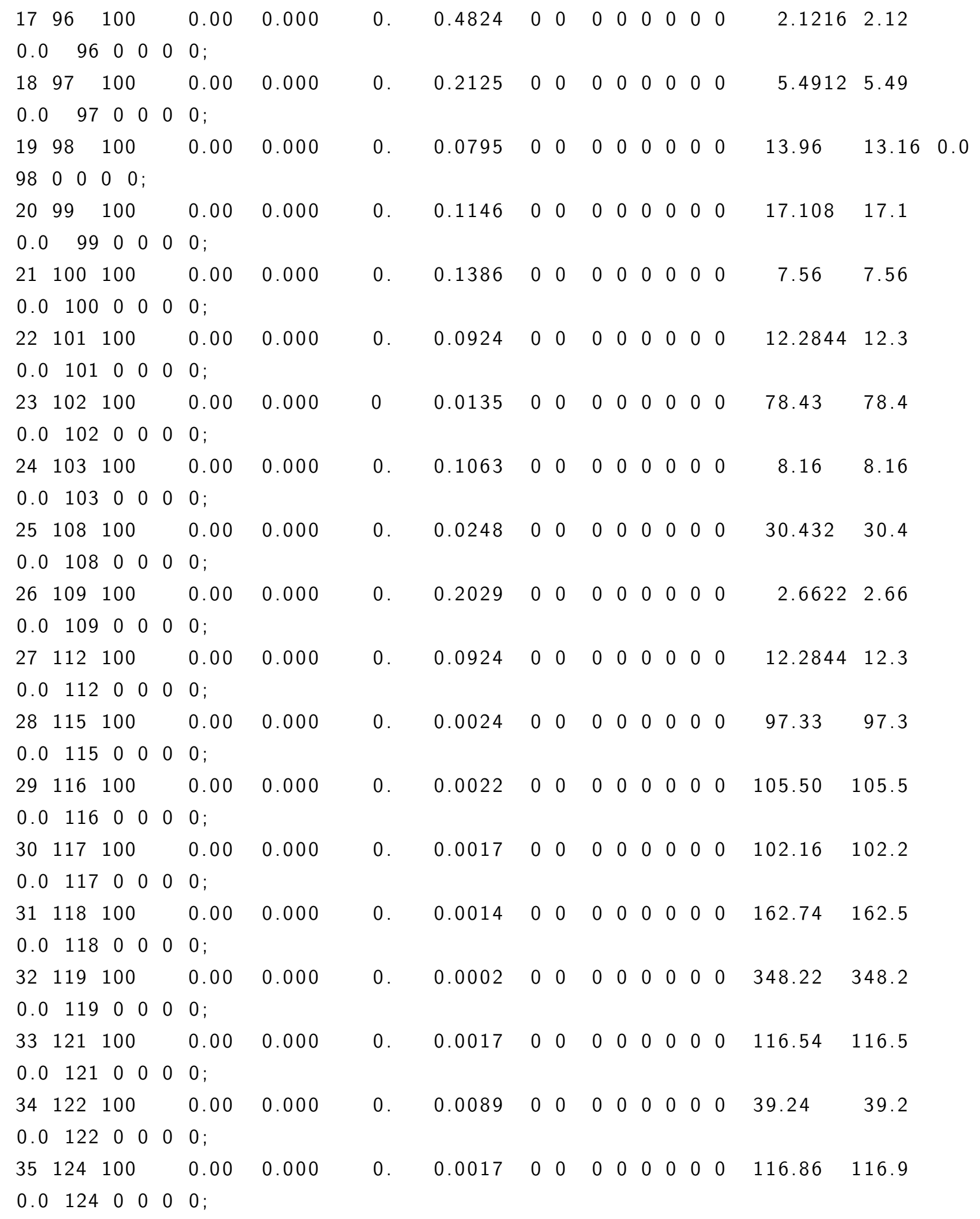




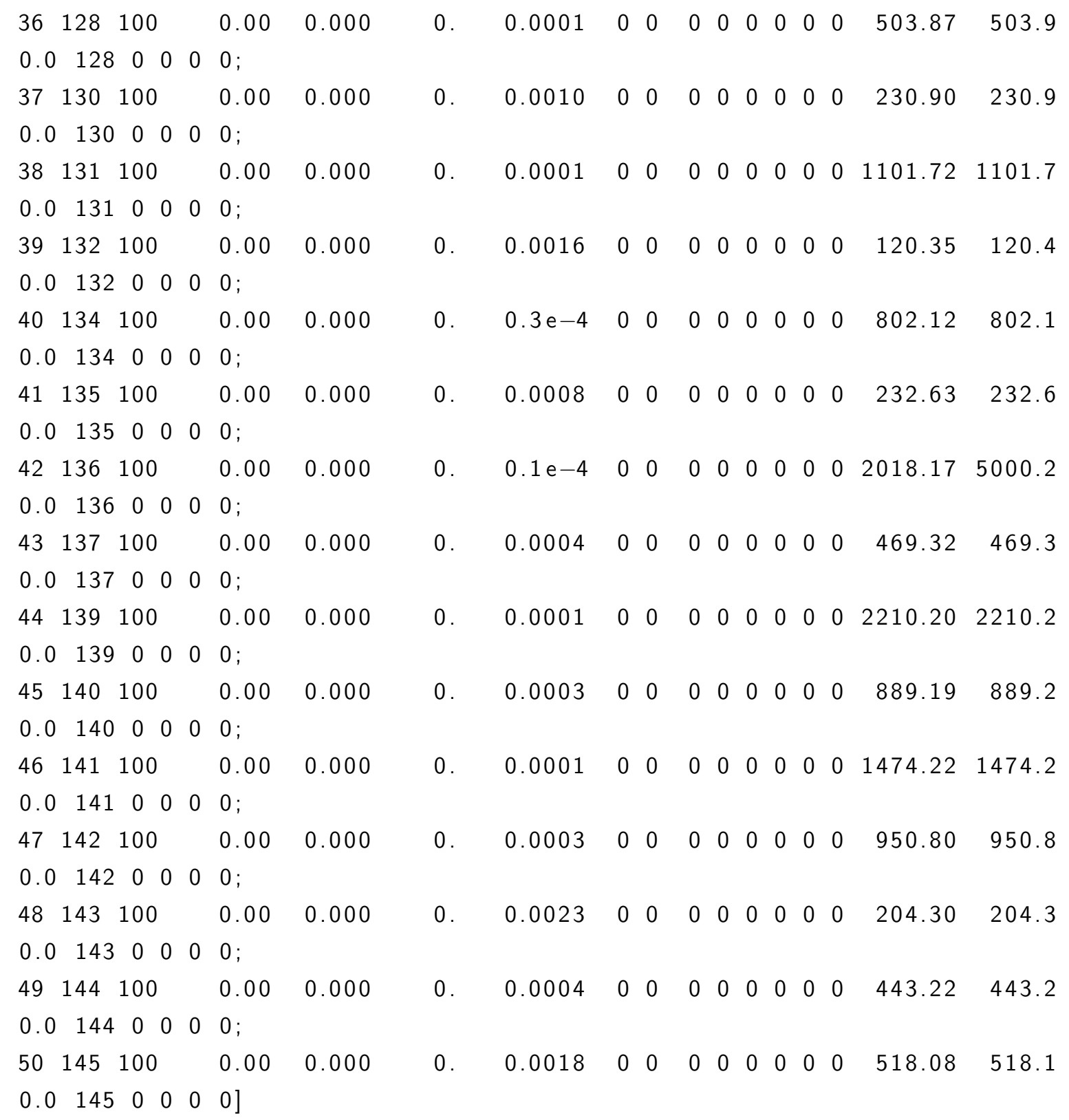




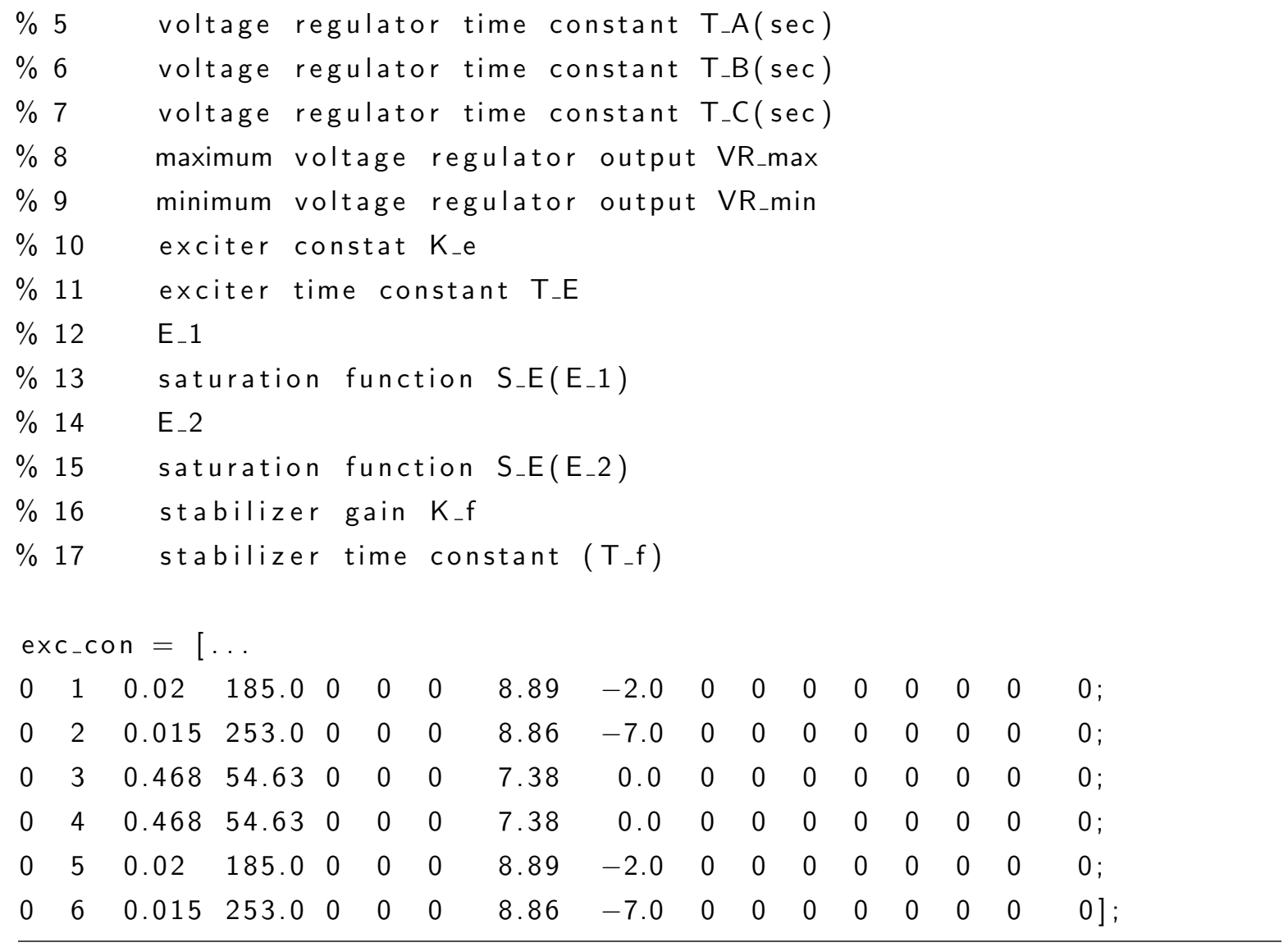


APPENDIX

E

\section{Matlab Code}

\section{E.1 Particle Swarm Optimiztion}

PSO Matlab Function

function [Jss, xss, Jsss, t] = psomax(bounds, evalFN, tmax, c1, c2, n,M)

\% PSOmax run a Partical Swarm Optimization to maximize the objective function.

$\%$ function [x, endPop, bPop, tracelnfo]=ga(bounds, evalFN, tmax, c1, c2, n,M)

$\%$

$\%$ Output Arguments:

$\%$ Jss - the best solution found during the course of the run.

$\%$ xss - the final population.

$\%$ Jsss $\quad-$ a trace of the best population.

$\% \quad \mathrm{t} \quad-$ number of iteration.

$\%$

$\%$ Input Arguments:

$\%$ bounds $\quad-$ a matrix of upper and lower bounds on the variables.

$\%$ evalFN - the name of the evaluation .m function.

$\%$ tmax - maximum number of iteration.

$\% \quad c 1 \quad-$ weighting factor. 


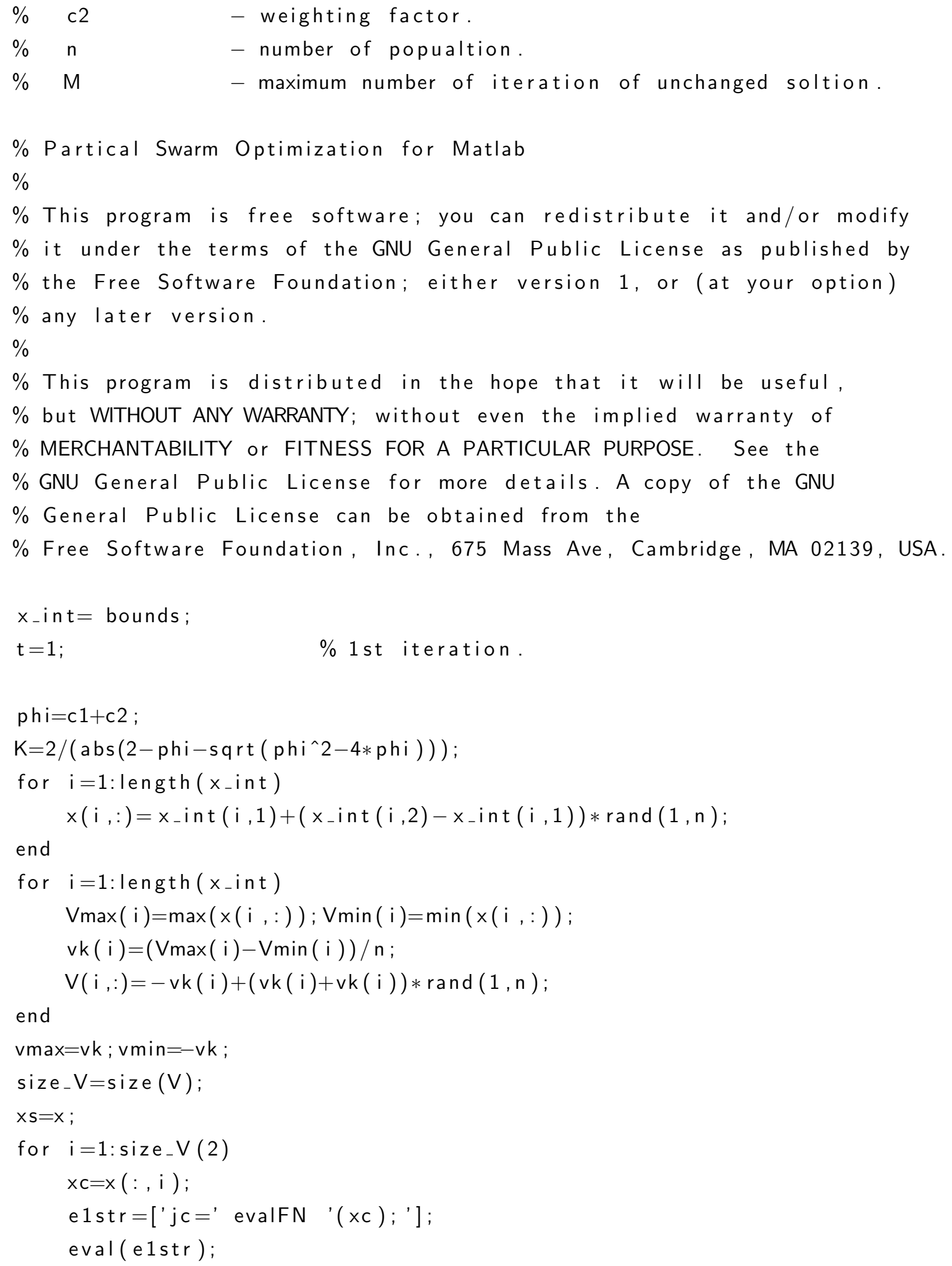




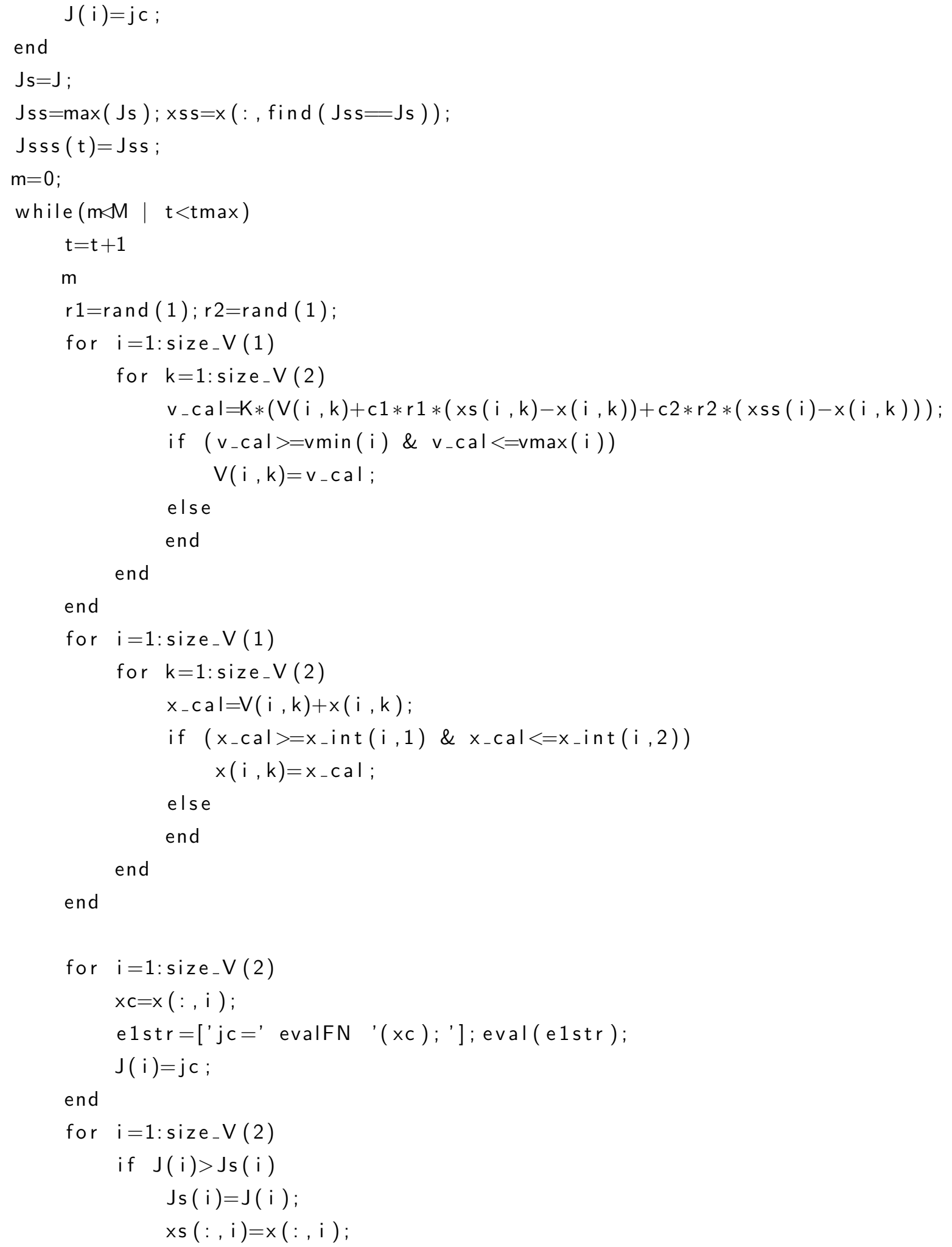




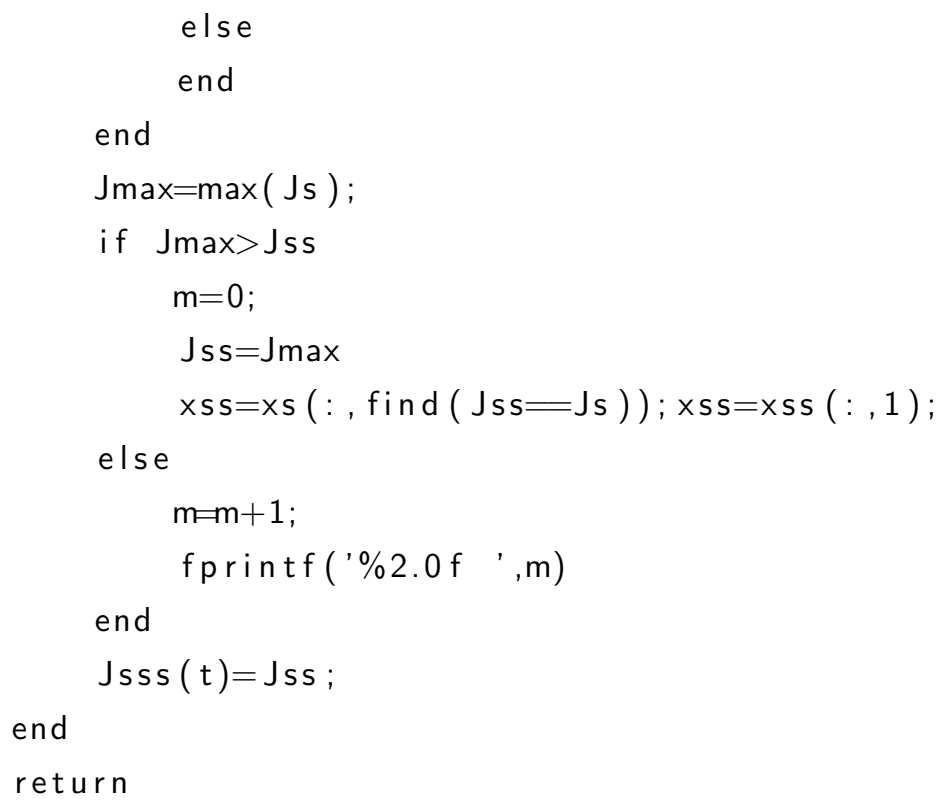




\section{E.2 Reinforcement Learning}

Reinforcement Learning

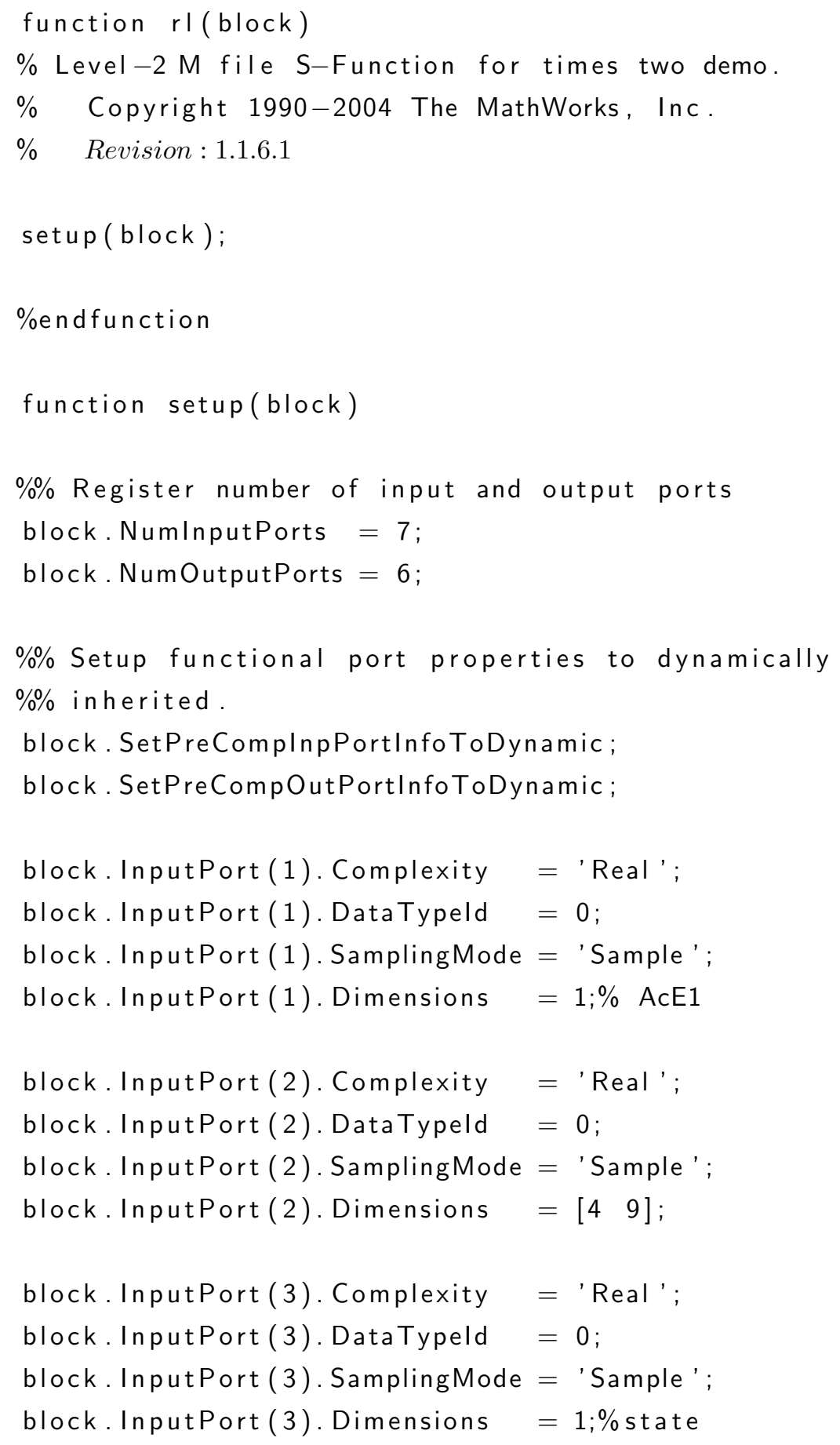




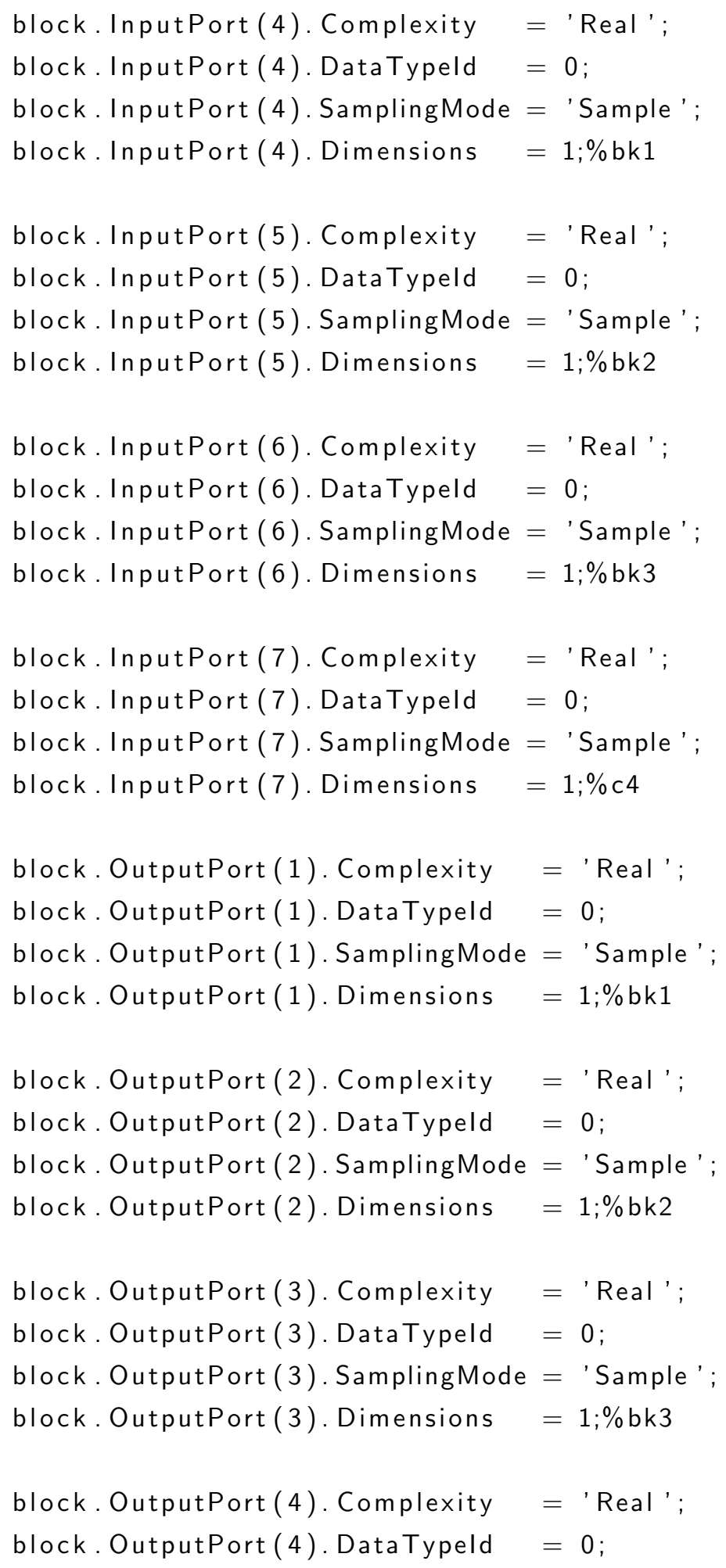


block. OutputPort (4). SamplingMode $=$ 'Sample' $^{\prime}$
block. OutputPort (4). Dimensions $=1 ; \%$ bk 4

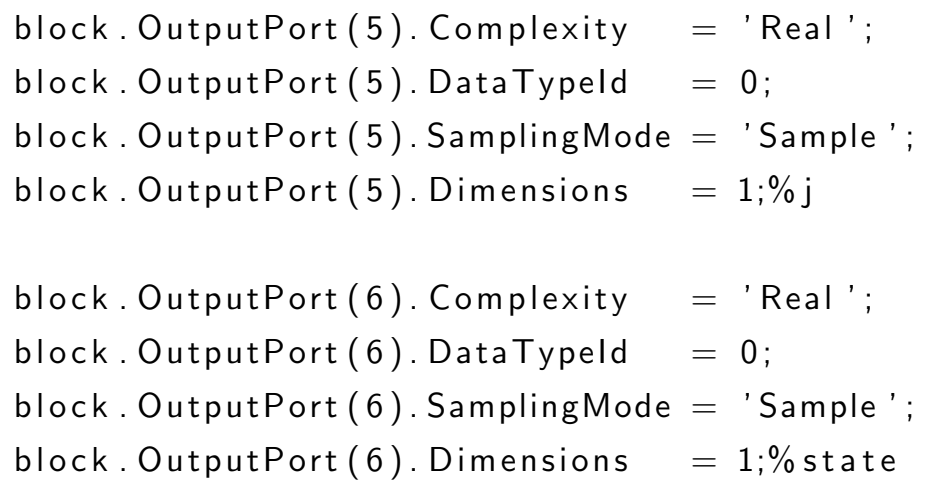




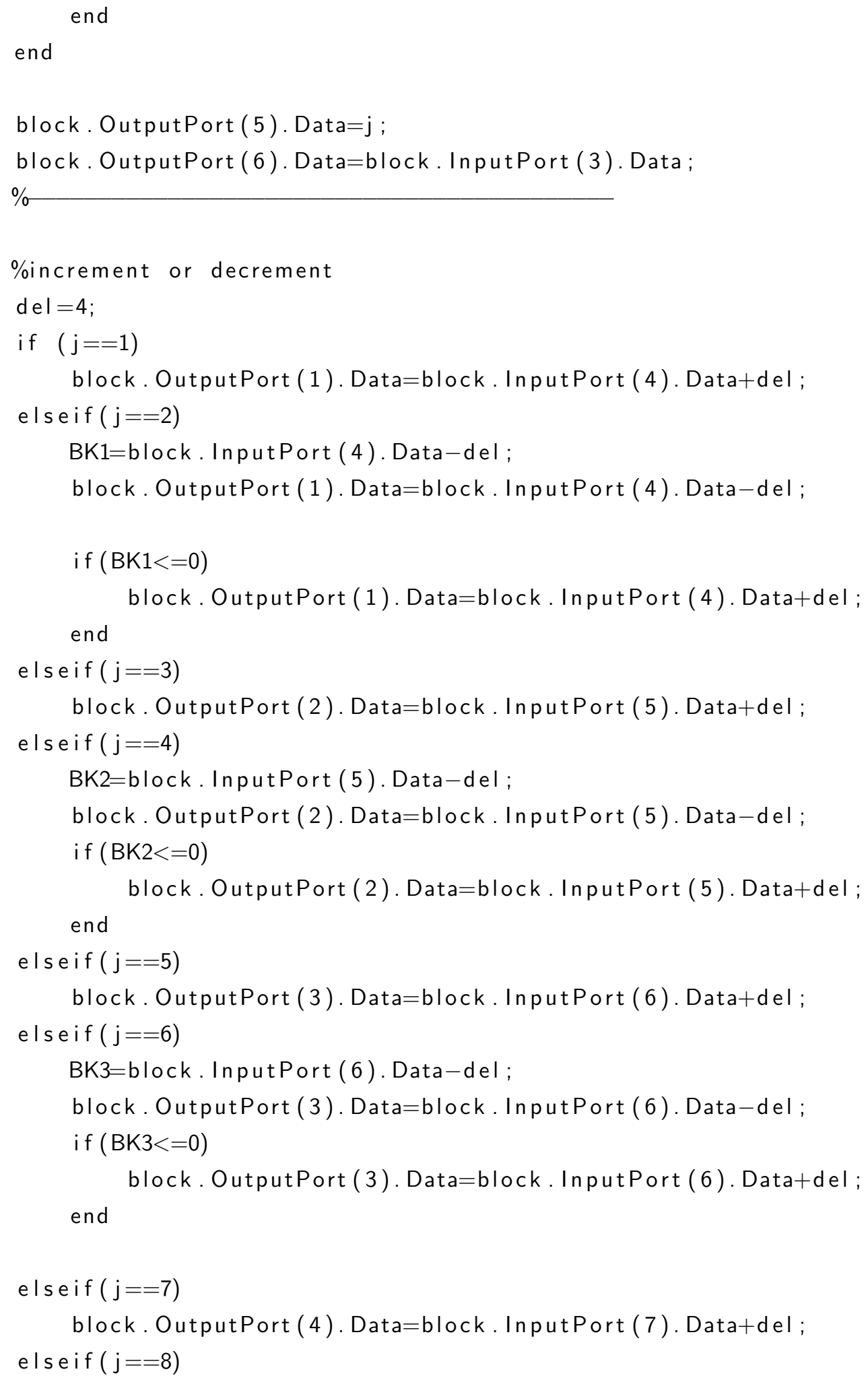




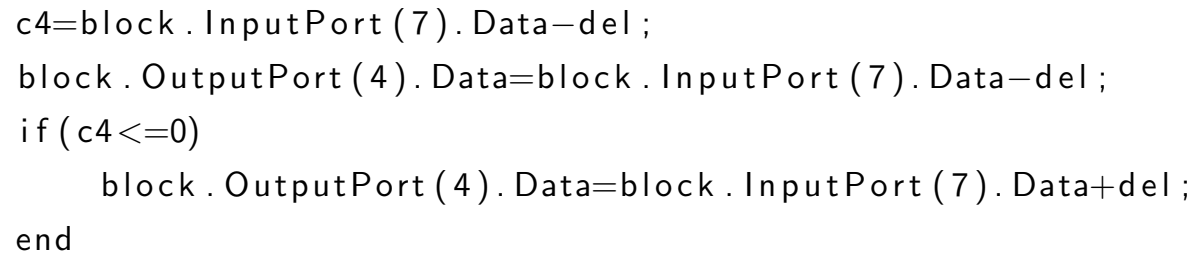

Reinforcement Learning

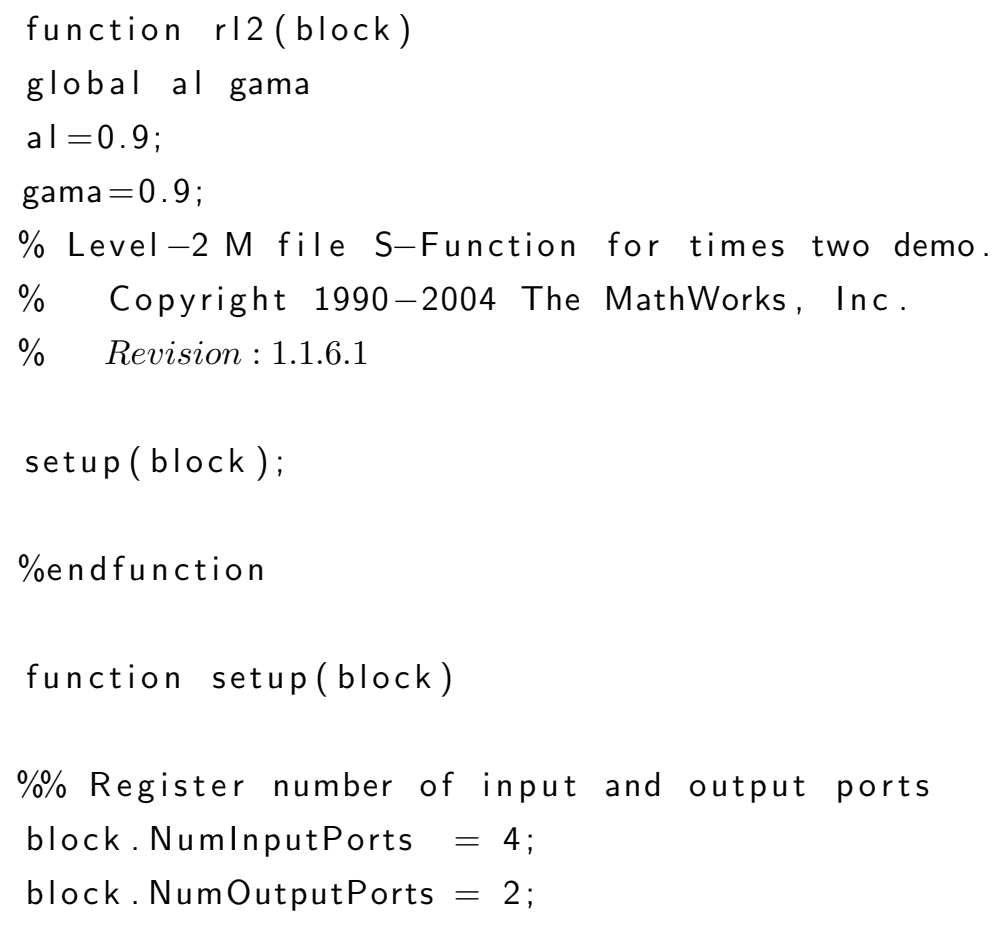




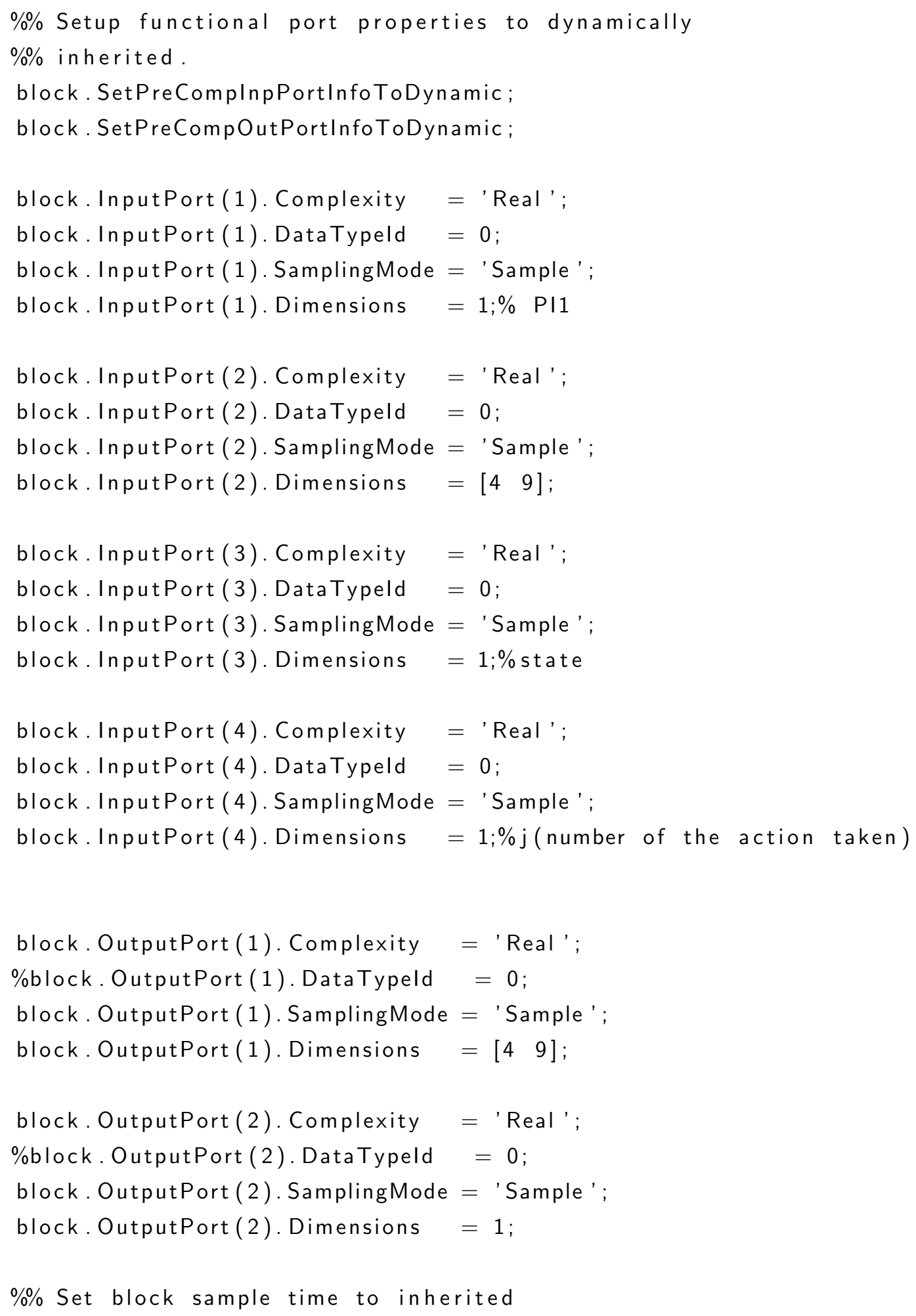




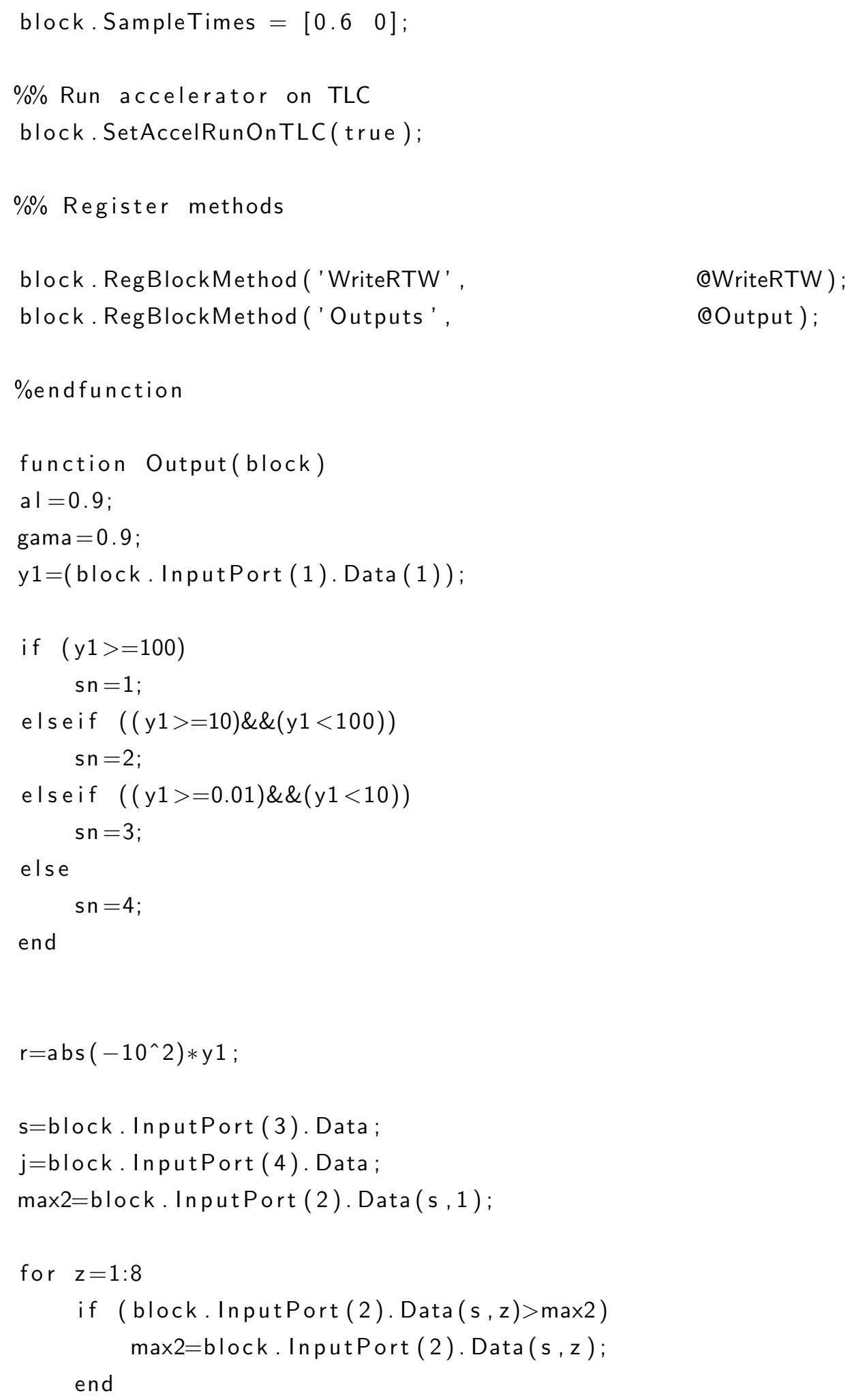


end

block. OutputPort $(1)$. Data $(s, j)=(1-a l) *$ block. InputPort $(2)$. Data $(s, j)+a l *(r+g a m a * m a x 2)$; block. OutputPort (2). Data=sn ;

\%endfunction 


\section{APPENDIX}

$\mathbf{F}$

\section{Appendix-I}

Lemma: Consider $i^{\text {th }}$ subsystem, where $i=1,2, \cdots n$

$$
\begin{aligned}
\dot{x}_{i} & =f_{0 i}\left(x_{i}\right)+g_{0 i}\left(x_{i}\right) z_{1 i} \\
\dot{z}_{1 i} & =f_{1 i}\left(x_{i}, z_{1 i}\right)+g_{1 i}\left(x_{i}, z_{1 i}\right)\left(u_{i}+d_{i}\right)
\end{aligned}
$$

The following control

$$
u_{i}=u_{B i}+u_{D i}
$$

where $u_{B i}, u_{D i}$ are backstepping control and nonlinear damping respectively, guarantees global uniform boundedness of $X=\left(x_{i}, z_{1 i}\right)$ and convergence to the residual set

$$
\Re=\left\{X: \sum_{i=1}^{n}|X| \leq \varsigma_{1}^{-1} \circ \varsigma_{2} \circ \varsigma_{3}^{-1}\left(\sum_{i=1}^{n} \frac{\left\|d_{i}\right\|_{\infty}^{2}}{4 \gamma_{i}}\right)\right\}
$$

where $\varsigma_{1}, \varsigma_{2}$, and $\varsigma_{3}$ are $\kappa_{\infty}$ function such that

$$
\begin{aligned}
\varsigma_{1}(|X|) \leq V(X) & \leq \varsigma_{2}(|X|) \\
\varsigma_{3}(|X|) & \leq W(X)
\end{aligned}
$$


Summation of Lyapunov function is positive definite.

$$
V_{\text {total }}=\sum_{i=1}^{n} V_{1 i}\left(x_{i}, z_{1 i}\right) \quad i=1,2, \cdots n
$$

Obtain derivative of Lyapunov function along closed loop trajectories (F.1), (F.2)

$$
\begin{aligned}
\dot{V}_{\text {total }}=\sum_{i=1}^{n} & \frac{\partial V_{1 i}\left(x_{i}, z_{1 i}\right)}{\partial x_{i}} \dot{x}_{i}+\frac{\partial V_{1 i}\left(x_{i}, z_{1 i}\right)}{\partial z_{1 i}} \dot{z}_{1 i} \quad i=1,2, \cdots n \\
\dot{V}_{\text {total }} & =\sum_{i=1}^{n} \frac{\partial V_{1 i}\left(x_{i}, z_{1 i}\right)}{\partial x_{i}}\left(f_{0 i}\left(x_{i}\right)+g_{0 i}\left(x_{i}\right) z_{1 i}\right)+ \\
& +\frac{\partial V_{1 i}\left(x_{i}, z_{1 i}\right)}{\partial z_{1 i}}\left(f_{1 i}\left(x_{i}, z_{1 i}\right)+g_{1 i}\left(x_{i}, z_{1 i}\right)\left(u_{i}+d_{i}\right)\right)
\end{aligned}
$$

substitute control signal in (F.9)

$$
\begin{aligned}
\dot{V}_{\text {total }} & =\sum_{i=1}^{n} \frac{\partial V_{1 i}\left(x_{i}, z_{1 i}\right)}{\partial x_{i}}\left(f_{0 i}\left(x_{i}\right)+g_{0 i}\left(x_{i}\right) z_{1 i}\right)+ \\
& +\frac{\partial V_{1 i}\left(x_{i}, z_{1 i}\right)}{\partial z_{1 i}}\left(f_{1 i}\left(x_{i}, z_{1 i}\right)+g_{1 i}\left(x_{i}, z_{1 i}\right) \phi_{1 i}\left(x_{i}, z_{i 1}\right)-\right. \\
& \left.-\gamma_{i} \frac{\partial V_{1 i}\left(x_{i}, z_{1 i}\right)}{\partial z_{1 i}} g_{1 i}^{2}\left(x_{i}, z_{1 i}\right)+g_{1 i}\left(x_{i}, z_{1 i}\right) d_{i}\right) \\
\dot{V}_{\text {total }} & =\sum_{i=1}^{n} \frac{\partial V_{1 i}\left(x_{i}, z_{1 i}\right)}{\partial x_{i}}\left(f_{0 i}\left(x_{i}\right)+g_{0 i}\left(x_{i}\right) z_{1 i}\right)+ \\
& +\frac{\partial V_{1 i}\left(x_{i}, z_{1 i}\right)}{\partial z_{1 i}}\left(f_{1 i}\left(x_{i}, z_{1 i}\right)+g_{1 i}\left(x_{i}, z_{1 i}\right) \phi_{1 i}\left(x_{i}, z_{i 1}\right)\right)- \\
& -\gamma_{i}\left(\frac{\partial V_{1 i}\left(x_{i}, z_{1 i}\right)}{\partial z_{1 i}}\right)^{2} g_{1 i}^{2}\left(x_{i}, z_{1 i}\right)+\frac{\partial V_{1 i}\left(x_{i}, z_{1 i}\right)}{\partial z_{1 i}} g_{1 i}\left(x_{i}, z_{1 i}\right) d_{i}
\end{aligned}
$$

use (4.18)

$$
\begin{aligned}
& \sum_{i=1}^{n} \frac{\partial V_{1 i}\left(x_{i}, z_{1 i}\right)}{\partial x_{i}}\left(f_{0 i}\left(x_{i}\right)+g_{0 i}\left(x_{i}\right) z_{1 i}\right)+ \\
+ & \frac{\partial V_{1 i}\left(x_{i}, z_{1 i}\right)}{\partial z_{1 i}}\left(f_{1 i}\left(x_{i}, z_{1 i}\right)+g_{1 i}\left(x_{i}, z_{1 i}\right) \phi_{1 i}\left(x_{i}, z_{i 1}\right)\right) \\
\leq & \sum_{i=1}^{n}-W_{i}\left(x_{i}\right)-k_{1 i}\left(z_{1 i}-\phi_{0 i}\left(x_{i}\right)\right)^{2}
\end{aligned}
$$


substitute (F.12) in (F.11)

$$
\begin{aligned}
\dot{V}_{\text {total }} & \leq \sum_{i=1}^{n}-W_{i}\left(x_{i}\right)-k_{1 i}\left(z_{1 i}-\phi_{0 i}\left(x_{i}\right)\right)^{2}- \\
& -\gamma_{i}\left(\frac{\partial V_{1 i}\left(x_{i}, z_{1 i}\right)}{\partial z_{1 i}}\right)^{2} g_{1 i}^{2}\left(x_{i}, z_{1 i}\right)+\frac{\partial V_{1 i}\left(x_{i}, z_{1 i}\right)}{\partial z_{1 i}} g_{1 i}\left(x_{i}, z_{1 i}\right) d_{i}
\end{aligned}
$$

From Young's inequality know that

$$
A B \leq \gamma A^{2}+\frac{1}{4 \gamma} B^{2} \quad \gamma>0
$$

where $A, B$ are variables, and $\gamma$ is constant. Consider the last term (F.13), and apply (F.14)

$$
\begin{aligned}
\sum_{i=1}^{n} \frac{\partial V_{1 i}\left(x_{i}, z_{1 i}\right)}{\partial z_{1 i}} g_{1 i}\left(x_{i}, z_{1 i}\right) d_{i} & \leq \sum_{i=1}^{n} \gamma\left(\frac{\partial V_{1 i}\left(x_{i}, z_{1 i}\right)}{\partial z_{1 i}} g_{1 i}\left(x_{i}, z_{1 i}\right)\right)^{2}+\frac{1}{4 \gamma_{i}} d_{i}^{2} \\
& \leq \sum_{i=1}^{n} \gamma\left(\frac{\partial V_{1 i}\left(x_{i}, z_{1 i}\right)}{\partial z_{1 i}} g_{1 i}\left(x_{i}, z_{1 i}\right)\right)^{2}+\frac{1}{4 \gamma_{i}}\left\|d_{i}\right\|_{\infty}^{2}
\end{aligned}
$$

Substitute (F.15) in (F.13) and simplify

$$
\dot{V}_{\text {total }} \leq \sum_{i=1}^{n}-W_{i}\left(x_{i}\right)-k_{1 i}\left(z_{1 i}-\phi_{0 i}\left(x_{i}\right)\right)^{2}+\frac{1}{4 \gamma_{i}}\left\|d_{i}\right\|_{\infty}^{2}
$$

From (F.16) it follows that $\dot{V}_{\text {total }}$ is negative definite whenever

$$
\sum_{i=1}^{n} W_{i}\left(x_{i}\right)+k_{1 i}\left(z_{1 i}-\phi_{0 i}\left(x_{i}\right)\right)^{2} \geq \sum_{i=1}^{n} \frac{1}{4 \gamma_{i}}\left\|d_{i}\right\|_{\infty}^{2}
$$

or in simplified form

$$
\sum_{i=1}^{n} W_{i}\left(X_{i}\right) \geq \sum_{i=1}^{n} \frac{1}{4 \gamma_{i}}\left\|d_{i}\right\|_{\infty}^{2}
$$

where $X_{i}=\left(x_{i}, z_{1 i}\right)$. Large $\gamma_{i}$ decreases the effect of disturbance and will guarantee the stability of system. Combining (F.18) with (F.6) results in

$$
\sum_{i=1}^{n}\left|X_{i}\right|>\varsigma_{3}^{-1}\left(\sum_{i=1}^{n} \frac{1}{4 \gamma_{i}}\left\|d_{i}\right\|_{\infty}^{2}\right) \Rightarrow \dot{V}_{\text {total }}<0
$$

Means that if $\sum_{i=1}^{n}\left|X_{i}(0)\right| \leq \varsigma_{3}^{-1}\left(\sum_{i=1}^{n} \frac{1}{4 \gamma_{i}}\left\|d_{i}\right\|_{\infty}^{2}\right)$, then from (F.5)

$$
\sum_{i=1}^{n} V_{i}\left(X_{i}\right) \leq \varsigma_{2} \circ \varsigma_{3}^{-1}\left(\sum_{i=1}^{n} \frac{1}{4 \gamma_{i}}\left\|d_{i}\right\|_{\infty}^{2}\right)
$$


implies that

$$
\sum_{i=1}^{n}\left|X_{i}\right| \leq \varsigma_{1}^{-1} \circ \varsigma_{2} \circ \varsigma_{3}^{-1}\left(\sum_{i=1}^{n} \frac{1}{4 \gamma_{i}}\left\|d_{i}\right\|_{\infty}^{2}\right)
$$

on the other hand if $\sum_{i=1}^{n}\left|X_{i}(0)\right|>\varsigma_{3}^{-1}\left(\sum_{i=1}^{n} \frac{1}{4 \gamma_{i}}\left\|d_{i}\right\|_{\infty}^{2}\right)$, then

$$
\sum_{i=1}^{n} V_{i}\left(X_{i}\right) \leq \sum_{i=1}^{n} V_{i}\left(X_{i}(0)\right)
$$

that implies

$$
\sum_{i=1}^{n}\left|X_{i}\right| \leq \varsigma_{1}^{-1} \circ \varsigma_{2}\left(\sum_{i=1}^{n}\left|X_{i}(0)\right|\right)
$$

Combining (F.21) and (F.23) results in the global uniform boundedness of $X_{i}$. 


\section{APPENDIX}

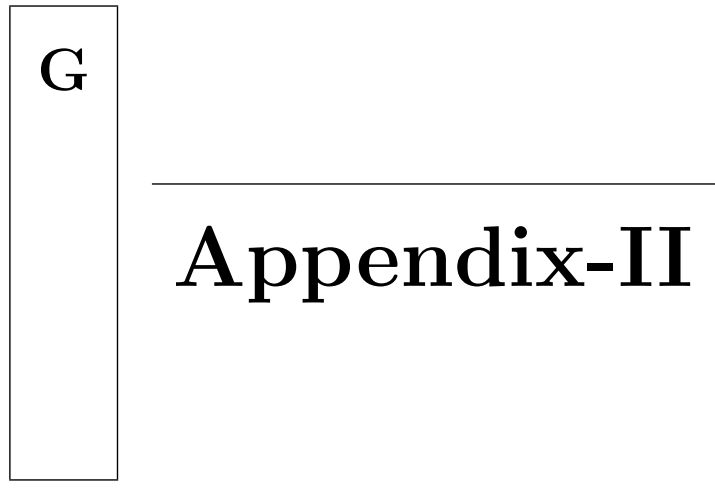

Consider generators (single-axis) connected through a transmission line (Figure G.1). The coupling term for each machine is given in following [35].

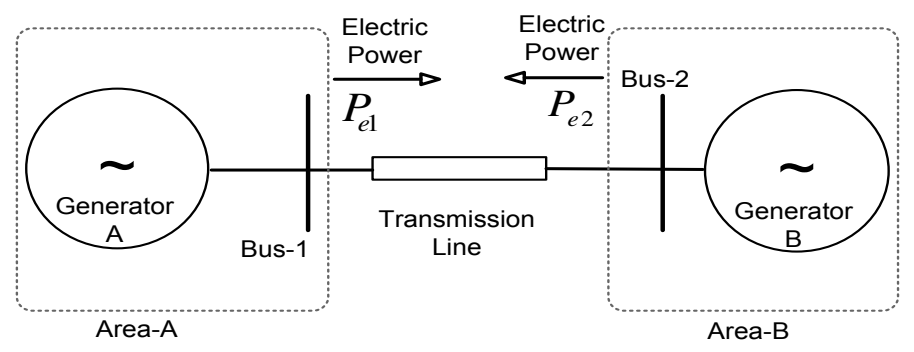

Figure G.1: Schematic view of two generators with single transmission line in between

$$
d_{i}=-\frac{P_{e i}^{\circ}}{T_{q o i}^{\prime}}+\frac{I_{q i} u_{f l d i}^{\circ}}{T_{q o i}^{\prime}}-\frac{I_{d i} I_{q i}\left(x_{d i}-x_{d i}^{\prime}\right)}{T_{q i}^{\prime}}+E_{q i}^{\prime} \dot{I}_{q i}
$$


where algebraic equation are presented as

$$
\begin{aligned}
P_{e i} & =\sum_{j=1}^{n} E_{q i}^{\prime} E_{q j}^{\prime} B_{i j} \sin \left(\delta_{i}-\delta_{j}\right) \\
Q_{e i} & =\sum_{j=1}^{n} E_{q i}^{\prime} E_{q j}^{\prime} B_{i j} \cos \left(\delta_{i}-\delta_{j}\right) \\
E_{d i} & =E_{q i}^{\prime}+\left(x_{d i}-x_{d i}^{\prime}\right) I_{d i} \\
I_{q i} & =\sum_{j=1}^{n} E_{q j}^{\prime} B_{i j} \sin \left(\delta_{i}-\delta_{j}\right) \\
I_{d i} & =-\sum_{j=1}^{n} E_{q j}^{\prime}\left(B_{i j} \cos \left(\delta_{i}-\delta_{j}\right)\right.
\end{aligned}
$$

Assume that $u_{f l d}^{\circ} \approx E$ and use (G.4) to simplify the first three terms of the coupling term that results in $d_{i} \approx E_{q i}^{\prime} \dot{I}_{q i}$. This presents that subsystem dynamics for each generator are nonlinear and coupled through nonlinear coupling equations $I_{q i}(t)$. The main goal is to obtain explicitly the coupling terms $\Phi\left(\Delta P_{t i e}\right)$ as a function of tie line power deviations as mentioned in equation (6.19). Starting point include coupling term that appears in derivative of each Lyapunov function has the following extra term

$$
\dot{V}=-\kappa_{1}\left(\Delta \delta-\alpha_{0}\right)^{2}-\kappa_{2}\left(\Delta \omega-\alpha_{1}\right)^{2}-\kappa_{3}\left(\Delta P_{e}-\alpha_{2}\right)^{2}+\left(\Delta P_{e}-\alpha_{2}\right) d
$$

Here it is shown for simplistic case that the coupling term is a function of tie line power and will counteract any errors in the estimate.

$$
\begin{aligned}
& \Phi\left(\Delta P_{t i e}\right)=\left(\Delta P_{e 1}^{A}-\alpha_{2}^{A}\right) d^{A}+\left(\Delta P_{e 2}^{B}-\alpha_{2}^{B}\right) d^{B} \\
& \Phi\left(\Delta P_{t i e}\right) \approx\left(\Delta P_{e 1}^{A}-\alpha_{2}^{A}\right) E_{q 1}^{\prime} \dot{I}_{q 1}+\left(\Delta P_{e 2}^{B}-\alpha_{2}^{B}\right) E_{q 2}^{\prime} \dot{I}_{q 2}
\end{aligned}
$$

Obtain derivative of (G.5) and substitute in (G.7), then factorizing

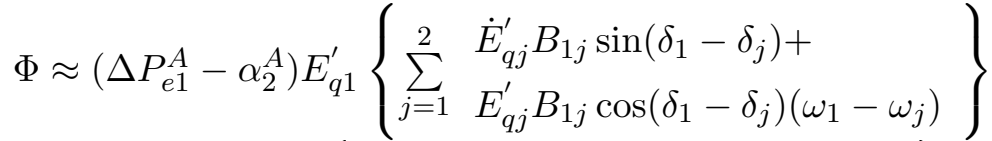

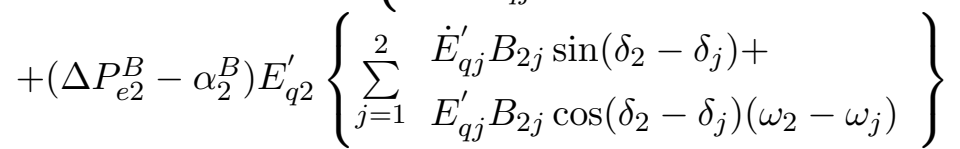


substitute dynamic of quadratic voltage for $j=1,2$

$$
\dot{E}_{q j}^{\prime}=\frac{1}{T_{d o j}^{\prime}}\left(u_{f l d j}-E_{q j}\right)
$$

assume that $u_{f l d j} \approx \sigma E_{q j}$ and $\dot{E}_{q j}^{\prime} \approx \varsigma E_{q j}^{\prime}$ where $\varsigma$ and $\sigma$ are proportional term which are specified through reinforcement learning. Equation (G.8) can be simplified as

$$
\left(\Delta P_{e 1}^{A}-\alpha_{2}^{A}-\Delta P_{e 2}^{B}+\alpha_{2}^{B}\right)\left\{\begin{array}{l}
E_{q 1}^{\prime} E_{q 2}^{\prime} \varsigma B_{12} \sin \left(\delta_{1}-\delta_{2}\right)+ \\
E_{q 1}^{\prime} E_{q 2}^{\prime} \varsigma B_{12} \cos \left(\delta_{1}-\delta_{2}\right)\left(\omega_{1}-\omega_{2}\right)
\end{array}\right\}
$$

Referring to active and reactive power definition (G.2, G.3), equation (G.10) simplify as

$$
\left(\Delta P_{e 2}^{A}-\alpha_{2}^{A}-\Delta P_{e 2}^{B}+\alpha_{2}^{B}\right)\left\{P_{e 1} \varsigma B_{12}-\left(Q_{e 1}-E_{q 1}^{\prime 2} B_{11}\right)\left(\omega_{1}-\omega_{2}\right)\right\}
$$

using (4.48) and (4.38), the first expression becomes

$$
\begin{aligned}
& \Delta P_{e 1}^{A}-\alpha_{2}^{A}-\Delta P_{e 2}^{B}+\alpha_{2}^{B}=\Delta P_{e 1}^{A}-\alpha_{21}^{A} \Delta \delta_{1}- \\
& -\alpha_{22}^{A}\left(\Delta \omega_{1}-\alpha_{1}^{A}\right)-\Delta P_{e 2}^{B}+\alpha_{21}^{B} \Delta \delta_{2}+\alpha_{22}^{B}\left(\Delta \omega_{2}-\alpha_{1}^{B}\right)
\end{aligned}
$$

following assumptions are considered

- $\Delta \omega_{2} \rightarrow \alpha_{1}^{B}, \Delta \omega_{1} \rightarrow \alpha_{1}^{A}$

- $\Delta \delta_{1} \alpha_{21}^{A} \approx \Delta \delta_{2} \alpha_{21}^{B}$

simplify (G.11)

$$
\Phi \approx\left(\Delta P_{e 1}^{A}-\Delta P_{e 2}^{B}\right)\left\{P_{e 1}^{A} \varsigma B_{12}-\left(Q_{e 1}^{B}-E_{q 1}^{\prime 2} B_{11}\right)\left(\omega_{1}-\omega_{2}\right)\right\}
$$


consider the lossless case where active and reactive power for area-1 can be written as $P_{e 1}^{A}=$ $\frac{P_{e 1}^{A}+P_{e 1}^{A}}{2}=\frac{P_{e 1}^{A}-P_{e 2}^{B}}{2}$ similarly for reactive power.

$$
\Phi \approx\left(\Delta P_{e 1}^{A}-\Delta P_{e 2}^{B}\right)\left\{\begin{array}{c}
\frac{\left(\omega_{1}-\omega_{2}\right)}{2}\left(\begin{array}{c}
-Q_{e 1}-Q_{e 2} \\
-E_{q 1}^{\prime 2} B_{11}-E_{q 2}^{\prime 2} B_{22}
\end{array}\right) \\
-\frac{\varsigma}{2}\left(\Delta P_{e 1}^{A}-\Delta P_{e 2}^{B}+P_{e 1}^{\circ A}-P_{e 2}^{\circ B}\right)
\end{array}\right\}
$$

Reactive power expression is written in following form, using (G.3)

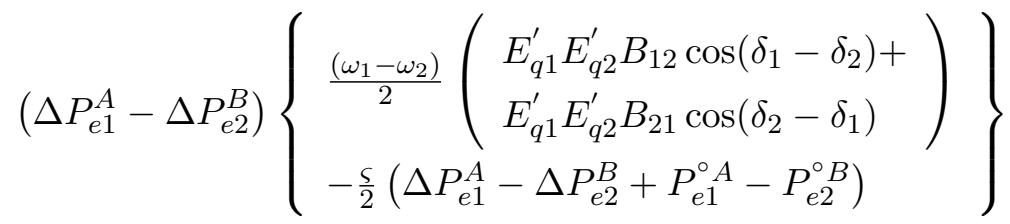

where final expression is obtained

$$
\begin{gathered}
\Phi \approx\left(\Delta P_{t i e}\right)\left\{\frac{1}{2} \frac{\partial \Delta P_{t i e}}{\partial \delta_{12}} \frac{\partial \delta_{12}}{\partial t}-\frac{\varsigma}{2}\left(\Delta P_{t i e}+P_{t i e}^{\circ}\right)\right\} \\
\Phi \approx-\frac{\varsigma}{2} \Delta P_{t i e}^{2}-\frac{\varsigma}{2} P_{t i e}^{\circ} \Delta P_{t i e}+\frac{1}{2} \frac{\partial \Delta P_{t i e}}{\partial \delta_{12}} \frac{\partial \delta_{12}}{\partial t}
\end{gathered}
$$

where $\Delta P_{\text {tie }}=\Delta P_{e 1}^{A}-\Delta P_{e 2}^{B}$. Therefore the coupling term is approximated as a tie-line power from area 1 to 2 . 


\section{References}

[1] Y. L. Abdel-Magid, M. A. Abido, and A. H. Mantaway, "Robust Tuning of Power System Stabilizers in Multimachine Power Systems," IEEE Transaction on Power Systems, Vol. 15, No. 2, pp. 735-740, 2000.

[2] Y. L. Abdel-Magid, M. A. Abido, S. Al-Baiyat, and A. H. Mantaway, "Simultaneous Stabilization of Multimachine Power Systems via Genetic Algorithms," IEEE Transaction on Power Systems, Vol. 14, No. 4, pp. 1428-1439, 1999.

[3] M. A. Abido, "Optimal Design of Power-System Stabilizers Using Particle Swarm Optimization," IEEE Transactions on Energy Conversion, Vol. 17, No.3, September 2002.

[4] M. A. Abido, "Particle swarm optimization for multi machine power system stabilizer design," IEEE Power Engineering Society Summer Meeting, Vol. 3, July 2001.

[5] M. A. Abido, Y. L. Abdel-Magid "Robust design of multimachine power system stabilizers using tabu search algorithm," IEE Proceedings Generation, Transmission and Distribution, Vol. 147, pp. 387-394, November 2000. 
[6] M. R. Alrashidi, M. E. El-Hawary, "A Survey of Particle Swarm Optimization Application in Electric Power Systems," Journal of Electric Power Components and systems, Vol. 34, 2006, pp. 1349-1357.

[7] A. P. Alves da Silva, P. J. Abrão, "Applications of Evolutionary Computation in Electric Power Systems," Proceedings of the 2002 Congress on Evolutionary Computation CEC, Vol. 2, pp. 1057-1062, 2002.

[8] M. Amin, "Powering the $21^{\text {st }}$ Century," IEEE Power $\&$ Energy, pp. 93-96, March-April, 2005.

[9] Anderson, P. M., Fouad, A. A., Power System Control and Stability, IEEE Press, 1994.

[10] G. Bartolini, A. Ferrara, E. Usai "Chattering Avoidance by second Order Sliding Mode Control," IEEE Transactions on Automatic Control, Vol. 43, No. 2, pp. 241-246, 1998.

[11] A. S. Bazanella, P. V. Kokotović, A. S. e Silva "A Dynamic Extension of $L_{g} V$ Controllers," Proceedings of the 36th Conference on Decision $\&$ Control, San diego, California, December 1997.

[12] G. E. Boukarim, S. Wang, J. H. Chow, G. N. Taranto, N. Martins "A Comparison of Classical, Robust, and Decentralized Control Designs for Multiple Power System Stabilizers," IEEE Transactions on Power Systems, Vol. 15, No. 4, November 2000.

[13] D. S. Brereton, D. G. Lewis, C. C. Young, "representation of Induction Motor Loads During Power System Stability Studies," AIEE Transactions Vol. 76, Part-III, pp. 451460, 1957.

[14] L. Cai, I. Erlich, "Simultaneous Coordinated Tuning of PSS and FACTS Damping Controllers in Large Power Systems," IEEE Transactions on Power Systems, Vol. 20, No. 1, Feb 2005.

[15] J. W. Chapman, M. D. Ilić, "Some Robustness Results for Feedback Linearizing Control of Generator Excitation," Proceedings of 31st Conference on Decision \& Control, Tuscon, Arizona, December 1992. 
[16] J. W. Chapman, M. D. Ilic, C. A. King, "Stabilizing a Multi-machine Power System via Decentralized Feedback Linearizing Excitation Control," IEEE Transactions on Power Systems, Vol. 8, No.3, August 1993.

[17] , B. Chaudhuri, R. Majumder, B. C. Pal, "Wide-Area Measurement-Based Stabilizing Control of Power System Considering Signal Transmission Delay," IEEE transactions on Power Systems, Vol. 19, no. 4, November 2004.

[18] M. A. Choudhry, K. Lateef, "Robust Design of Dynamic Compensator for HV AC Power System," Proc. 22nd North American Power Symposium, October, pp. 2-7, 1990.

[19] M. Clerc, J. Kennedy, "The particle Swarm - Explosion, Stability, and Convergence in Multidimensional Complex Space," IEEE Transactions on Evolutionary Computations, Vol. 6, No. 1, pp. 58-73, 2002.

[20] J. H. Chow, L. P. Harris, M. A. Kale, H. A. Othman, J. J. Sanchez-Gasca, G. E. Terwilliger, " Robust control design of power system stabilizers using multivariable frequency domain techniques," Proceedings of the 29th IEEE Conference on Decision and Control, Honolulu, HI, USA, 1990.

[21] S. J. Cheng, Y. S. Chow, O. P. Malik, G. S. Hope, ” An Adaptive Synchronous Machine Stabilizer," IEEE Transactions on Power Systems, Vol. 1, No. 3, pp. 101-109, 1986.

[22] T. K. Das, S. R. S. Jeti, G. K. Venayagamoorthy, "Optimal Design of SVC Damping Controllers with Wide Area Measurements Using Small Population Based PSO," International Joint Conference of Neural Networks, July 16-21, Vancouver, BC, Canada, 2006.

[23] F. P. de Mello and C. Concordia, "Concepts of Synchronous Machine Stability as Affected by Excitation Control," IEEE Transaction on Power System, Vol. PAS-88(4), pp. 316-329, 1969.

[24] Y. Del Valle, G. K. Venayagamoorthy, S. Mohagheghi, J. C. Hernandez, R. G. Harley, "Particle Swarm Optimization: Basic Concepts, Variants and Applications in Power Systems," IEEE Transaction on Evolutionary Computation, Februuary 2007. 
[25] A. L. B. do Bomfim and G. N. Taranto and D. M. Falcão, "Simultaneous Tuning of Power System Damping Controllers Using Genetic Algorithms," IEEE Transaction on Power System, Vol. 15, pp. 163-169, 2000.

[26] D. Ernst, M. Glavić, L. Wehenkel"Power System Stability Control: Reinforcement Learning Framework," IEEE Transactions on Power Systems, Vol. 19, No. 1, 2004.

[27] P. Fairley, "The Unruly Power Grid," IEEE Spectrum, August, pp. 22-25, 2004.

[28] L. Fan, A. Feliachi, "Decentralized Stabilization of Nonlinear Electric Power Systems Using Local Measurements and Feedback Linearization," Proceeding 43rd IEEE Midwest Symposium on Circuits and Systems, Lansing MI, August 2000.

[29] B. Fardanesh, "Future Trends in Power System Control," IEEE Computer Applications in Power, July, pp. 24-31, 2002.

[30] A. Feliachi, "On the Control of Re-Structured Electric Power systems," International Journal of Control, Automation, and Systems, Special Issue on Recent Advances in Power System Control, Vol. 3, No. 2, pp. 363-375, 2005.

[31] A. Fouad, V. Vittal, Power System Transient Stability Analysis Using Transient Energy Function Method, Kluwer Academic Publishers, 1989.

[32] R. A. Freeman, P. V. Kokotović, "Tools and Procedure for Robust Control of Nonlinear Systems" Proceedings of 33rd Conference on Decision 83 Control, Lake Buena Vista, Florida, December, 1994.

[33] M/ Glavić, D. Ernst, L. Wehenkel, "Combining a Stability and Performance-Oriented Control in Power Systems," IEEE Transaction on Power Systems, Vol. 2, No. 1, February 2005 .

[34] M/ Glavić, D. Ernst, L. Wehenkel, "Damping Control by Fusion of Reinforcement Learning and Control Lyapunov Functions," 38th North American Power Symposium, NAPS, pp. 361-367, September 2006.

[35] Y. Guo, D. J. Hill, Y. Wang, "Nonlinear Decentralized Control of Large-scale Power Systems," IEEE Transaction on Automatica, Vol. 36, pp. 1275-1289, 2000. 
[36] A. Hasanović, A. Feliachi, "Robust PSS Tuning Through Multiobjective Optimization," PSCE, IEEE Power Systems and Exposition, New York, October 2004.

[37] A. Hasanović, A. Feliachi, "Genetic algorithm based inter-area oscillation damping Controller Design Using MATLAB," PES, Power Engineering Society Summer Meeting, Vol. 3, pp. 1136 - 1141, 2002.

[38] A. E. Hammad, "Analysis of Power System Stability Enhancement by Static Var Compensator," IEEE Trans. On Power Systems, Vol. 1, No. 4, Nov. 1986.

[39] Hingorani, N. G., "Flexible AC Transmission," IEEE Spectrum, pp. 40-44, April 1993.

[40] IEEE Working Group-H7 of The Relaying Channels, Subcommittee of the IEEE Power System Relaying Committee, "Synchronized Sampling and Phasor Measurements for Relaying and Control," IEEE Transactions on Power System Delivery, Vol. 9, No. 1, January 1994.

[41] IEEE/CIGRE Joint Task Force on Stability Terms and Definitions, "Definition and Classification of Power System stability," IEEE Transactions on Power Systems, Vol. 19, No. 2, pp. 1387-1401, 2004.

[42] IEEE Recommended Practice for Excitation System Models for Power System Stability Studies, Energy Development and Power Generating Committee of the Power Engineering Society, 19 March 1992.

[43] A. Isidori, Nonlinear Control Systems, Springer-Verlag, New York, 1989.

[44] M. D. Ilić, H. Allen, W. Chapman, C. A. King, J. H. Lang, E. Litvinov, "Preventing future blackouts by means of enhanced electric power systems control: from complexity to order," Proceedings of the IEEE, Vol. 93, No. 11, November 2005.

[45] M. D. Ilić, "From Hierarchical to Open Access Electric Power Systems," Proceedings of IEEE, Vol. 95, No. 5, May 2007.

[46] S. Jain, F. Khorrami, B. Fardanesh, "Adaptive Nonlinear Excitation Control of Power Systems with Unknown Interconnections," Proceeding of the 40th IEEE Conference on Decision and Control, Orlando, Florida USA, December, 2001. IEEE Transaction on Control Systems Technology, Vol. 2, No. 4, December 1994. 
[47] S. Jain, F. Khorrami, "Decentralized Adaptive Output Feedback Control of Large scale Interconnected Nonlinear System," Proceedings of the American Control Conference, Seattle, Washington, June, 1995.

[48] I. Kamwa, R. Gordin, G. Trudel, "IEEE PSS2B Versus PSS4B: The Limits of Performance on Modern Power System Stabilizers," IEEE Transactions on Power Systems, Vol. 20, No. 2, May 2005.

[49] I. Kanellakopoulos, "Robustification Tools for Nonlinear Control Design," Proceedings of the 33rd Conference on Decision \& Control, Lake Buena Vista, FL, December 1994.

[50] I. Kanellakopoulos, P. V. Kokotović, A. S. Morse "A Toolkit for Nonlinear Feedback Design," System \& Control Letters, Vol. 18, pp. 83-92, 1992.

[51] A. Karimi, K. Schoder, A. Feliachi, "Decentralized Backstepping Control of Power Systems with Observation Decoupled State Space Method," PSCE, IEEE Power Systems and Exposition, New York, October, 2004.

[52] A. Karimi, A. Al-Hinai, K. Schoder, A. Feliachi, "Power System Stability Enhancement Using Backstepping Controller Tuned by Particle Swarm Optimization Technique," PES, IEEE Power Engineering Society, San Francisco, California, June, 2005.

[53] A. Karimi, A. Feliachi, "Decentralized Extended-Backstepping Control of Power Systems," PES, IEEE Power Engineering Society, Montreal, Canada, 18-22 June, 2006.

[54] A. Karimi, A. Feliachi, "PSO-tuned Adaptive Backstepping control of Power Systems," PSCE, IEEE Power Systems Conference and Exposition, Atlanta, Georgia, October 29November 1, 2006.

[55] A. Karimi, A. Feliachi, "Decentralized adaptive backstepping control of electric power systems," Electric Power Systems Research, Volume 78, Issue 3, pp. 484-493, March 2008.

[56] A. Karimi, A. Feliachi, M. A. Choudhry, "On an SVC Backstepping Damping Nonlinear Controller Design for Power System," Bulk Power System Dynamics and Control-VII, Charlston, South Carolina, USA, August 19-24, 2007. 
[57] A. Karimi, M. A. Choudhry, A. Feliachi "Coordinated Backstepping Controls for Power System Stability Enhancement," North American Power Symposium, Calgary, Canada, Sept 28 th to 30 th, 2008.

[58] D. Karlsson, M. Hemmingsston, S. Lindhal, "Wide Area System Monitoring and Control," IEEE Power and Energy Magazine, pp. 68-76, September-October, 2004.

[59] J. Kennedy, R. Eberhart, "Particle Swarm Optimization," Proceeding of IEEE international conference on Neural Networks (ICNN'95), Perth, Australia, Vol. IV, pp. 19421948, 1995.

[60] H. K. Khalil, Nonlinear Systems, Macmillan Publishing Company, 1992.

[61] M. Klein, G. J. Rogers, P. Kundur, "A fundamental study of inter-area oscillations in power systems", IEEE Transactions on Power Systems, Vol. 6, Issue 3, pp. 914 - 921, August 1991 .

[62] P. V. Kokotović, "The Joy of Feedback: Nonlinear and Adaptive," IEEE Control Systems Magazine, Vol. 12, pp. 7-17, 1992.

[63] P. V. Kokotovic, R. A. Freeman, "Tools and Procedures for Robust Control of Nonlinear Systems," Proceedings of the 33rd Conference on Decision and Control, Lake Buena Vista, FL, December 1994.

[64] M. Krstić, I. Kanellakopoulos, P. Kokotović, Nonlinear and Adaptive Control Design, John Wiley \& Sons, Inc. 1995.

[65] P. Kundur, Power System Stability and Control, McGraw-Hill, 1994.

[66] P. Kundur, "Effective Use of Power System Stabilizers for Enhancement of Power System Reliability," PES, IEEE Power Engineering Society, Edmonton, Alta., Canada, pp. 96103, July 1999.

[67] K. Y. Lee, M. A. El-Sharkawi, "Modern Heuristic Optimization Optimization Techniques: Theory and Applications to Power Systems," Wiley-Interscience, 2008.

[68] X. Lei, E. N. Lerch, and D. Povh, "Optimization and Coordination of Damping Controls for Improving System Dynamic Performance," IEEE Transactions on Power Systems, Vol. 16, No. 3, pp. 473-480, August 2001. 
[69] E. N. Lerch, D. Povh, and L. Xu, "Advanced SVC Control for Damping Power System Oscillations," IEEE Transactions on Power Systems, Vol. 2 (6), pp. 524-535, May 1991.

[70] J. Machowski, J. W. Bialek, S. Robak, and J. R. Bumby, "Excitation Control System for Use with Synchronous Generators," IEE Proceedings - Generation, Transmission and Distribution, Vol. 145, Issue 5, pp. 537-546, 1998.

[71] S. Moscolo, G. Grassi, "Controlling Chaotic Systems with Disturbance using Backstepping Design and Nonlinear Damping," COC, CONTROL of OSCILLATIONS and CHAOS, St. Petersburg, Russia, 2000.

[72] F. Okou., L. A. Dessaint, O. Akhrif, "Power Systems Stability Enhancement Using a Wide-Area Signals Based Hierarchical Controller", IEEE Transactions on Power Systems, Vol. 2, No. 3, pp. 1465-1477, August 2005.

[73] F. Okou., O. Akhrif, L. A. Dessaint, "Novel Modeling Approach for Decentralized Voltage and Speed Control of Multi-machine Power Systems", International Journal of Control, Vol. 76, pp. 845-857, May 2003.

[74] M. A. Pai, Energy Function Analysis for Power System Stability, Kluwer Academic Publishers, 1989.

[75] P. C. Parks, "A. M. Lyapunov's Stability Theory-100 Years on", IMA Journal of Mathematical Control and Information, Vol. 9, pp. 275-303, 1992.

[76] P. Pourbiek, M. J. Gibbard, "Damping and Synchronizing Torque Induced on Generators by FACTS Stabilizers in Multimachine Power Systems," IEEE Transactions on Power Systems, Vol. 11, pp. 1920-1925, November 1996.

[77] P. Pourbiek, M. J. Gibbard, "Simultaneous coordination of Power System Stabilizers and FACTS device stabilizers in a multimachine power system for enhancing dynamic performance," IEEE Transactions on Power Systems, Vol. 13, no. 2, pp. 473-479, 1998.

[78] G. Rogers, Power System Oscillations, Kluwer Academic Publishers, 2000.

[79] A. R. Roosta, D. Georges, N. Hadj-Said, "Nonlinear Control for Power Systems based on a Backstepping Method," Proceeding of the 40th IEEE Conference on Decision and Control, Orlando, Florida USA, December, 2001. 
[80] J. J. Sanchez-Gasca, "Coordinated control of two FACTS devices for damping inter-area oscillations," IEEE PES summer meeting, Berlin, Germany, July 1997.

[81] P. W. Sauer, M. A. Pai, Power System Dynamics and Stability, Prentice-Hall, 1998.

[82] K. Schoder, A. Hasanović, A. Feliachi, and A. Hasanović, "PAT: a power analysis toolbox for MATLAB /Simulink," IEEE Transaction on Power System, Vol. 18, No. 1, pp. 42-47, February 2003.

[83] A. F. Snyder, "Delay input wide area stability control with synchronized phasore measurements," IEEE PES summer meeting, Vol. 2, pp. 1009-1014, 2000.

[84] E. D. Sontag, "A Lyapunov-like characterization of asymptotic controllability," SIAM Journal of Control and Optimization, Vol. 21, pp. 462-471, 1983.

[85] R. S. Sutton, A. G. Barto, Reinforcement Learning: An Introduction (Adaptive Computation and Machine Learning), MIT press, March 1998.

[86] V. I. Utkin, "Variable Structure Control Systems with Sliding Modes," IEEE Transaction on Automatic Control, Vol. AC-22, No.2, pp. 210-222, 1977.

[87] Y. Wang, H. Chen, R. Zhou, D. J. Hill, "Studies of Voltage Stability via a Nonlinear SVc Control," IEEE Power Engineering Society Winter Meeting, Singapore, Vol. 2, pp. 1348-1353, January 2000.

[88] Y. Wang, L. Xie, D. J. Hill, "Robust Nonlinear Controller Design for Transient Stability Enhancement of Power Systems," Proceedings of 31st Conference on Decision 83 Control, Tuscon, Arizona, December 1992.

[89] Y. Wang, G. Guo, D. J. Hill and L. Gao, "Nonlinear Decentrilized Control For Multimachine Power System Transient Stability Enhancement," Proceedings of Stockholm Power Tech International Symposium on Electric Power Engineering, Stockholm, Sweden, pp.435-440, 1995.

[90] X. G. Yontag, S. K. Spurgeon, C. Edwards, "Decentralized Sliding Mode Control for Multimachine Power Systems Using Only Output Information," The 29th Annual Conference of the IEEE on Industrial Electronics Society, IECON, Vol. 2, pp. 1944-1949, 2003. 
[91] X. Yang, A. Feliachi, "Stabilization of Inter-area Oscillation Modes through Excitation Systems," IEEE Transactions on Power Apparatus and Systems Vol. 9, No.1, pp. 494502, February, 1994.

[92] J. Zaborszky, K. W. Whang, K. V. Prasad, "Stabilizing Control in Emergencies PART I," IEEE Transactions on Power Apparatus and Systems Vol. PAS-100, No.5, May, 1981.

[93] Y. Zhang, C. Wen, Y. C. Soh, "Transient Stability Enhancement of Power Systems By Robust Adaptive Control with Saturation Constraint," Proceedings of IEEE International Conference on Control Applications, Kohala Coast-Island of Hawaii, USA, August 1999.

[94] Q. Zhao, J. Jiang, "Robust SVC Controller Design for Improving Power System Damping," IEEE Transactions on Energy Conversion, Vol. 10, pp. 201-209, June 1995.

[95] C. Zhu, R. Zhou, Y. Wang, "A New Decentrilized Nonlinear Voltage Controller For Multimachine Power Systems," IEEE Transactions on Power Systems, Vol. 13, pp. 211216, February 1998.

[96] P. Kundur, M. Klein, G. J. Rogers, M. S. Zywno, "Application of Power System Stabilizers for Enhancement of Overall System Stability," IEEE Transactions on Power Systems, Vol. 4, No. 2, May 1989. 